\title{
Electrochemical synthesis of fluorinated ketones from enol acetates and sodium perfluoroalkyl sulfinates
}

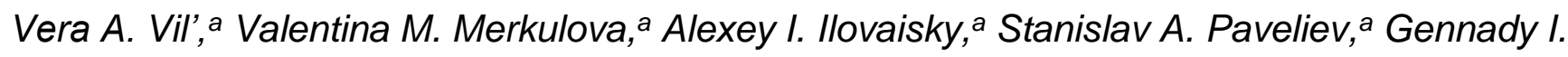
Nikishin a and Alexander O. Terent'ev *a

${ }^{a}$ N. D. Zelinsky Institute of Organic Chemistry, Russian Academy of Sciences, 47 Leninsky Prospekt, 119991 Moscow, Russian Federation

alterex@yandex.ru

General materials and methods S2

General Procedure for Cyclic Voltammetry S2

Enol acetates data. S2

Experimental procedures for Table 1 S6

Electrolysis of silyl enol ether with $\mathrm{CF}_{3} \mathrm{SO}_{2} \mathrm{Na}$....... S7

General Experimental Procedure for Scheme 2. . 77

General Experimental Procedure for Scheme 3. $\mathrm{S} 12$

Experimental procedure for Scheme 4a. S14

Experimental procedure for Scheme 4b. S15

Experimental procedure for Scheme 4c. S15

Experimental procedure for Scheme 4d. S15

$\mathrm{CV}$ data of TEMPO and $\mathrm{CF}_{3} \mathrm{SO}_{2} \mathrm{Na}$ S16

$\mathrm{CV}$ data of deaerated and aerated mixtures of $\mathrm{CF}_{3} \mathrm{SO}_{2} \mathrm{Na}$ and enol acetate $\mathrm{S} 17$

References $\mathrm{S} 18$

NMR spectra of starting enol acetates S20

NMR spectra of obtained fluorinated ketones S50

HRMS spectra of obtained fluorinated ketones. 


\section{General materials and methods}

${ }^{1} \mathrm{H}$ and ${ }^{13} \mathrm{C}$ NMR spectra were recorded on Bruker AVANCE II 300 spectrometer (300.13 and $75.48 \mathrm{MHz}$, respectively) in $\mathrm{CDCl}_{3}$. Chemical shifts were reported in parts per million (ppm), and the residual solvent peak was used as an internal reference: ${ }^{1} \mathrm{H}\left(\mathrm{CDCl}_{3} \delta=7.26 \mathrm{ppm}\right),{ }^{13} \mathrm{C}\left(\mathrm{CDCl}_{3}\right.$ $\delta=77.16 \mathrm{ppm}$ ). Multiplicity was indicated as follows: $s$ (singlet), $d$ (doublet), $t$ (triplet), q (quartet), $\mathrm{m}$ (multiplet).

High resolution mass spectra (HR-MS) were measured on a Bruker micrOTOF II instrument using electrospray ionization (ESI). ${ }^{1}$ The measurements were performed in a positive ion mode (interface capillary voltage $-4500 \mathrm{~V}$ ); mass range from m/z 50 to m/z $3000 \mathrm{Da}$; external calibration with Electrospray Calibrant Solution (Fluka). A syringe injection was used for all acetonitrile solutions (flow rate $3 \mu \mathrm{L} / \mathrm{min}$ ). Nitrogen was applied as a dry gas; interface temperature was set at $180^{\circ} \mathrm{C}$.

Reaction mixtures (Table 1) were additionally analysed by GC. The GC analysis of the reaction mixtures was carried out on a gas chromatograph equipped by a flame ionization detector and a nonpolar capillary column (stationary phase: dimethyl polysiloxane, film thickness: $1.0 \mu \mathrm{m}$, length: $30 \mathrm{~m}$, inner diameter: $0.32 \mathrm{~mm}$ ). Evaporating chamber temperature for $\mathrm{GC}$ analysis was $250{ }^{\circ} \mathrm{C}$, column chamber temperature was gradually increased from $70{ }^{\circ} \mathrm{C}$ to $250{ }^{\circ} \mathrm{C}$ at a rate of $30{ }^{\circ} \mathrm{C} / \mathrm{min}$. Valerophenone $\mathrm{C}_{6} \mathrm{H}_{5} \mathrm{C}(\mathrm{O}) \mathrm{C}_{4} \mathrm{H}_{9}$ was used as an internal standard.

\section{General Procedure for Cyclic Voltammetry}

Cyclic voltammetry (CV) was implemented on an IPC-Pro M computer-assisted potentiostat manufactured by «Econix» (scan rate error 1.0\%; potential setting $0.25 \mathrm{mV}$; scan rate $100 \mathrm{mV} / \mathrm{s}$ ). The experiments were performed in a $10 \mathrm{~mL}$ fiveneck glass conic electrochemical cell with a water jacket for thermostatting. CV curves were recorded using a three-electrode scheme. In a typical case, $5 \mathrm{~mL}$ of a solution was utilized. The working electrode was a disc glassy-carbon electrode $(\mathrm{d}=3 \mathrm{~mm})$. A platinum wire served as an auxiliary electrode. $\mathrm{An} \mathrm{Ag} / \mathrm{AgNO}_{3}$ electrode was used as the reference electrode and was linked to the solution by a porous glass diaphragm. The solutions were kept under thermally controlled conditions at $15 \pm 0.5^{\circ} \mathrm{C}$ and deaerated by bubbling argon. Electrochemical experiments were performed under an argon atmosphere. The working electrode was polished before recording each CV curve.

The TLC analysis was carried out on standard silica gel chromatography plates (DC-Fertigfolien ALUGRAM ${ }^{R}$ Xtra SIL G/UV254). Column chromatography was performed using silica gel (0.060$0.200 \mathrm{~mm}, 60 \mathrm{~A}$, CAS 7631-86-9, Acros).

$\mathrm{LiClO}_{4}, \mathrm{NaBF}_{4}, n-\mathrm{Bu}_{4} \mathrm{NClO}_{4}, n-\mathrm{Bu}_{4} \mathrm{NBF}_{4}, \mathrm{CF}_{3} \mathrm{SO}_{2} \mathrm{Na}$ were purchased from commercial sources and was used as is. All solvents were distilled before use using standard procedures.

\section{Enol acetates data}

Starting enol acetates 1 were prepared according to literature. ${ }^{2-3}$ 
<smiles>C=C(OC(C)=O)c1ccccc1</smiles>

${ }^{1} \mathrm{H}$ NMR (300 MHz, $\left.\mathrm{CDCl}_{3}, \delta\right): 7.52-7.44(\mathrm{~m}, 2 \mathrm{H}), 7.40-7.31(\mathrm{~m}, 3 \mathrm{H}), 5.49(\mathrm{~d}, \mathrm{~J}=2.0 \mathrm{~Hz}, 1 \mathrm{H})$, $5.04(\mathrm{~d}, J=2.0 \mathrm{~Hz}, 1 \mathrm{H}), 2.28(\mathrm{~s}, 3 \mathrm{H})$.

${ }^{13} \mathrm{C}$ NMR (75 MHz, $\left.\mathrm{CDCl}_{3}, \delta\right):$ 169.2, 153.1, 134.4, 129.1, 128.6, 125.0, 102.2, 21.1.

1-(p-Tolyl)vinyl acetate, $1 b^{5}$<smiles>C=C(OC(C)=O)c1ccc(C)cc1</smiles>

${ }^{1} \mathrm{H}$ NMR (300 MHz, $\left.\mathrm{CDCl}_{3}, \delta\right): 7.36$ (d, $\left.J=8.2 \mathrm{~Hz}, 2 \mathrm{H}\right), 7.16(\mathrm{~d}, J=8.2 \mathrm{~Hz}, 2 \mathrm{H}), 5.43(\mathrm{~d}, J=2.1$ $\mathrm{Hz}, 1 \mathrm{H}), 4.98(\mathrm{~d}, J=2.1 \mathrm{~Hz}, 1 \mathrm{H}), 2.35$ (s, 3H), 2.27 (s, 3H).

${ }^{13} \mathrm{C}$ NMR $\left(75 \mathrm{MHz}, \mathrm{CDCl}_{3}, \delta\right): 169.2,153.1,139.1,131.6,129.3,124.9,101.3,21.3,21.1$.

\section{1-(4-Methoxyphenyl)vinyl acetate, 1c ${ }^{4}$}<smiles>C=C(OC(C)=O)c1ccc(OC)cc1</smiles>

${ }^{1} \mathrm{H}$ NMR (300 MHz, $\left.\mathrm{CDCl}_{3}, \delta\right): 7.46-7.35(\mathrm{~m}, 2 \mathrm{H}), 6.87(\mathrm{~d}, J=8.9 \mathrm{~Hz}, 2 \mathrm{H}), 5.35(\mathrm{~d}, J=2.1 \mathrm{~Hz}$, $1 \mathrm{H}), 4.92(\mathrm{~d}, J=2.1 \mathrm{~Hz}, 1 \mathrm{H}), 3.81(\mathrm{~s}, 3 \mathrm{H}), 2.27$ (s, 3H).

${ }^{13} \mathrm{C} \mathrm{NMR}\left(75 \mathrm{MHz}, \mathrm{CDCl}_{3}, \delta\right): 169.2,160.3,152.9,127.0,126.4,114.0,100.4,55.4,21.1$.

1-(4-Chlorophenyl)vinyl acetate, $1 \mathrm{~d}^{6}$<smiles>C=C(OC(C)=O)c1ccc(Cl)cc1</smiles>

${ }^{1} \mathrm{H}$ NMR (300 MHz, $\left.\mathrm{CDCl}_{3}, \delta\right): 7.42-7.36(\mathrm{~m}, 2 \mathrm{H}), 7.34-7.28(\mathrm{~m}, 2 \mathrm{H}), 5.45(\mathrm{~d}, \mathrm{~J}=2.3 \mathrm{~Hz}, 1 \mathrm{H})$, $5.04(\mathrm{~d}, J=2.3 \mathrm{~Hz}, 1 \mathrm{H}), 2.27(\mathrm{~s}, 3 \mathrm{H})$.

${ }^{13} \mathrm{C}$ NMR $\left(75 \mathrm{MHz} \mathrm{CDCl}_{3}, \delta\right): 169.1,152.0,134.9,132.9,128.9,126.3,102.8,21.1$.

\section{1-(4-Fluorophenyl)vinyl acetate, $1 \mathrm{e}^{7}$}<smiles>C=C(OC(C)=O)c1ccc(F)cc1</smiles>

${ }^{1} \mathrm{H}$ NMR (300 MHz, $\left.\mathrm{CDCl}_{3}, \delta\right): 7.51-7.36(\mathrm{~m}, 2 \mathrm{H}), 7.03(\mathrm{t}, J=8.7 \mathrm{~Hz}, 2 \mathrm{H}), 5.40(\mathrm{~d}, J=2.2 \mathrm{~Hz}$, $1 \mathrm{H}), 5.01(\mathrm{~d}, \mathrm{~J}=2.2 \mathrm{~Hz}, 1 \mathrm{H}), 2.27(\mathrm{~s}, 3 \mathrm{H})$.

${ }^{13} \mathrm{C}$ NMR (75 MHz, $\left.\mathrm{CDCl}_{3}, \delta\right): 169.1,163.2\left(\mathrm{~d},{ }^{1} \mathrm{~J}_{\mathrm{CF}}=248.8 \mathrm{~Hz}\right), 152.2,131.1\left(\mathrm{~d},{ }^{3} \mathrm{~J}_{\mathrm{CF}}=9.3 \mathrm{~Hz}\right)$, $130.7\left(\mathrm{~d},{ }^{4} \mathrm{~J}_{\mathrm{CF}}=3.4 \mathrm{~Hz}\right), 126.9\left(\mathrm{~d},{ }^{3} \mathrm{~J}_{\mathrm{CF}}=8.3 \mathrm{~Hz}\right), 115.7\left(\mathrm{~d},{ }^{2} \mathrm{~J}_{\mathrm{CF}}=21.9 \mathrm{~Hz}\right), 102.1\left(\mathrm{~d},{ }^{6} \mathrm{~J}_{\mathrm{CF}}=1.6\right.$ $\mathrm{Hz}), 21.1$. 
${ }^{19} \mathrm{~F}$ NMR (282 $\left.\mathrm{MHz}, \mathrm{CDCl}_{3}, \delta\right):-112.35$ (tt, $\left.J=8.5,5.3 \mathrm{~Hz}\right)$.

1-(4-Bromophenyl)vinyl acetate, $1 f^{8}$<smiles>C=C(OC(C)=O)c1ccc(Br)cc1</smiles>

${ }^{1} \mathrm{H}$ NMR (300 MHz, $\left.\mathrm{CDCl}_{3}, \delta\right): 7.48(\mathrm{~d}, J=8.5 \mathrm{~Hz}, 2 \mathrm{H}), 7.33(\mathrm{~d}, J=8.5 \mathrm{~Hz}, 2 \mathrm{H}), 5.47$ (d, J = 2.1 $\mathrm{Hz}, 1 \mathrm{H}), 5.06(\mathrm{~d}, J=2.1 \mathrm{~Hz}, 1 \mathrm{H}), 2.27(\mathrm{~s}, 3 \mathrm{H})$.

${ }^{13} \mathrm{C} \mathrm{NMR}\left(75 \mathrm{MHz}_{\mathrm{CDCl}}\right.$, $\left.\mathrm{\delta}\right):$ 169.0, 152.2, 133.5, 131.9, 126.6, 123.2, 102.9, 21.1.

$1 H$-Inden-3-yl acetate, $\mathbf{1 g}^{9}$<smiles>CC(=O)OC1=CCc2ccccc21</smiles>

${ }^{1} \mathrm{H}$ NMR (300 MHz, $\left.\mathrm{CDCl}_{3}, \delta\right): 7.45(\mathrm{~d}, J=7.2 \mathrm{~Hz}, 1 \mathrm{H}), 7.34-7.22(\mathrm{~m}, 3 \mathrm{H}), 6.34$ (t, $J=2.2 \mathrm{~Hz}$, $1 \mathrm{H}), 3.42(\mathrm{~d}, J=2.2 \mathrm{~Hz}, 2 \mathrm{H}), 2.34(\mathrm{~s}, 3 \mathrm{H})$.

${ }^{13} \mathrm{C} \mathrm{NMR}$ (75 MHz, $\left.\mathrm{CDCl}_{3}, \delta\right): 168.4,149.2,141.9,139.1,126.4,125.8,124.2,118.1,115.7,35.1$, 21.3.

\section{3,4-Dihydronaphthalen-1-yl acetate, $1 \mathrm{~h}^{4}$}<smiles>CC(=O)OC1=CCCc2ccccc21</smiles>

${ }^{1} \mathrm{H}$ NMR (300 MHz, $\left.\mathrm{CDCl}_{3}, \delta\right): 7.21-7.06(\mathrm{~m}, 4 \mathrm{H}), 5.71(\mathrm{t}, J=4.7 \mathrm{~Hz}, 1 \mathrm{H}), 2.87$ (t, J=8.1 Hz, $2 \mathrm{H}), 2.45(\mathrm{td}, J=8.1,4.7 \mathrm{~Hz}, 2 \mathrm{H}), 2.30(\mathrm{~s}, 3 \mathrm{H})$.

${ }^{13} \mathrm{C} \mathrm{NMR}\left(75 \mathrm{MHz}_{\mathrm{CDCl}}\right.$, $\left.\mathrm{\delta}\right):$ 169.4, 145.7, 136.5, 130.5, 128.0, 127.7, 126.5, 120.8, 115.6, 27.6, 22.1, 21.0.

(Z)-1-Phenylprop-1-en-1-yl acetate, 1 i 10<smiles>CC=C(OC(C)=O)c1ccccc1</smiles>

${ }^{1} \mathrm{H}$ NMR (300 MHz, $\left.\mathrm{CDCl}_{3}, \delta\right): 7.45-7.22(\mathrm{~m}, 5 \mathrm{H}), 5.91(\mathrm{q}, J=7.0 \mathrm{~Hz}, 1 \mathrm{H}), 2.31$ (s, 3H), $1.73(\mathrm{~d}$, $J=7.0 \mathrm{~Hz}, 3 \mathrm{H})$.

${ }^{13} \mathrm{C}$ NMR $\left(75 \mathrm{MHz}, \mathrm{CDCl}_{3}, \delta\right): 168.7,147.1,135.1,128.6,128.1,124.4,112.7,20.7,11.6$.

1-(Naphthalen-2-yl)vinyl acetate, 1j 4

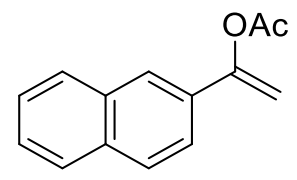

${ }^{1} \mathrm{H}$ NMR (300 MHz, DMSO-d6, ס): 8.03 - 7.87 (m, 4H), $7.74(\mathrm{dd}, J=8.7,1.9 \mathrm{~Hz}, 1 \mathrm{H}), 7.58-7.49$ $(\mathrm{m}, 2 \mathrm{H}), 5.85(\mathrm{~d}, J=2.3 \mathrm{~Hz}, 1 \mathrm{H}), 5.13(\mathrm{~d}, J=2.3 \mathrm{~Hz}, 1 \mathrm{H}), 2.35(\mathrm{~s}, 3 \mathrm{H})$. 
${ }^{13} \mathrm{C}$ NMR (75 MHz, DMSO- $\left.d_{6}, \delta\right): 169.0,152.1,132.9,132.7,131.1,128.4,128.2,127.5,126.7$, 126.6, 123.4, 122.6, 103.4, 20.8.

\section{Cyclopent-1-en-1-yl acetate, $1 \mathrm{k}^{11}$}

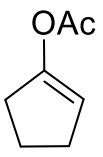

${ }^{1} \mathrm{H}$ NMR (300 MHz, $\left.\mathrm{CDCl}_{3}, \delta\right): 5.42-5.32(\mathrm{~m}, 1 \mathrm{H}), 2.47-2.29(\mathrm{~m}, 4 \mathrm{H}), 2.10(\mathrm{~s}, 3 \mathrm{H}), 1.92$ (quint, $J=7.5 \mathrm{~Hz}, 2 \mathrm{H})$.

${ }^{13} \mathrm{C}$ NMR $\left(75 \mathrm{MHz} \mathrm{CDCl}_{3}, \delta\right): 168.7,151.1,113.1,31.0,28.7,21.1$.

Cyclohex-1-en-1-yl acetate, $1{ }^{12}$<smiles>CC(=O)OC1=CCCCC1</smiles>

${ }^{1} \mathrm{H}$ NMR (300 MHz, $\left.\mathrm{CDCl}_{3}, \delta\right): 5.33(\mathrm{t}, J=3.8 \mathrm{~Hz}, 1 \mathrm{H}), 2.13-2.04(\mathrm{~m}, 7 \mathrm{H}), 1.76-1.66(\mathrm{~m}, 2 \mathrm{H})$, $1.61-1.52(\mathrm{~m}, 2 \mathrm{H})$.

${ }^{13} \mathrm{C}$ NMR $\left(75 \mathrm{MHz} \mathrm{CDCl}_{3}, \delta\right):$ 169.5, 148.5, 114.1, 26.9, 23.7, 22.7, 21.8, 21.1.

4-(Tert-butyl)cyclohex-1-en-1-yl acetate, 1m 4<smiles>CC(=O)OC1=CCC(C(C)(C)C)CC1</smiles>

${ }^{1} \mathrm{H}$ NMR (300 MHz, $\left.\mathrm{CDCl}_{3}, \delta\right): 5.33(\mathrm{dt}, J=5.0,2.2 \mathrm{~Hz}, 1 \mathrm{H}), 2.35-2.18(\mathrm{~m}, 1 \mathrm{H}), 2.16-2.01(\mathrm{~m}$, $2 \mathrm{H}), 2.09(\mathrm{~s}, 3 \mathrm{H}), 1.95-1.80(\mathrm{~m}, 2 \mathrm{H}), 1.43-1.23(\mathrm{~m}, 2 \mathrm{H}), 0.87(\mathrm{~s}, 9 \mathrm{H})$.

${ }^{13} \mathrm{C} \mathrm{NMR}\left(75 \mathrm{MHz}, \mathrm{CDCl}_{3}, \delta\right):$ 169.6, 148.4, 114.1, 43.5, 32.3, 28.0, 27.4, 25.1, 24.1, 21.2.

Cyclohept-1-en-1-yl acetate, 1n 4

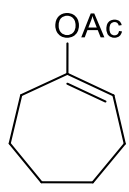

${ }^{1} \mathrm{H}$ NMR (300 MHz, $\left.\mathrm{CDCl}_{3}, \delta\right): 5.52-5.38(\mathrm{~m}, 1 \mathrm{H}), 2.37-2.26(\mathrm{~m}, 2 \mathrm{H}), 2.16-2.03(\mathrm{~m}, 2 \mathrm{H}), 2.09$ $(\mathrm{s}, 3 \mathrm{H}), 1.75-1.55(\mathrm{~m}, 6 \mathrm{H})$.

${ }^{13} \mathrm{C}$ NMR (75 MHz, CDCl3, ठ): 169.9, 153.3, 118.1, 33.2, 31.1, 27.1, 25.4, 25.3, 21.1.

Cyclododec-1-en-1-yl acetate, 10<smiles>CC(=O)O/C1=C/CCCCCCCCCC1</smiles>

a mixture of $E$ - and $Z$ - isomers. 
${ }^{1} \mathrm{H}$ NMR $\left(300 \mathrm{MHz}, \mathrm{CDCl}_{3}, \delta\right): 5.11(\mathrm{t}, J=7.6 \mathrm{~Hz}, 0.7 \mathrm{H}), 5.06(\mathrm{t}, J=8.2 \mathrm{~Hz}, 0.3 \mathrm{H}), 2.34(\mathrm{t}, J=6.7$ $\mathrm{Hz}, 0.7 \mathrm{H}), 2.29-2.22(\mathrm{~m}, 1 \mathrm{H}), 2.16(\mathrm{~s}, 2.1 \mathrm{H}), 2.06-2.15(\mathrm{~m}, 0.7 \mathrm{H}), 2.10(\mathrm{~s}, 0.9 \mathrm{H}), 2.03-1.92$ $(\mathrm{m}, 1.6 \mathrm{H}), 1.56-1.21(\mathrm{~m}, 16 \mathrm{H})$.

${ }^{13} \mathrm{C}$ NMR $\left(75 \mathrm{MHz}, \mathrm{CDCl}_{3}, \delta\right): 169.9,169.2,149.0,147.8,119.3,119.1,32.9,27.1,26.4,26.0$, 25.7, 25.6, 25.1, 25.0, 24.7, 24.63, 24.61, 24.5, 24.4, 24.2, 24.0, 23.7, 23.5, 22.3, 22.2, 21.1, 20.9. HRMS (ESI-TOF) $\mathrm{m} / \mathrm{z}\left[\mathrm{M}+\mathrm{NH}_{4}\right]^{+}$. Calcd for $\left[\mathrm{C}_{14} \mathrm{H}_{28} \mathrm{NO}_{2}\right]^{+}: 242.2115$. Found: 242.2110.

\section{Experimental procedures for Table 1}

\section{Experimental procedure for Table 1, entries 1-5}

An undivided cell was equipped with a platinum plate $\left(3 \mathrm{~cm}^{2}\right)$ anode and a platinum plate cathode $\left(3 \mathrm{~cm}^{2}\right)$ and connected to a DC regulated power supply. The solution of enol acetate $1 \mathrm{a}(1.0 \mathrm{mmol}$, $162.0 \mathrm{mg}$ ), $\mathrm{CF}_{3} \mathrm{SO}_{2} \mathrm{Na}\left(2.0 \mathrm{mmol}, 312.0 \mathrm{mg}\right.$ ) and supporting electrolyte $\mathrm{LiClO}_{4}, \mathrm{NaBF}_{4}, n-$ $\mathrm{Bu}_{4} \mathrm{NClO}_{4}$, or $n$-Bu4NBF $4(53.2-170.9 \mathrm{mg}, 0.5 \mathrm{mmol})$ in $10 \mathrm{~mL}$ of $\mathrm{CH}_{3} \mathrm{CN} / \mathrm{H}_{2} \mathrm{O}$ (8:2) was electrolyzed using constant current conditions $\left(\mathrm{j}=6.7-13.3 \mathrm{~mA} / \mathrm{cm}^{2}\right)$ at $20-25^{\circ} \mathrm{C}$ under magnetic stirring for 120-241 min with I $=20-40 \mathrm{~mA}$. Then electrodes were washed with $\mathrm{CH}_{2} \mathrm{Cl}_{2}(2 \times 20$ $\mathrm{mL})$. The combined organic phases were washed with brine $(3 \times 10 \mathrm{~mL})$, dried over $\mathrm{MgSO}_{4}$, filtered and concentrated under reduced pressure using a rotary evaporator $(15-20 \mathrm{mmHg}$ ), (bath temperature, ca. $\left.20-25^{\circ} \mathrm{C}\right)$. Product $2 \mathrm{a}$ was isolated by chromatography on $\mathrm{SiO}_{2}(\mathrm{PE}: \mathrm{EtOAc}=$ from $20: 1$ to $2: 1$ ).

\section{Experimental procedure for Table 1, entry 6}

An undivided cell was equipped with a platinum plate anode $\left(3 \mathrm{~cm}^{2}\right)$ and a platinum plate cathode $\left(3 \mathrm{~cm}^{2}\right)$ and connected to a DC regulated power supply. The solution of enol acetate $1 \mathrm{a}(1.0 \mathrm{mmol}$, $162.0 \mathrm{mg}), \mathrm{CF}_{3} \mathrm{SO}_{2} \mathrm{Na}(2.0 \mathrm{mmol}, 312.0 \mathrm{mg})$ and supporting electrolyte $n$-Bu4NBF $(164.7 \mathrm{mg}$, $0.5 \mathrm{mmol}$ ) in $10 \mathrm{~mL}$ of $\mathrm{CH}_{3} \mathrm{CN} / \mathrm{H}_{2} \mathrm{O}(8: 2)$ was stirred at $20-25^{\circ} \mathrm{C}$ for $241 \mathrm{~min}$. The target product 2a was not detected by TLC during and after the experiment.

\section{Experimental procedure for Table 1, entries 7-20}

An undivided cell was equipped with a platinum plate, glassy carbon, or graphite anode $\left(3 \mathrm{~cm}^{2}\right)$ and a platinum plate, stainless steel, glassy carbon, or copper cathode $\left(3 \mathrm{~cm}^{2}\right)$ and connected to a DC regulated power supply. The solution of enol acetate $1 \mathrm{a}(1.0 \mathrm{mmol}, 162.0 \mathrm{mg})$ and $\mathrm{CF}_{3} \mathrm{SO}_{2} \mathrm{Na}$ (1.0-3.0 mmol, $156.0-468.0 \mathrm{mg}$ ) in $10 \mathrm{~mL}$ of $\mathrm{CH}_{3} \mathrm{CN} / \mathrm{H}_{2} \mathrm{O}$ (8:2), DMSO/ $\mathrm{H}_{2} \mathrm{O}$ (8:2), $\mathrm{THF} / \mathrm{H}_{2} \mathrm{O}$ (8:2), $\mathrm{CH}_{3} \mathrm{CN}$ or $\mathrm{CH}_{3} \mathrm{OH}$ was electrolyzed using constant current conditions $(\mathrm{j}=6.7$ $\mathrm{mA} / \mathrm{cm}^{2}$ ) at 20-25 ${ }^{\circ} \mathrm{C}$ under magnetic stirring for 177-402 min with I $=20 \mathrm{~mA}$. Then electrodes were washed with $\mathrm{CH}_{2} \mathrm{Cl}_{2}(2 \times 20 \mathrm{~mL})$. The combined organic phases were washed with brine (3 $\times 10 \mathrm{~mL}$ ), dried over $\mathrm{MgSO}_{4}$, filtered and concentrated under reduced pressure using a rotary evaporator $\left(15-20 \mathrm{mmHg}\right.$ ), (bath temperature, ca. $20-25{ }^{\circ} \mathrm{C}$ ). Product $2 \mathbf{a}$ was isolated by chromatography on $\mathrm{SiO}_{2}(\mathrm{PE}: \mathrm{EtOAc}=$ from 20:1 to 2:1). 
The conversion of enol acetate 1a was $>95 \%$ in most experiments of Table 1 . Acetophenone, 2 hydroxyacetophenone, and 2-acetoxyacetophenone were observed as side products in yields from trace to low $(<10 \%)$.

\section{Electrolysis of silyl enol ether with $\mathrm{CF}_{3} \mathrm{SO}_{2} \mathrm{Na}$}

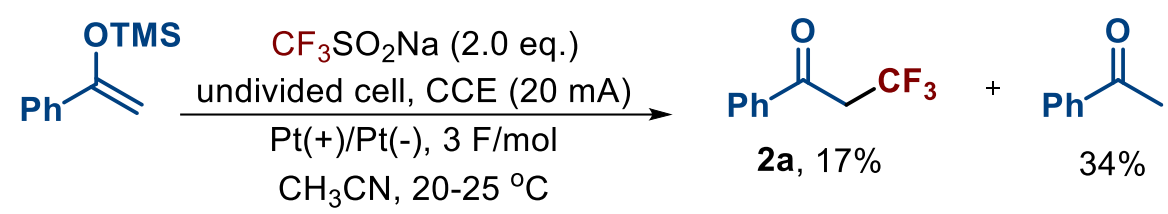

An undivided cell was equipped with a platinum plate anode $\left(3 \mathrm{~cm}^{2}\right)$ and a platinum plate cathode $\left(3 \mathrm{~cm}^{2}\right)$ and connected to a DC regulated power supply. The solution of silyl enol ether (1.0 mmol) and $\mathrm{CF}_{3} \mathrm{SO}_{2} \mathrm{Na}(2.0 \mathrm{mmol}, 312.0 \mathrm{mg})$ in $10 \mathrm{~mL}$ of $\mathrm{CH}_{3} \mathrm{CN}(10 \mathrm{~mL})$ was electrolyzed using constant current conditions $\left(\mathrm{j}=6.7 \mathrm{~mA} / \mathrm{cm}^{2}\right)$ at $20-25{ }^{\circ} \mathrm{C}$ under magnetic stirring for 241 min with I $=20$ $\mathrm{mA}$. Then electrodes were washed with $\mathrm{CH}_{2} \mathrm{Cl}_{2}(2 \times 20 \mathrm{~mL})$. The combined organic phases were washed with brine $(3 \times 10 \mathrm{~mL})$, dried over $\mathrm{MgSO}_{4}$, filtered and concentrated under reduced pressure using a rotary evaporator $\left(15-20 \mathrm{mmHg}\right.$ ), (bath temperature, ca. $20-25^{\circ} \mathrm{C}$ ). Product $2 \mathbf{a}$ and acetophenone were isolated by chromatography on $\mathrm{SiO}_{2}(\mathrm{PE}: \mathrm{EtOAC}=$ from 20:1 to 2:1).

\section{General Experimental Procedure for Scheme 2}

An undivided cell was equipped with a platinum plate anode $\left(3 \mathrm{~cm}^{2}\right)$ and a platinum plate cathode $\left(3 \mathrm{~cm}^{2}\right)$ and connected to a DC regulated power supply. The solution of enol acetate $1(1.0 \mathrm{mmol})$ and $\mathrm{CF}_{3} \mathrm{SO}_{2} \mathrm{Na}(2.0 \mathrm{mmol}, 312.0 \mathrm{mg})$ in $10 \mathrm{~mL}$ of $\mathrm{CH}_{3} \mathrm{CN} / \mathrm{H}_{2} \mathrm{O}$ (8:2) was electrolyzed using constant current conditions $\left(j=6.7 \mathrm{~mA} / \mathrm{cm}^{2}\right)$ at $20-25^{\circ} \mathrm{C}$ under magnetic stirring for 241 min with $\mathrm{I}=20 \mathrm{~mA}$. Then electrodes were washed with $\mathrm{CH}_{2} \mathrm{Cl}_{2}(2 \times 20 \mathrm{~mL})$. The combined organic phases were washed with brine $(3 \times 10 \mathrm{~mL})$, dried over $\mathrm{MgSO}_{4}$, filtered and concentrated under reduced pressure using a rotary evaporator $\left(15-20 \mathrm{mmHg}\right.$ ), (bath temperature, ca. $20-25^{\circ} \mathrm{C}$ ). Product 2 was isolated by chromatography on $\mathrm{SiO}_{2}(\mathrm{PE}: \mathrm{EtOAc}=$ from 20:1 to 2:1).

\section{3,3,3-Trifluoro-1-phenylpropan-1-one, $2 \mathrm{a}^{3}$}

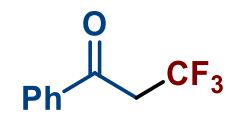

Yield $85 \%$ (160.0 mg, $0.85 \mathrm{mmol}$ ). White crystals, $\mathrm{mp}=37-38{ }^{\circ} \mathrm{C}$ (lit. $\left.37{ }^{\circ} \mathrm{C}^{13}\right) . \mathrm{R}_{\mathrm{f}}=0.79$ (PE:EtOAc $=10: 1)$.

${ }^{1} \mathrm{H}$ NMR $\left(300 \mathrm{MHz}, \mathrm{CDCl}_{3}, \delta\right): 7.93(\mathrm{~d}, J=8.6 \mathrm{~Hz}, 2 \mathrm{H}), 7.63(\mathrm{t}, J=7.5 \mathrm{~Hz}, 1 \mathrm{H}), 7.50$ (t, $J=7.5$ $\mathrm{Hz}, 2 \mathrm{H}$ ), 3.80 (q, $J=10.0 \mathrm{~Hz}, 2 \mathrm{H}$ ). 
${ }^{13} \mathrm{C} \mathrm{NMR}\left(75 \mathrm{MHz}, \mathrm{CDCl}_{3}, \delta\right): 189.9$ (q, $\left.{ }^{3} \mathrm{~J}_{\mathrm{CF}}=2.3 \mathrm{~Hz}\right), 136.0,134.3,129.1,128.5,124.2$ (q, ${ }^{1} \mathrm{~J}_{\mathrm{CF}}$ $=277.0 \mathrm{~Hz}), 42.2\left(\mathrm{q},{ }^{2} \mathrm{~J}_{\mathrm{CF}}=28.2 \mathrm{~Hz}\right)$.

${ }^{19} \mathrm{~F} \mathrm{NMR}\left(282 \mathrm{MHz}, \mathrm{CDCl}_{3}, \delta\right):-62.84(\mathrm{t}, J=10.0 \mathrm{~Hz}, 3 \mathrm{~F})$.

3,3,3-Trifluoro-1-(p-tolyl)propan-1-one, 2b ${ }^{14}$<smiles>Cc1ccc(C(=O)CC(F)(F)F)cc1</smiles>

Yield $77 \%(155.7 \mathrm{mg}, 0.77 \mathrm{mmol})$. White crystals, $\mathrm{mp}=53-54{ }^{\circ} \mathrm{C}$ (lit. $\left.54-55{ }^{\circ} \mathrm{C}^{14}\right) . \mathrm{R}_{\mathrm{f}}=0.83$ (PE:EtOAc $=10: 1)$.

${ }^{1} \mathrm{H}$ NMR (300 MHz, CDCl $\left.3, \delta\right): 7.82(\mathrm{~d}, J=8.2 \mathrm{~Hz}, 2 \mathrm{H}), 7.29(\mathrm{~d}, J=8.2 \mathrm{~Hz}, 2 \mathrm{H}), 3.76(\mathrm{q}, J=10.0$ $\mathrm{Hz}, 2 \mathrm{H}), 2.42(\mathrm{~s}, 3 \mathrm{H})$.

${ }^{13} \mathrm{C}$ NMR $\left(75 \mathrm{MHz} \mathrm{CDCl}_{3}, \delta\right): 189.5\left(\mathrm{q},{ }^{3} \mathrm{~J}_{\mathrm{CF}}=2.5 \mathrm{~Hz}\right), 145.6,133.5,129.7,128.6,124.2$ (q, ${ }^{1} \mathrm{JCF}_{\mathrm{C}}$ $=265.7 \mathrm{~Hz}), 42.0\left(\mathrm{q},{ }^{2} \mathrm{~J}_{\mathrm{CF}}=28.1 \mathrm{~Hz}\right), 21.7$.

${ }^{19} \mathrm{~F}$ NMR $\left(282 \mathrm{MHz}, \mathrm{CDCl}_{3}, \delta\right):-62.06$ (t, $\left.J=10.0 \mathrm{~Hz}, 3 \mathrm{~F}\right)$.

3,3,3-Trifluoro-1-(4-methoxyphenyl)propan-1-one, 2c ${ }^{14}$<smiles>COc1ccc(C(=O)CC(F)(F)F)cc1</smiles>

Yield $71 \%(155.0 \mathrm{mg}, 0.71 \mathrm{mmol})$. White crystals, $\mathrm{mp}=41-42{ }^{\circ} \mathrm{C}$ (lit. $\left.41-42{ }^{\circ} \mathrm{C}^{14}\right) . \mathrm{R}_{\mathrm{f}}=0.75$ $(\mathrm{PE}: \mathrm{EtOAC}=10: 1)$.

${ }^{1} \mathrm{H}$ NMR (300 MHz, $\left.\mathrm{CDCl}_{3}, \delta\right): 7.89$ (d, J = 8.9 Hz, 2H), 6.94 (d, J=8.9 Hz, 2H), 3.86 (s, 3H), 3.73 (q, $J=10.0 \mathrm{~Hz}, 2 \mathrm{H})$.

${ }^{13} \mathrm{C} \mathrm{NMR}\left(75 \mathrm{MHz} \mathrm{CDCl}_{3}, \delta\right): 188.3\left(\mathrm{q},{ }^{3} \mathrm{~J}_{\mathrm{CF}}=2.5 \mathrm{~Hz}\right), 164.5,130.9,129.0,124.3$ (q, ${ }^{1} \mathrm{JCF}_{\mathrm{CF}} 276.9$ $\mathrm{Hz}), 114.2,55.6,41.8\left(\mathrm{q},{ }^{2} \mathrm{~J}_{\mathrm{CF}}=27.9 \mathrm{~Hz}\right)$.

${ }^{19} \mathrm{~F} \mathrm{NMR}\left(282 \mathrm{MHz}, \mathrm{CDCl}_{3}, \delta\right):-62.81(\mathrm{t}, J=10.0 \mathrm{~Hz}, 3 \mathrm{~F})$.

1-(4-Chlorophenyl)-3,3,3-trifluoropropan-1-one, 2d ${ }^{14}$<smiles>O=C(CC(F)(F)F)c1ccc(Cl)cc1</smiles>

Yield $63 \%$ (140.2 mg, $0.63 \mathrm{mmol})$. White crystals, $\mathrm{mp}=56-57{ }^{\circ} \mathrm{C}$ (lit. $\left.55-56{ }^{\circ} \mathrm{C}^{14}\right) . \mathrm{R}_{\mathrm{f}}=0.69$ (PE:EtOAC $=10: 1)$.

${ }^{1} \mathrm{H}$ NMR (300 MHz, $\left.\mathrm{CDCl}_{3}, \delta\right): 7.89$ (d, J = 8.5 Hz, 2H), 7.49 (d, J=8.5 Hz, 2H), 3.80 (q, J = 9.9 $\mathrm{Hz}, 2 \mathrm{H})$.

${ }^{13} \mathrm{C} \mathrm{NMR}\left(75 \mathrm{MHz}, \mathrm{CDCl}_{3}, \delta\right): 188.7$ (q, $\left.{ }^{3} \mathrm{JCF}_{\mathrm{CF}} 2.3 \mathrm{~Hz}\right), 141.0,134.2,129.9,129.4,124.0$ (q, ${ }^{1} \mathrm{JCF}_{\mathrm{CF}}$ $=277.0 \mathrm{~Hz}), 42.2\left(\mathrm{q},{ }^{2} \mathrm{~J}_{\mathrm{CF}}=28.4 \mathrm{~Hz}\right)$.

${ }^{19} \mathrm{~F} \mathrm{NMR}\left(282 \mathrm{MHz}, \mathrm{CDCl}_{3}, \delta\right):-62.05(\mathrm{t}, J=9.9 \mathrm{~Hz}, 3 \mathrm{~F})$. 
3,3,3-Trifluoro-1-(4-fluorophenyl)propan-1-one, $2 e^{15}$<smiles>O=C(CC(F)(F)F)c1ccc(F)cc1</smiles>

Yield $79 \%$ (162.8 mg, $0.79 \mathrm{mmol}$ ). White crystals, $\mathrm{mp}=33{ }^{\circ} \mathrm{C}$ (lit. $\left.31.5-32.4{ }^{\circ} \mathrm{C}^{16}\right) . \mathrm{R}_{\mathrm{f}}=0.65$ (PE:EtOAc $=10: 1)$.

${ }^{1} \mathrm{H}$ NMR (300 MHz, $\left.\mathrm{CDCl}_{3}, \delta\right): 8.02-7.88(\mathrm{~m}, 2 \mathrm{H}), 7.22-7.10(\mathrm{~m}, 2 \mathrm{H}), 3.78(\mathrm{q}, \mathrm{J}=10.0 \mathrm{~Hz}, 2 \mathrm{H})$. ${ }^{13} \mathrm{C} \mathrm{NMR}\left(75 \mathrm{MHz}, \mathrm{CDCl}_{3}, \delta\right): 188.4$ (q, $\left.{ }^{3} \mathrm{~J}_{\mathrm{CF}}=2.5 \mathrm{~Hz}\right), 166.5$ (d, $\left.{ }^{1} \mathrm{~J}_{\mathrm{CF}}=256.9 \mathrm{~Hz}\right), 132.4$ (dq, ${ }^{4} \mathrm{JCF}$ = 3.0, 1.6 Hz), $131.3\left(\mathrm{~d},{ }^{3} J_{\mathrm{CF}}=9.6 \mathrm{~Hz}\right), 124.1\left(\mathrm{q},{ }^{1} J_{\mathrm{CF}}=276.9 \mathrm{~Hz}\right), 116.3\left(\mathrm{~d},{ }^{2} J_{\mathrm{CF}}=22.2 \mathrm{~Hz}\right), 42.1$ $\left(q,{ }^{2} J_{C F}=28.3 \mathrm{~Hz}\right)$.

${ }^{19} \mathrm{~F} \mathrm{NMR}\left(282 \mathrm{MHz}, \mathrm{CDCl}_{3}, \delta\right):-62.12(\mathrm{t}, J=10.0 \mathrm{~Hz}),-103.04$ (tt, $\left.J=8.3,5.3 \mathrm{~Hz}\right)$.

1-(4-Bromophenyl)-3,3,3-trifluoropropan-1-one, $2 \mathrm{f}^{3}$<smiles>O=C(CC(F)(F)F)c1ccc(Br)cc1</smiles>

Yield $72 \%$ (192.3 mg, $0.72 \mathrm{mmol}$ ). White crystals, $\mathrm{mp}=71-72{ }^{\circ} \mathrm{C}$ (lit. $70-71{ }^{\circ} \mathrm{C}^{3}$ ). $\mathrm{R}_{f}=0.73$ (PE:EtOAc $=5: 1)$.

${ }^{1} \mathrm{H}$ NMR (300 MHz, $\left.\mathrm{CDCl}_{3}, \delta\right): 7.78(\mathrm{~d}, J=8.5 \mathrm{~Hz}, 2 \mathrm{H}), 7.63(\mathrm{~d}, J=8.5 \mathrm{~Hz}, 2 \mathrm{H}), 3.76(\mathrm{q}, J=9.9$ $\mathrm{Hz}, 2 \mathrm{H})$.

${ }^{13} \mathrm{C}$ NMR $\left(75 \mathrm{MHz}, \mathrm{CDCl}_{3}, \delta\right): 188.7$ (q, $\left.{ }^{3} J_{\mathrm{CF}}=2.2 \mathrm{~Hz}\right), 134.7$ (q, $\left.{ }^{4} J_{\mathrm{CF}}=1.5 \mathrm{~Hz}\right), 132.4,129.9$, 129.8, $124.0\left(\mathrm{q},{ }^{1} \mathrm{~J}_{\mathrm{CF}}=277.0 \mathrm{~Hz}\right), 42.2\left(\mathrm{q},{ }^{2} J_{\mathrm{CF}}=28.5 \mathrm{~Hz}\right)$.

${ }^{19} \mathrm{~F} \mathrm{NMR}\left(282 \mathrm{MHz} \mathrm{CDCl}_{3}, \delta\right):-62.81$ (t, $\left.J=9.9 \mathrm{~Hz}\right)$.

2-(Trifluoromethyl)-2,3-dihydro-1H-inden-1-one, $2 \mathrm{~g}^{13}$<smiles>O=C1c2ccccc2CC1C(F)(F)F</smiles>

Yield 81\% (162.2 mg, $0.81 \mathrm{mmol})$. Colorless oil. $\mathrm{R}_{\mathrm{f}}=0.73(\mathrm{PE}: \mathrm{EtOAc}=10: 1)$.

${ }^{1} \mathrm{H}$ NMR (300 MHz, $\left.\mathrm{CDCl}_{3}, \delta\right): 7.80(\mathrm{~d}, J=7.5 \mathrm{~Hz}, 1 \mathrm{H}), 7.66(\mathrm{t}, J=7.5 \mathrm{~Hz}, 1 \mathrm{H}), 7.52(\mathrm{~d}, J=7.5$ $\mathrm{Hz}, 1 \mathrm{H}), 7.42(\mathrm{t}, J=7.5 \mathrm{~Hz}, 1 \mathrm{H}), 3.50-3.26(\mathrm{~m}, 3 \mathrm{H})$.

${ }^{13} \mathrm{C} \mathrm{NMR}\left(75 \mathrm{MHz}_{\mathrm{CDCl}}, \mathrm{\delta}\right): 197.0$ (q, $\left.{ }^{3} \mathrm{~J}_{\mathrm{CF}}=2.0 \mathrm{~Hz}\right), 152.2,136.0,128.3,126.7,125.1$ (q, ${ }^{1} \mathrm{~J}_{\mathrm{CF}}$ $=278.5 \mathrm{~Hz}$ ), 123.2, $49.9\left(\mathrm{q},{ }^{2} J_{\mathrm{CF}}=27.4 \mathrm{~Hz}\right), 27.7$ (q, $\left.{ }^{3} J_{\mathrm{CF}}=2.5 \mathrm{~Hz}\right)$.

${ }^{19} \mathrm{~F} \mathrm{NMR}\left(282 \mathrm{MHz}, \mathrm{CDCl}_{3}, \delta\right):-68.55(\mathrm{~d}, J=8.2 \mathrm{~Hz})$.

2-(Trifluoromethyl)-3,4-dihydronaphthalen-1(2H)-one, $2 \mathrm{~h}{ }^{17}$<smiles>O=C1c2ccccc2CCC1C(F)(F)F</smiles>

Yield 69\% (147.8 mg, $0.69 \mathrm{mmol})$. Colorless oil. $\mathrm{R}_{\mathrm{f}}=0.77(\mathrm{PE}: \mathrm{EtOAc}=10: 1)$. 
${ }^{1} \mathrm{H}$ NMR $\left(300 \mathrm{MHz}, \mathrm{CDCl}_{3}, \delta\right): 8.03(\mathrm{dd}, J=7.7,1.5 \mathrm{~Hz}, 1 \mathrm{H}), 7.51$ (td, $\left.J=7.7,1.5 \mathrm{~Hz}, 1 \mathrm{H}\right), 7.40$ $-7.21(\mathrm{~m}, 2 \mathrm{H}$ ), 3.25 (ddq, $J=16.2,8.8,4.4 \mathrm{~Hz}, 1 \mathrm{H}), 3.09$ (dd, $J=9.9,4.8 \mathrm{~Hz}, 2 \mathrm{H}$ ), 2.47 (dq, $J=$ 13.6, $4.4 \mathrm{~Hz}, 1 \mathrm{H}), 2.32-2.16(\mathrm{~m}, 1 \mathrm{H})$.

${ }^{13} \mathrm{C} \mathrm{NMR}\left(75 \mathrm{MHz}, \mathrm{CDCl}_{3}, \delta\right): 190.3,143.2,134.3,132.0,128.9,127.9,127.2,125.2$ (q, ${ }^{1} \mathrm{JCF}=$ $279.8 \mathrm{~Hz}), 51.0\left(\mathrm{q},{ }^{2} \mathrm{~J}_{\mathrm{CF}}=25.6 \mathrm{~Hz}\right), 27.6,23.5\left(\mathrm{q},{ }^{3} \mathrm{~J}_{\mathrm{CF}}=2.6 \mathrm{~Hz}\right)$.

${ }^{19} \mathrm{~F} \mathrm{NMR}\left(282 \mathrm{MHz}, \mathrm{CDCl}_{3}, \delta\right):-67.57(\mathrm{~d}, J=8.8 \mathrm{~Hz}, 3 \mathrm{~F})$.

\section{3,3,3-Trifluoro-2-methyl-1-phenylpropan-1-one, $2 i^{3}$}<smiles>CC(C(=O)c1ccccc1)C(F)(F)F</smiles>

Yield 67\% (135.5 mg, $0.67 \mathrm{mmol})$. Colorless oil. $\mathrm{R}_{\mathrm{f}}=0.80(\mathrm{PE}: \mathrm{EtOAC}=10: 1)$.

${ }^{1} \mathrm{H}$ NMR (300 MHz, CDCl $\left.3, \delta\right): 7.95$ (d, $J=7.5 \mathrm{~Hz}, 2 \mathrm{H}$ ), 7.62 (t, $\left.J=7.5 \mathrm{~Hz}, 1 \mathrm{H}\right), 7.50$ (t, $J=7.5$ $\mathrm{Hz}, 2 \mathrm{H}$ ), 4.27 (sept, $J=7.4 \mathrm{~Hz}, 1 \mathrm{H}$ ), 1.47 (d, $J=7.4 \mathrm{~Hz}, 3 \mathrm{H}$ ).

${ }^{13} \mathrm{C}$ NMR $\left(75 \mathrm{MHz}, \mathrm{CDCl}_{3}, \delta\right): 194.6\left(\mathrm{q},{ }^{3} \mathrm{JCF}_{\mathrm{CF}} 2.0 \mathrm{~Hz}\right), 135.8,134.1,129.0,128.7,125.5$ (q, ${ }^{1} \mathrm{~J}_{\mathrm{CF}}$ $=280.1 \mathrm{~Hz}), 44.4\left(\mathrm{q},{ }^{2} \mathrm{~J}_{\mathrm{CF}}=26.5 \mathrm{~Hz}\right), 11.8\left(\mathrm{q},{ }^{3} \mathrm{~J}_{\mathrm{CF}}=2.7 \mathrm{~Hz}\right)$.

${ }^{19} \mathrm{~F} \mathrm{NMR}\left(282 \mathrm{MHz}, \mathrm{CDCl}_{3}, \delta\right):-69.10(\mathrm{~d}, J=7.8 \mathrm{~Hz}, 3 \mathrm{~F})$.

\section{3,3,3-Trifluoro-1-(naphthalen-2-yl)propan-1-one, $2 \mathrm{j}^{13}$}<smiles>O=C(CC(F)(F)F)c1ccc2ccccc2c1</smiles>

Yield $20 \%$ (47.6 mg, $0.20 \mathrm{mmol}$ ). White crystals, $\mathrm{mp}=73-74{ }^{\circ} \mathrm{C}$ (lit. $\left.74{ }^{\circ} \mathrm{C}^{13}\right), \mathrm{Rf}=0.85$ (PE:EtOAc $=10: 1)$.

${ }^{1} \mathrm{H}$ NMR (300 MHz, $\left.\mathrm{CDCl}_{3}, \delta\right): 8.39(\mathrm{~s}, 1 \mathrm{H}), 8.01-7.86(\mathrm{~m}, 4 \mathrm{H}), 7.67-7.55(\mathrm{~m}, 2 \mathrm{H}), 3.92(\mathrm{q}, J=$ $10.0 \mathrm{~Hz}, 2 \mathrm{H})$.

${ }^{13} \mathrm{C} \mathrm{NMR}\left(75 \mathrm{MHz}, \mathrm{CDCl}_{3}, \delta\right): 189.8\left(\mathrm{q},{ }^{3} \mathrm{~J}_{\mathrm{CF}}=2.4 \mathrm{~Hz}\right), 136.1,133.3,132.4,130.6,129.8,129.3$, $129.0,128.0,127.3,124.3\left(q,{ }^{1} J_{C F}=277.0 \mathrm{~Hz}\right), 123.5,42.2\left(q,{ }^{2} J_{C F}=28.2 \mathrm{~Hz}\right)$.

${ }^{19} \mathrm{~F} \mathrm{NMR}\left(282 \mathrm{MHz}, \mathrm{CDCl}_{3}, \delta\right):-62.67(\mathrm{t}, J=10.0 \mathrm{~Hz}, 3 \mathrm{~F})$.

\section{2-(Trifluoromethyl)cyclopentan-1-one, $2 \mathrm{k}^{17}$}

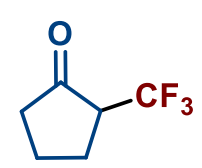

Yield 66\% (100.4 mg, $0.66 \mathrm{mmol}$ ). Colorless oil. $\mathrm{R}_{\mathrm{f}}=0.91$ (PE:EtOAc $\left.=10: 1\right)$.

${ }^{1} \mathrm{H}$ NMR (300 MHz, CDCl $\left.3, \delta\right): 2.85$ (sext, $\left.J=9.6 \mathrm{~Hz}, 1 \mathrm{H}\right), 2.39-1.99(\mathrm{~m}, 5 \mathrm{H}), 1.92-1.78(\mathrm{~m}$, $1 \mathrm{H})$.

${ }^{13} \mathrm{C} \mathrm{NMR}\left(75 \mathrm{MHz}, \mathrm{CDCl}_{3}, \delta\right): 209.6,124.8\left(\mathrm{q},{ }^{1} \mathrm{JCF}=277.9 \mathrm{~Hz}\right), 51.2\left(\mathrm{q},{ }^{2} \mathrm{JCF}=27.3 \mathrm{~Hz}\right), 38.5(\mathrm{q}$, $\left.{ }^{4} J_{\mathrm{CF}}=1.6 \mathrm{~Hz}\right), 24.4\left(\mathrm{q},{ }^{3} \mathrm{~J}_{\mathrm{CF}}=1.8 \mathrm{~Hz}\right), 20.1$.

${ }^{19} \mathrm{~F}$ NMR $\left(282 \mathrm{MHz}, \mathrm{CDCl}_{3}, \delta\right):-68.33(\mathrm{~d}, J=9.3 \mathrm{~Hz}, 3 \mathrm{~F})$. 
<smiles>O=C1CCCCC1C(F)(F)F</smiles>

Yield 75\% (124.6 mg, $0.75 \mathrm{mmol})$. Colorless oil. $\mathrm{R}_{\mathrm{f}}=0.84(\mathrm{PE}: \mathrm{EtOAc}=10: 1)$.

${ }^{1} \mathrm{H}$ NMR (300 MHz, $\left.\mathrm{CDCl}_{3}, \delta\right): 3.16-2.96(\mathrm{~m}, 1 \mathrm{H}), 2.50-2.25(\mathrm{~m}, 3 \mathrm{H}), 2.12-1.93(\mathrm{~m}, 2 \mathrm{H}), 1.83$ $-1.66(\mathrm{~m}, 3 \mathrm{H})$.

${ }^{13} \mathrm{C} \mathrm{NMR}\left(75 \mathrm{MHz}, \mathrm{CDCl}_{3}, \delta\right): 203.1,124.8$ (q, $\left.{ }^{1} \mathrm{~J}_{\mathrm{CF}}=279.3 \mathrm{~Hz}\right), 53.7$ (q, $\left.{ }^{2} \mathrm{~J}_{\mathrm{CF}}=25.4 \mathrm{~Hz}\right), 42.3$ (q, $\left.{ }^{4} J_{C F}=1.3 \mathrm{~Hz}\right), 27.7\left(\mathrm{q},{ }^{3} J_{\mathrm{CF}}=2.3 \mathrm{~Hz}\right), 27.2,23.9$.

${ }^{19} \mathrm{~F} \mathrm{NMR}\left(282 \mathrm{MHz} \mathrm{CDCl}_{3}, \delta\right):-69.73$ (d, J=7.8 Hz, 3F).

4-(Tert-butyl)-2-(trifluoromethyl)cyclohexan-1-one, $2 \mathrm{~m}^{17}$<smiles>CC(C)(C)C1CCC(=O)C(C(F)(F)F)C1</smiles>

Yield $78 \%$ (173.4 $\mathrm{mg}, 0.78 \mathrm{mmol})$. The product is formed as a mixture 0.4:0.6 of trans- and cisdiastereomers. Colorless oil. $R_{f}=0.69(P E: E t O A c=10: 1)$.

${ }^{1} \mathrm{H}$ NMR (300 MHz, $\left.\mathrm{CDCl}_{3}, \delta\right): 3.19-2.99(\mathrm{~m}, 1 \mathrm{H}), 2.51-2.00(\mathrm{~m}, 4 \mathrm{H}), 1.85-1.38(\mathrm{~m}, 3 \mathrm{H}), 0.93$ $(\mathrm{s}, 5.4 \mathrm{H}), 0.91(\mathrm{~s}, 3.6 \mathrm{H})$.

${ }^{13} \mathrm{C}$ NMR (75 MHz, $\left.\mathrm{CDCl}_{3}, \delta\right)$ : trans-diastereomer: 204.9, 125.2 (q, ${ }^{1} \mathrm{~J}_{\mathrm{CF}}=281.0 \mathrm{~Hz}$ ), 52.3 (q, ${ }^{2} \mathrm{~J}_{\mathrm{CF}}$ $=25.5 \mathrm{~Hz}$ ), 41.8, $40.5\left(\mathrm{q},{ }^{4} \mathrm{~J}_{\mathrm{CF}}=1.6 \mathrm{~Hz}\right), 32.6,27.3,26.5\left(\mathrm{q},{ }^{3} \mathrm{~J}_{\mathrm{CF}}=2.0 \mathrm{~Hz}\right), 26.2$. cis-diastereomer: 203.3, 124.8 (q, $\left.{ }^{1} J_{\mathrm{CF}}=279.2 \mathrm{~Hz}\right), 53.1\left(\mathrm{q},{ }^{2} J_{\mathrm{CF}}=25.2 \mathrm{~Hz}\right), 46.2,41.8,32.7,28.7\left(\mathrm{q},{ }^{3} \mathrm{~J}_{\mathrm{CF}}=2.2\right.$ $\mathrm{Hz})$, 28.2, 27.6.

${ }^{19} \mathrm{~F} \mathrm{NMR}\left(282 \mathrm{MHz}, \mathrm{CDCl}_{3}, \delta\right):-66.34$ (d, J=10.2 Hz, 3F, cis), -69.98 (d, J=7.9 Hz, 3F, trans).

2-(Trifluoromethyl)cycloheptan-1-one, 2n ${ }^{17}$<smiles>O=C1CCCCCC1C(F)(F)F</smiles>

Yield 69\% (124.3 mg, $0.69 \mathrm{mmol})$. Colorless oil. $\mathrm{R}_{\mathrm{f}}=0.74$ (PE:EtOAc = 10:1).

${ }^{1} \mathrm{H}$ NMR (300 MHz, $\left.\mathrm{CDCl}_{3}, \delta\right): 3.29-3.14(\mathrm{~m}, 1 \mathrm{H}), 2.59-2.49(\mathrm{~m}, 2 \mathrm{H}), 2.17-2.04(\mathrm{~m}, 1 \mathrm{H}), 2.00$ $-1.82(\mathrm{~m}, 3 \mathrm{H}), 1.69-1.27(\mathrm{~m}, 4 \mathrm{H})$.

${ }^{13} \mathrm{C} \mathrm{NMR}\left(75 \mathrm{MHz}, \mathrm{CDCl}_{3}, \delta\right): 206.1,125.1$ (q, $\left.{ }^{1} \mathrm{~J}_{\mathrm{CF}}=279.9 \mathrm{~Hz}\right), 55.5\left(\mathrm{q},{ }^{2} \mathrm{~J}_{\mathrm{CF}}=24.8 \mathrm{~Hz}\right), 43.2(\mathrm{q}$, $\left.{ }^{4} J_{\mathrm{CF}}=1.6 \mathrm{~Hz}\right), 29.1,27.6,24.8\left(\mathrm{q},{ }^{3} \mathrm{~J}_{\mathrm{CF}}=2.2 \mathrm{~Hz}\right), 24.4$.

${ }^{19} \mathrm{~F} \mathrm{NMR}\left(282 \mathrm{MHz}_{\mathrm{CDCl}}\right.$, $\left.\delta\right):-69.34$ (d, J=8.7 Hz, 3F).

\section{2-(Trifluoromethyl)cyclododecan-1-one, 20}




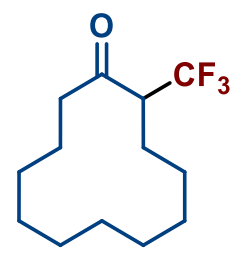

Yield 71\% (177.7 mg, $0.71 \mathrm{mmol})$. Colorless oil. $\mathrm{R}_{\mathrm{f}}=0.82$ (PE:EtOAc $\left.=10: 1\right)$.

${ }^{1} \mathrm{H}$ NMR $\left(300 \mathrm{MHz}, \mathrm{CDCl}_{3}, \delta\right): 3.26$ (ddq, $J=14.7,8.8,2.9 \mathrm{~Hz}, 1 \mathrm{H}$ ), 2.71 (ddd, $J=16.9,11.0,3.7$ $\mathrm{Hz}, 1 \mathrm{H}), 2.51$ (ddd, $J=16.9,7.3,3.7 \mathrm{~Hz}, 1 \mathrm{H}), 2.12-1.96(\mathrm{~m}, 1 \mathrm{H}), 1.92-1.78(\mathrm{~m}, 1 \mathrm{H}), 1.74-$ $1.51(\mathrm{~m}, 2 \mathrm{H}), 1.46-1.13(\mathrm{~m}, 14 \mathrm{H})$.

${ }^{13} \mathrm{C} \mathrm{NMR}\left(75 \mathrm{MHz}, \mathrm{CDCl}_{3}, \delta\right): 204.9,125.2\left(\mathrm{q},{ }^{1} \mathrm{~J}_{\mathrm{CF}}=280.3 \mathrm{~Hz}\right), 55.6$ (q, $\left.{ }^{2} \mathrm{~J}_{\mathrm{CF}}=25.5 \mathrm{~Hz}\right), 38.9$, 25.8, 25.5, 24.2, 24.20, 24.16, 24.1, 22.8, 22.4, 21.9.

${ }^{19} \mathrm{~F}$ NMR $\left(282 \mathrm{MHz}, \mathrm{CDCl}_{3}, \delta\right):-67.93(\mathrm{~d}, J=9.0 \mathrm{~Hz}, 3 \mathrm{~F})$.

HRMS (ESI-TOF) $\mathrm{m} / \mathrm{z}[\mathrm{M}+\mathrm{Na}]^{+}$. Calcd for $\left[\mathrm{C}_{13} \mathrm{H}_{21} \mathrm{~F}_{3} \mathrm{ONa}\right]^{+}:$273.1437. Found: 273.1442.

Anal. Calcd for $\mathrm{C}_{13} \mathrm{H}_{21} \mathrm{~F}_{3} \mathrm{O}$ : C, 62.38; F, 22.77; $\mathrm{H}, 8.46$. Found: $\mathrm{C}, 62.27 ; \mathrm{F}, 22.85 ; \mathrm{H}, 8.26$.

IR (thin layer): 2394, 2866, 1756, 1722, 1470, 1445, 1260, 1201, 1164, $1110 \mathrm{~cm}^{-1}$.

\section{General Experimental Procedure for Scheme 3}

An undivided cell was equipped with a platinum plate anode $\left(3 \mathrm{~cm}^{2}\right)$ and a platinum plate cathode $\left(3 \mathrm{~cm}^{2}\right)$ and connected to a DC regulated power supply. The solution of enol acetate $1(1.0 \mathrm{mmol})$, $\mathrm{C}_{4} \mathrm{~F}_{9} \mathrm{SO}_{2} \mathrm{Na}(2.0 \mathrm{mmol}, 612.2 \mathrm{mg})$ and $n-\mathrm{Bu}_{4} \mathrm{NBF}_{4}(0.5 \mathrm{mmol}, 164.6 \mathrm{mg})$ in $10 \mathrm{~mL}$ of $\mathrm{CH}_{3} \mathrm{CN} / \mathrm{H}_{2} \mathrm{O}$ (8:2) was electrolyzed using constant current conditions $\left(\mathrm{j}=6.7 \mathrm{~mA} / \mathrm{cm}^{2}\right)$ at $20-25{ }^{\circ} \mathrm{C}$ under magnetic stirring for 241 min with I $=20 \mathrm{~mA}$. Then electrodes were washed with $\mathrm{CH}_{2} \mathrm{Cl}_{2}(2 \times 20$ $\mathrm{mL})$. The combined organic phases were washed with brine $(3 \times 10 \mathrm{~mL})$, dried over $\mathrm{MgSO}_{4}$, filtered and concentrated under reduced pressure using a rotary evaporator $(15-20 \mathrm{mmHg}$ ), (bath temperature, ca. $\left.20-25{ }^{\circ} \mathrm{C}\right)$. Product 3 was isolated by chromatography on $\mathrm{SiO}_{2}(\mathrm{PE}: \mathrm{EtOAc}=$ $10: 1+2 \% \mathrm{HCOOH})$.

\section{2-(Perfluorobutyl)-1-(4-methoxyphenyl)ethan-1-one, 3c}<smiles>COc1ccc(C(=O)CC(F)(F)F)cc1</smiles>

Yield 43\% (158.3 mg, $0.43 \mathrm{mmol}$ ). Colorless oil. $\mathrm{R}_{\mathrm{f}}=0.87$ (PE:EtOAc $\left.=10: 1\right)$.

${ }^{1} \mathrm{H}$ NMR $\left(300 \mathrm{MHz}, \mathrm{CDCl}_{3}, \delta\right): 7.91(\mathrm{~d}, J=8.9 \mathrm{~Hz}, 2 \mathrm{H}), 6.96(\mathrm{~d}, J=8.9 \mathrm{~Hz}, 2 \mathrm{H}), 3.88(\mathrm{~s}, 3 \mathrm{H})$, $3.66(\mathrm{t}, J=18.0 \mathrm{~Hz}, 2 \mathrm{H})$.

${ }^{13} \mathrm{C}$ NMR $\left(75 \mathrm{MHz}, \mathrm{CDCl}_{3}, \delta\right): 188.1,164.6,131.2,129.7,117.5$ (qt, ${ }^{1} \mathrm{JCF}=287.7,{ }^{2} \mathrm{JCF}=33.2 \mathrm{~Hz}$, $\left.\mathrm{CF}_{3}\right), 116.5\left(\mathrm{tt},{ }^{1} \mathrm{JCF}_{\mathrm{CF}} 259.4,{ }^{2} \mathrm{JCF}_{\mathrm{CF}}=32.7 \mathrm{~Hz}, \mathrm{CF}_{2} \mathrm{CH}_{2}\right), 114.2,55.6,38.4\left(\mathrm{t},{ }^{2} \mathrm{JCF}=21.5 \mathrm{~Hz}\right) ; \mathrm{CF}_{2} \mathrm{CF}_{2}$ not resolved. 
${ }^{19} \mathrm{~F} \mathrm{NMR}\left(282 \mathrm{MHz} \mathrm{CDCl}_{3}, \delta\right):-82.76(\mathrm{brt}, \mathrm{J} \approx 9.1 \mathrm{~Hz}, 3 \mathrm{~F}),-112.80\left(\mathrm{tt},{ }^{3} J_{\mathrm{FH}}=18.0,{ }^{4} \mathrm{JFF}_{\mathrm{F}}=14.8\right.$ $\mathrm{Hz}, 2 \mathrm{~F}),-125.50--125.25(\mathrm{~m}, 2 \mathrm{~F}),-127.67--127.44(\mathrm{~m}, 2 \mathrm{~F})$.

HRMS (ESI-TOF) m/z [M+H] $]^{+}$. Calcd for $\left[\mathrm{C}_{13} \mathrm{H}_{10} \mathrm{~F}_{9} \mathrm{O}\right]^{+}:$369.0532. Found: 369.0525.

Anal. Calcd for $\mathrm{C}_{13} \mathrm{H}_{9} \mathrm{~F}_{9} \mathrm{O}_{2}$ : C, 42.41; F, 46.44; $\mathrm{H}, 2.46$. Found: C, 42.33; F, 46.30; H, 2.52.

IR (thin layer): 1687, 1603, 1577, 1513, 1351, 1233, 1176, 1134, 1030, $858 \mathrm{~cm}^{-1}$.

\section{2-(Perfluorobutyl)-1-(4-chlorophenyl)ethan-1-one, 3d}<smiles>O=C(CC(F)(F)F)c1ccc(Cl)cc1</smiles>

Yield 58\% (216.1 mg, $0.58 \mathrm{mmol})$. White crystals, $\mathrm{mp}=44-45^{\circ} \mathrm{C} \mathrm{R}_{\mathrm{f}}=0.75(\mathrm{PE}: \mathrm{EtOAc}=10: 1)$.

${ }^{1} \mathrm{H}$ NMR (300 MHz, $\left.\mathrm{CDCl}_{3}, \delta\right): 7.87(\mathrm{~d}, J=8.6 \mathrm{~Hz}, 2 \mathrm{H}), 7.48(\mathrm{~d}, J=8.5 \mathrm{~Hz}, 2 \mathrm{H}), 3.70(\mathrm{t}, J=17.7$ $\mathrm{Hz}, 2 \mathrm{H})$.

${ }^{13} \mathrm{C}$ NMR $\left(75 \mathrm{MHz} \mathrm{CDCl}_{3}, \delta\right): 188.6,141.1,134.8,130.1,129.5,117.5$ (qt, ${ }^{1} \mathrm{JCF}_{\mathrm{CF}}=288.3,{ }^{2} \mathrm{~J} \mathrm{CF}=$ 33. $\left.2 \mathrm{~Hz}, \mathrm{CF}_{3}\right), 116.3\left(\mathrm{tt},{ }^{1} \mathrm{~J}_{\mathrm{CF}}=259.4,{ }^{2} \mathrm{~J}_{\mathrm{CF}}=32.7 \mathrm{~Hz}, \mathrm{CF}_{2} \mathrm{CH}_{2}\right), 38.8\left(\mathrm{t},{ }^{2} \mathrm{~J}_{\mathrm{CF}}=21.5 \mathrm{~Hz}\right) ; \mathrm{CF}_{2} \mathrm{CF}_{2}$ not resolved.

${ }^{19} \mathrm{~F}$ NMR (282 MHz, $\left.\mathrm{CDCl}_{3}, \delta\right):-81.03$ - -81.34 (m, 3F), -110.99 - -111.37 (m, 2F), -123.57 - $123.86(\mathrm{~m}, 2 \mathrm{~F}),-125.78--126.15(\mathrm{~m}, 2 \mathrm{~F})$.

HRMS (ESI-TOF) m/z [M+Na] $]^{+}$. Calcd for $\left[\mathrm{C}_{12} \mathrm{H}_{6} \mathrm{ClF}_{9} \mathrm{ONa}\right]^{+}:$394.9856. Found: 394.9852.

Anal. Calcd for $\mathrm{C}_{12} \mathrm{H}_{6} \mathrm{ClF}_{9} \mathrm{O}$ : C, 38.68; F, 45.89; Cl, 9.51; H, 1.62. Found: C, 38.73; F, 45.66; Cl, $9.74 ; \mathrm{H}, 1.49$.

IR (KBr): 3102, 3044, 2944, 1695, 1592, 1354, 1235, 1134, 997, 866, 825, 740, $530 \mathrm{~cm}^{-1}$.

\section{2-(Perfluorobutyl)-3,4-dihydronaphthalen-1(2H)-one, $3 \mathrm{~h}^{18}$}<smiles>O=C1c2ccccc2CCC1C(F)(F)F</smiles>

Yield 32\% (116.5 mg, $0.32 \mathrm{mmol})$. White crystals, $\mathrm{mp}=45-46{ }^{\circ} \mathrm{C} \mathrm{R}_{\mathrm{f}}=0.81$ (PE:EtOAc $\left.=10: 1\right)$. ${ }^{1} \mathrm{H}$ NMR $\left(300 \mathrm{MHz}, \mathrm{CDCl}_{3}, \delta\right): 8.02(\mathrm{~d}, J=7.6 \mathrm{~Hz}, 1 \mathrm{H}), 7.50(\mathrm{t}, J=7.6 \mathrm{~Hz}, 1 \mathrm{H}), 7.31$ (t, $J=7.6$ $\mathrm{Hz}, 1 \mathrm{H}), 7.25$ (d, J=7.6 Hz, 1H), $3.51-3.30(\mathrm{~m}, 1 \mathrm{H}), 3.19$ (ddd, J=16.9, 5.9, $5.9 \mathrm{~Hz}, 1 \mathrm{H}), 3.02$ ddd, $J=16.9,8.1,5.1 \mathrm{~Hz}, 1 \mathrm{H}), 2.55-2.31(\mathrm{~m}, 2 \mathrm{H})$.

${ }^{13} \mathrm{C}$ NMR $\left(75 \mathrm{MHz}, \mathrm{CDCl}_{3}, \delta\right): 190.4\left(\mathrm{t},{ }^{3} \mathrm{~J} \mathrm{CF}=2.7 \mathrm{~Hz}\right), 143.1,134.3,132.4,128.9,128.0,127.2$, $117.58\left(\mathrm{qt},{ }^{1} \mathrm{~J}_{\mathrm{CF}}=288.8,{ }^{2} \mathrm{~J}_{\mathrm{CF}}=33.2 \mathrm{~Hz}, \mathrm{CF}_{3}\right), 117.62\left(\mathrm{tt},{ }^{1} \mathrm{~J}_{\mathrm{CF}}=260.5,{ }^{2} \mathrm{~J}_{\mathrm{CF}}=32.7 \mathrm{~Hz}, C \mathrm{~F}_{2} \mathrm{CH}\right)$, $49.5\left(\mathrm{t},{ }^{2} \mathrm{~J}_{\mathrm{CF}}=20.2 \mathrm{~Hz}\right), 27.3,23.2-23.0(\mathrm{~m}) ; \mathrm{CF}_{2} \mathrm{CF}_{2}$ not resolved.

${ }^{19} \mathrm{~F}$ NMR $\left(282 \mathrm{MHz} \mathrm{CDCl}_{3}, \delta\right):-81.73\left(\mathrm{t},{ }^{4} J_{\mathrm{FF}}=9.6 \mathrm{~Hz}, 3 \mathrm{~F}\right),-110.69\left(\mathrm{dm}, J_{\mathrm{AB}}=284.0 \mathrm{~Hz}, \mathrm{~F}_{\mathrm{A}}\right)$, $113.31\left(\mathrm{dm}, J_{A B}=284.0 \mathrm{~Hz}, F_{B}\right),-120.24\left(\mathrm{dm}, J_{A B}=294.6 \mathrm{~Hz}, F_{A}\right),-122.24\left(\mathrm{dm}, J_{A B}=294.6 \mathrm{~Hz}\right.$, $\left.F_{B}\right),-126.90\left(t,{ }^{4} J_{F F}=14.8 \mathrm{~Hz}, 2 F\right)$.

HRMS (ESI-TOF) m/z [M+H] $]^{+}$. Calcd for $\left[\mathrm{C}_{14} \mathrm{H}_{10} \mathrm{~F}_{9} \mathrm{O}\right]^{+}:$365.0582. Found: 365.0582. 


\section{2-(Perfluorobutyl)-1-(phenyl)propan-1-one, 3i}<smiles>CC(C(=O)c1ccccc1)C(F)(F)F</smiles>

Yield 40\% (140.9 mg, $0.40 \mathrm{mmol})$. Colorless oil. $\mathrm{R}_{\mathrm{f}}=0.89(\mathrm{PE}: \mathrm{EtOAc}=10: 1)$.

${ }^{1} \mathrm{H}$ NMR $\left(300 \mathrm{MHz}, \mathrm{CDCl}_{3}, \delta\right): 7.94(\mathrm{~d}, J=7.8 \mathrm{~Hz}, 2 \mathrm{H}), 7.63(\mathrm{t}, J=7.3 \mathrm{~Hz}, 1 \mathrm{H}), 7.51$ (t, $J=7.3$ $\mathrm{Hz}, 2 \mathrm{H}), 4.39$ (tq, $J=13.9,7.3 \mathrm{~Hz}, 1 \mathrm{H}), 1.49$ (d, $J=7.3 \mathrm{~Hz}, 3 \mathrm{H})$.

${ }^{13} \mathrm{C} \mathrm{NMR}\left(75 \mathrm{MHz}, \mathrm{CDCl}_{3}, \delta\right): 194.7\left(\mathrm{t},{ }^{3} \mathrm{~J} \mathrm{CF}=2.7 \mathrm{~Hz}\right), 136.2\left(\mathrm{t},{ }^{4} \mathrm{~J}_{\mathrm{CF}}=1.6 \mathrm{~Hz}\right), 134.2,129.1,128.6$, 117.5 (qt, $\left.{ }^{1} J_{C F}=287.7,{ }^{2} J_{C F}=33.2 \mathrm{~Hz}, C F_{3}\right), 117.2\left(\mathrm{tt},{ }^{1} J_{C F}=260.5,{ }^{2} J_{C F}=32.2 \mathrm{~Hz}, C F_{2} \mathrm{CH}\right), 41.1$ $\left(\mathrm{t},{ }^{2} \mathrm{~J}_{\mathrm{CF}}=20.7 \mathrm{~Hz}\right), 11.6-11.5(\mathrm{~m}) ; \mathrm{CF}_{2} \mathrm{CF}_{2}$ not resolved.

${ }^{19} \mathrm{~F} \mathrm{NMR}\left(282 \mathrm{MHz}, \mathrm{CDCl}_{3}, \delta\right):-81.89\left(\mathrm{t},{ }^{4} \mathrm{JFF}=9.6 \mathrm{~Hz}, 3 \mathrm{~F}\right),-115.16(\mathrm{q}, \mathrm{J} \approx 14.8 \mathrm{~Hz}, 2 \mathrm{~F}),-121.75$ $\left(\mathrm{dm}, J_{\mathrm{AB}}=294.5 \mathrm{~Hz}, \mathrm{~F}_{\mathrm{A}}\right),-122.39\left(\mathrm{dm}, J_{\mathrm{AB}}=294.5 \mathrm{~Hz}, \mathrm{~F}_{\mathrm{B}}\right),-126.58\left(\mathrm{dt}, J_{\mathrm{AB}}=292.5 \mathrm{~Hz},{ }^{4} J_{\mathrm{FF}}=\right.$ $\left.14.8 \mathrm{~Hz}, \mathrm{~F}_{\mathrm{A}}\right),-127.12\left(\mathrm{dt}, J_{\mathrm{AB}}=292.5,{ }^{4} J_{\mathrm{FF}}=14.8 \mathrm{~Hz}, \mathrm{~F}_{\mathrm{B}}\right)$.

HRMS (ESI-TOF) m/z [M+H] $]^{+}$. Calcd for $\left[\mathrm{C}_{13} \mathrm{H}_{10} \mathrm{~F}_{9} \mathrm{O}^{+}:\right.$: 353.0582. Found: 353.0584 .

Anal. Calcd for $\mathrm{C}_{13} \mathrm{H}_{9} \mathrm{~F}_{9} \mathrm{O}$ : C, 44.33; F, 48.55; H, 2.58. Found: C, 44.17; F, 48.30; H, 2.43.

IR (thin layer): 3069, 3001, 1696, 1462, 1351, 1230, 1136, 1021, 962, 850, 752, 705, $690 \mathrm{~cm}^{-1}$.

\section{Experimental procedure for Scheme 4a.}

An undivided cell was equipped with a platinum plate anode $\left(3 \mathrm{~cm}^{2}\right)$ and a platinum plate cathode $\left(3 \mathrm{~cm}^{2}\right)$ and connected to a DC regulated power supply. The solution of enol acetate $1 \mathrm{a}(1.0 \mathrm{mmol}$, $162.0 \mathrm{mg})$ and $n-\mathrm{Bu}_{4} \mathrm{NBF}_{4}(0.5 \mathrm{mmol}, 164.6 \mathrm{mg})$ in $10 \mathrm{~mL}$ of $\mathrm{CH}_{3} \mathrm{CN} / \mathrm{H}_{2} \mathrm{O}$ (8:2) was electrolyzed using constant current conditions $\left(\mathrm{j}=6.7 \mathrm{~mA} / \mathrm{cm}^{2}\right)$ at $20-25^{\circ} \mathrm{C}$ under magnetic stirring for $241 \mathrm{~min}$ with I = $20 \mathrm{~mA}$. Then electrodes were washed with $\mathrm{CH}_{2} \mathrm{Cl}_{2}(2 \times 20 \mathrm{~mL})$. The combined organic phases were washed with brine $(3 \times 10 \mathrm{~mL})$, dried over $\mathrm{MgSO}_{4}$, filtered and concentrated under reduced pressure using a rotary evaporator $(15-20 \mathrm{mmHg})$, (bath temperature, ca. $20-25^{\circ} \mathrm{C}$ ). Products $4 \mathrm{a}(23 \%, 41.0 \mathrm{mg}, 0.23 \mathrm{mmol})$ and $5 \mathrm{a}(31 \%, 42.0 \mathrm{mg}, 0.31 \mathrm{mmol})$ were isolated by chromatography on $\mathrm{SiO}_{2}(\mathrm{PE}:$ EtOAc $=$ from 20:1 to 2:1).

\section{2-Acetoxyacetophenone, $4 a^{19}$}<smiles>CC(=O)OCC(=O)c1ccccc1</smiles>

Yellow crystals, $\mathrm{mp}=41^{\circ} \mathrm{C}\left(\right.$ lit. $\left.^{20} \mathrm{mp}=40-41^{\circ} \mathrm{C}\right) . \mathrm{R}_{\mathrm{f}}=0.37(\mathrm{PE}: \mathrm{EtOAC}=5: 1)$.

${ }^{1} \mathrm{H}$ NMR (300 MHz, $\left.\mathrm{CDCl}_{3}, \delta\right): 7.94-7.88(\mathrm{~m}, 2 \mathrm{H}), 7.66-7.56(\mathrm{~m}, 1 \mathrm{H}), 7.48(\mathrm{t}, \mathrm{J}=7.5 \mathrm{~Hz}, 2 \mathrm{H})$, $5.33(\mathrm{~s}, 2 \mathrm{H}), 2.22(\mathrm{~s}, 3 \mathrm{H})$.

${ }^{13} \mathrm{C}$ NMR $\left(75 \mathrm{MHz}, \mathrm{CDCl}_{3}, \delta\right):$ 192.3, 170.5, 134.4, 134.0, 129.0, 127.9, 66.1, 20.7. 
roH

Colorless crystals, $\mathrm{mp}=55^{\circ} \mathrm{C}$ (lit. $\left.{ }^{19} \mathrm{mp}=54-56{ }^{\circ} \mathrm{C}\right) . \mathrm{R}_{\mathrm{f}}=0.33(\mathrm{PE}:$ EtOAC $=5: 1$ ).

${ }^{1} \mathrm{H}$ NMR (300 MHz, $\left.\mathrm{CDCl}_{3}, \delta\right): 7.96-7.87(\mathrm{~m}, 2 \mathrm{H}), 7.63(\mathrm{t}, J=7.5 \mathrm{~Hz}, 1 \mathrm{H}), 7.50$ (t, J= $7.5 \mathrm{~Hz}$, $2 \mathrm{H}), 4.88$ (s, 2H), 3.55 (br.s, $1 \mathrm{H})$.

${ }^{13} \mathrm{C} \mathrm{NMR}\left(75 \mathrm{MHz}, \mathrm{CDCl}_{3}, \delta\right): 198.5,134.4,133.5,129.1,127.8,65.6$.

\section{Experimental procedure for Scheme $4 \mathrm{~b}$.}

An undivided cell was equipped with a platinum plate anode $\left(3 \mathrm{~cm}^{2}\right)$ and a platinum plate cathode $\left(3 \mathrm{~cm}^{2}\right)$ and connected to a DC regulated power supply. The solution of enol acetate $1 \mathrm{a}(1.0 \mathrm{mmol}$, $162.0 \mathrm{mg})$, TEMPO (1.0 mmol, $156.3 \mathrm{mg})$, and $\mathrm{CF}_{3} \mathrm{SO}_{2} \mathrm{Na}(2.0 \mathrm{mmol}, 312.0 \mathrm{mg}) \mathrm{in} 10 \mathrm{~mL}$ of $\mathrm{CH}_{3} \mathrm{CN} / \mathrm{H}_{2} \mathrm{O}$ (8:2) was electrolyzed using constant current conditions $\left(\mathrm{j}=6.7 \mathrm{~mA} / \mathrm{cm}^{2}\right)$ at $20-25$ ${ }^{\circ} \mathrm{C}$ under magnetic stirring for $241 \mathrm{~min}$ with I = $20 \mathrm{~mA}$. Then electrodes were washed with $\mathrm{CH}_{2} \mathrm{Cl}_{2}$ $(2 \times 20 \mathrm{~mL})$. The combined organic phases were washed with brine $(3 \times 10 \mathrm{~mL})$, dried over $\mathrm{MgSO}_{4}$, filtered and concentrated under reduced pressure using a rotary evaporator (15-20 $\mathrm{mmHg}$ ), (bath temperature, ca. $20-25^{\circ} \mathrm{C}$ ). Product $\mathbf{2 a}$ was isolated by chromatography on $\mathrm{SiO}_{2}$ (PE:EtOAc = from 20:1 to 2:1).

\section{Experimental procedure for Scheme 4c.}

A divided cell was equipped with a platinum plate anode $\left(3 \mathrm{~cm}^{2}\right)$ and a platinum plate cathode (3 $\mathrm{cm}^{2}$ ) and connected to a DC regulated power supply. Anodic space: the solution of enol acetate $1 \mathrm{a}(1.0 \mathrm{mmol}, 162.0 \mathrm{mg}), \mathrm{CF}_{3} \mathrm{SO}_{2} \mathrm{Na}(2.0 \mathrm{mmol}, 312.0 \mathrm{mg})$ and $n-\mathrm{Bu}_{4} \mathrm{NBF}_{4}(0.5 \mathrm{mmol}, 164.6 \mathrm{mg})$ in $10 \mathrm{~mL}$ of $\mathrm{CH}_{3} \mathrm{CN} / \mathrm{H}_{2} \mathrm{O}$ (8:2). Cathodic space: $n$-Bu $4 \mathrm{NBF}_{4}(0.5 \mathrm{mmol}, 164.6 \mathrm{mg})$ in $10 \mathrm{~mL}$ of $\mathrm{CH}_{3} \mathrm{CN} / \mathrm{H}_{2} \mathrm{O}$ (8:2). The solutions were electrolyzed using constant current conditions $(\mathrm{j}=6.7$ $\mathrm{mA} / \mathrm{cm}^{2}$ ) at $20-25{ }^{\circ} \mathrm{C}$ under magnetic stirring for $241 \mathrm{~min}$ with I $=20 \mathrm{~mA}$. Then electrodes were washed with $\mathrm{CH}_{2} \mathrm{Cl}_{2}(2 \times 20 \mathrm{~mL})$. The combined organic phases were washed with brine $(3 \times 10$ $\mathrm{mL}$ ), dried over $\mathrm{MgSO}_{4}$, filtered and concentrated under reduced pressure using a rotary evaporator (15-20 mmHg), (bath temperature, ca. $\left.20-25{ }^{\circ} \mathrm{C}\right)$. Products $2 \mathrm{a}(28 \%, 52.6 \mathrm{mg}, 0.28$ $\mathrm{mmol}), 4 a(12 \%, 21.4 \mathrm{mg}, 0.12 \mathrm{mmol}), 5 \mathrm{a}(7 \%, 9.5 \mathrm{mg}, 0.07 \mathrm{mmol})$ and acetophenone (40\%, $48.0 \mathrm{mg}, 0.4 \mathrm{mmol})$ were isolated by chromatography on $\mathrm{SiO}_{2}(\mathrm{PE}: \mathrm{EtOAc}=$ from 20:1 to 2:1).

\section{Experimental procedure for Scheme 4d}

\section{Variant 1}

An undivided cell was equipped with a platinum plate anode $\left(3 \mathrm{~cm}^{2}\right)$ and a platinum plate cathode $\left(3 \mathrm{~cm}^{2}\right)$ and connected to a DC regulated power supply. The solution of enol acetate $1 \mathrm{a}(1.0 \mathrm{mmol}$, $162.0 \mathrm{mg}), \mathrm{CF}_{3} \mathrm{SO}_{2} \mathrm{Na}(2.0 \mathrm{mmol}, 312.0 \mathrm{mg})$, and $n$-Bu4 $\mathrm{NBF}_{4}(0.5 \mathrm{mmol}, 164.6 \mathrm{mg})$ in $10 \mathrm{~mL}$ of $\mathrm{CH}_{3} \mathrm{CN}$ was electrolyzed using constant current conditions $\left(\mathrm{j}=6.7 \mathrm{~mA} / \mathrm{cm}^{2}\right)$ at $20-25{ }^{\circ} \mathrm{C}$ under magnetic stirring for $241 \mathrm{~min}$ with I = $20 \mathrm{~mA}$ under an inert atmosphere (Ar). Then electrodes 
were washed with $\mathrm{CH}_{2} \mathrm{Cl}_{2}(2 \times 20 \mathrm{~mL})$. The combined organic phases were washed with brine (3 $\times 10 \mathrm{~mL}$ ), dried over $\mathrm{MgSO}_{4}$, filtered and concentrated under reduced pressure using a rotary evaporator $(15-20 \mathrm{mmHg})$, (bath temperature, ca. $\left.20-25{ }^{\circ} \mathrm{C}\right)$. Product $2 \mathrm{a}$ was isolated by chromatography on $\mathrm{SiO}_{2}(\mathrm{PE}: \mathrm{EtOAc}=$ from 20:1 to 2:1).

\section{Variant 2}

An undivided cell was equipped with a platinum plate anode $\left(3 \mathrm{~cm}^{2}\right)$ and a platinum plate cathode $\left(3 \mathrm{~cm}^{2}\right)$ and connected to a DC regulated power supply. The solution of enol acetate $1 \mathrm{a}(1.0 \mathrm{mmol}$, $162.0 \mathrm{mg})$, and $\mathrm{CF}_{3} \mathrm{SO}_{2} \mathrm{Na}(2.0 \mathrm{mmol}, 312.0 \mathrm{mg})$ in $10 \mathrm{~mL}$ of $\mathrm{CH}_{3} \mathrm{CN} / \mathrm{H}_{2} \mathrm{O}$ (8:2) was electrolyzed using constant current conditions $\left(\mathrm{j}=6.7 \mathrm{~mA} / \mathrm{cm}^{2}\right)$ at $20-25^{\circ} \mathrm{C}$ under magnetic stirring for $241 \mathrm{~min}$ with I = $20 \mathrm{~mA}$ under an inert atmosphere (Ar). Then electrodes were washed with $\mathrm{CH}_{2} \mathrm{Cl}_{2}(2 \times$ $20 \mathrm{~mL})$. The combined organic phases were washed with brine $(3 \times 10 \mathrm{~mL})$, dried over $\mathrm{MgSO}_{4}$, filtered and concentrated under reduced pressure using a rotary evaporator (15-20 mmHg), (bath temperature, ca. $\left.20-25^{\circ} \mathrm{C}\right)$. Product $2 \mathrm{a}$ was isolated by chromatography on $\mathrm{SiO}_{2}(\mathrm{PE}: \mathrm{EtOAc}=$ from $20: 1$ to $2: 1)$.

\section{CV data of TEMPO and $\mathrm{CF}_{3} \mathrm{SO}_{2} \mathrm{Na}$}

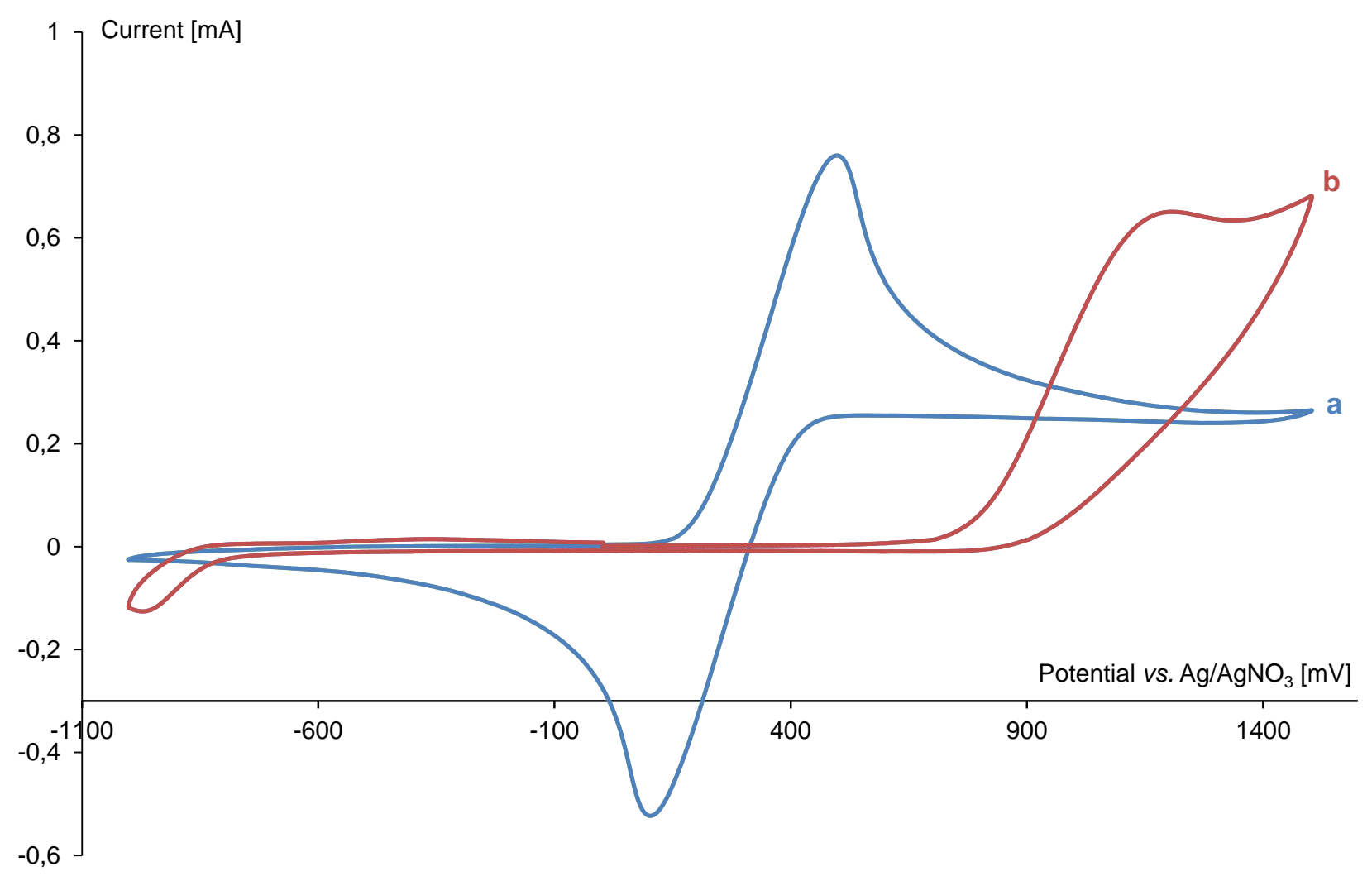

Figure S1. CV curves on a working glassy-carbon electrode $(\mathrm{d}=3 \mathrm{~mm})$ under a scan rate of $0.1 \mathrm{~V} / \mathrm{s}$ for (a) TEMPO $(0.05 \mathrm{M})$; (b) $\mathrm{CF}_{3} \mathrm{SO}_{2} \mathrm{Na}(0.05 \mathrm{M})$ in $0.1 \mathrm{M} n-\mathrm{Bu}_{4} \mathrm{NBF}_{4}$ solution in $\mathrm{CH}_{3} \mathrm{CN}: \mathrm{H}_{2} \mathrm{O}(8: 2)$. 
$\mathrm{CV}$ data of deaerated and aerated mixtures of $\mathrm{CF}_{3} \mathrm{SO}_{2} \mathrm{Na}$ and enol acetate

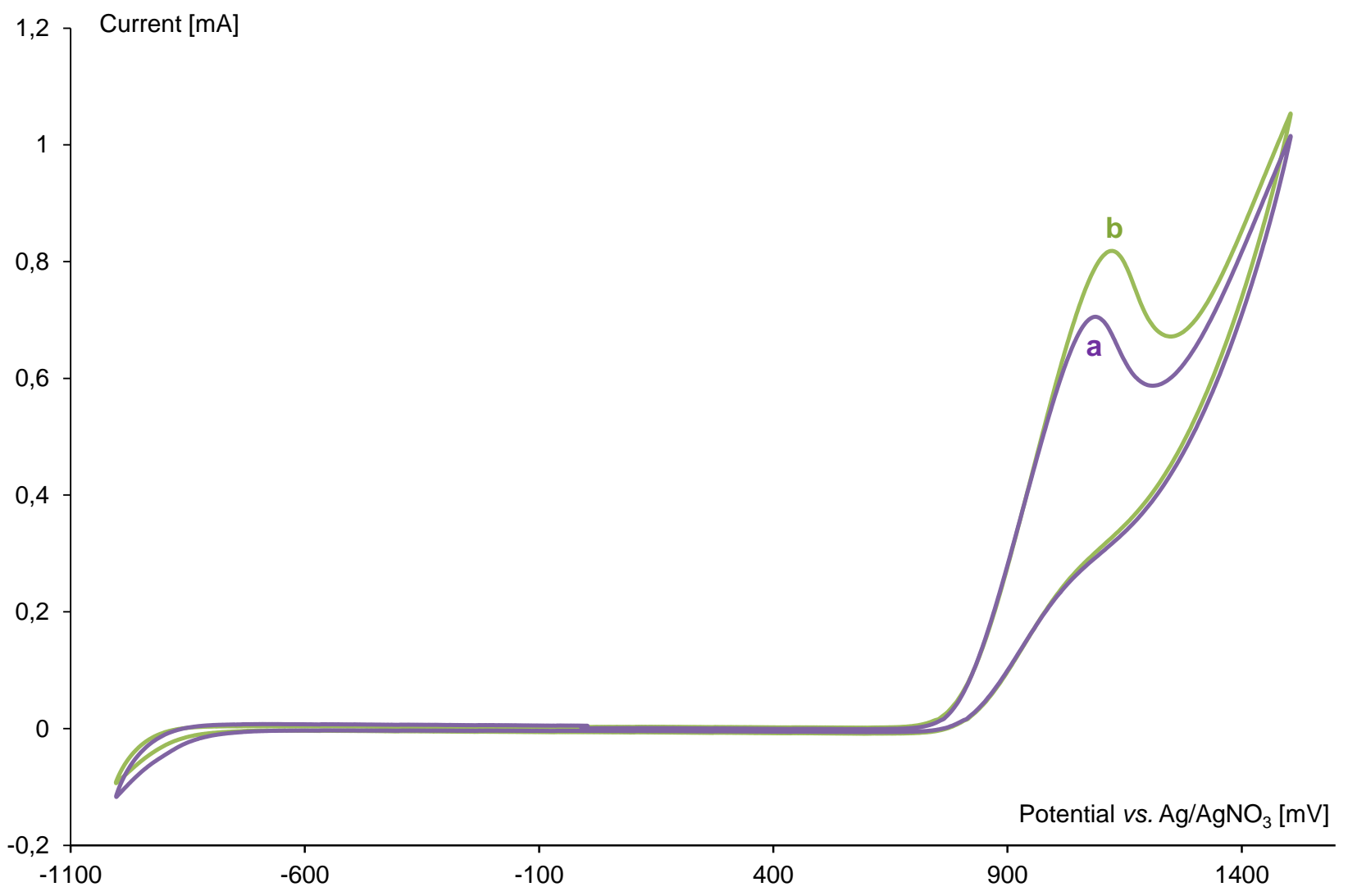

Figure S2. CV curves on a working glassy-carbon electrode $(\mathrm{d}=3 \mathrm{~mm})$ under a scan rate of $0.1 \mathrm{~V} / \mathrm{s}$ for (a) aerated $\mathrm{CF}_{3} \mathrm{SO}_{2} \mathrm{Na}(0.05 \mathrm{M})$ and enol acetate $1 \mathrm{I}(0.025 \mathrm{M})$; (b) deaerated $\mathrm{CF}_{3} \mathrm{SO}_{2} \mathrm{Na}(0.05 \mathrm{M})$ and enol acetate $1 \mathrm{I}(0.025 \mathrm{M})$ in $0.1 \mathrm{M} n-\mathrm{Bu}_{4} \mathrm{NBF}_{4}$ solution in $\mathrm{CH}_{3} \mathrm{CN}: \mathrm{H}_{2} \mathrm{O}$ (8:2).

The interception of the intermediate radical by the oxygen decreases the current in the case of an aerated reaction mixture. 


\section{References}

1. Tsedilin, A. M.; Fakhrutdinov, A. N.; Eremin, D. B.; Zalesskiy, S. S.; Chizhov, A. O.; Kolotyrkina, N. G.; Ananikov, V. P., How sensitive and accurate are routine NMR and MS measurements? Mendeleev Commun. 2015, 25 (6), 454-456.

2. Miura, K.; Fujisawa, N.; Saito, H.; Wang, D.; Hosomi, A., Synthetic utility of stannyl enolates as radical alkylating agents. Org. Lett. 2001, 3 (16), 2591-2594.

3. Lu, Y.; Li, Y.; Zhang, R.; Jin, K.; Duan, C., Highly efficient Cu(I)-catalyzed trifluoromethylation of aryl(heteroaryl) enol acetates with CF3 radicals derived from CF3SO2Na and TBHP at room temperature. J. Fluorine Chem. 2014, 161, 128-133.

4. He, R. D.; Li, C. L.; Pan, Q. Q.; Guo, P.; Liu, X. Y.; Shu, X. Z., Reductive Coupling between C-N and C-O Electrophiles. J. Am. Chem. Soc. 2019, 141 (32), 12481-12486.

5. Song, C. X.; Cai, G. X.; Farrell, T. R.; Jiang, Z. P.; Li, H.; Gan, L. B.; Shi, Z. J., Direct functionalization of benzylic C-Hs with vinyl acetates via Fe-catalysis. Chem. Commun. 2009, (40), 6002-6004.

6. Gartner, D.; Stein, A. L.; Grupe, S.; Arp, J.; Jacobi von Wangelin, A., Iron-Catalyzed CrossCoupling of Alkenyl Acetates. Angewandte Chemie-International Edition 2015, 54 (36), 1054510549.

7. Geibel, I.; Dierks, A.; Muller, T.; Christoffers, J., Formation of delta-Lactones with antiBaeyer-Villiger Regiochemistry: Investigations into the Mechanism of the Cerium-Catalyzed Aerobic Coupling of beta-Oxoesters with Enol Acetates. Chemistry-a European Journal 2017, 23 (30), 7245-7254.

8. Felipe-Blanco, D.; Gonzalez-Gomez, J. C., Salicylic Acid-Catalyzed Arylation of Enol Acetates with Anilines. Adv. Synth. Catal. 2018, 360 (14), 2773-2778.

9. Bulman Page, P. C.; Almutairi, S. M.; Chan, Y.; Stephenson, G. R.; Gama, Y.; Goodyear, R. L.; Douteau, A.; Allin, S. M.; Jones, G. A., Asymmetric Oxidation of Enol Derivatives to a-Alkoxy Carbonyls Using Iminium Salt Catalysts: A Synthetic and Computational Study. J. Org. Chem. 2019, 84 (2), 544-559.

10. Eames, J.; Coumbarides, G. S.; Suggate, M. J.; Weerasooriya, N., Investigations into the regioselective C-deuteration of acyclic and exocyclic enolates. Eur. J. Org. Chem. 2003, 2003 (4), 634-641.

11. Sharley, J. S.; Perez, A. M. C.; Ferri, E. E.; Miranda, A. F.; Baxendale, I. R., alpha,betaUnsaturated ketones via copper(II) bromide mediated oxidation. Tetrahedron 2016, 72 (22), 29472954.

12. de Souza, A. A. N.; Silva, N. S.; Muller, A. V.; Polo, A. S.; Brocksom, T. J.; de Oliveira, K. T., Porphyrins as Photoredox Catalysts in $\mathrm{Csp}(2)-\mathrm{H}$ Arylations: Batch and Continuous Flow Approaches. J. Org. Chem. 2018, 83 (24), 15077-15086.

13. Das, S.; Hashmi, A. S. K.; Schaub, T., Direct Photoassisted alpha-Trifluoromethylation of Aromatic Ketones with Trifluoroacetic Anhydride (TFAA). Adv. Synth. Catal. 2019, 361 (4), 720724.

14. Pramanik, S.; Rej, S.; Kando, S.; Tsurugi, H.; Mashima, K., Organosilicon Reducing Reagents for Stereoselective Formations of Silyl Enol Ethers from alpha-Halo Carbonyl Compounds. J. Org. Chem. 2018, 83 (4), 2409-2417.

15. Qin, H. T.; Wu, S. W.; Liu, J. L.; Liu, F., Photoredox-catalysed redox-neutral trifluoromethylation of vinyl azides for the synthesis of alpha-trifluoromethylated ketones. Chem. Commun. 2017, 53 (10), 1696-1699. 
16. Kamigata, N.; Udodaira, K.; Shimizu, T., Reactions of perfluoroalkanesulfonyl chlorides with silyl enol ethers catalyzed by a ruthenium(II) phosphine complex. Phosphorus Sulfur and Silicon and the Related Elements 1997, 129, 155-168.

17. Su, X. L.; Huang, H. G.; Yuan, Y. F.; Li, Y., Radical Desulfur-Fragmentation and Reconstruction of Enol Triflates: Facile Access to alpha-Trifluoromethyl Ketones. Angewandte Chemie-International Edition 2017, 56 (5), 1338-1341.

18. Chu, X.-Q.; Xie, T.; Wang, Y.-W.; Li, X.-R.; Rao, W.; Xu, H.; Shen, Z.-L., Synthesis of di(hetero)aryl sulfides by defluorinative sulfenylation of polyfluoroalkyl ketones with sodium sulfinates or arylsulfonyl chlorides. Chem. Commun. 2020, 56 (61), 8699-8702.

19. Zhou, X.; Ma, H.; Cao, J.; Liu, X.; Huang, G., Novel and efficient transformation of enamides into a-acyloxy ketones via an acyl intramolecular migration process. Org. Biomol. Chem. 2016, 14 (42), 10070-10073.

20. Sheng, J.; Li, X.; Tang, M.; Gao, B.; Huang, G., An Efficient Method for the a-Acetoxylation of Ketones. Synthesis 2007, 2007 (08), 1165-1168. 
NMR spectra of starting enol acetates

1-Phenylvinyl acetate, $1 \mathrm{a}$

${ }^{1} \mathrm{H}$ NMR (300 MHz, $\mathrm{CDCl}_{3}$ )

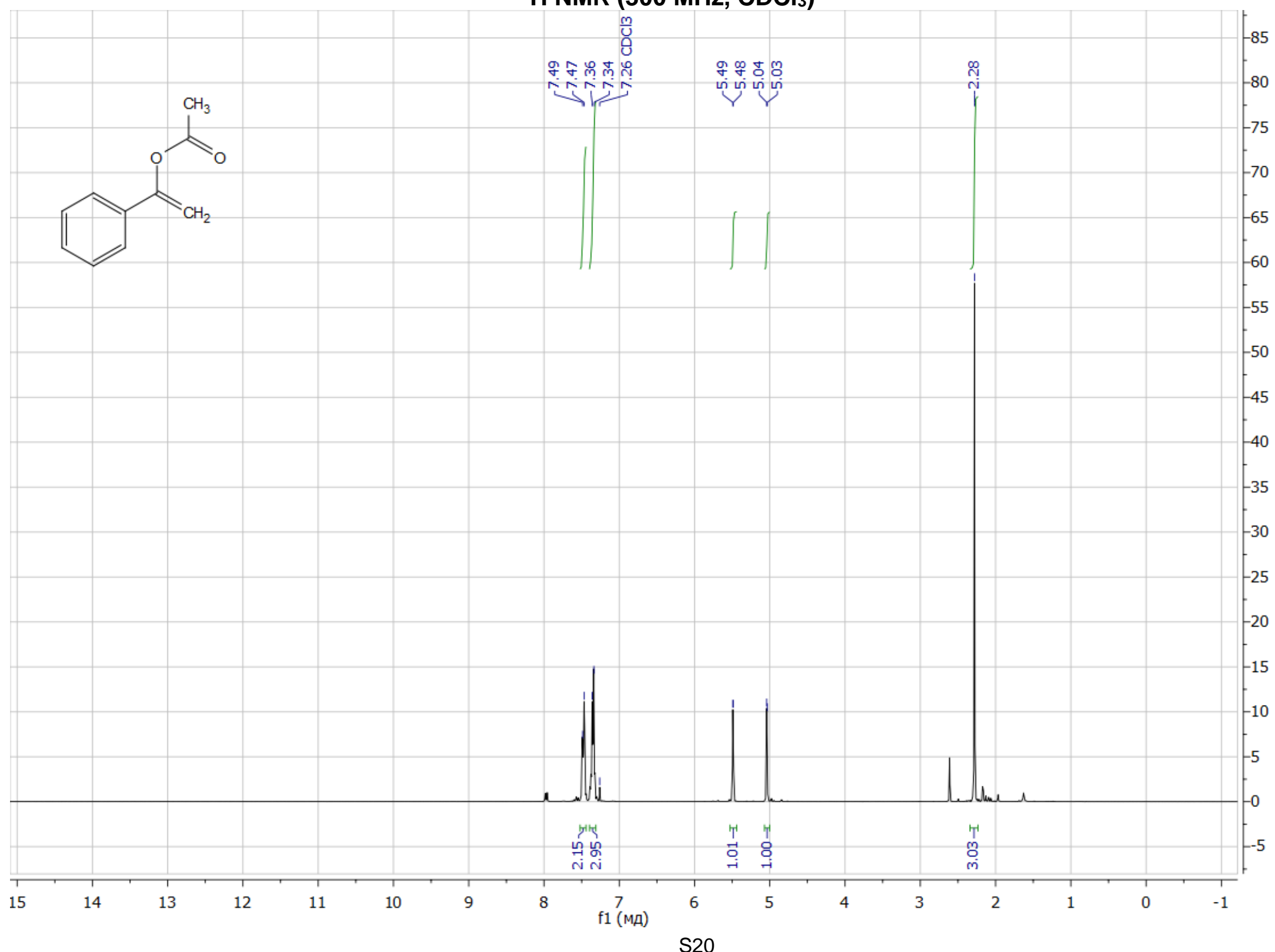


$\left.{ }^{13} \mathrm{C} \mathrm{NMR} \mathrm{(75} \mathrm{MHz,} \mathrm{CDCl}_{3}\right)$

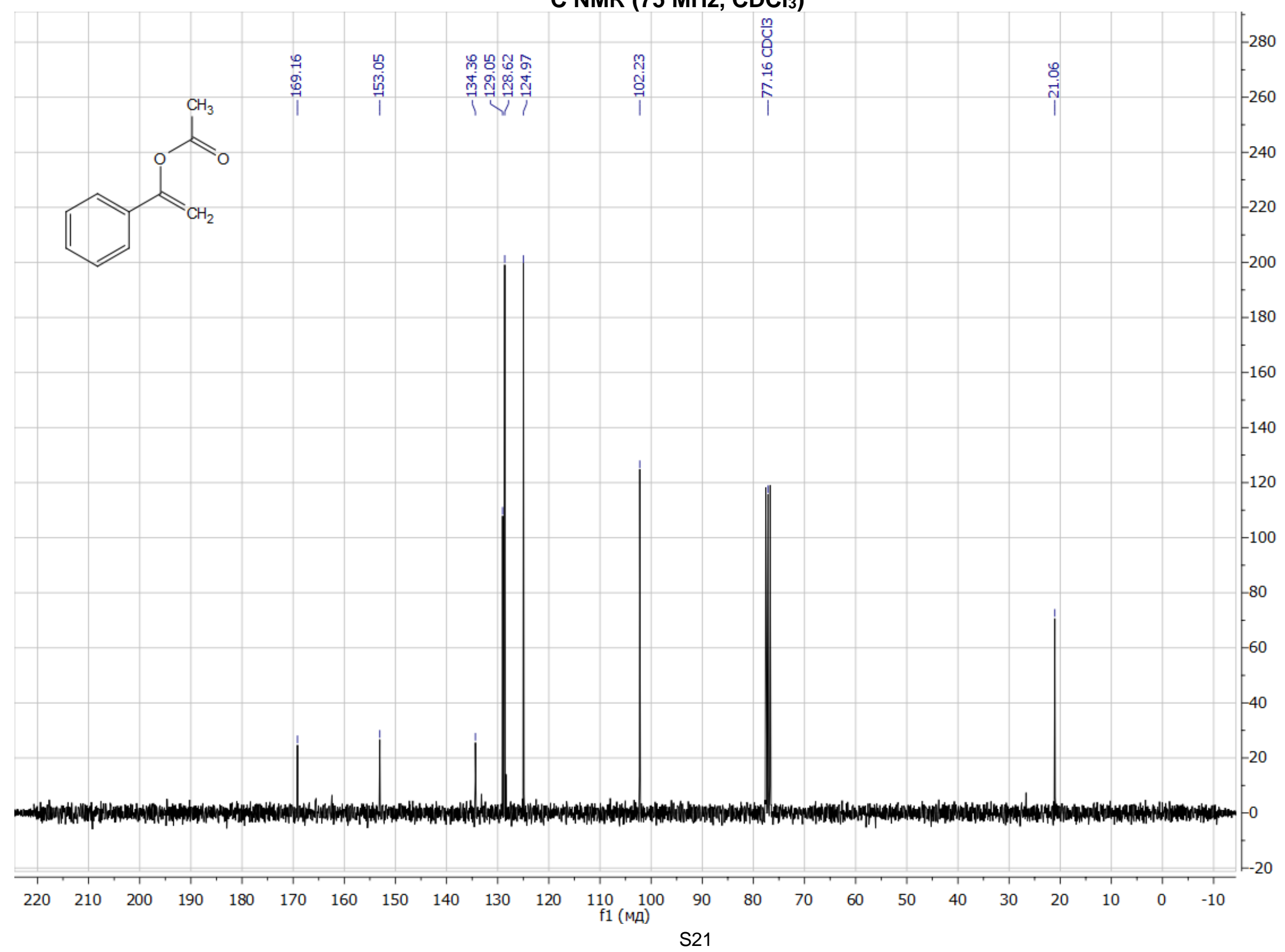


1-(p-Tolyl)vinyl acetate, $1 \mathrm{~b}$

${ }^{1} \mathrm{H}$ NMR (300 MHz, CDCl 3 )

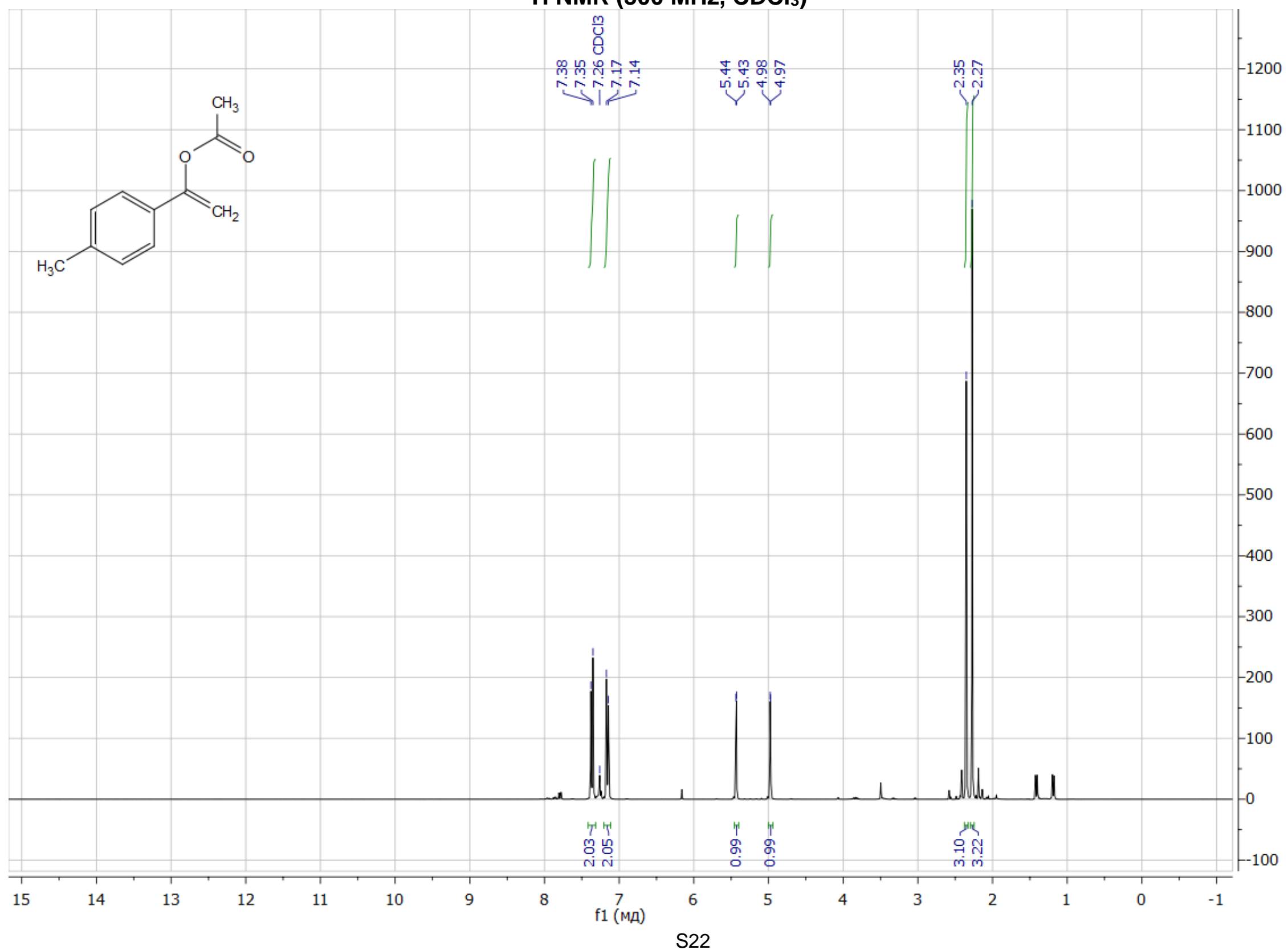


${ }^{13} \mathrm{C}$ NMR (75 MHz, $\left.\mathrm{CDCl}_{3}\right)$

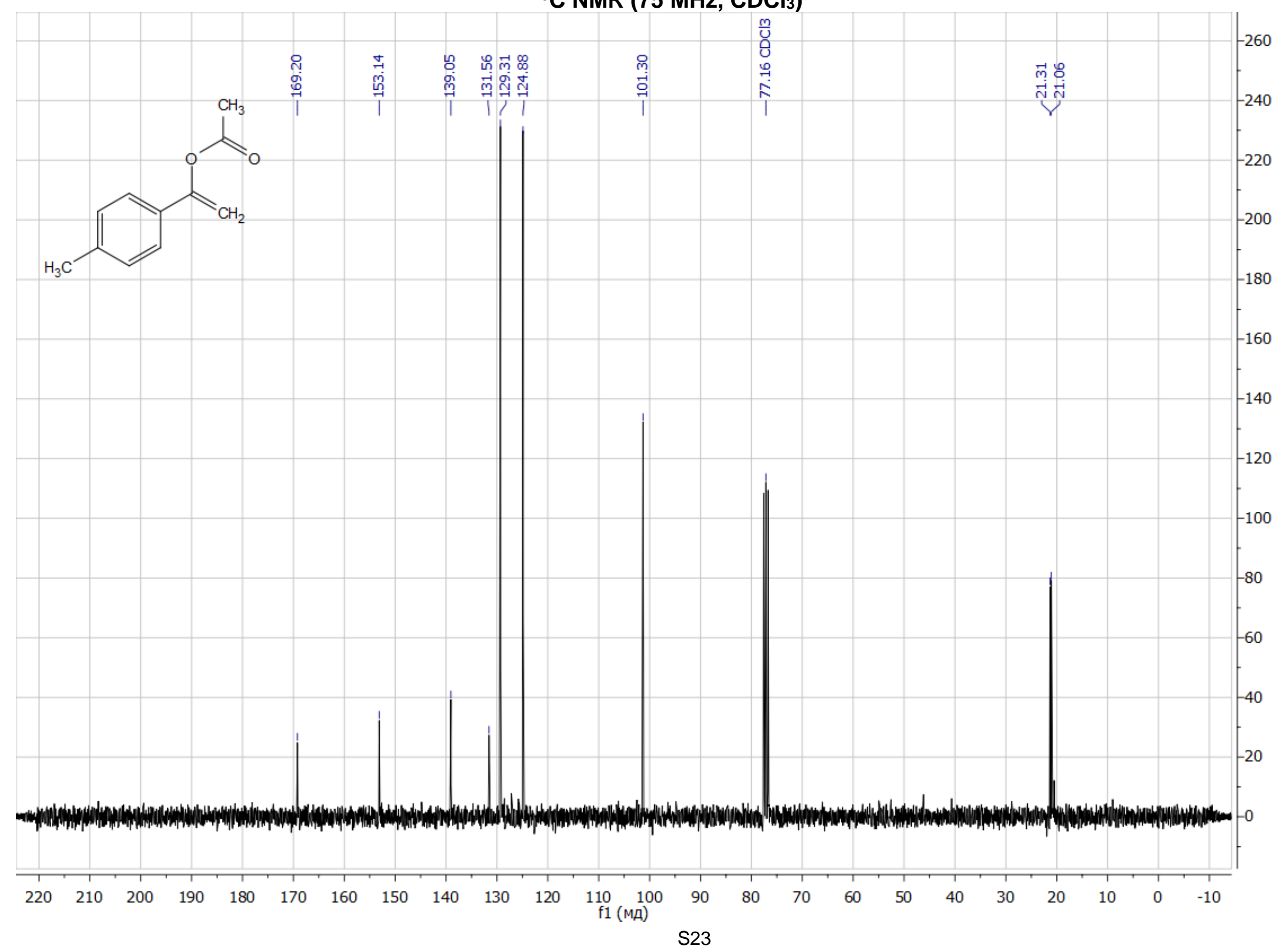


1-(4-Methoxyphenyl)vinyl acetate, 1c

${ }^{1} \mathrm{H}$ NMR (300 MHz, CDCl 3 )

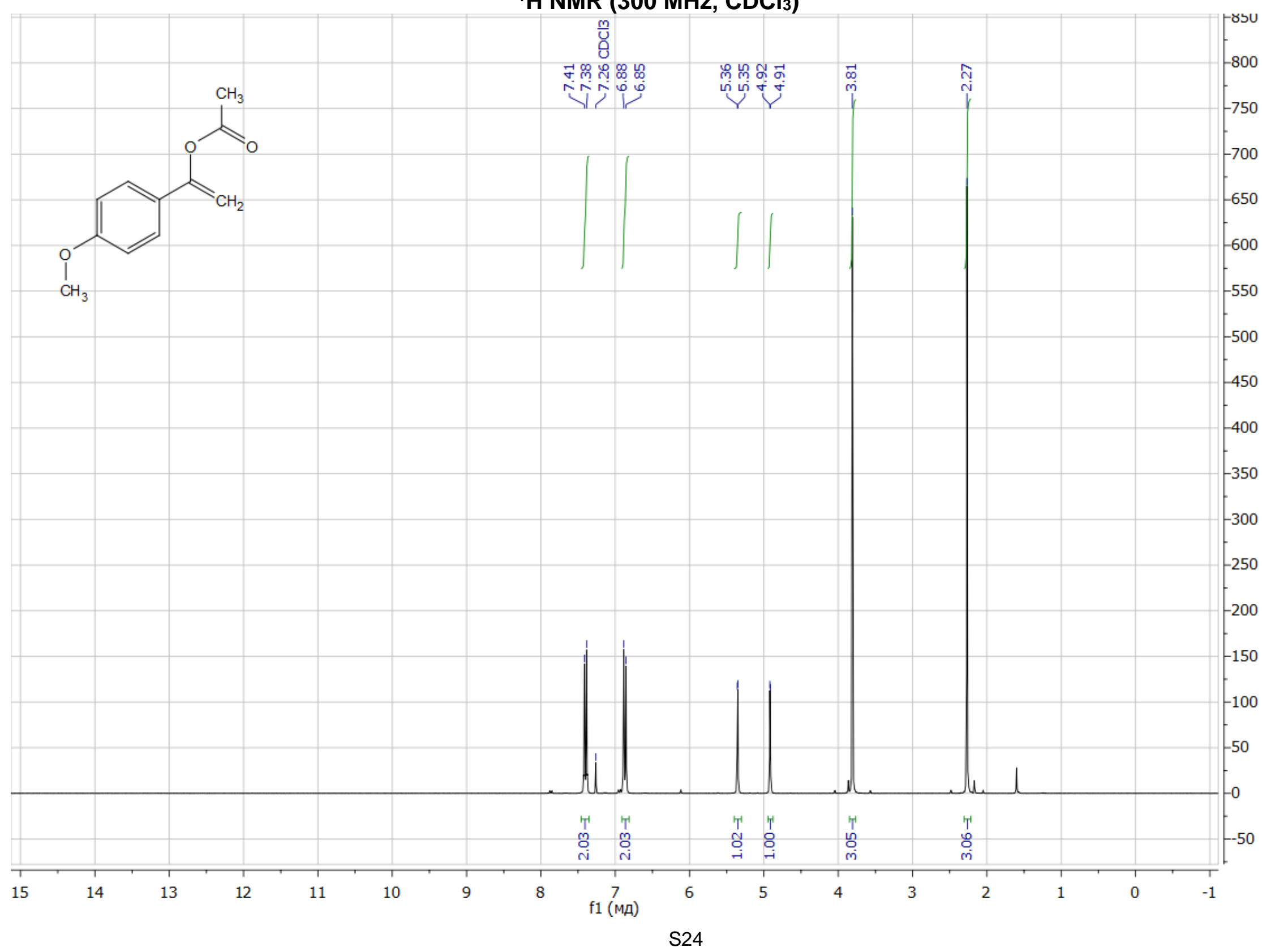


$\left.{ }^{13} \mathrm{C} \mathrm{NMR} \mathrm{(75} \mathrm{MHz,} \mathrm{CDCl}_{3}\right)$

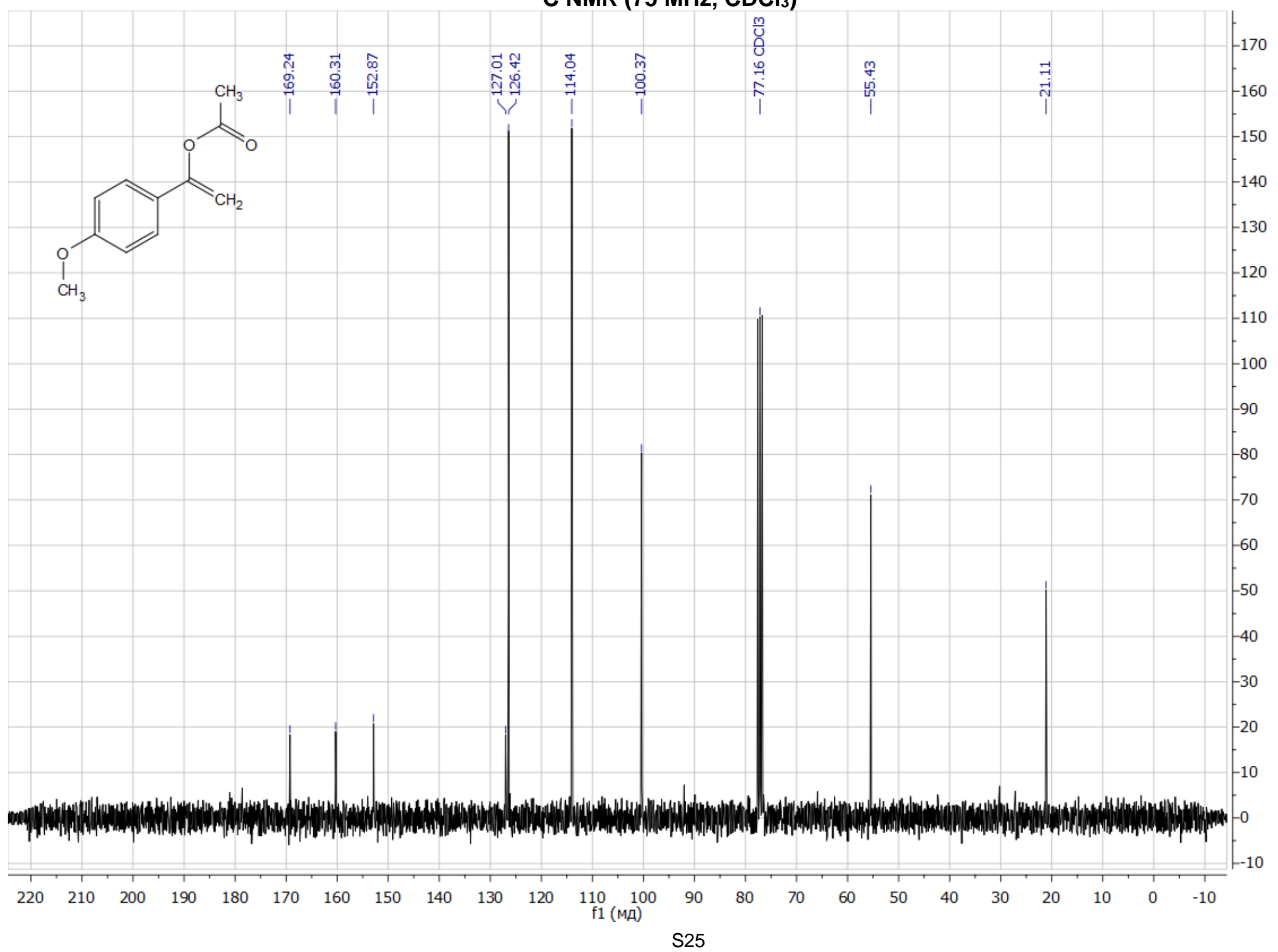


1-(4-Chlorophenyl)vinyl acetate, 1d

${ }^{1} \mathrm{H}$ NMR (300 MHz, $\left.\mathrm{CDCl}_{3}\right)$

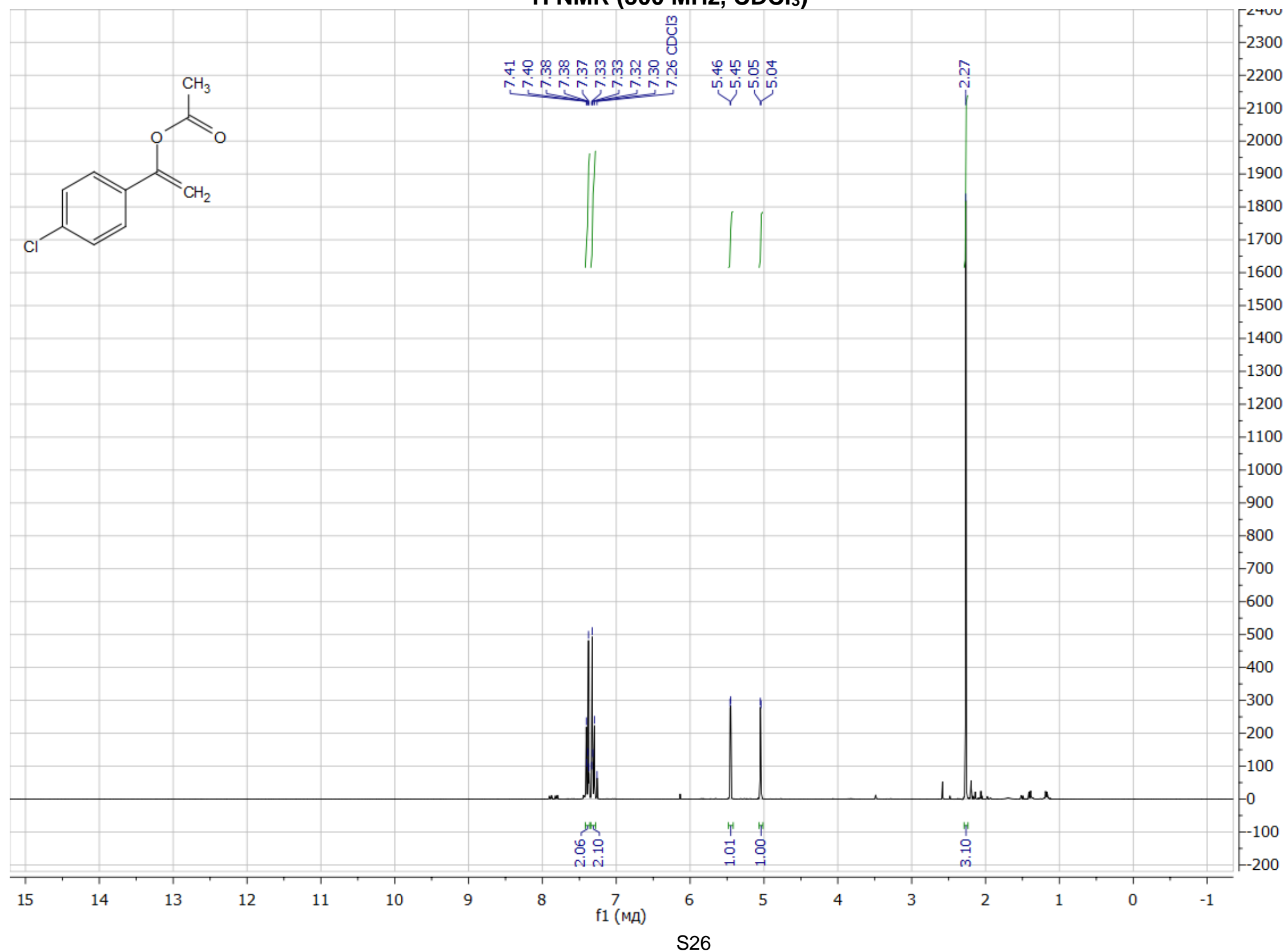


$\left.{ }^{13} \mathrm{C} \mathrm{NMR} \mathrm{(75} \mathrm{MHz,} \mathrm{CDCl}_{3}\right)$

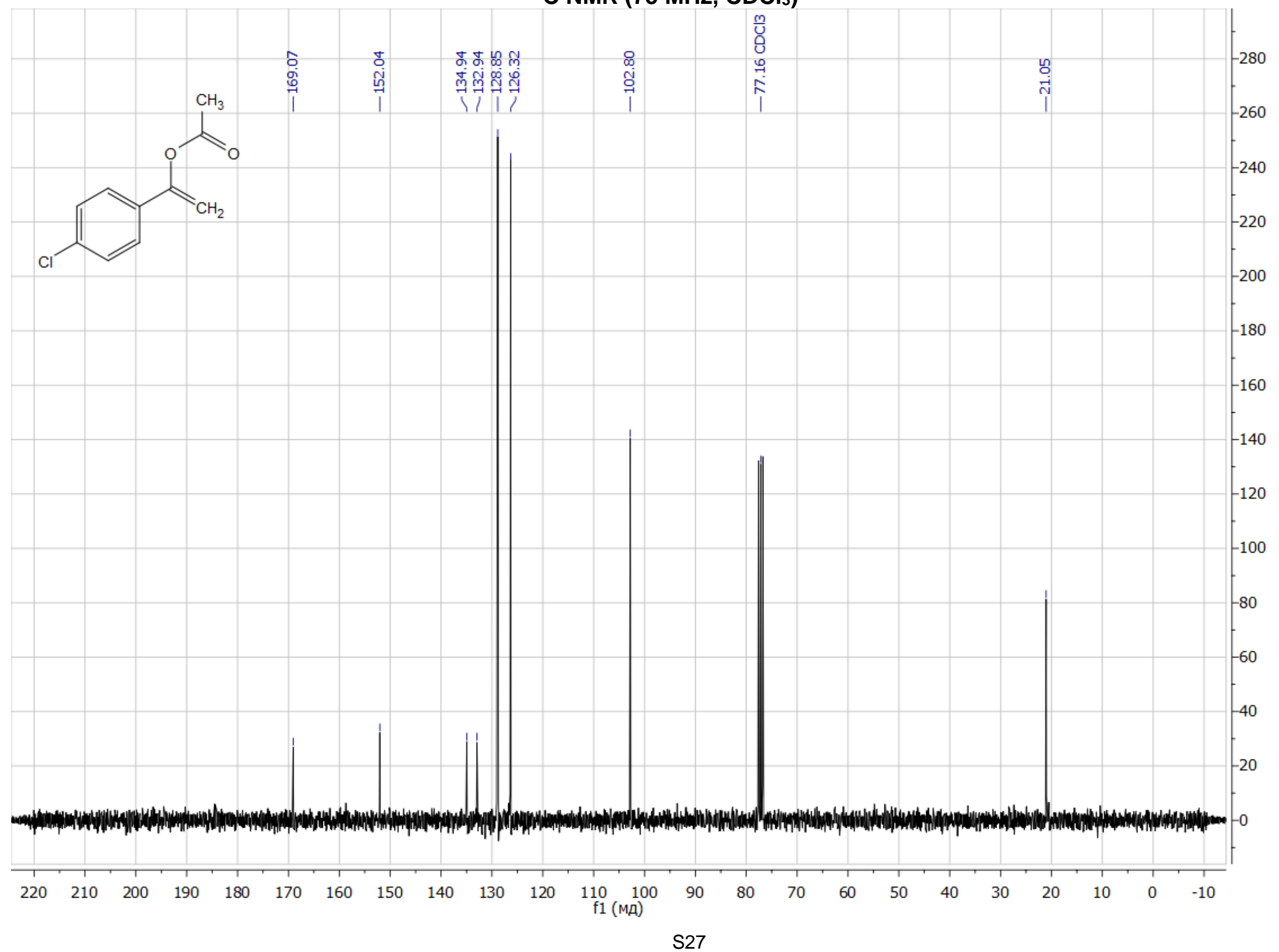


1-(4-Fluorophenyl)vinyl acetate, $1 \mathrm{e}$

${ }^{1} \mathrm{H}$ NMR (300 MHz, $\left.\mathrm{CDCl}_{3}\right)$

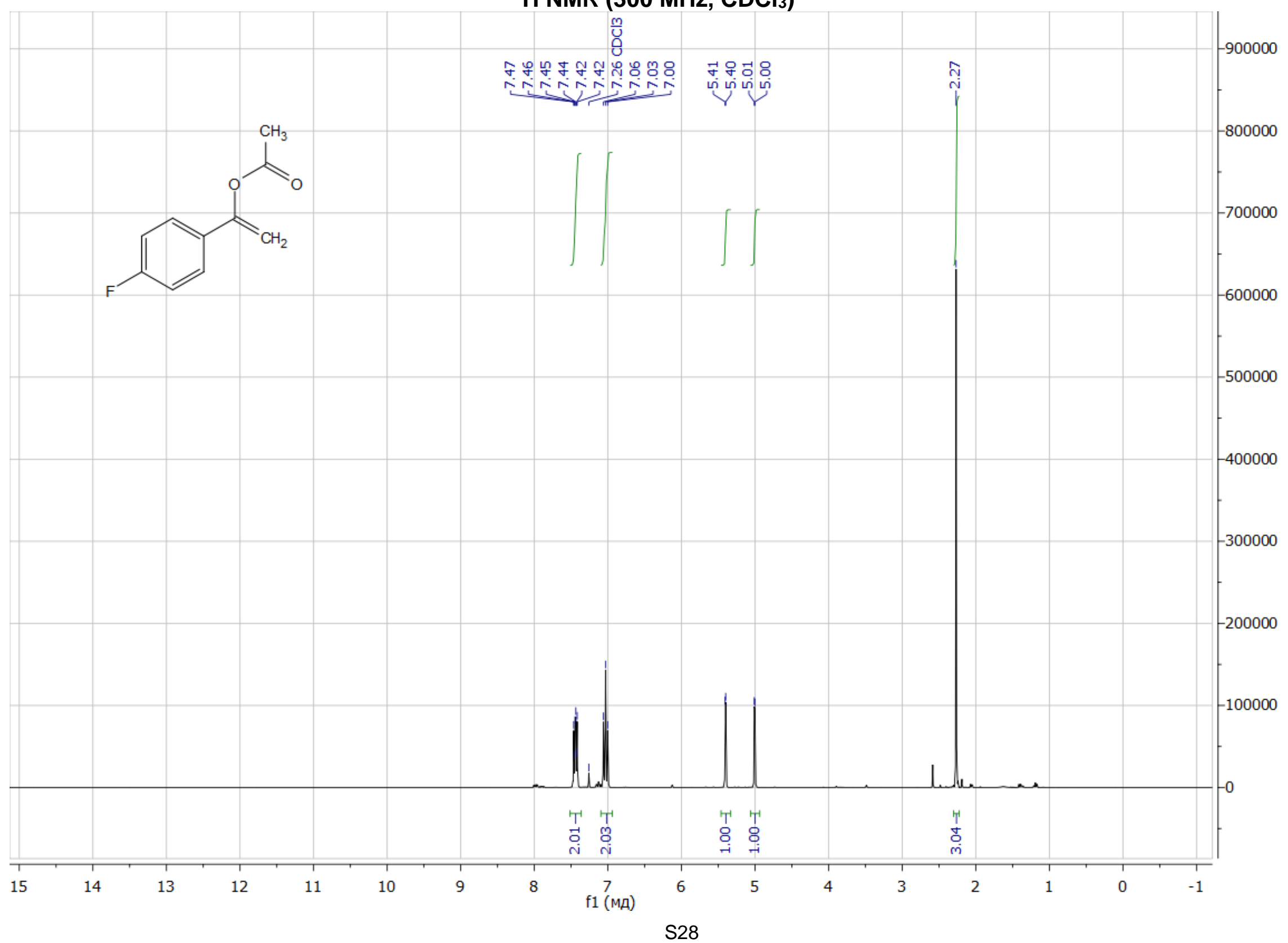


$\left.{ }^{13} \mathrm{C} \mathrm{NMR} \mathrm{(75} \mathrm{MHz,} \mathrm{CDCl}_{3}\right)$

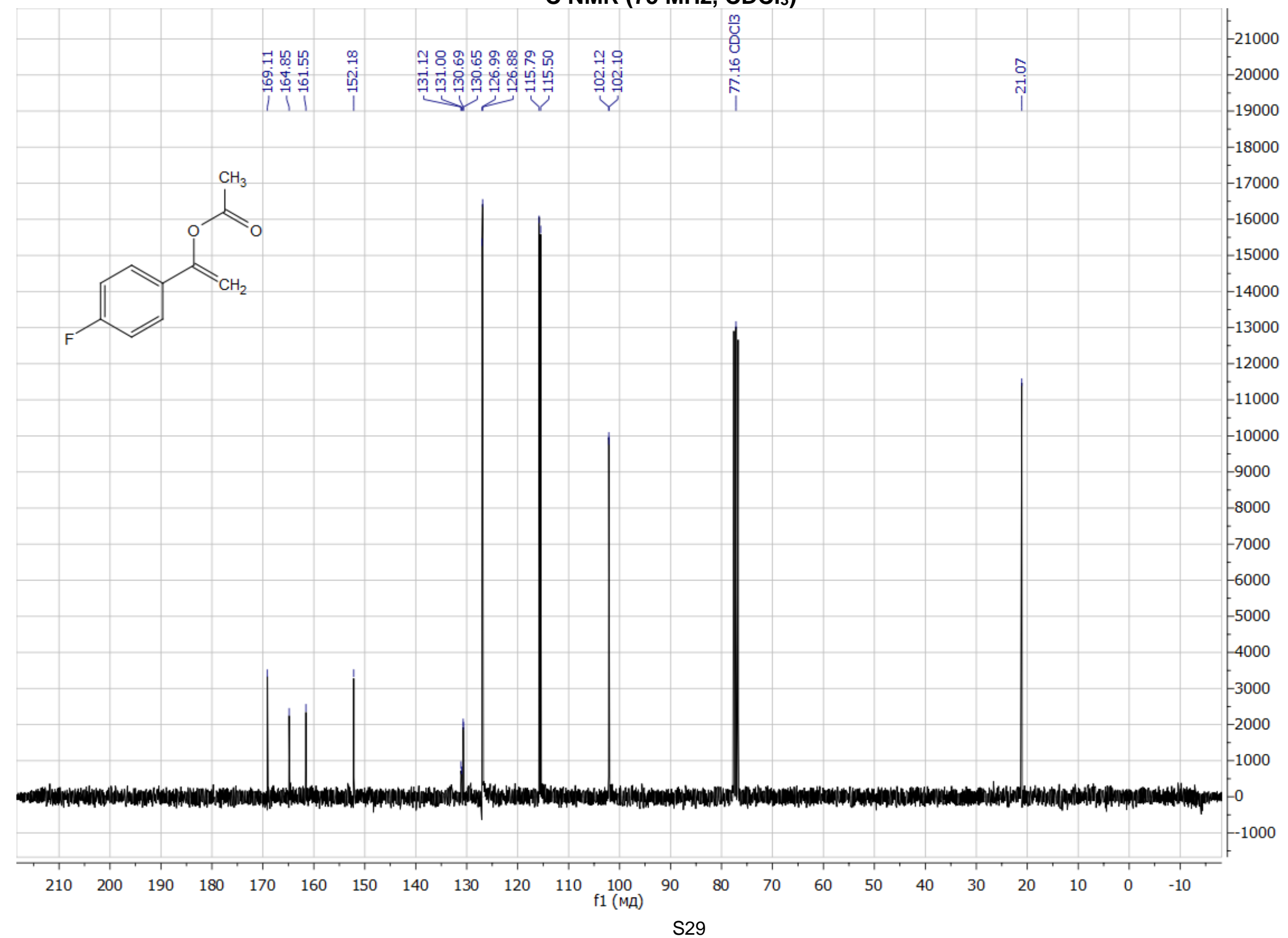


1-(4-Bromophenyl)vinyl acetate, $1 f$

${ }^{1} \mathrm{H}$ NMR (300 MHz, CDCl 3 )

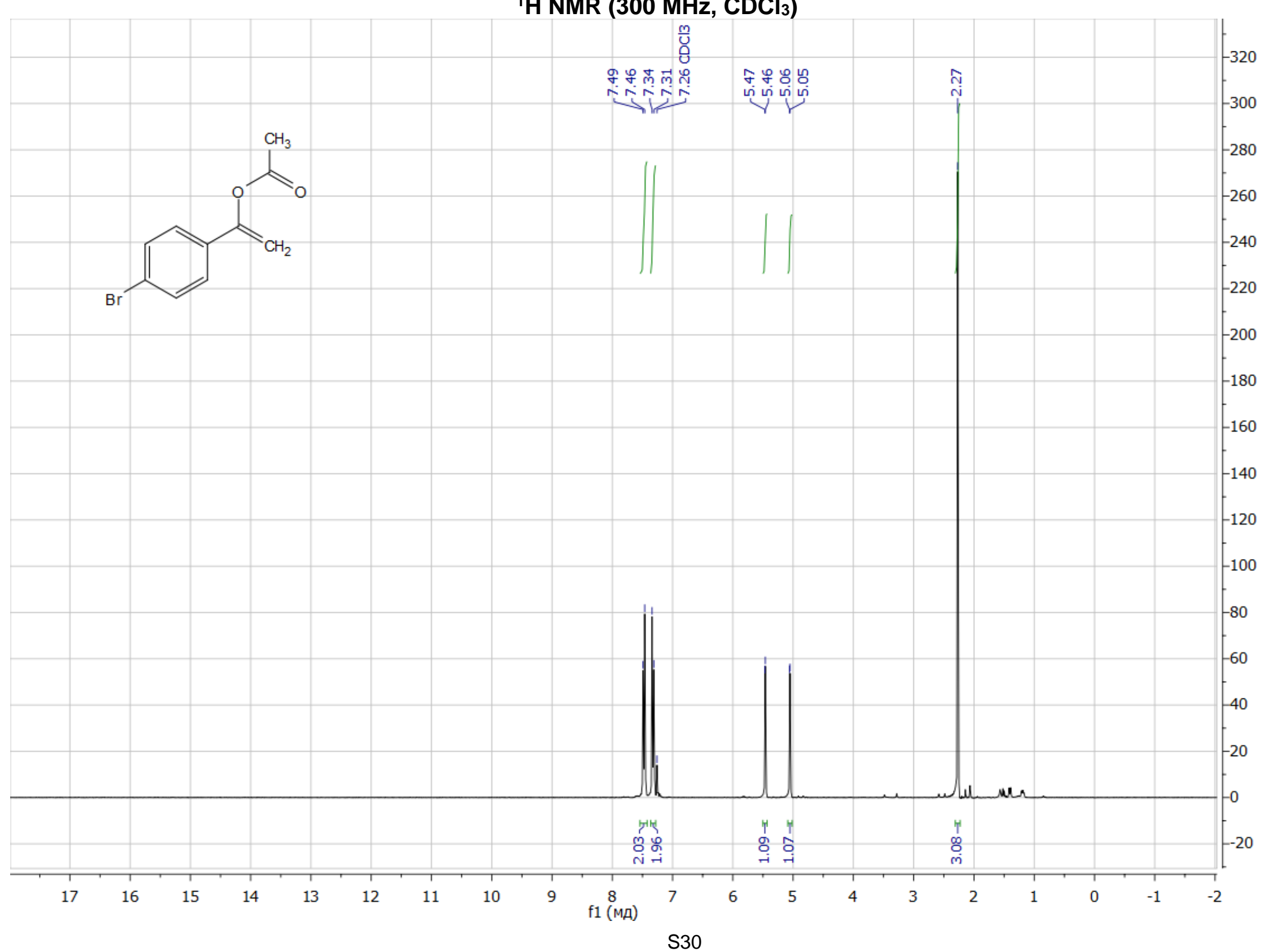


$\left.{ }^{13} \mathrm{C} \mathrm{NMR} \mathrm{(75} \mathrm{MHz,} \mathrm{CDCl}_{3}\right)$

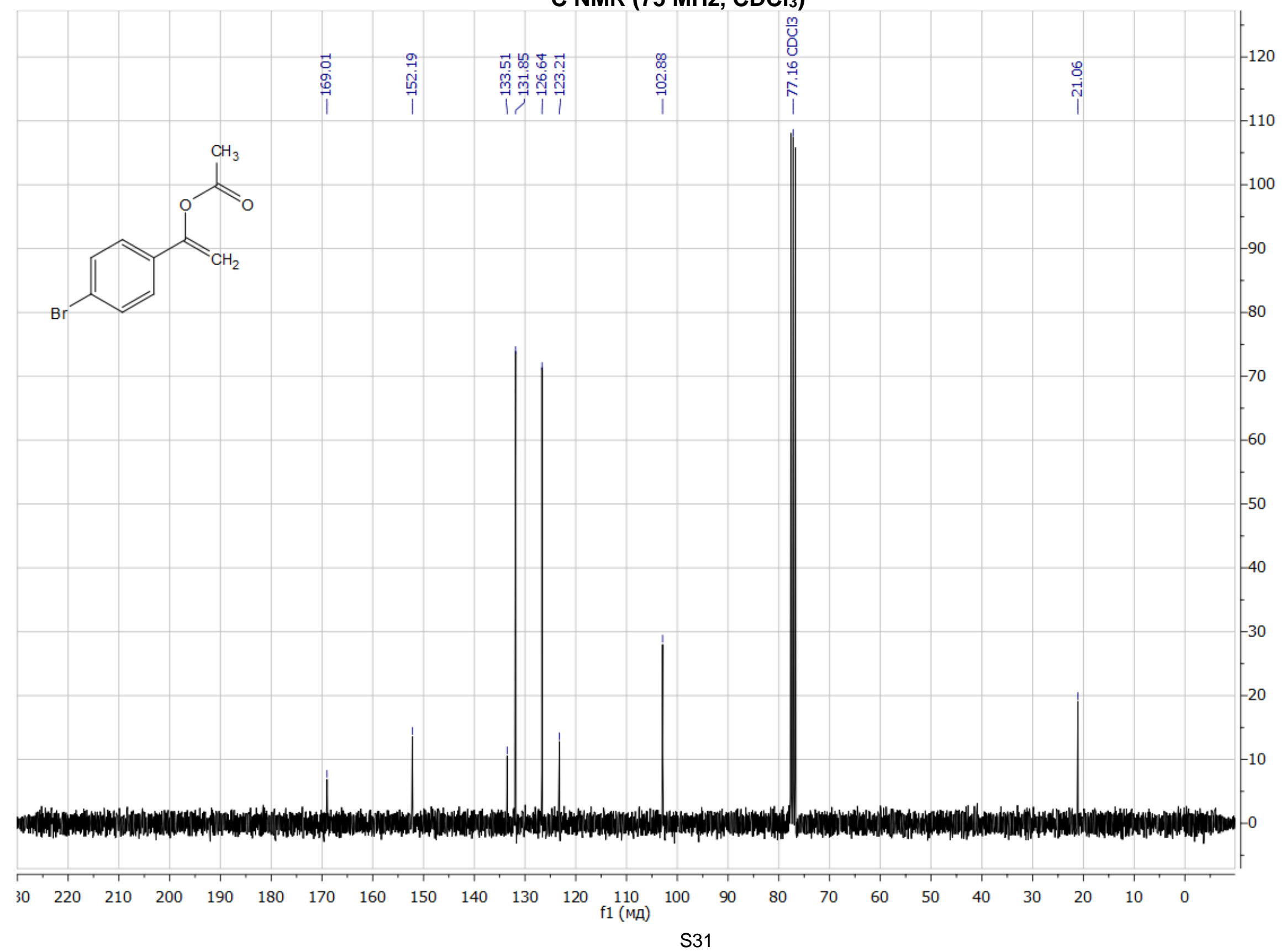


1H-Inden-3-yl acetate, $1 \mathrm{~g}$

${ }^{1} \mathrm{H}$ NMR (300 MHz, CDCl 3 )

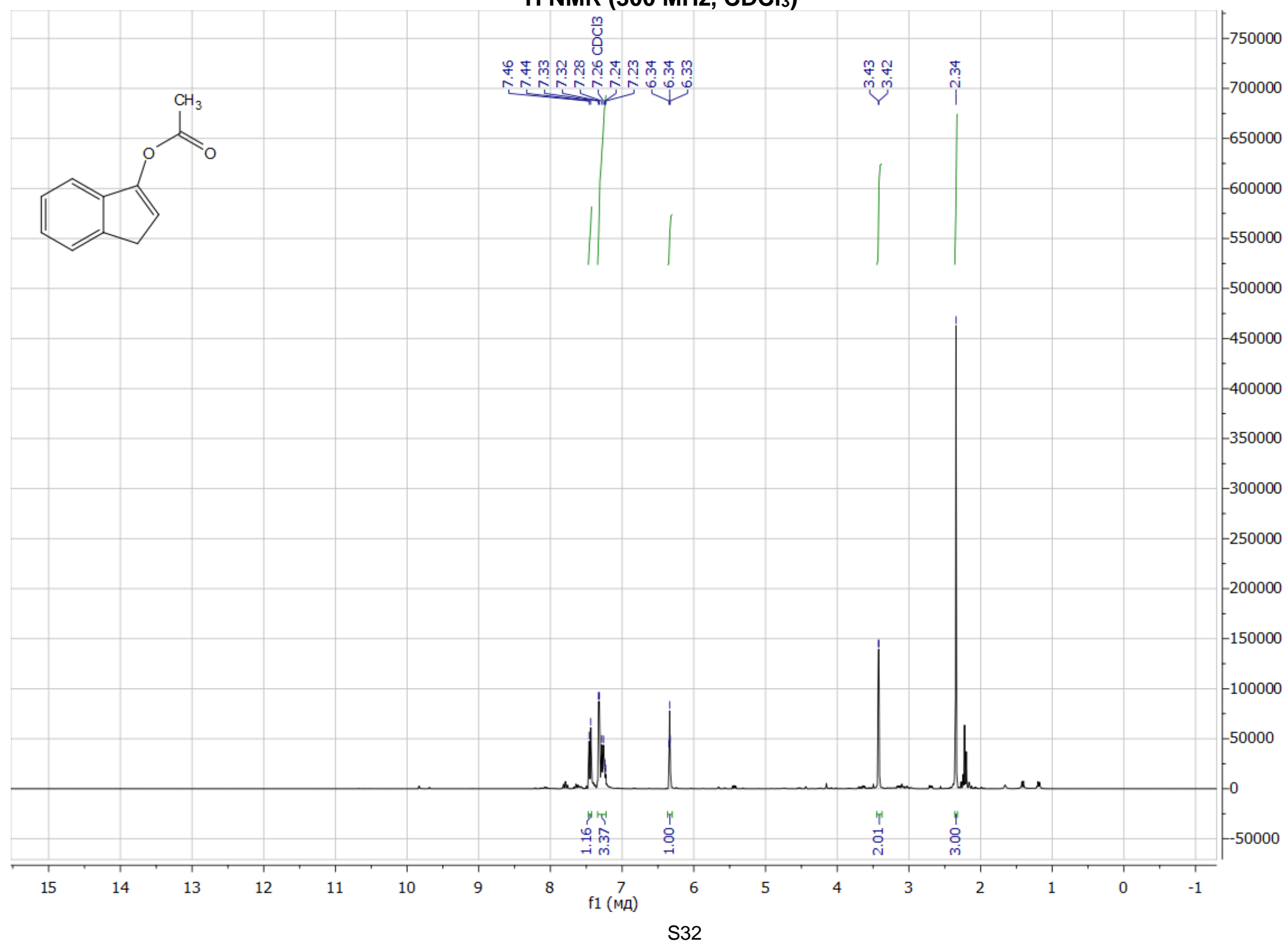


$\left.{ }^{13} \mathrm{C} \mathrm{NMR} \mathrm{(75} \mathrm{MHz,} \mathrm{CDCl}_{3}\right)$

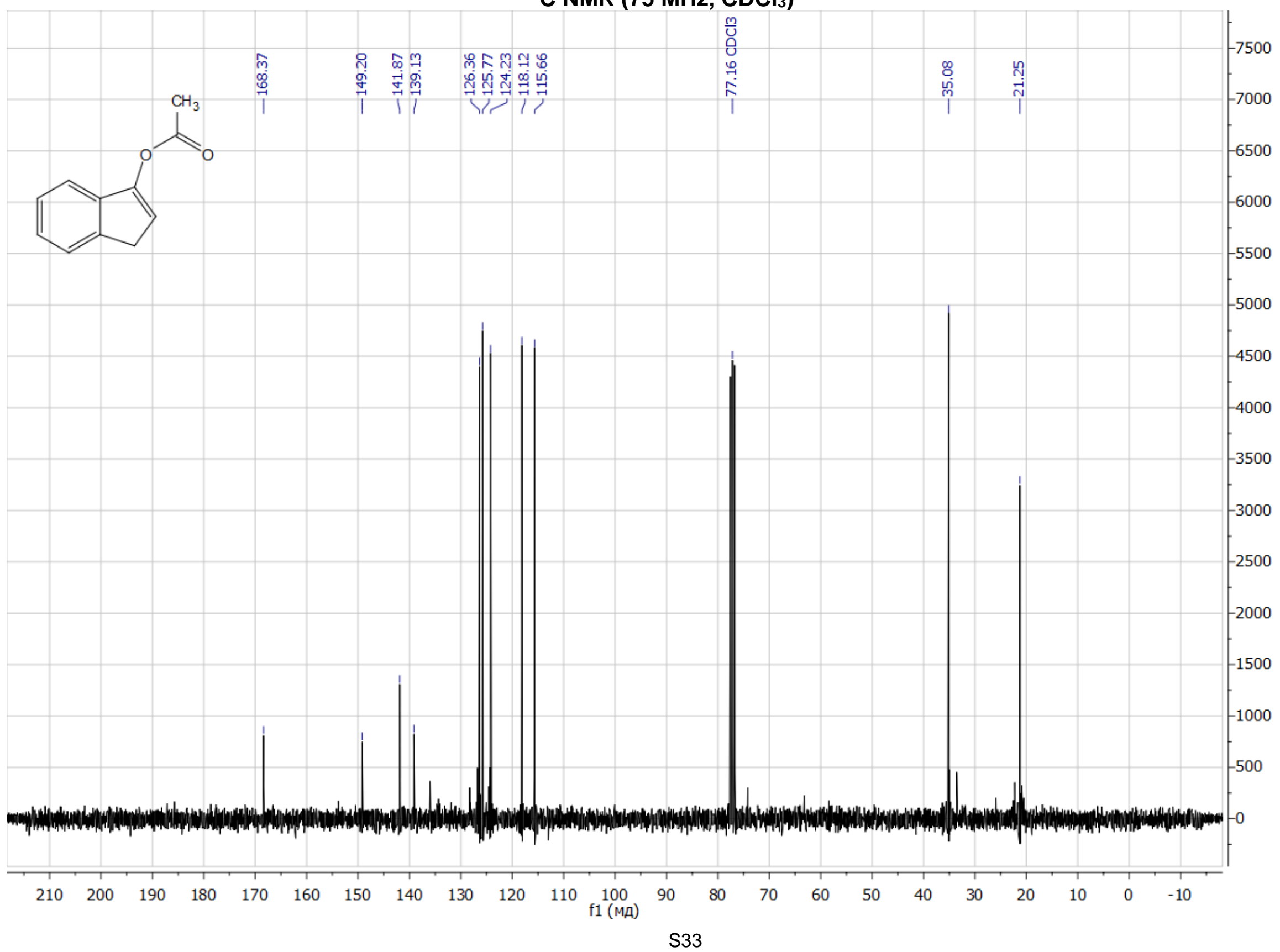


3,4-Dihydronaphthalen-1-yl acetate, $1 \mathrm{~h}$

${ }^{1} \mathrm{H}$ NMR (300 MHz, $\mathrm{CDCl}_{3}$ )

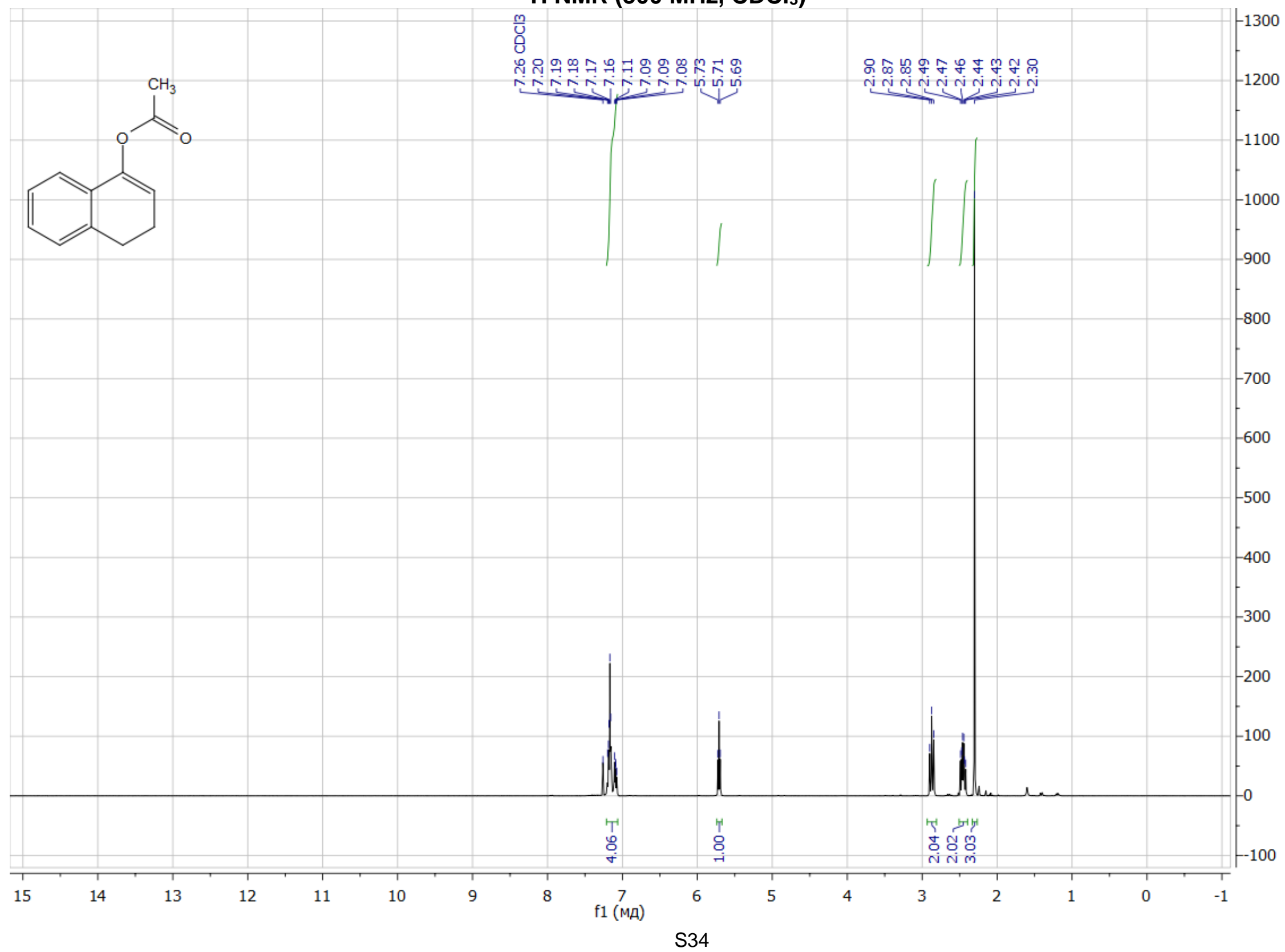


$\left.{ }^{13} \mathrm{C} \mathrm{NMR} \mathrm{(75} \mathrm{MHz,} \mathrm{CDCl}_{3}\right)$

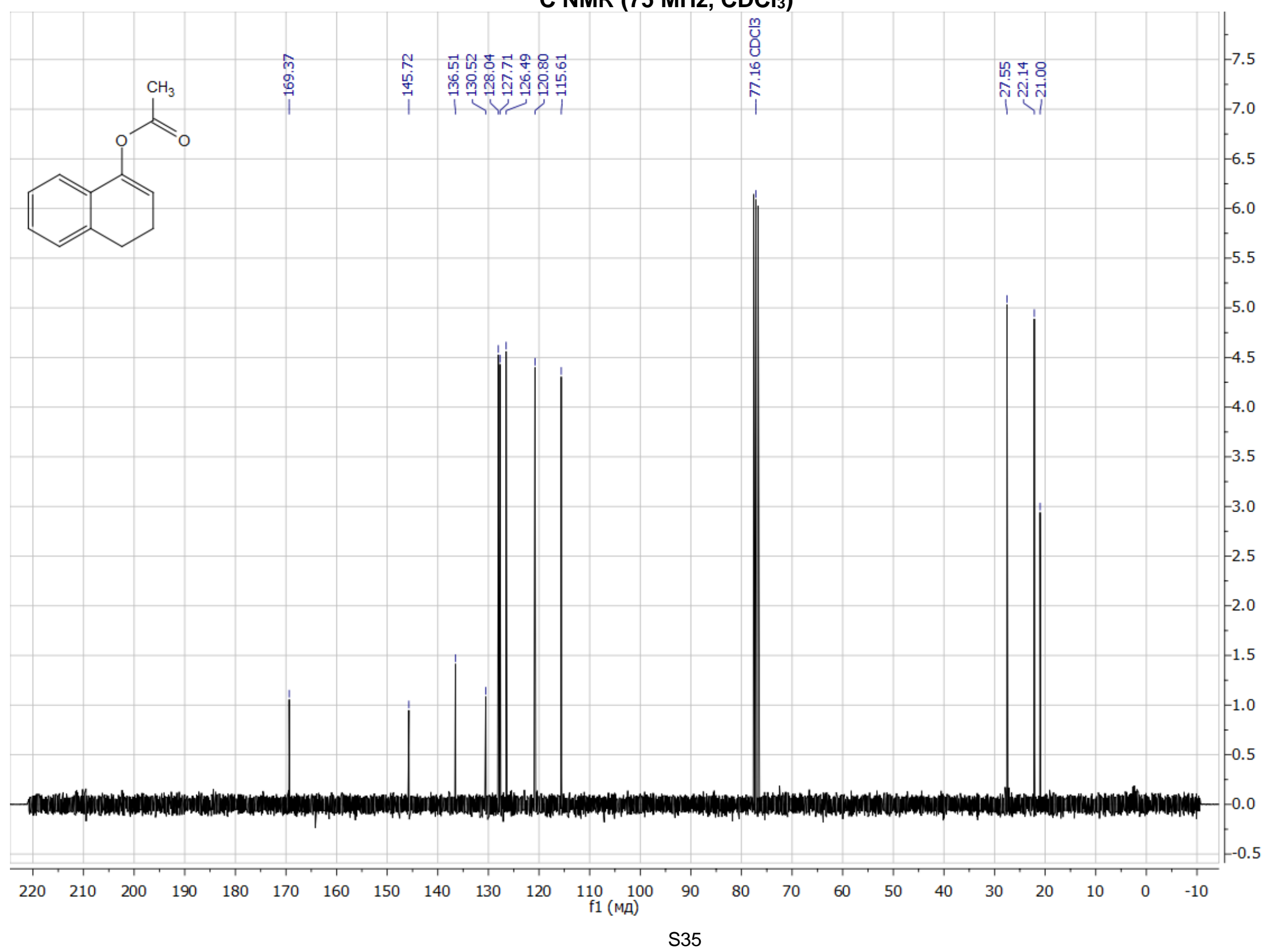


(Z)-1-Phenylprop-1-en-1-yl acetate, 1i

${ }^{1} \mathrm{H}$ NMR (300 MHz, $\mathrm{CDCl}_{3}$ )

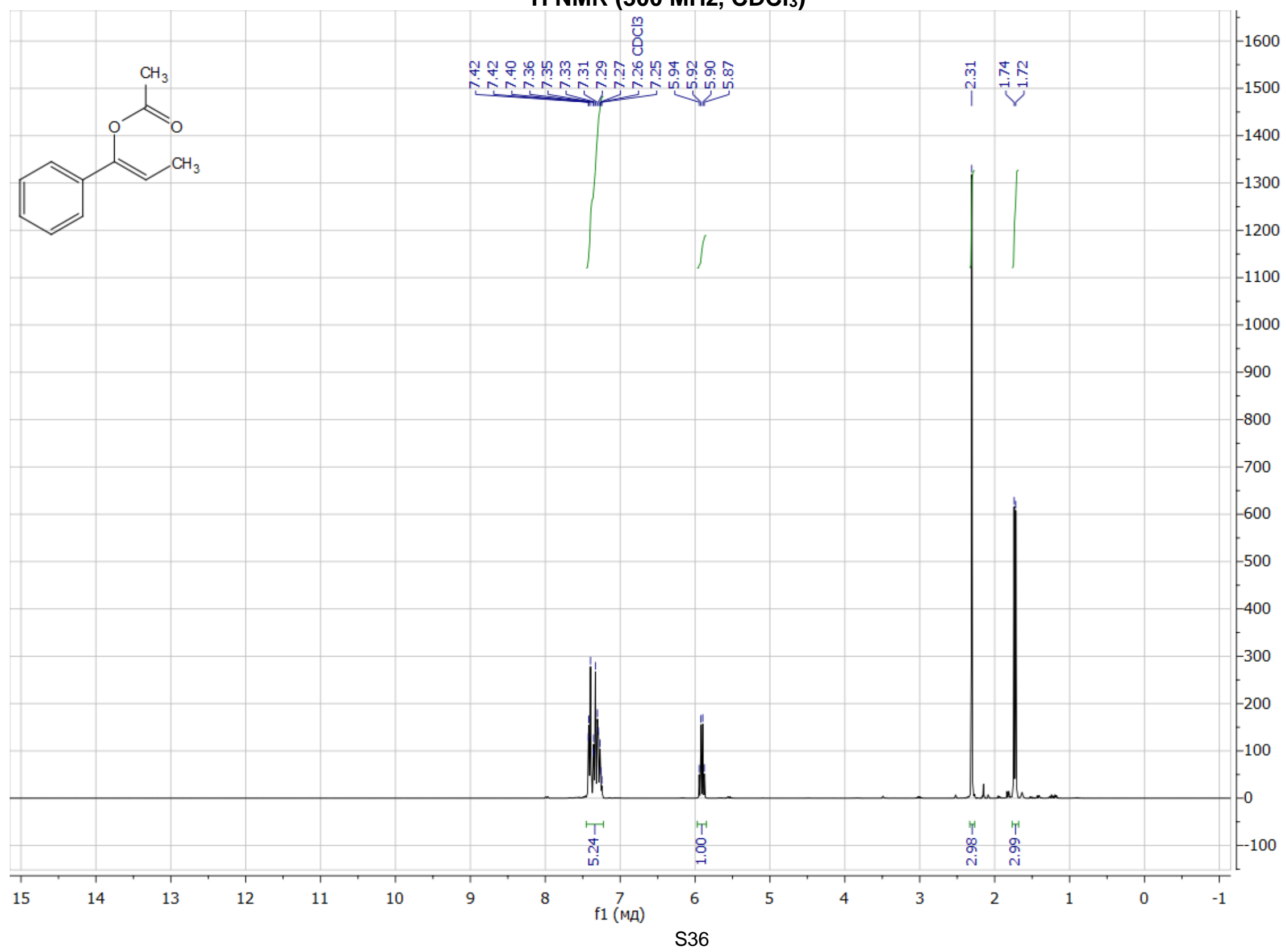


$\left.{ }^{13} \mathrm{C} \mathrm{NMR} \mathrm{(75} \mathrm{MHz,} \mathrm{CDCl}_{3}\right)$

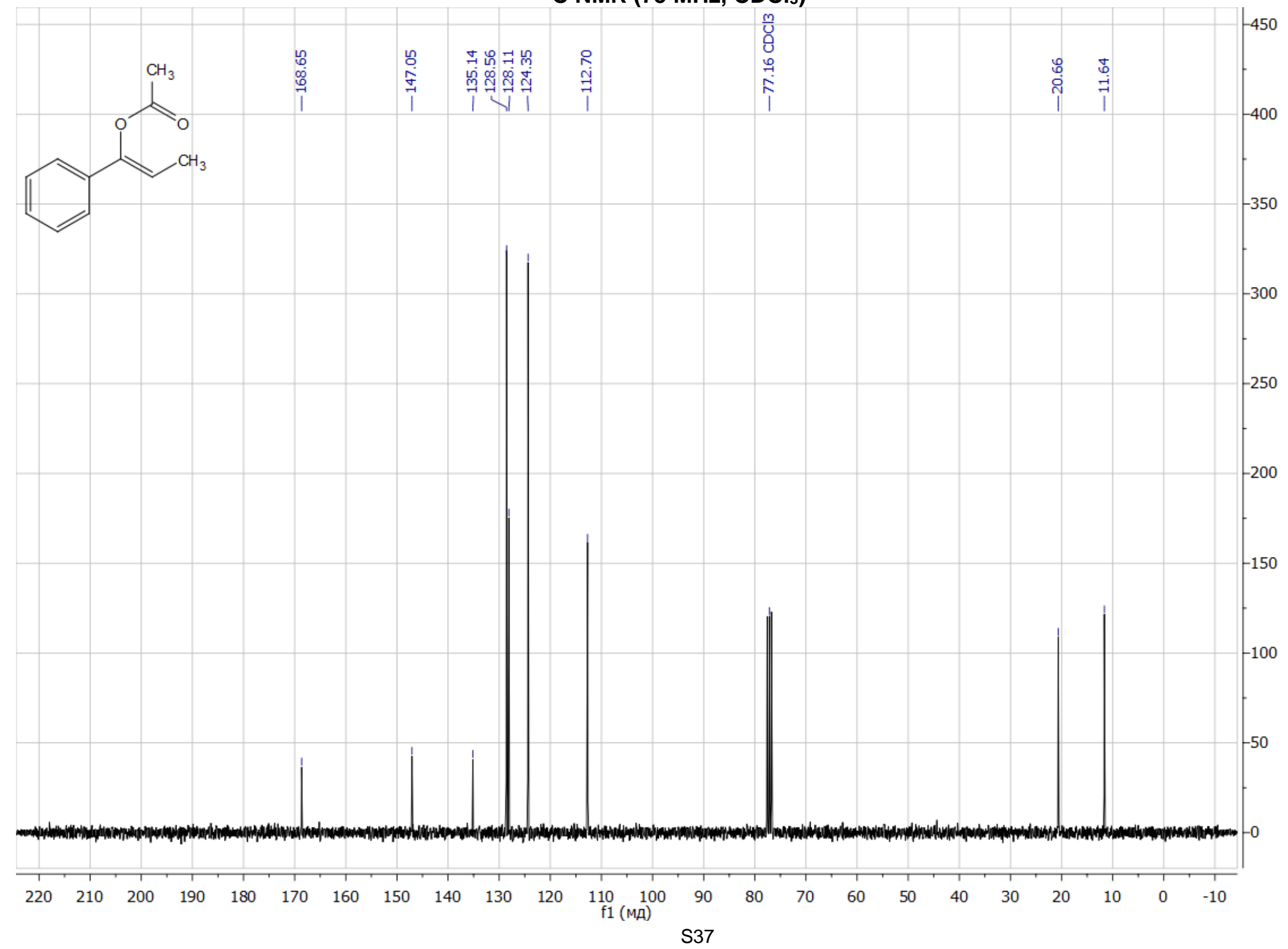


1-(Naphthalen-2-yl)vinyl acetate, 1j

${ }^{1} \mathrm{H}$ NMR (300 MHz, $\left.\mathrm{CDCl}_{3}\right)$

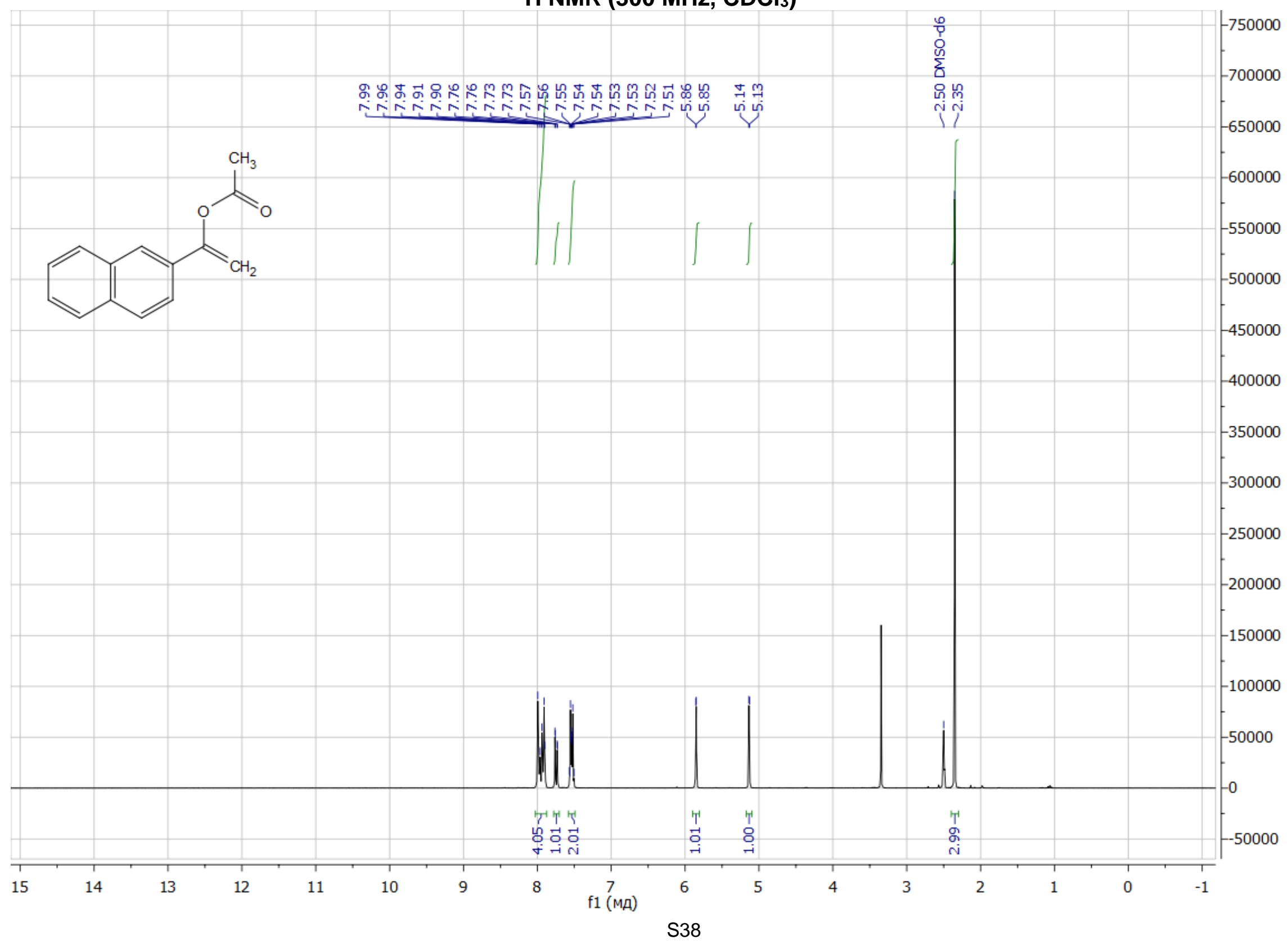


${ }^{13} \mathrm{C}$ NMR (75 MHz, $\left.\mathrm{CDCl}_{3}\right)$

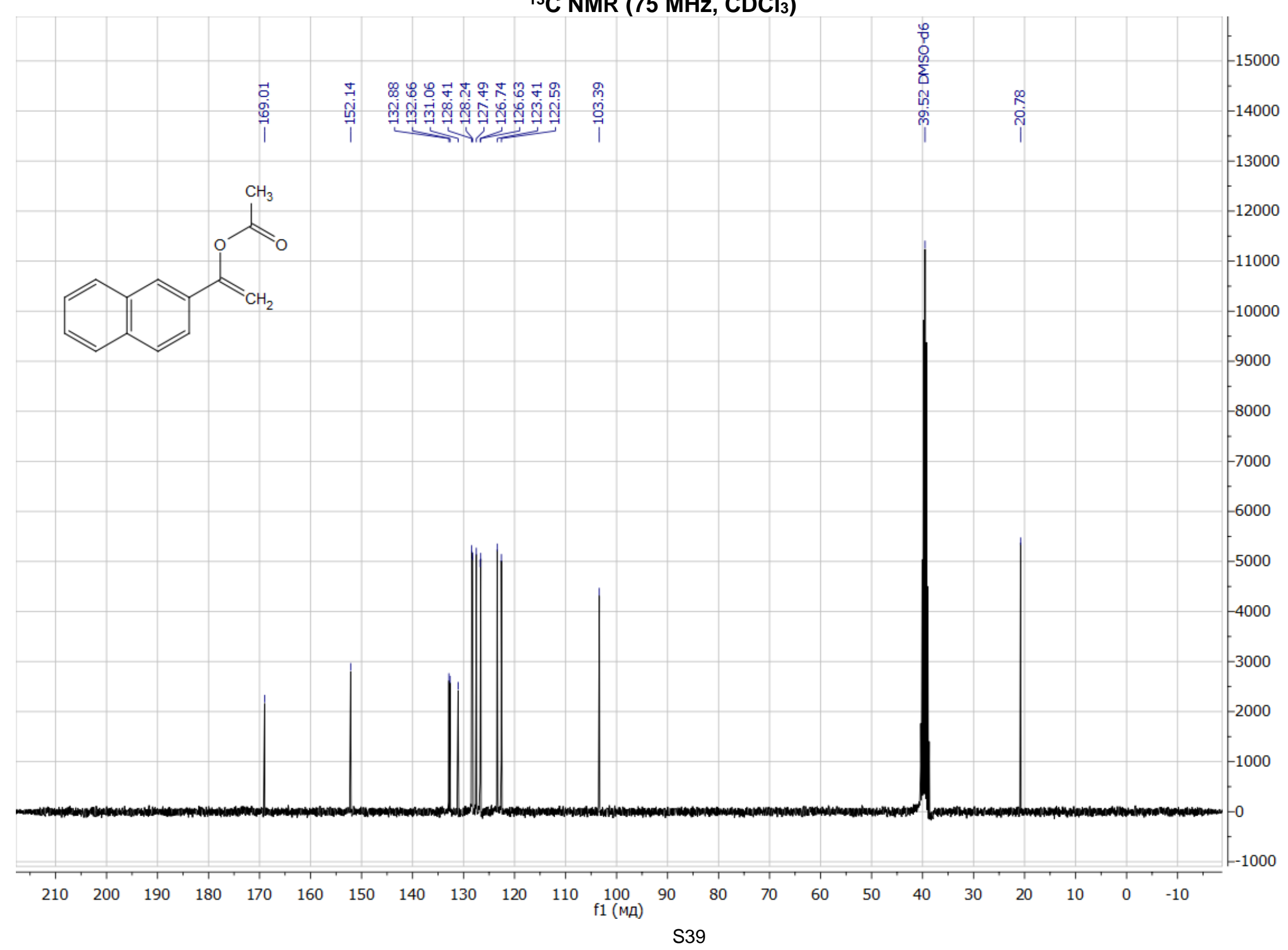


Cyclopent-1-en-1-yl acetate, $1 \mathrm{k}$

${ }^{1} \mathrm{H}$ NMR (300 MHz, $\left.\mathrm{CDCl}_{3}\right)$

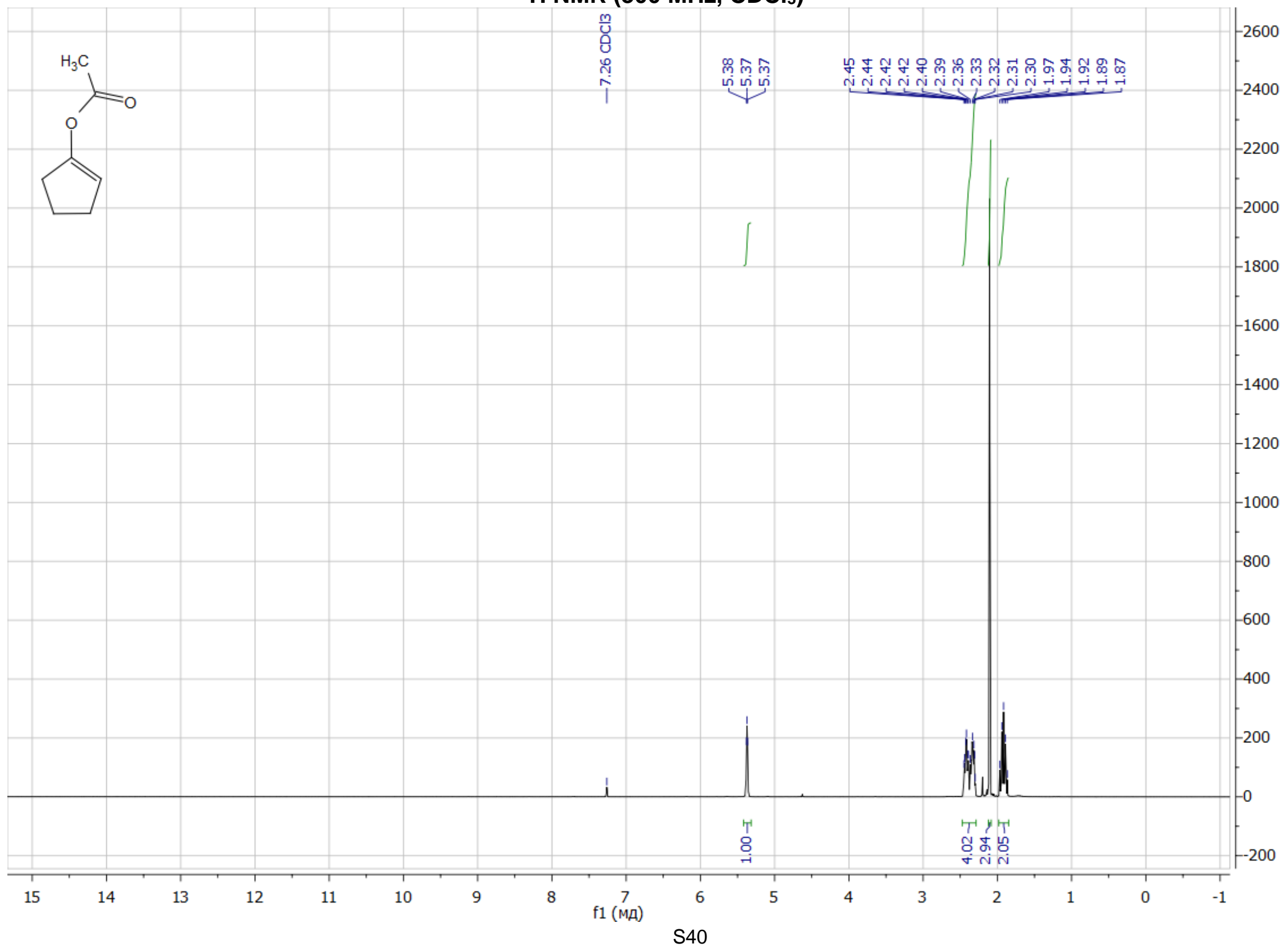


$\left.{ }^{13} \mathrm{C} \mathrm{NMR} \mathrm{(75} \mathrm{MHz,} \mathrm{CDCl}_{3}\right)$

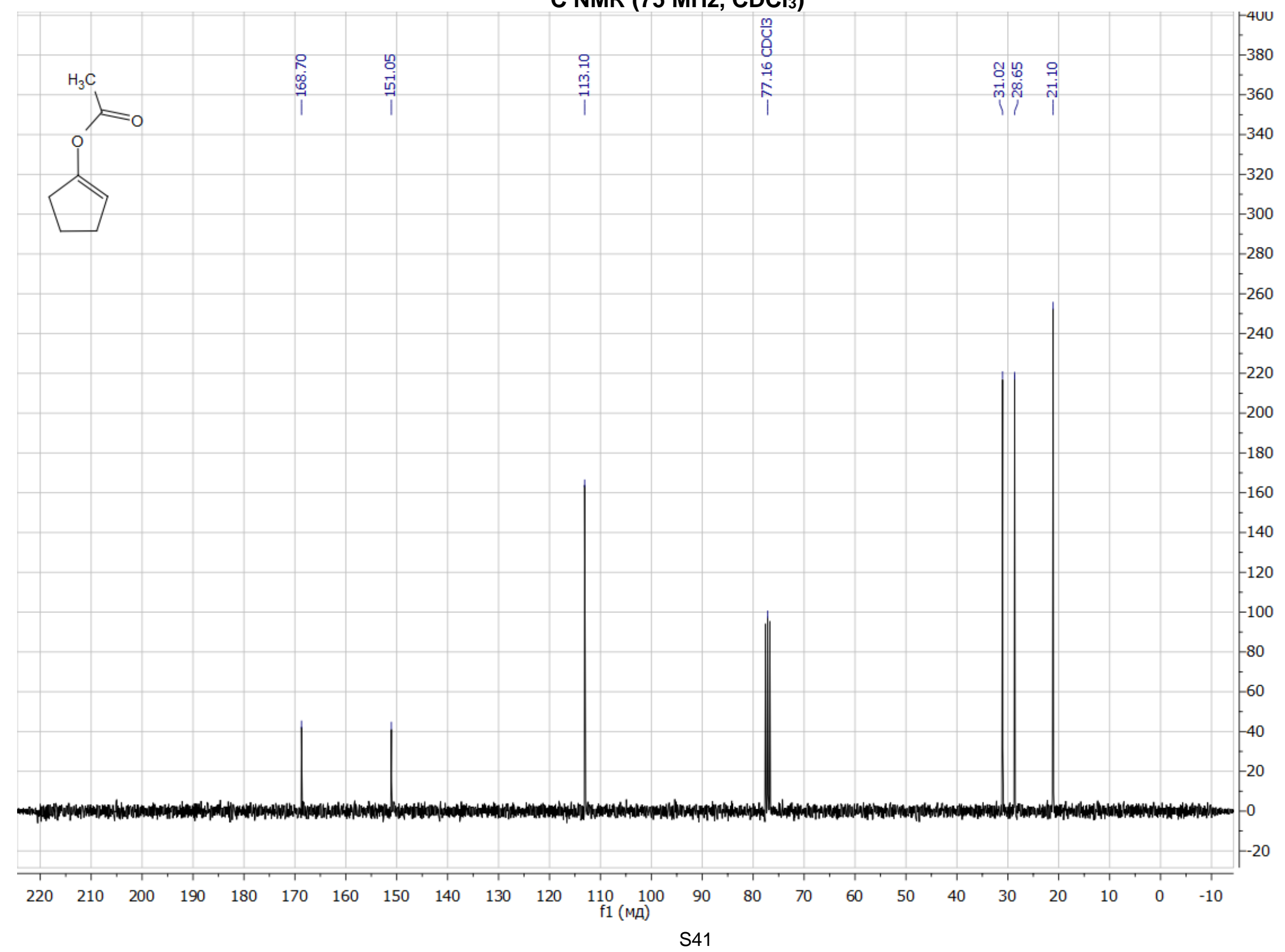


Cyclohex-1-en-1-yl acetate, 1 I

${ }^{1} \mathrm{H}$ NMR (300 MHz, $\left.\mathrm{CDCl}_{3}\right)$

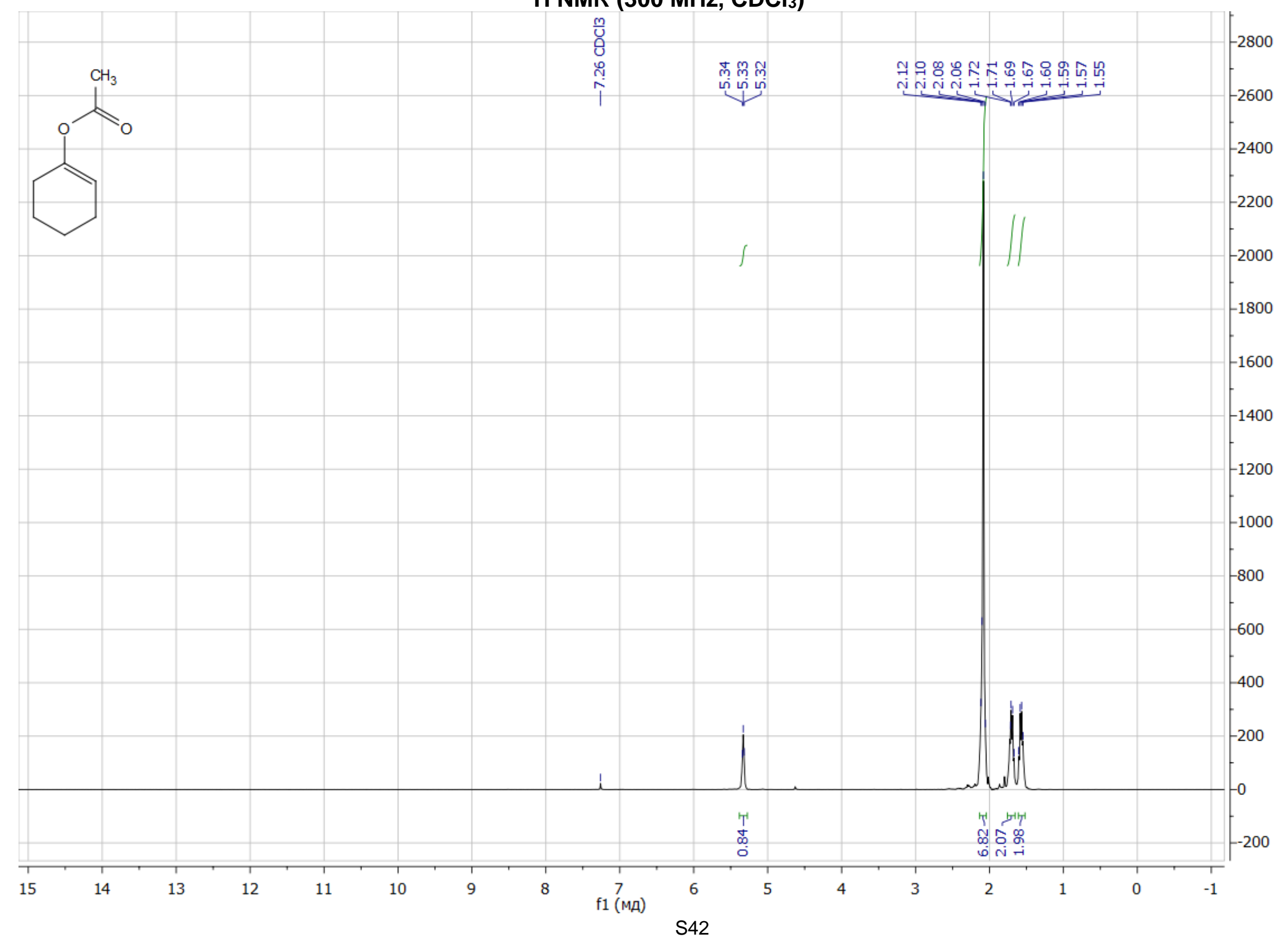


${ }^{13} \mathrm{C}$ NMR (75 MHz, $\left.\mathrm{CDCl}_{3}\right)$

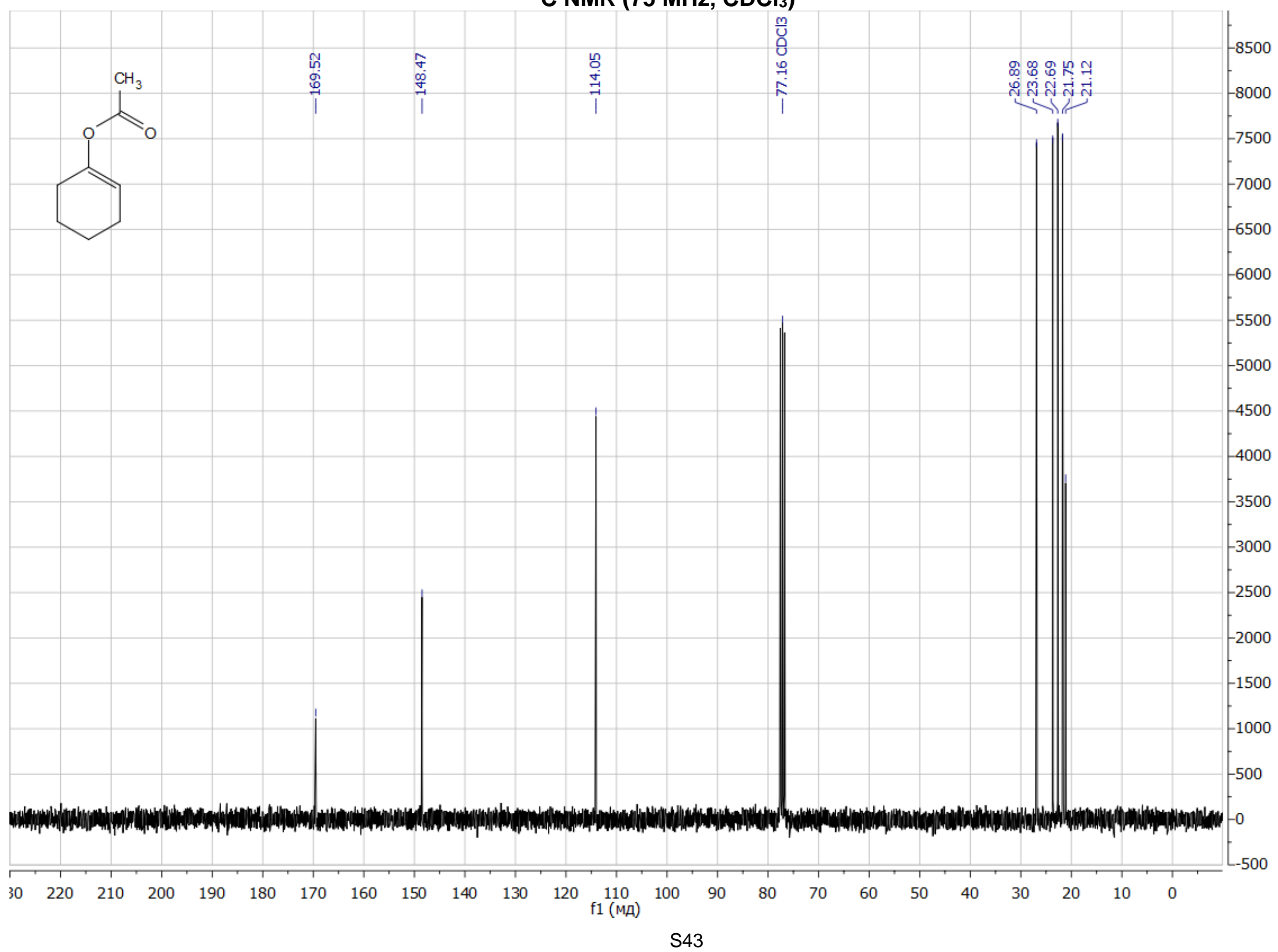


4-(Tert-butyl)cyclohex-1-en-1-yl acetate, $1 \mathrm{~m}$

${ }^{1} \mathrm{H}$ NMR (300 MHz, CDCl 3 )

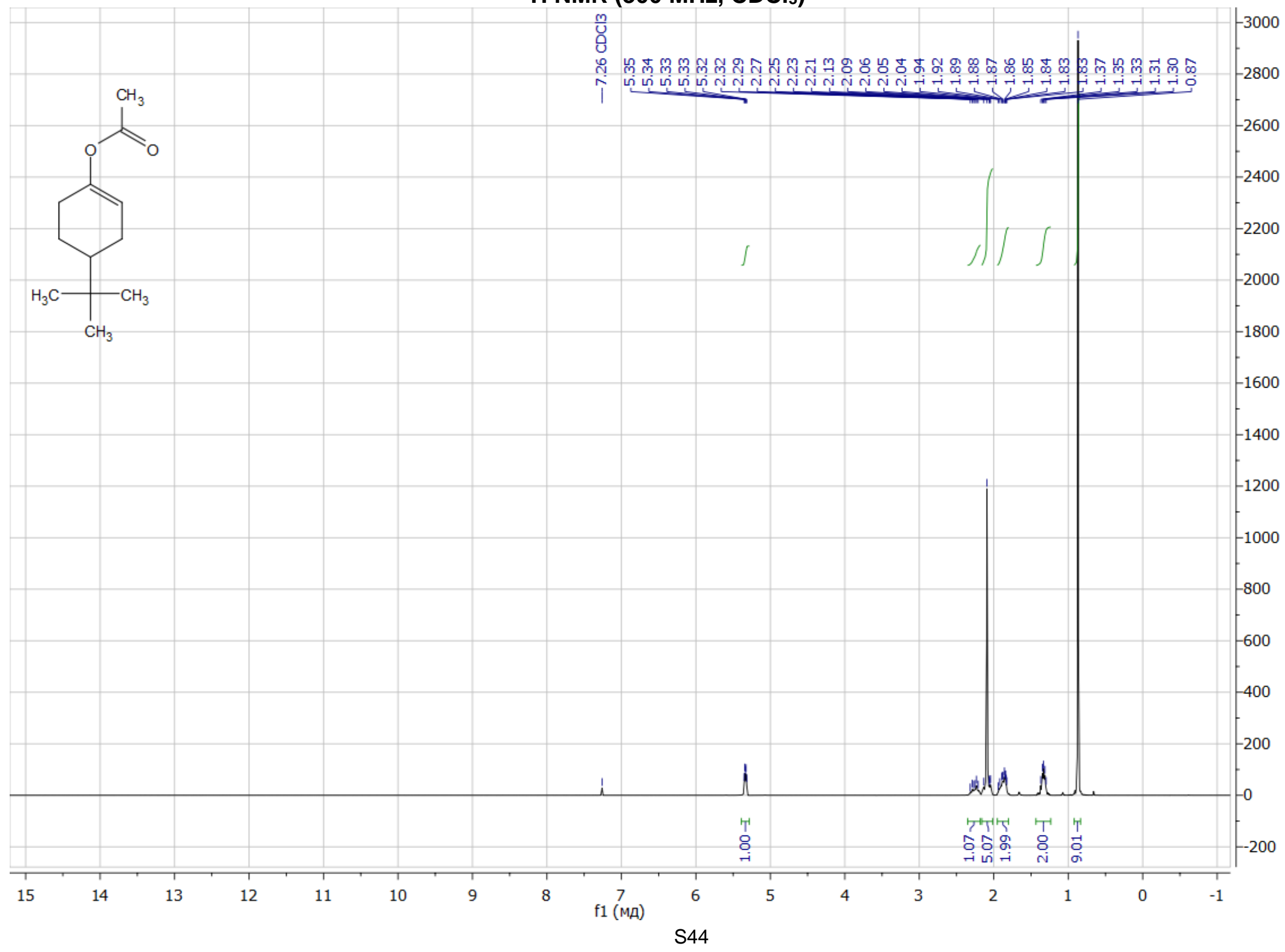


$\left.{ }^{13} \mathrm{C} \mathrm{NMR} \mathrm{(75} \mathrm{MHz,} \mathrm{CDCl}_{3}\right)$

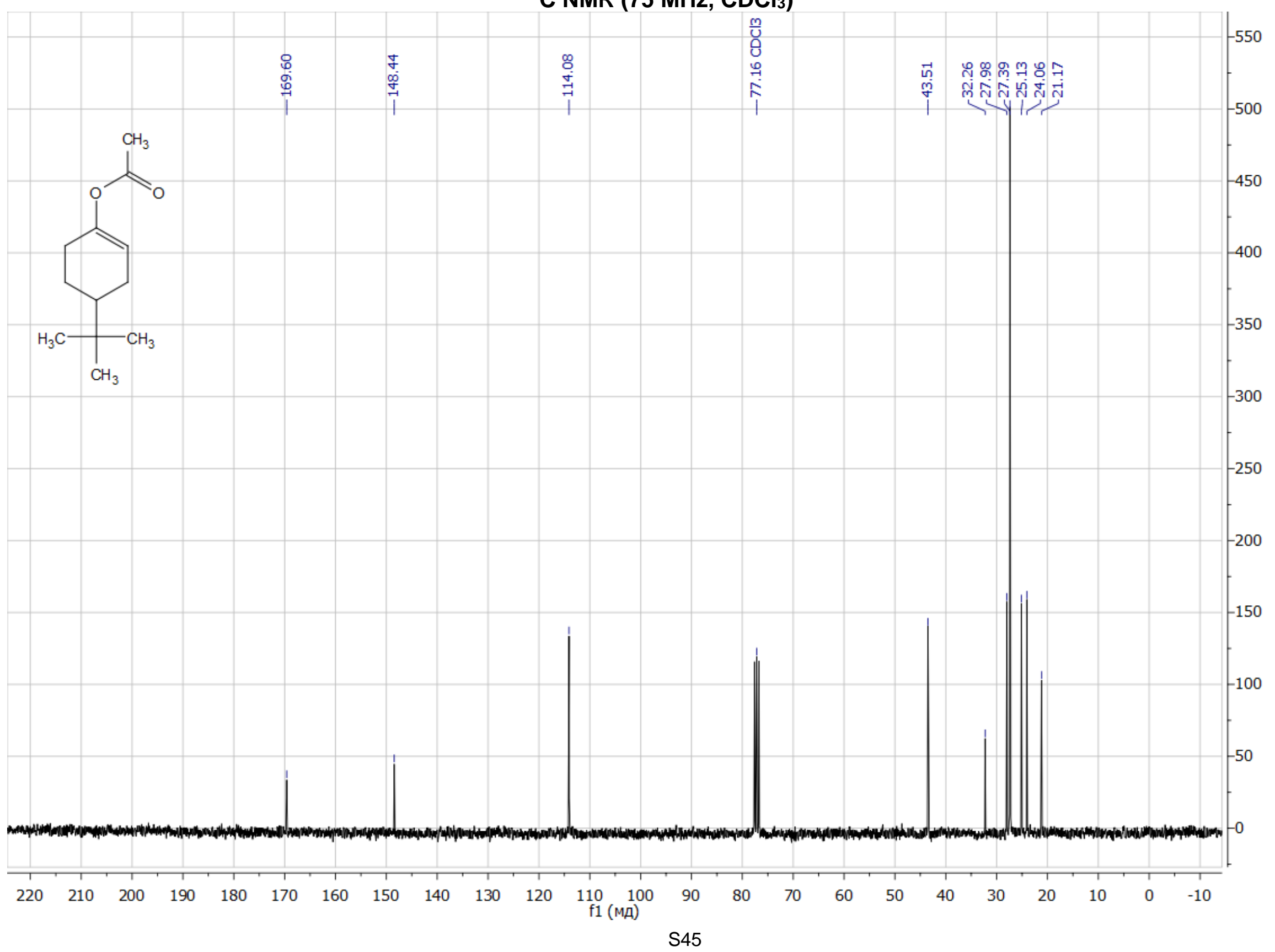


Cyclohept-1-en-1-yl acetate, 1n

${ }^{1} \mathrm{H}$ NMR (300 MHz, $\left.\mathrm{CDCl}_{3}\right)$

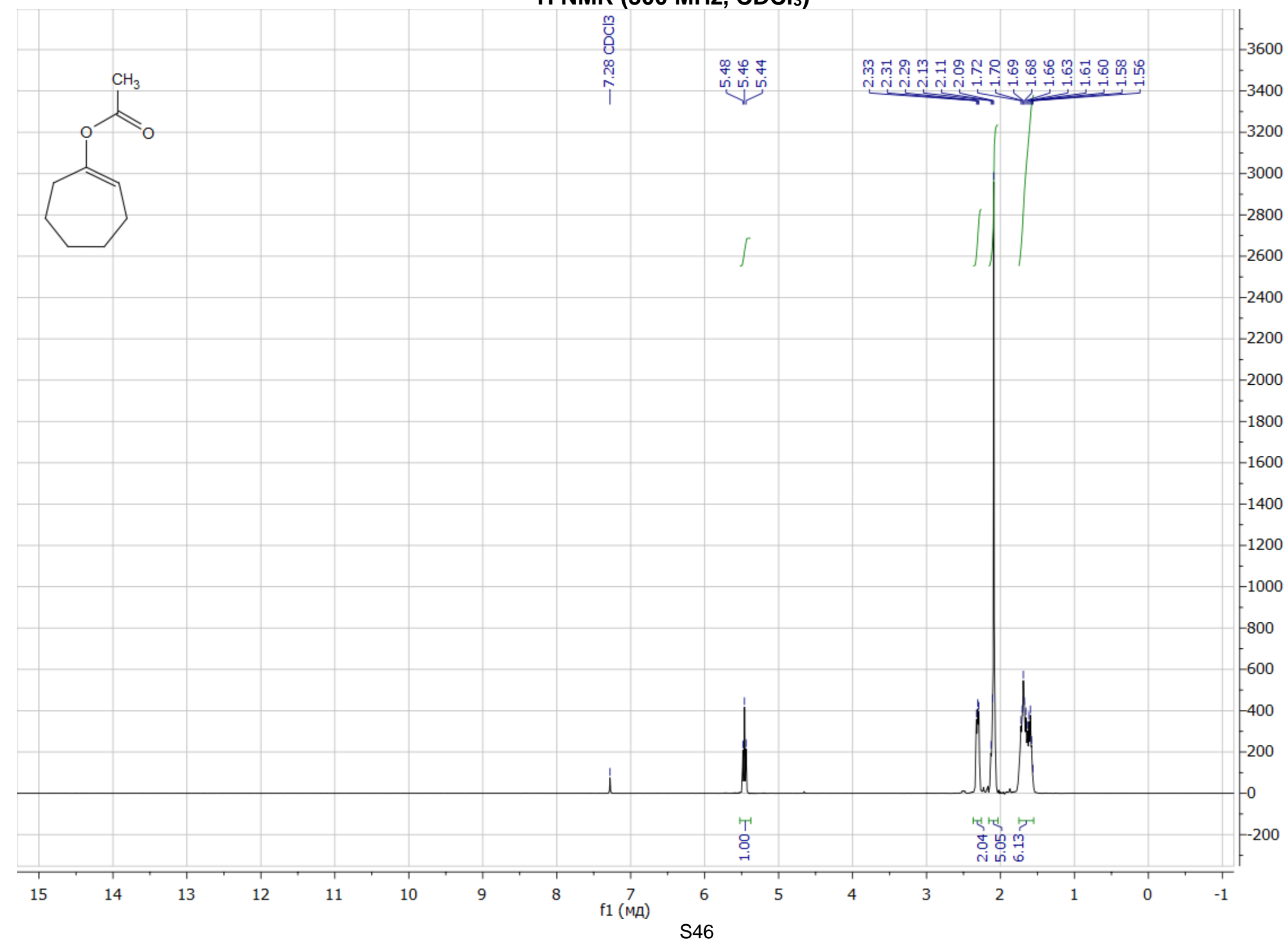


$\left.{ }^{13} \mathrm{C} \mathrm{NMR} \mathrm{(75} \mathrm{MHz,} \mathrm{CDCl}_{3}\right)$

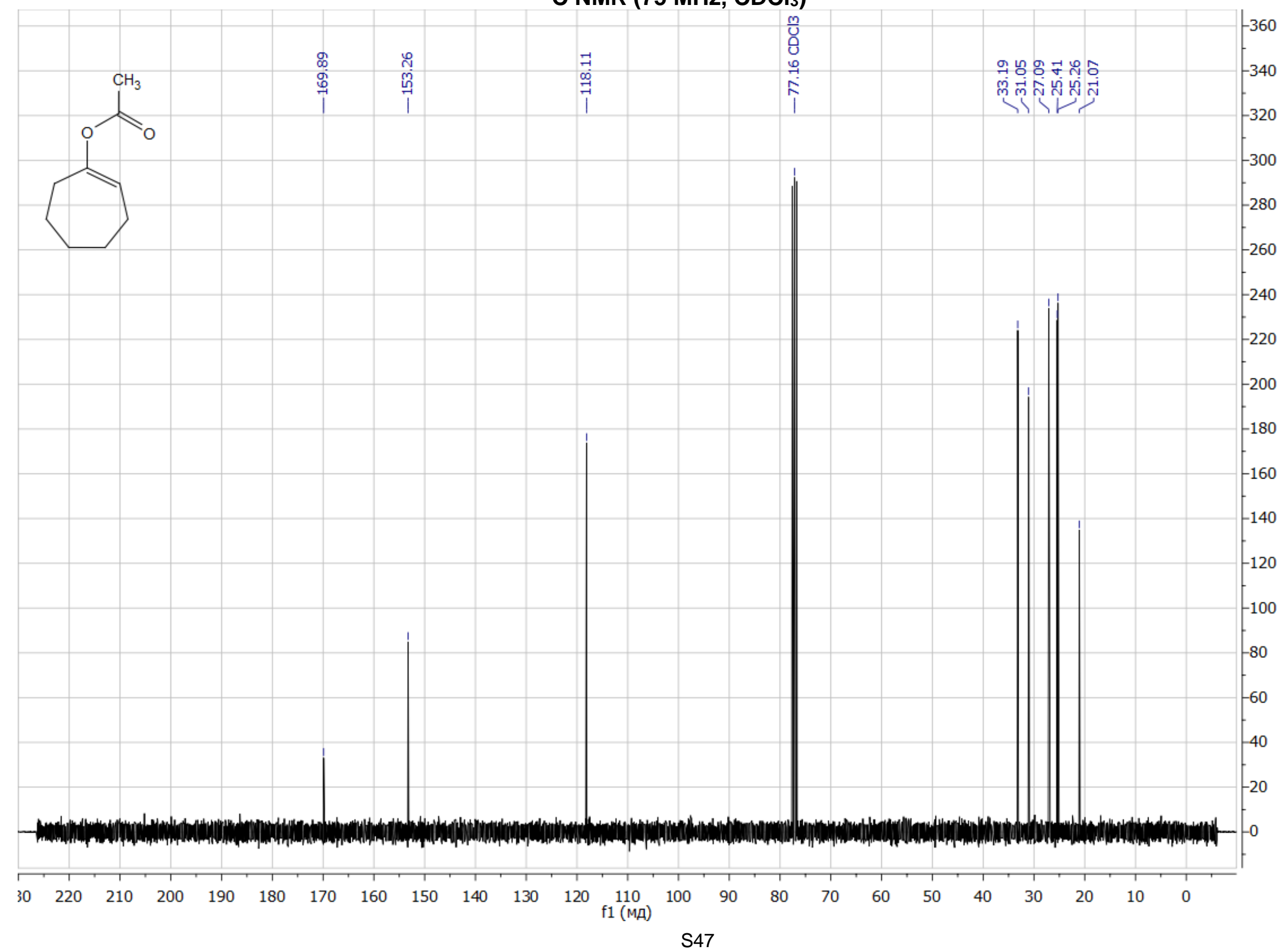


Cyclododec-1-en-1-yl acetate, 10

${ }^{1} \mathrm{H}$ NMR (300 MHz, $\left.\mathrm{CDCl}_{3}\right)$

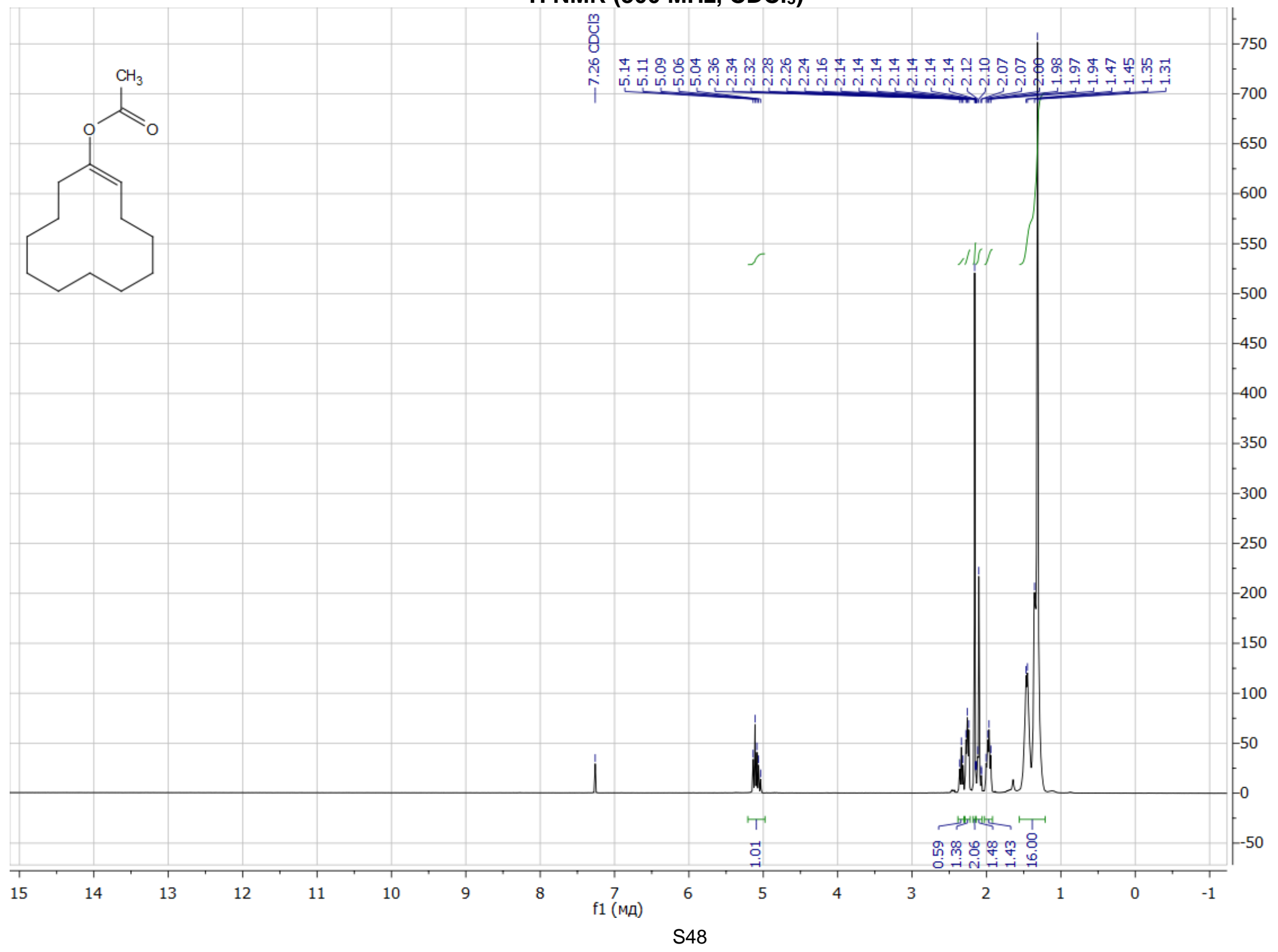


$\left.{ }^{13} \mathrm{C} \mathrm{NMR} \mathrm{(75} \mathrm{MHz,} \mathrm{CDCl}_{3}\right)$

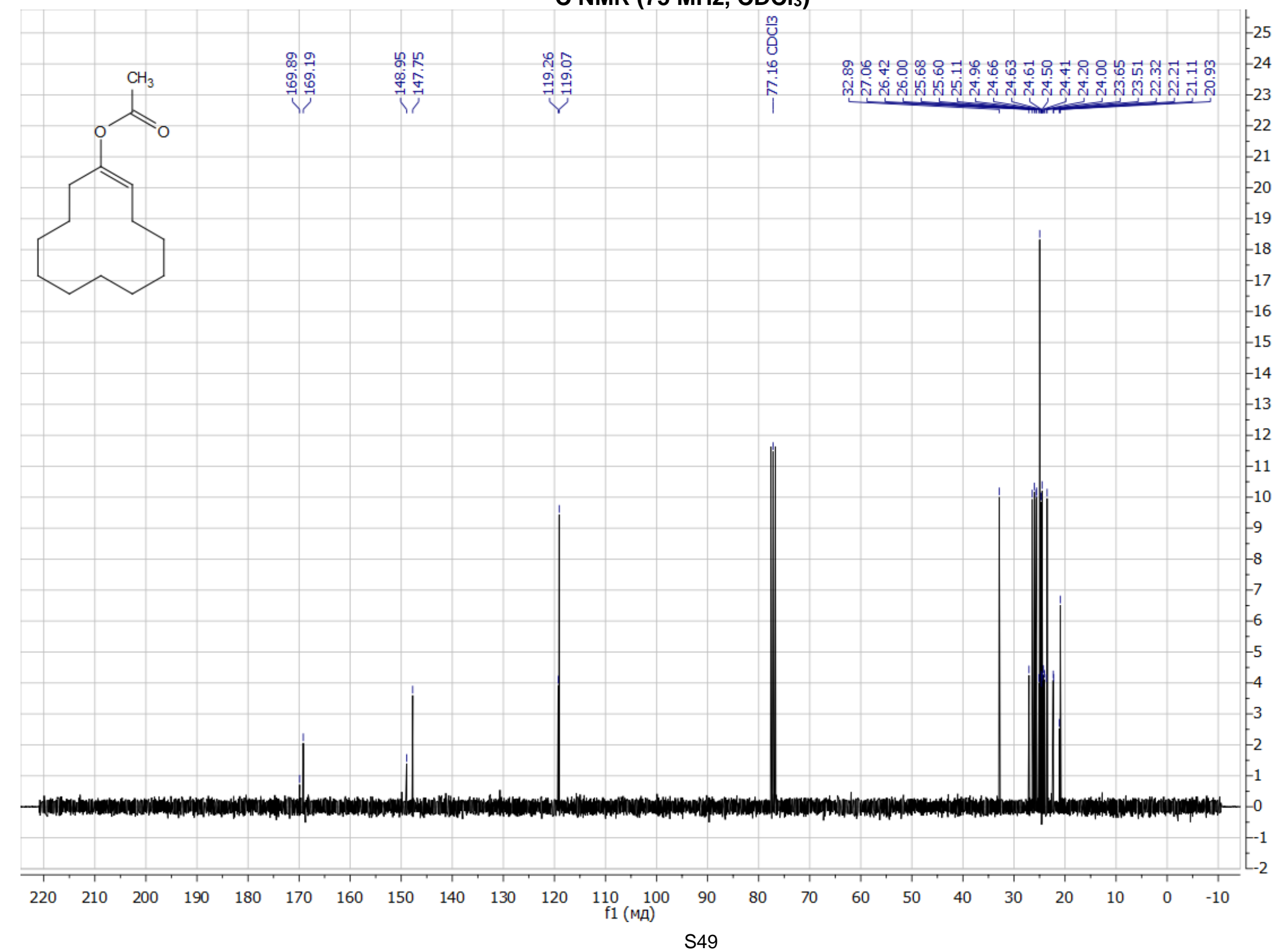


NMR spectra of obtained fluorinated ketones

3,3,3-Trifluoro-1-phenylpropan-1-one, 2a ${ }^{1} \mathrm{H}$ NMR (300 MHz, $\mathrm{CDCl}_{3}$ )

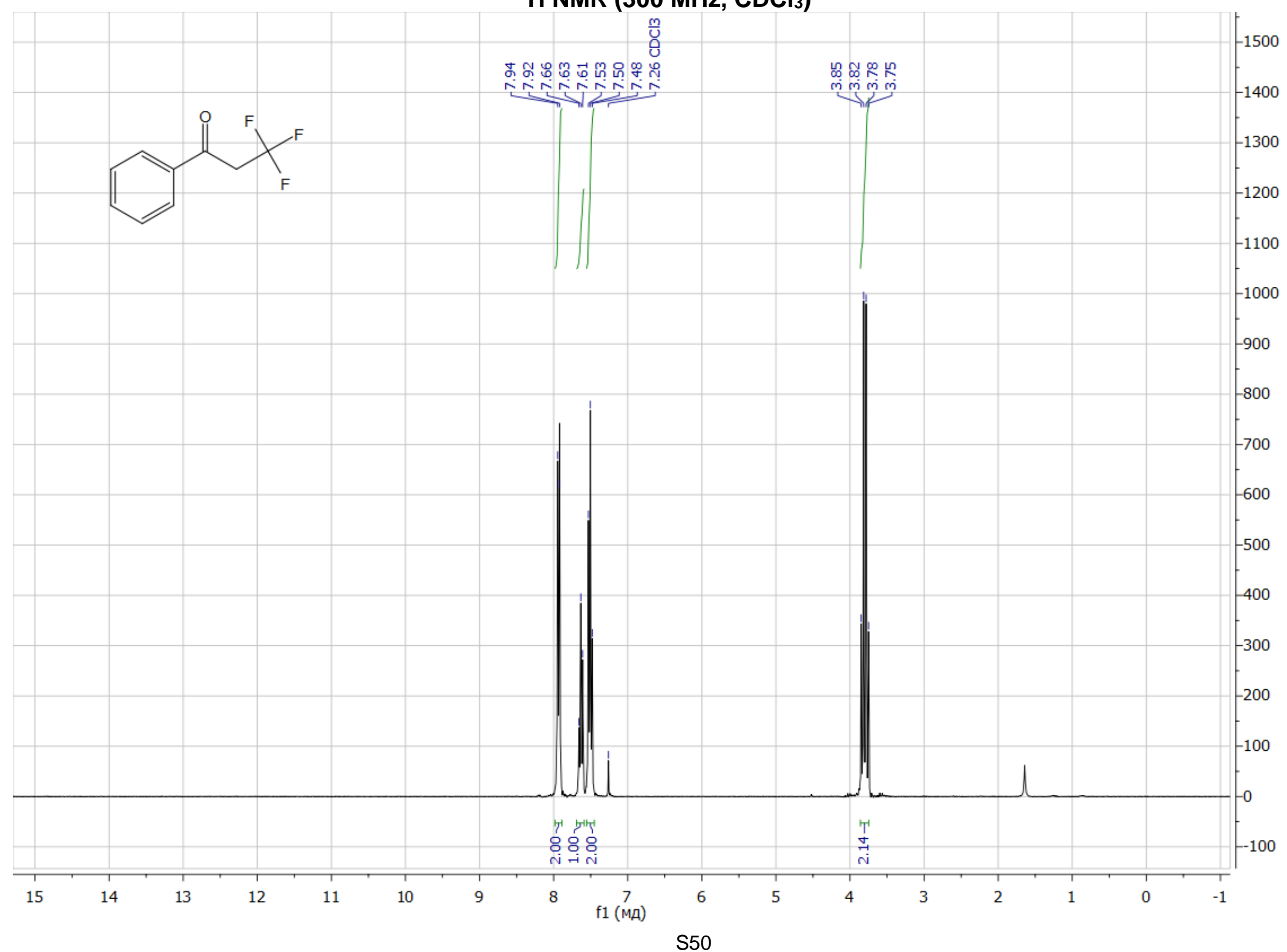


${ }^{13} \mathrm{C}$ NMR (75 MHz, $\left.\mathrm{CDCl}_{3}\right)$

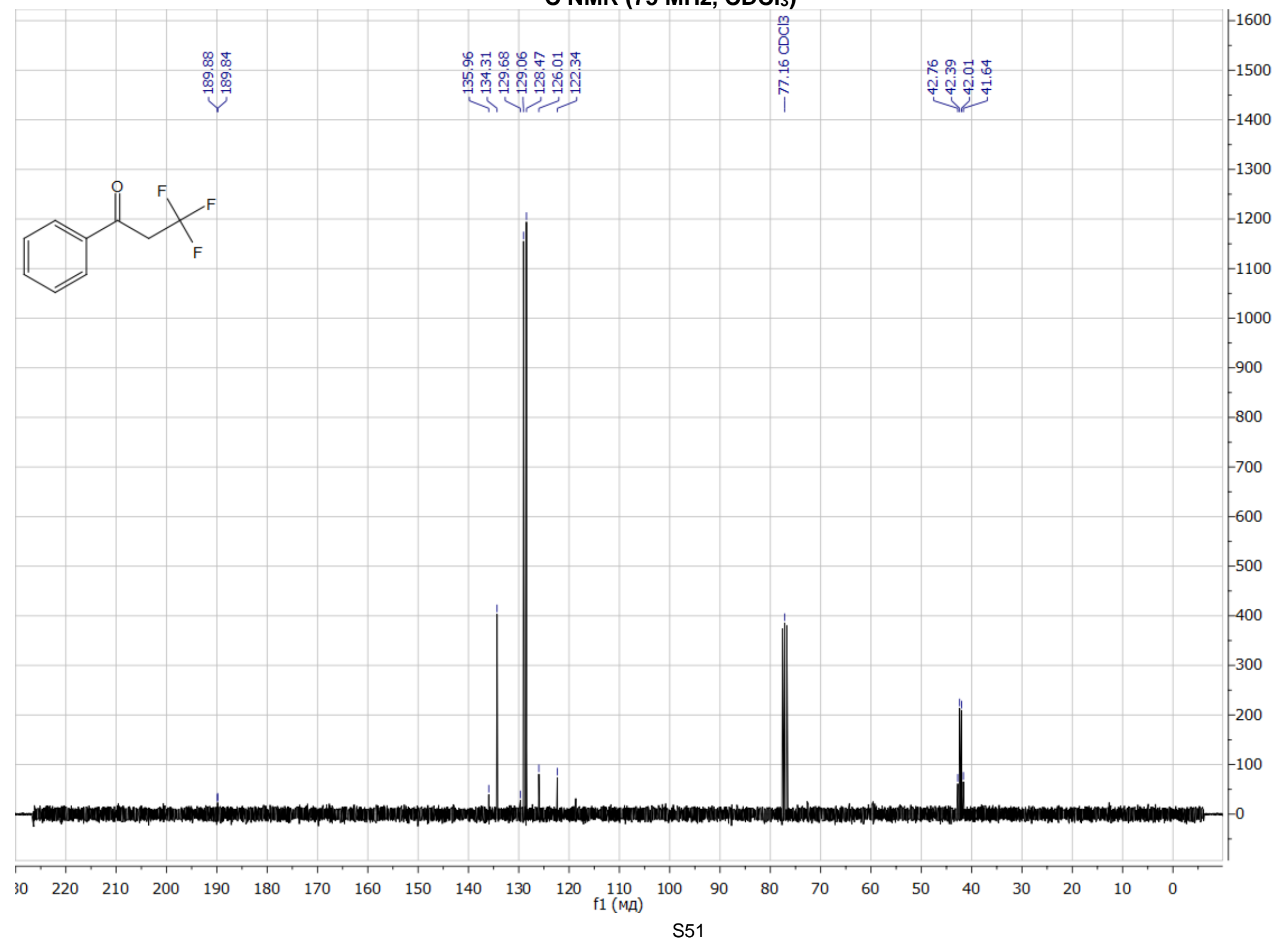


${ }^{19} \mathrm{~F}$ NMR (282 $\mathrm{MHz}, \mathrm{CDCl}_{3}$ )

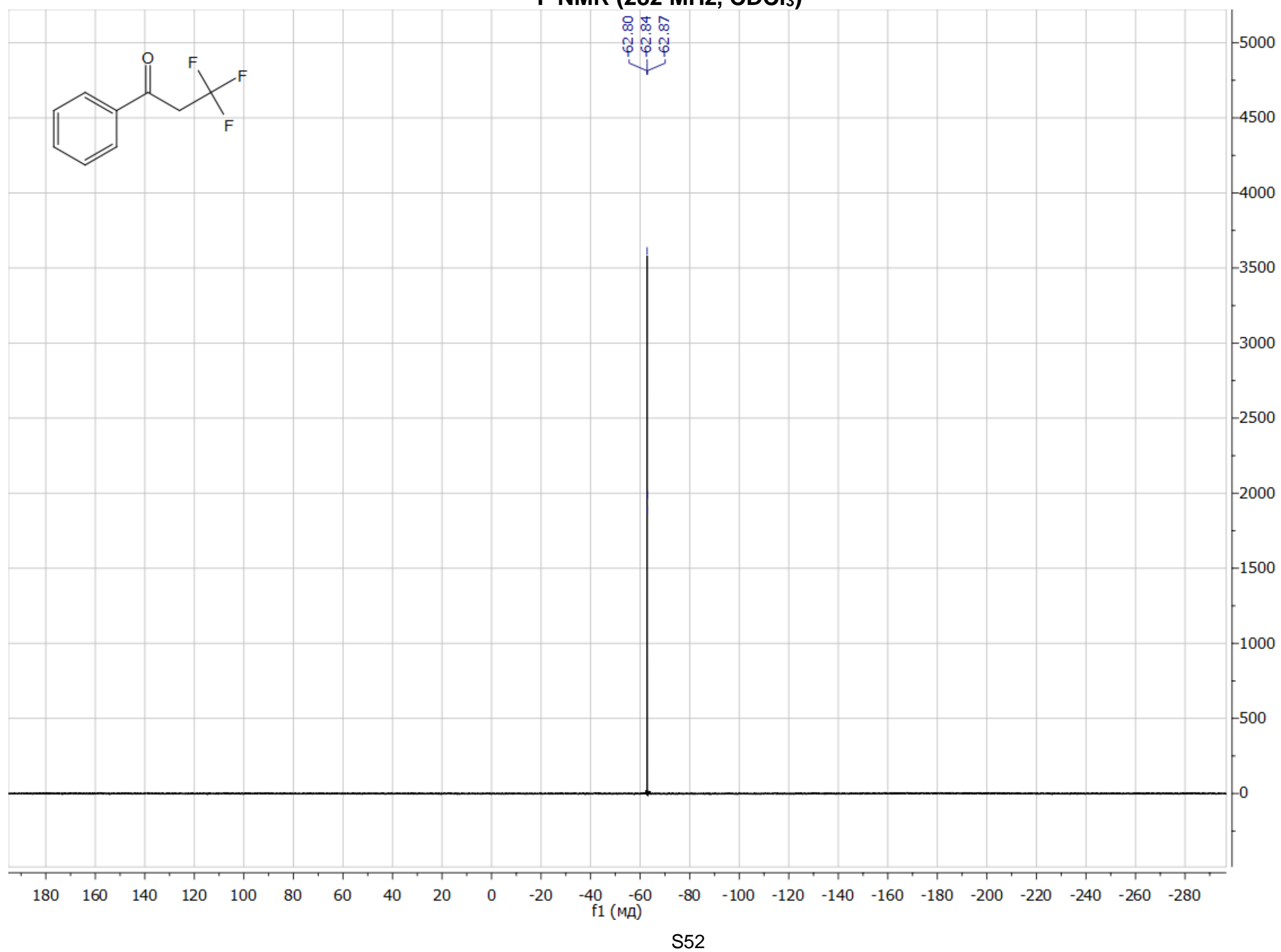


3,3,3-Trifluoro-1-(p-tolyl)propan-1-one, 2b

${ }^{1} \mathrm{H}$ NMR (300 MHz, CDCl ${ }_{3}$ )

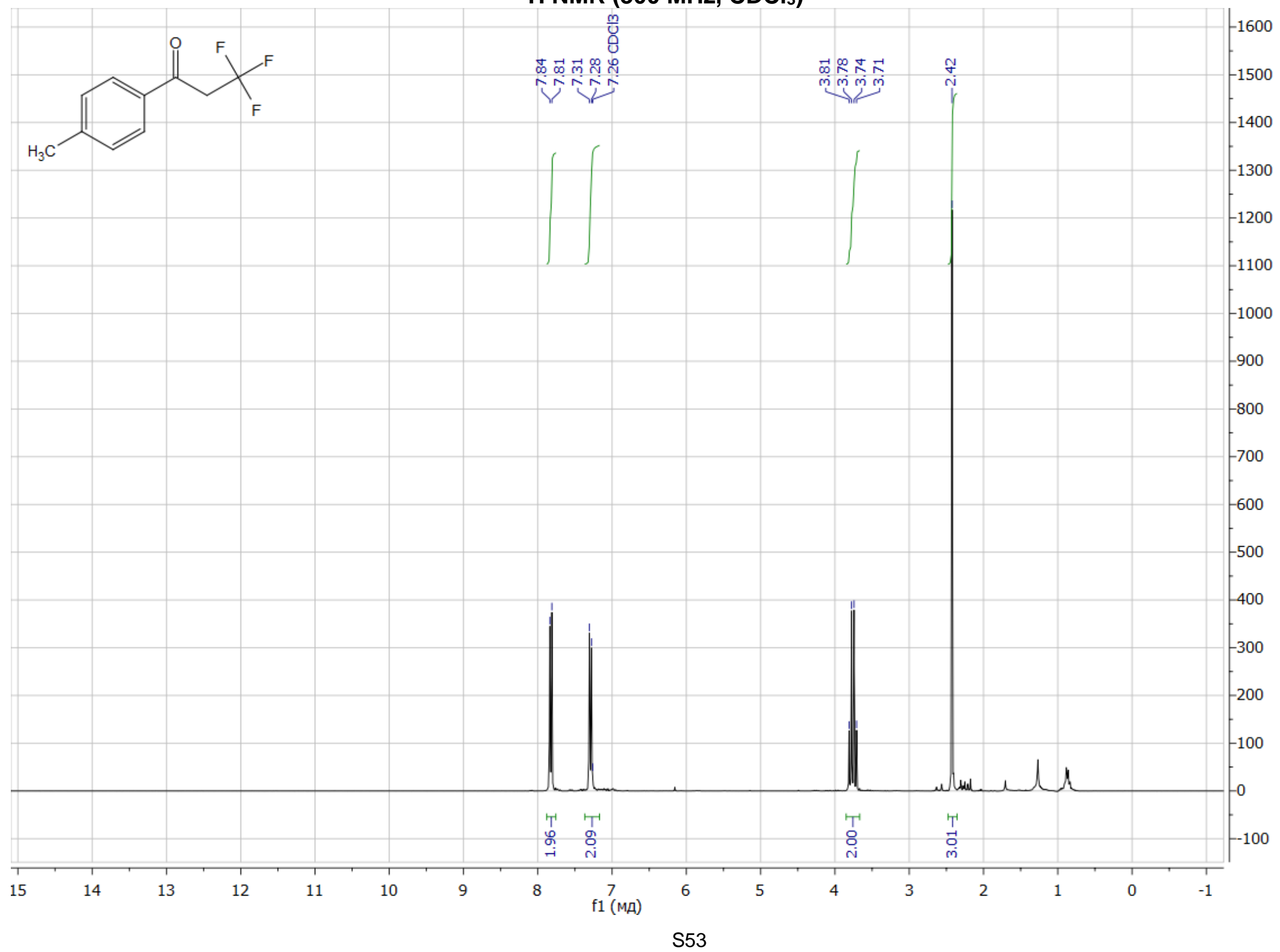


$\left.{ }^{13} \mathrm{C} \mathrm{NMR} \mathrm{(75} \mathrm{MHz,} \mathrm{CDCl}_{3}\right)$

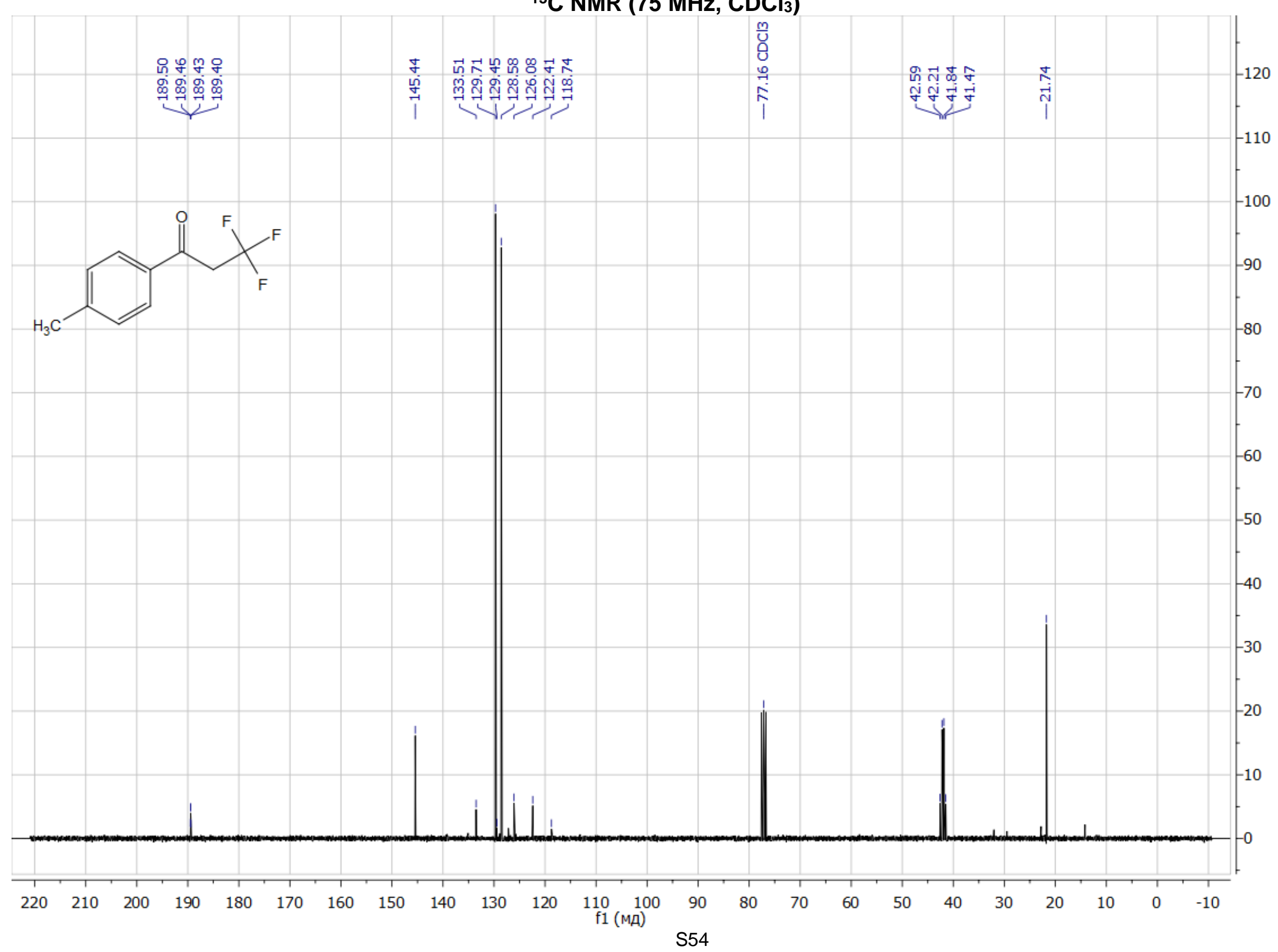


${ }^{19} \mathrm{~F}$ NMR (282 $\left.\mathrm{MHz}, \mathrm{CDCl}_{3}\right)$

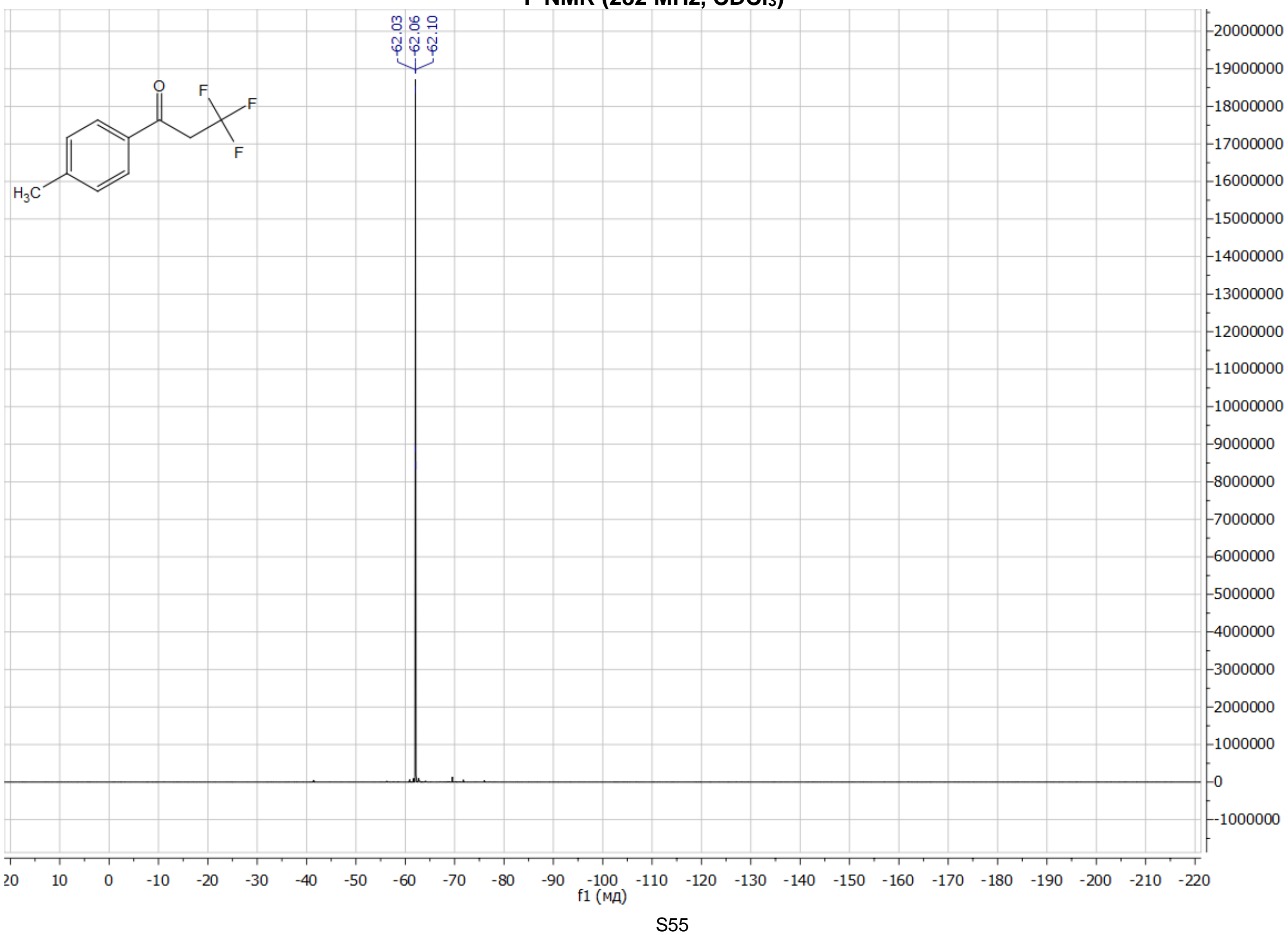


3,3,3-Trifluoro-1-(4-methoxyphenyl)propan-1-one, 2c ${ }^{1} \mathrm{H}$ NMR (300 MHz, CDCl 3 )

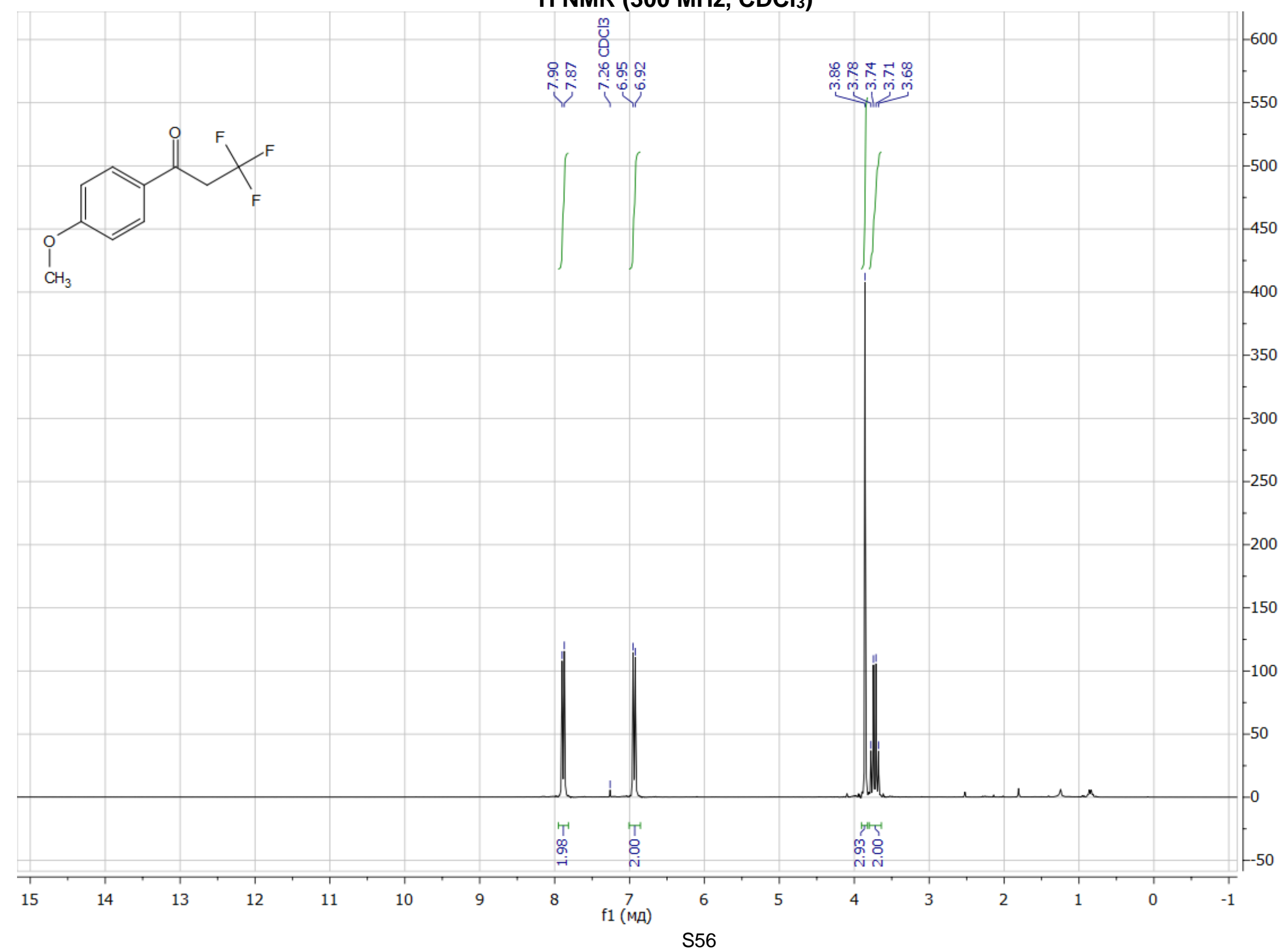


${ }^{13} \mathrm{C}$ NMR (75 MHz, $\left.\mathrm{CDCl}_{3}\right)$

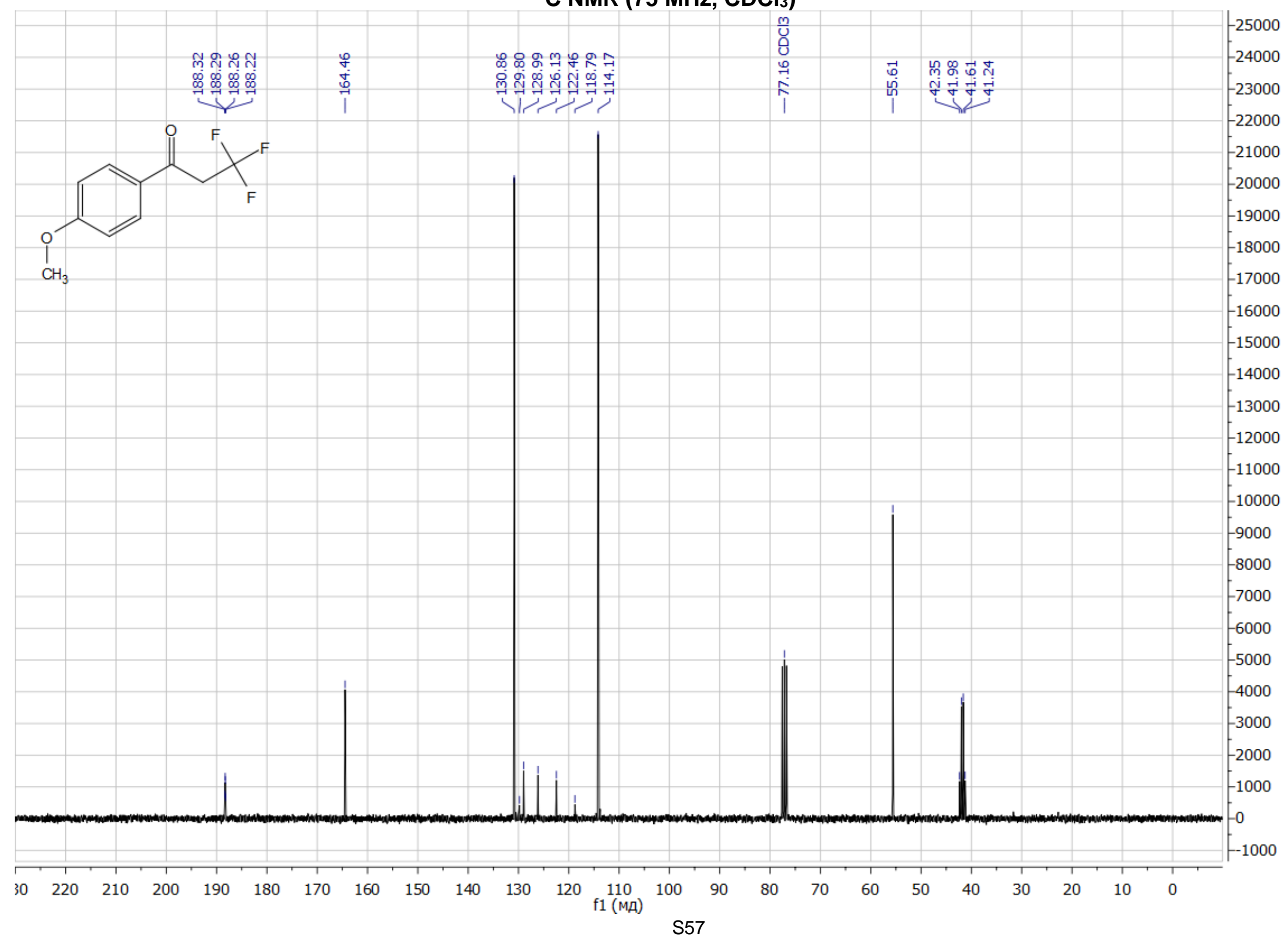


${ }^{19} \mathrm{~F}$ NMR (282 $\left.\mathrm{MHz}, \mathrm{CDCl}_{3}\right)$

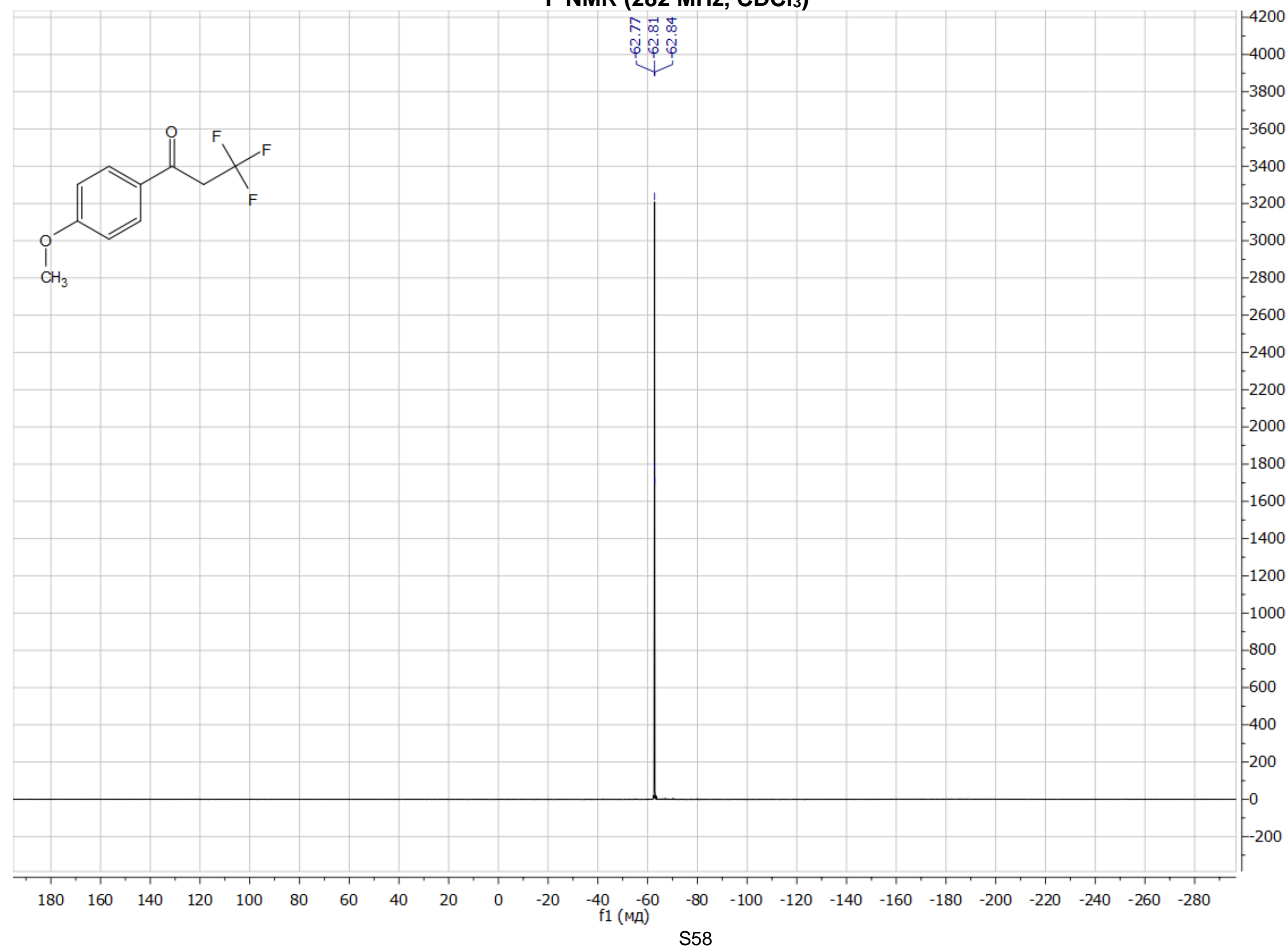


1-(4-Chlorophenyl)-3,3,3-trifluoropropan-1-one, 2d

${ }^{1} \mathrm{H}$ NMR (300 MHz, CDCl 3 )

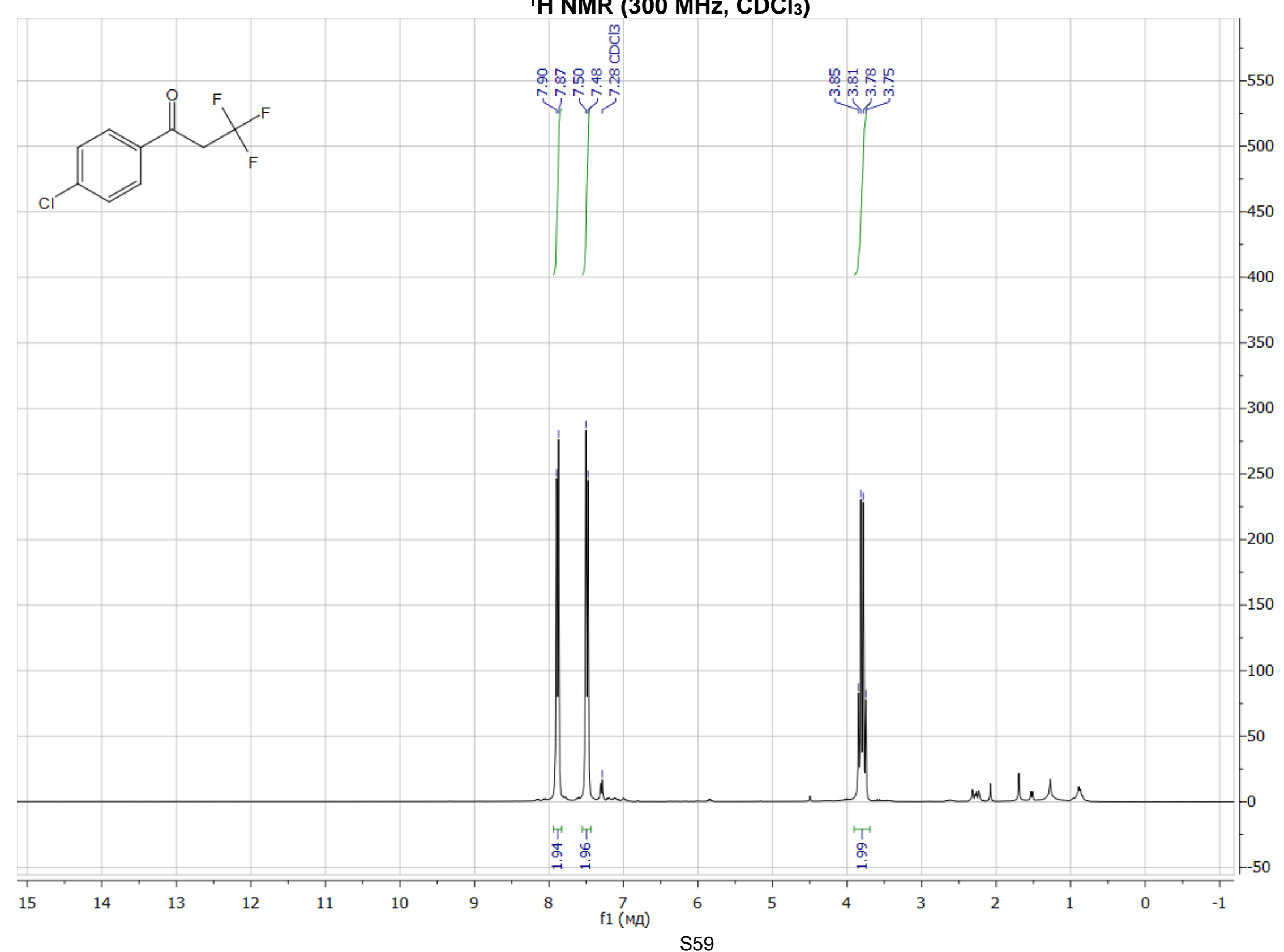


$\left.{ }^{13} \mathrm{C} \mathrm{NMR} \mathrm{(75} \mathrm{MHz,} \mathrm{CDCl}_{3}\right)$

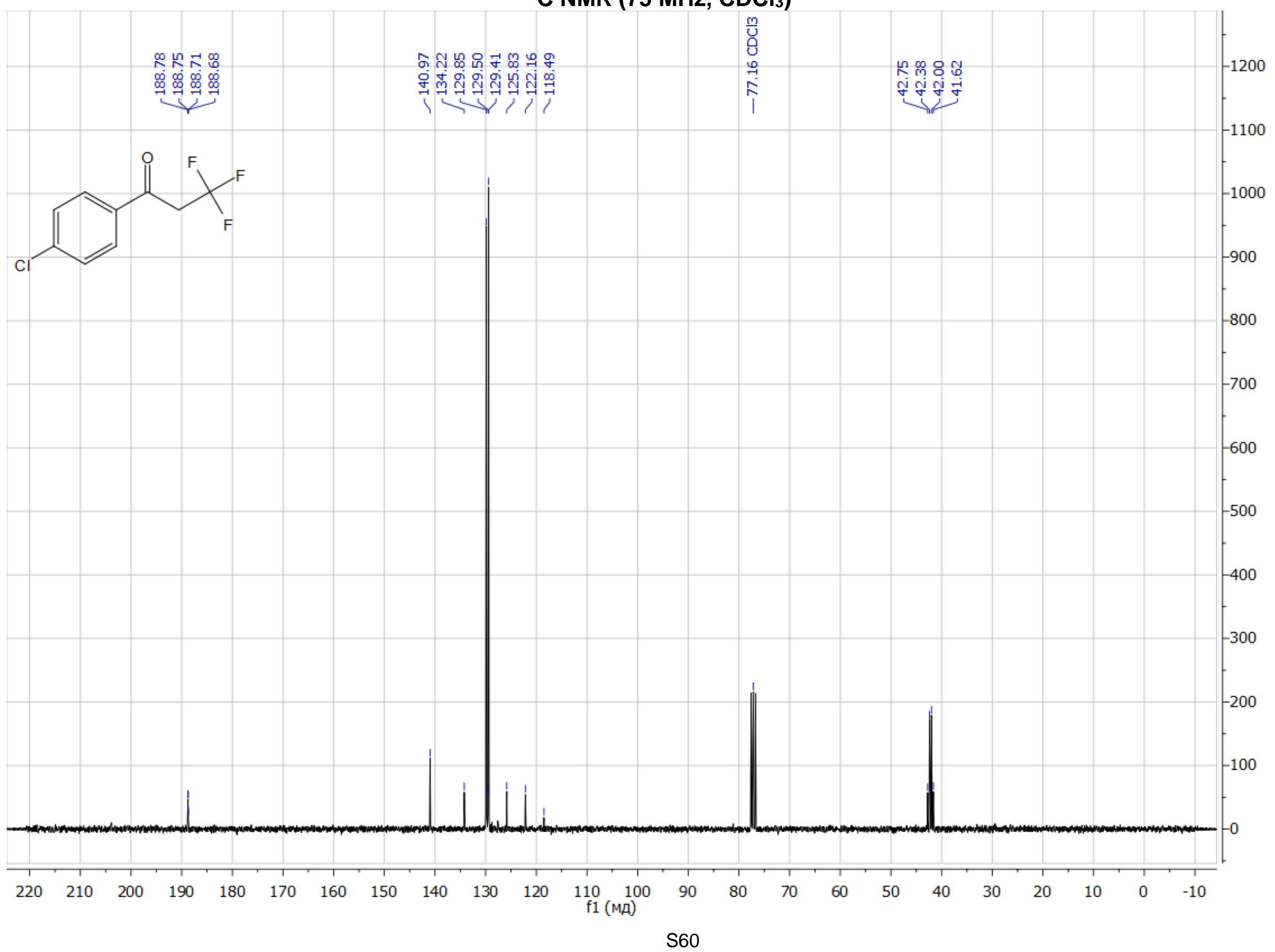


${ }^{19} \mathrm{~F}$ NMR (282 $\left.\mathrm{MHz}, \mathrm{CDCl}_{3}\right)$

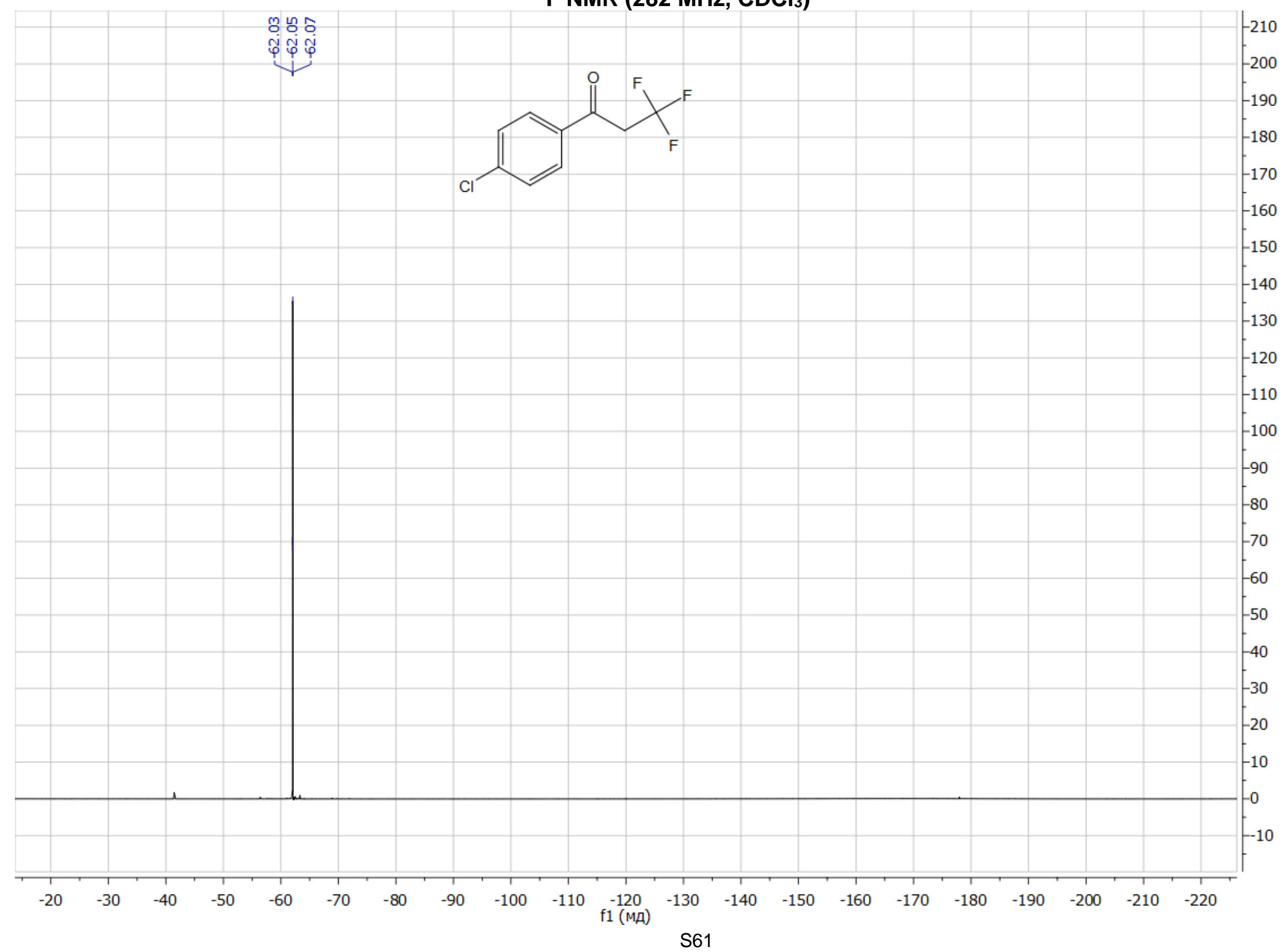


3,3,3-Trifluoro-1-(4-fluorophenyl)propan-1-one, $2 e$ ${ }^{1} \mathrm{H}$ NMR (300 MHz, $\mathrm{CDCl}_{3}$ )

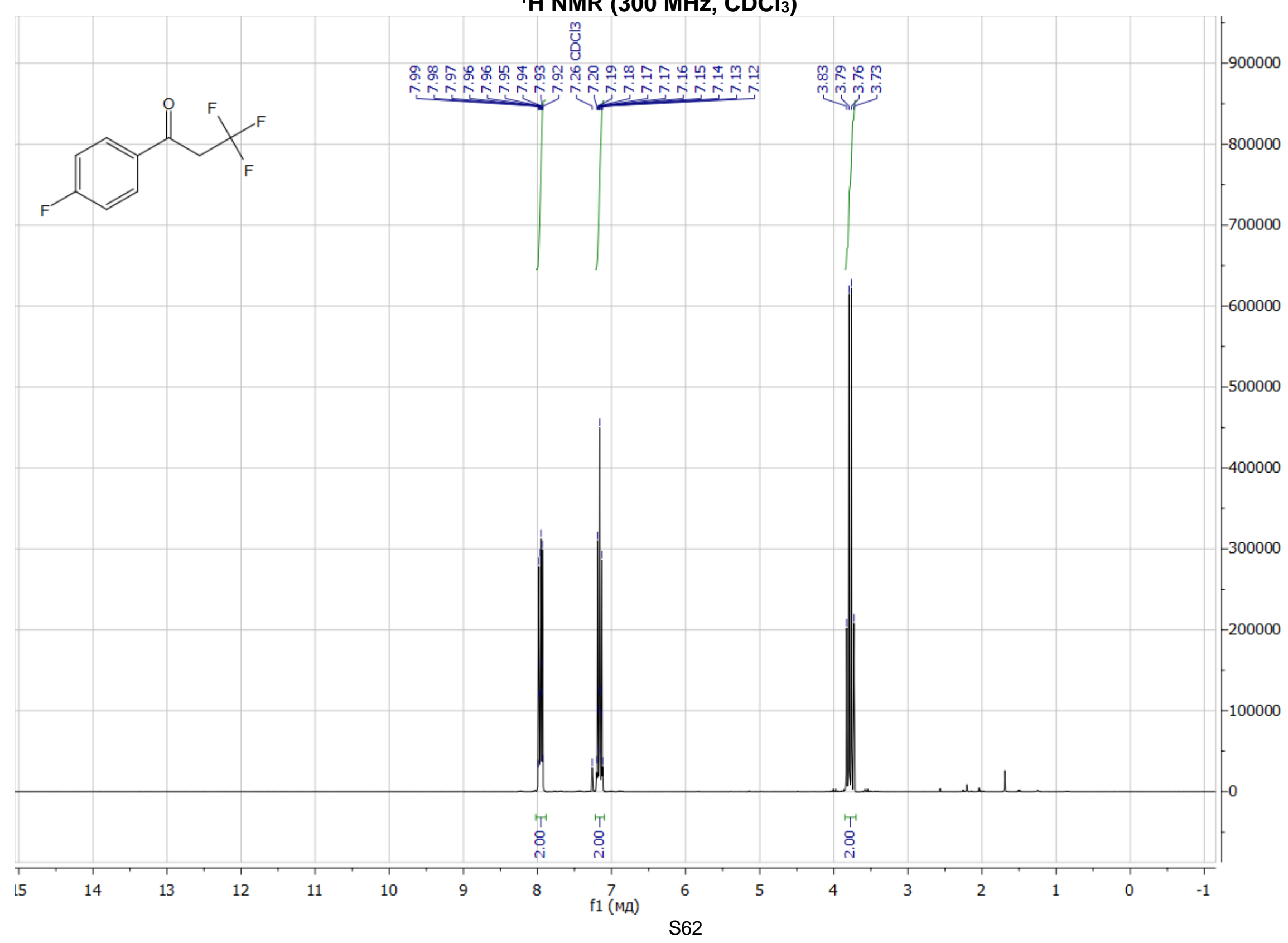


$\left.{ }^{13} \mathrm{C} \mathrm{NMR} \mathrm{(75} \mathrm{MHz,} \mathrm{CDCl}_{3}\right)$

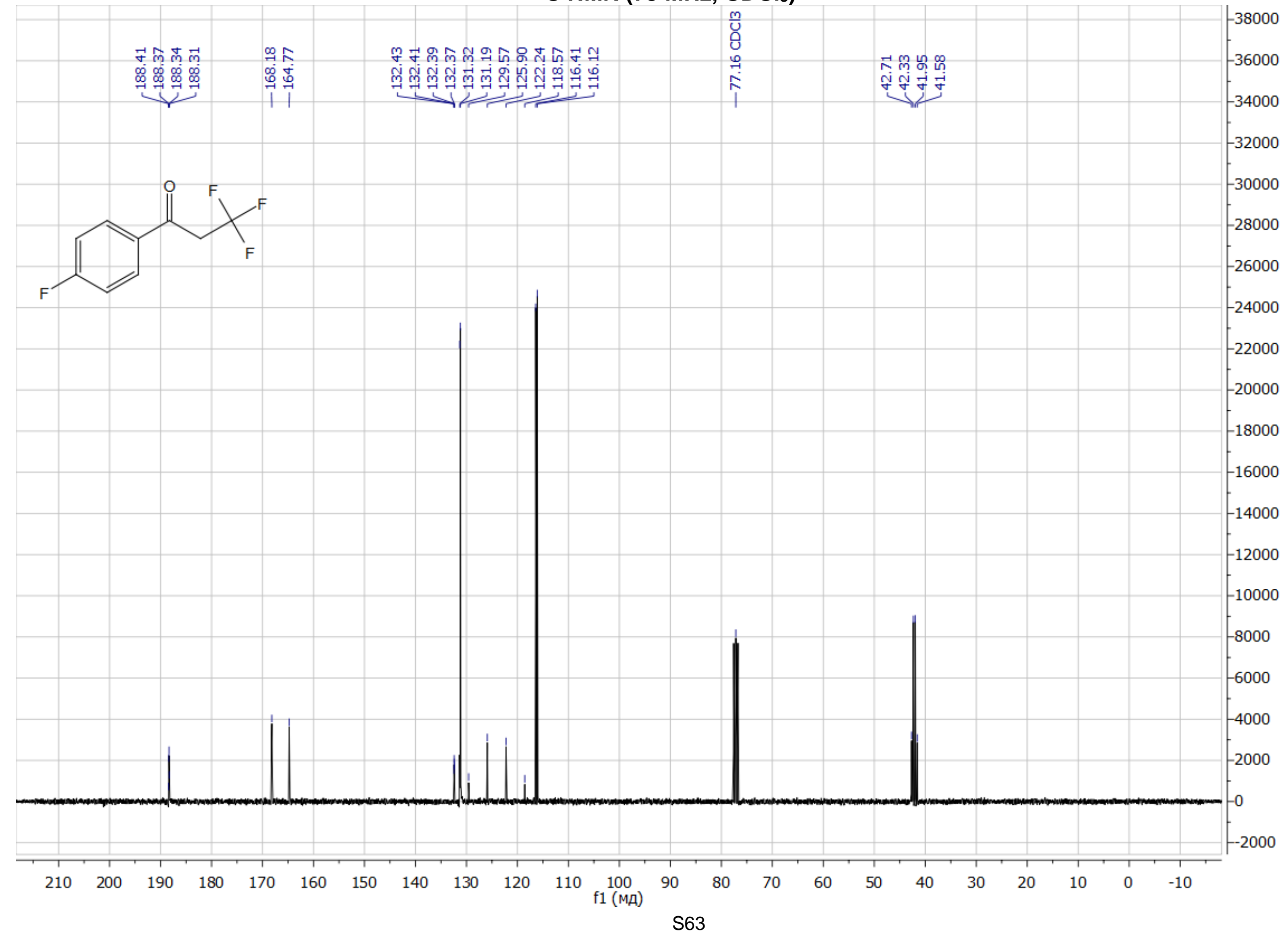


${ }^{19}$ F NMR (282 MHz, $\mathrm{CDCl}_{3}$ )

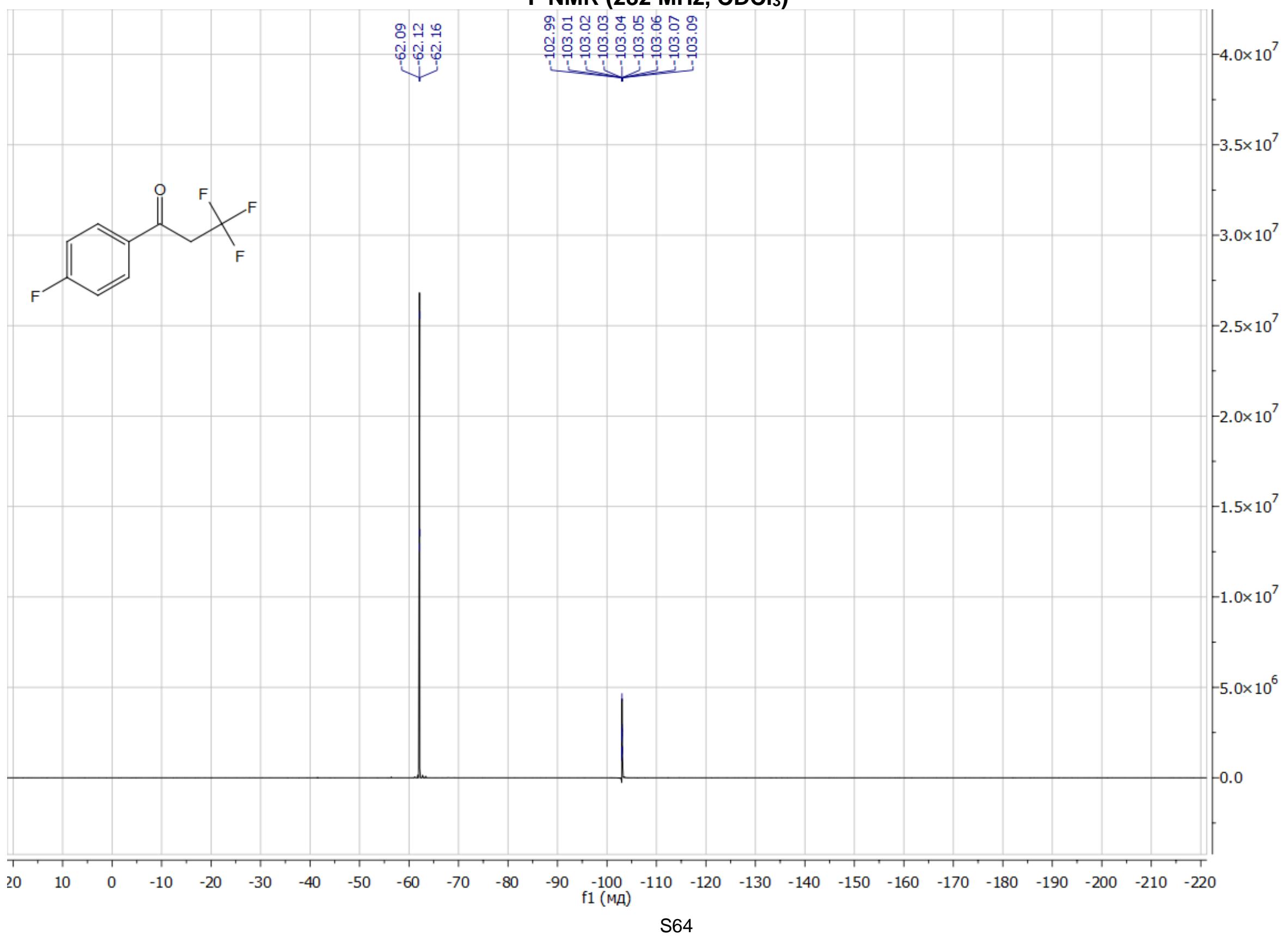


1-(4-Bromophenyl)-3,3,3-trifluoropropan-1-one, $2 f$

${ }^{1} \mathrm{H}$ NMR (300 MHz, CDCl 3 )

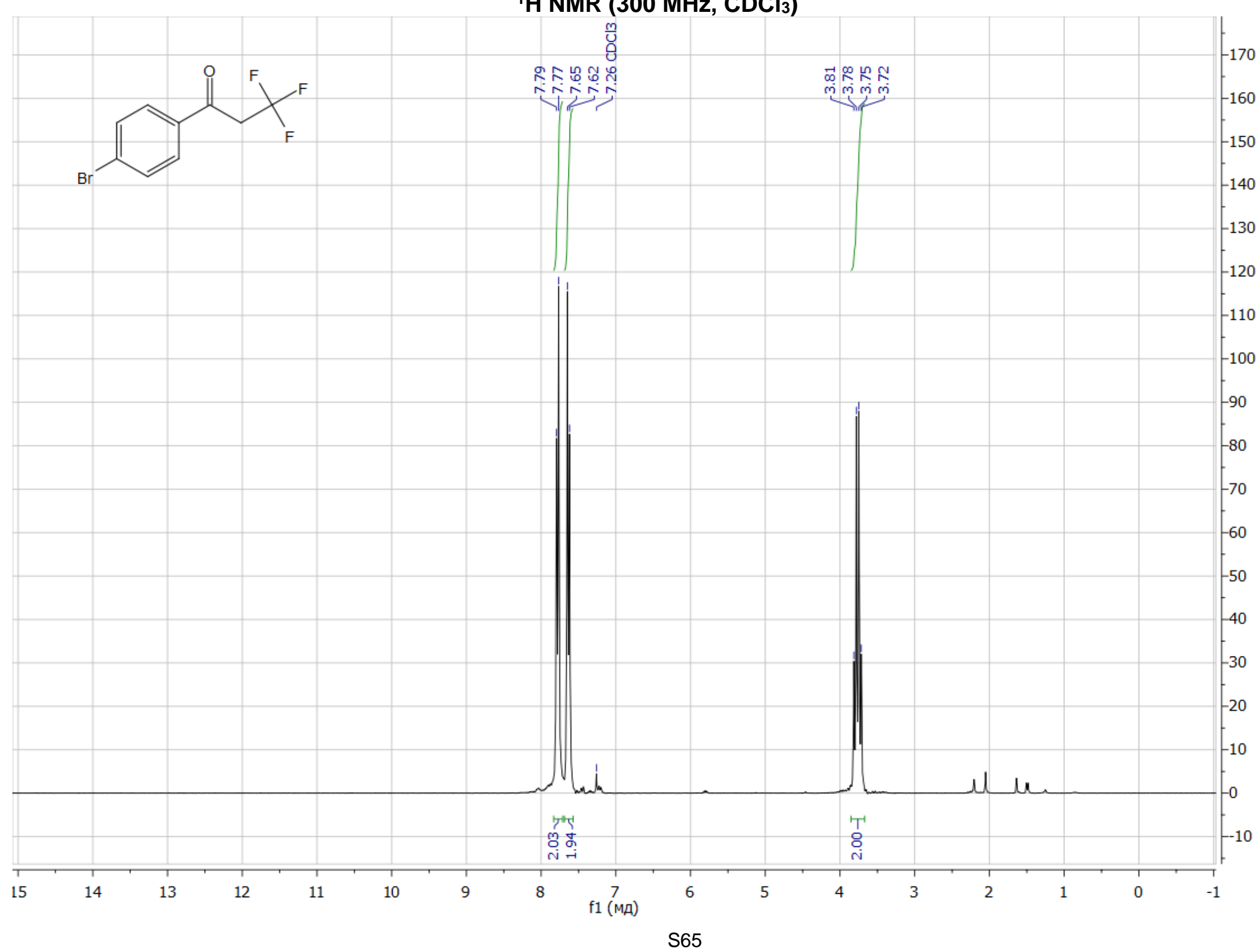


${ }^{13} \mathrm{C} \mathrm{NMR}\left(75 \mathrm{MHz}, \mathrm{CDCl}_{3}\right)$

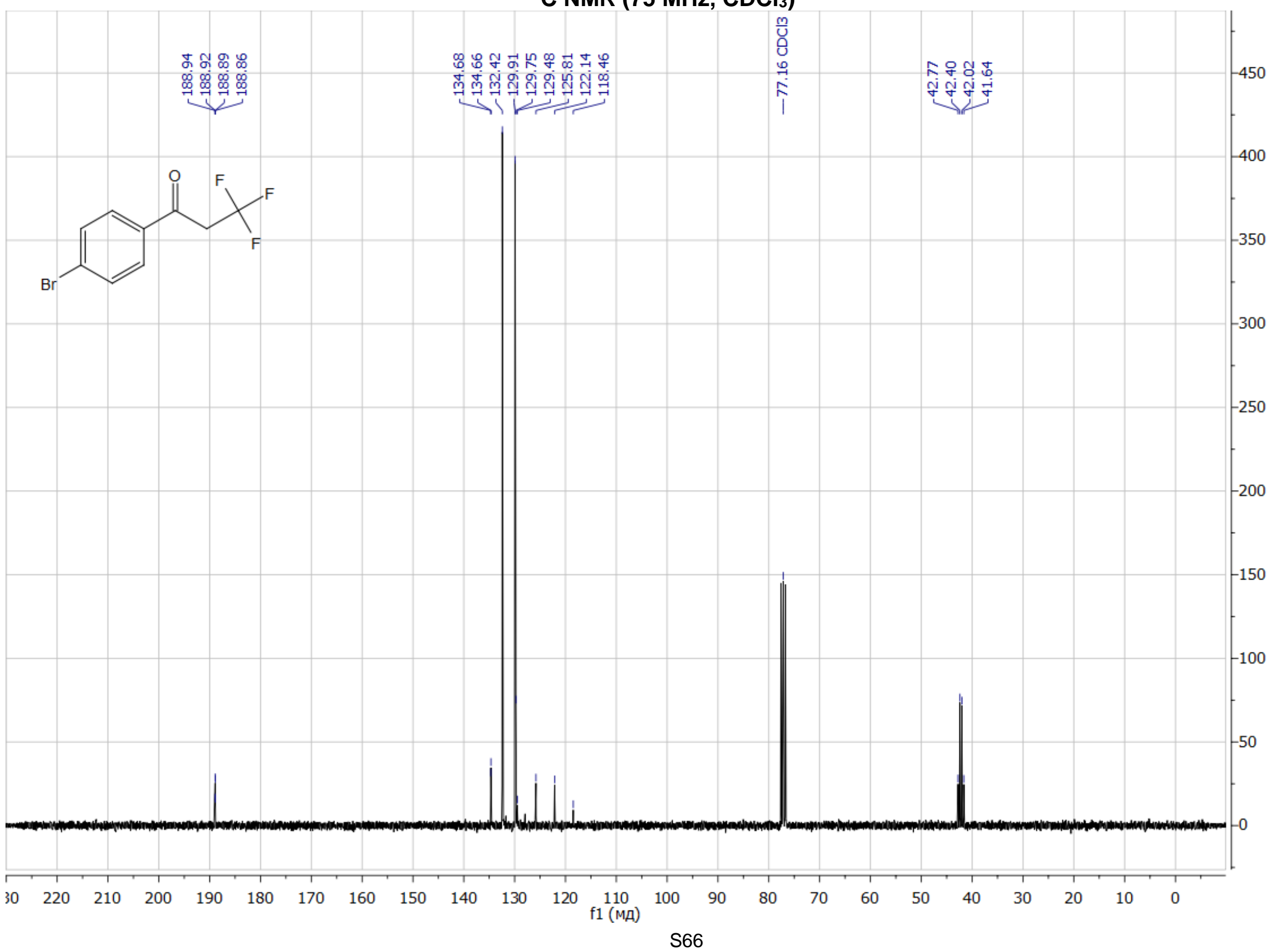


${ }^{19} \mathrm{~F}$ NMR (282 $\mathrm{MHz}, \mathrm{CDCl}_{3}$ )

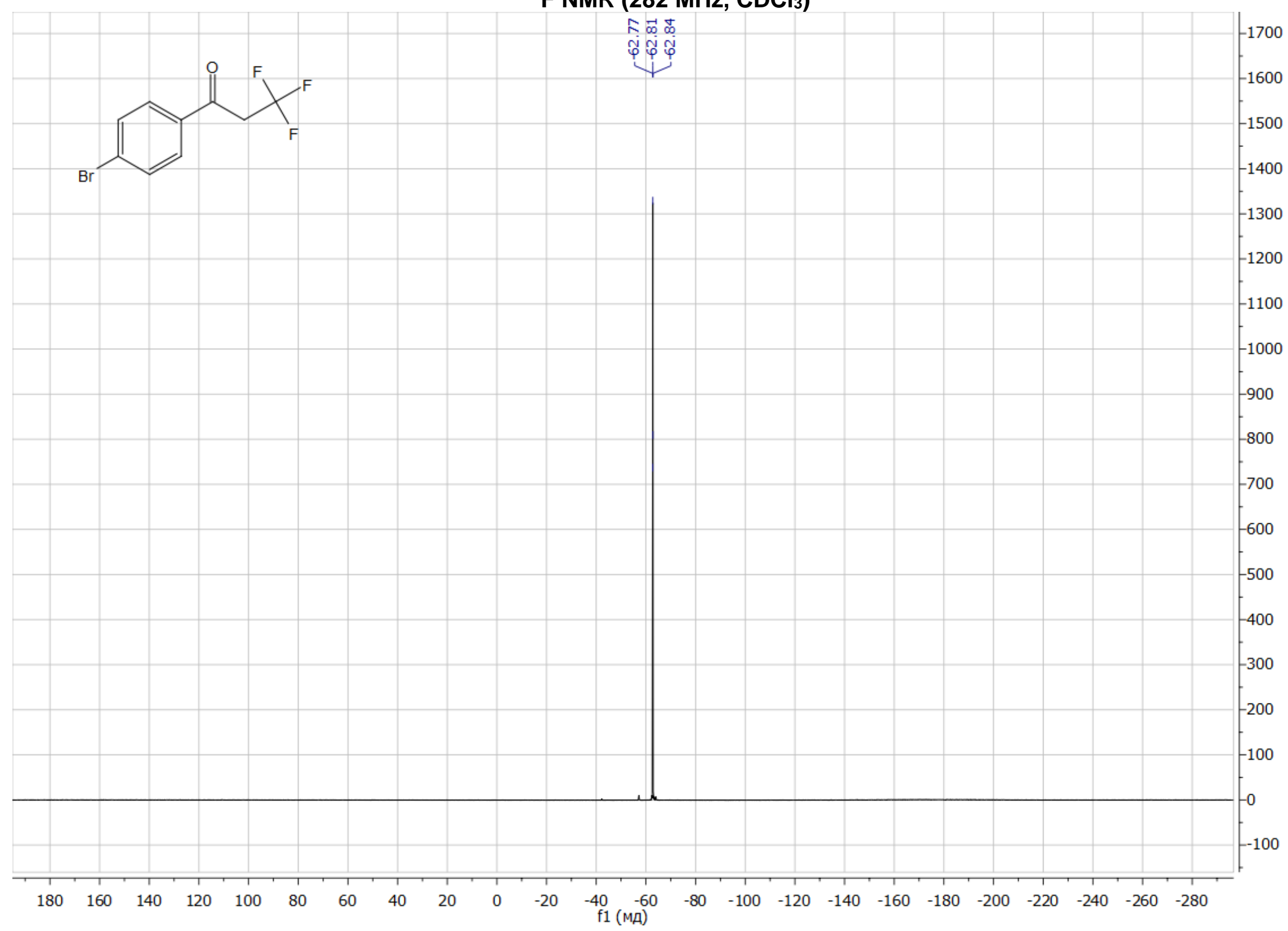


2-(Trifluoromethyl)-2,3-dihydro-1 $H$-inden-1-one, $2 \mathrm{~g}$

${ }^{1} \mathrm{H}$ NMR (300 MHz, CDCl 3 )

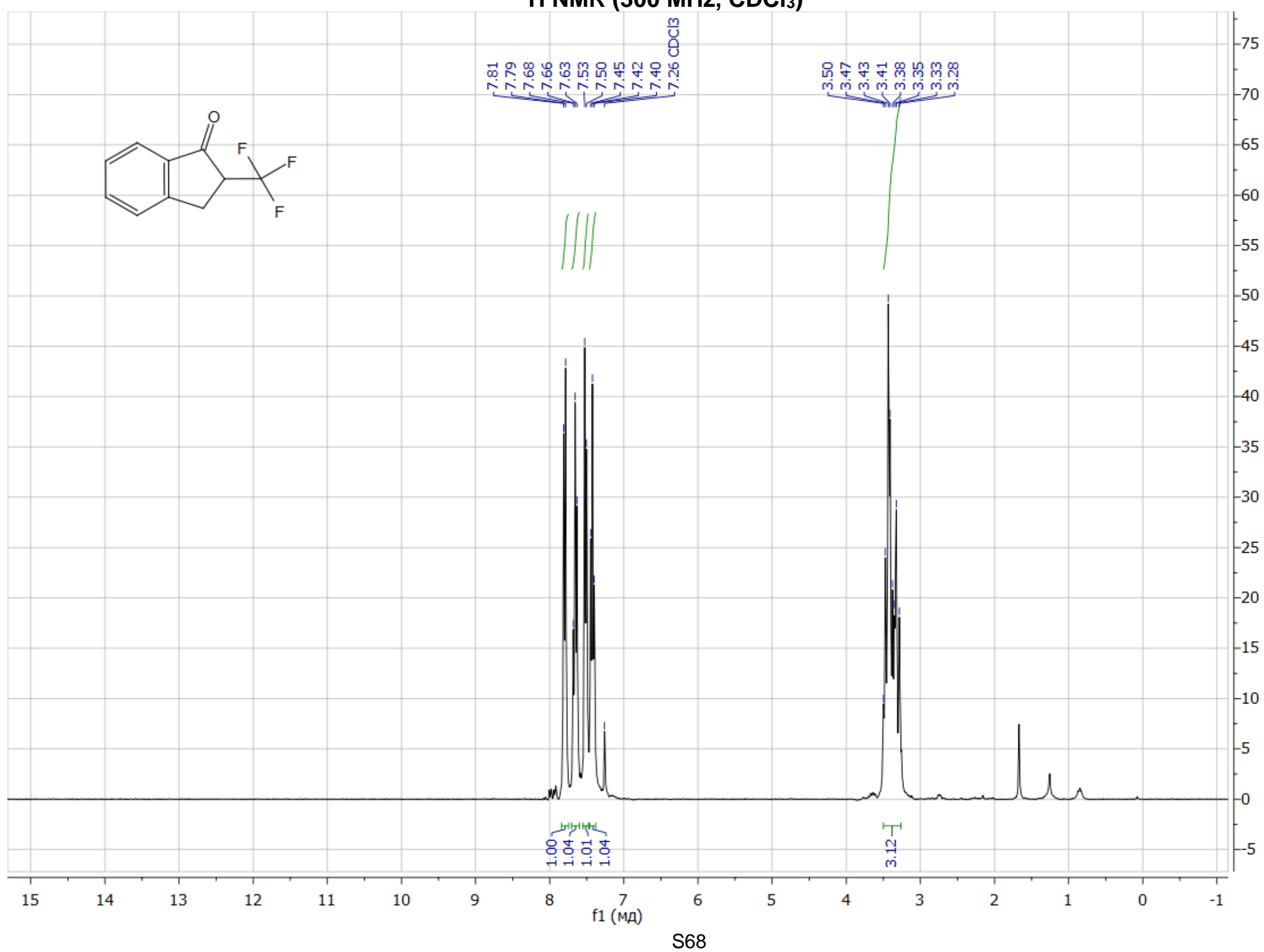


${ }^{13} \mathrm{C}$ NMR (75 MHz, $\left.\mathrm{CDCl}_{3}\right)$

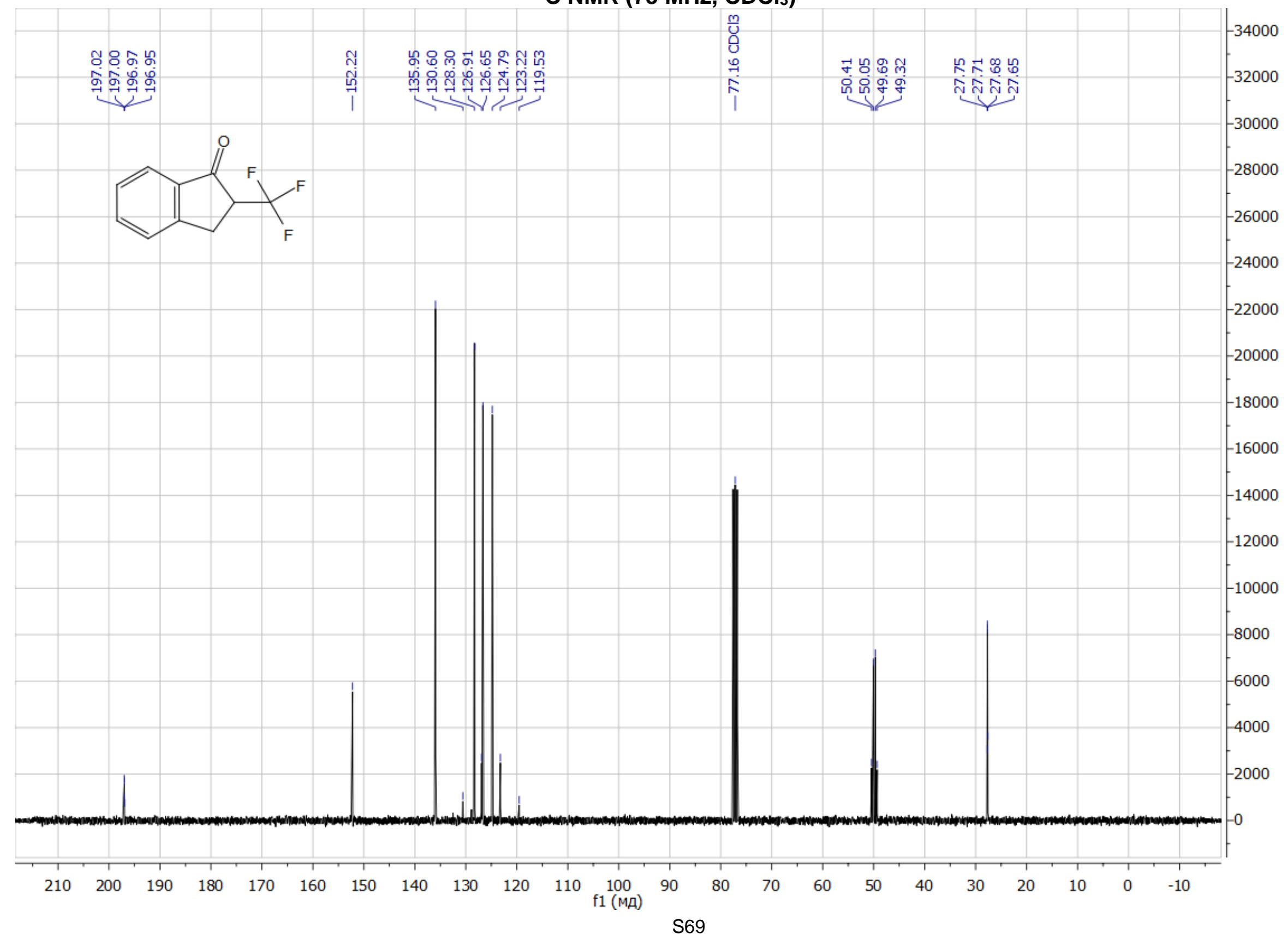


${ }^{19} \mathrm{~F}$ NMR (282 $\mathrm{MHz}, \mathrm{CDCl}_{3}$ )

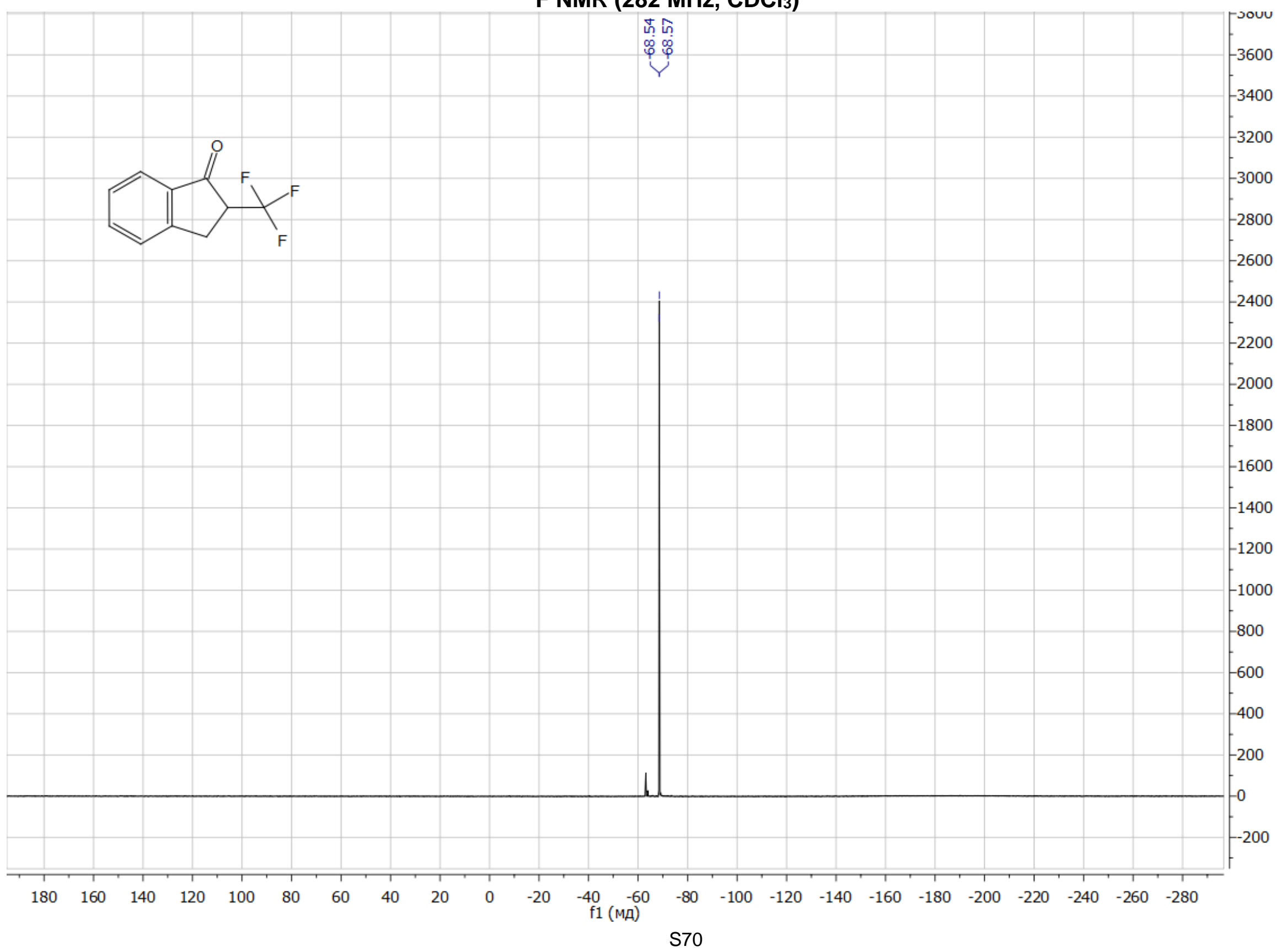


2-(Trifluoromethyl)-3,4-dihydronaphthalen-1(2H)-one, $2 \mathrm{~h}$

${ }^{1} \mathrm{H}$ NMR (300 MHz, CDCl 3 )

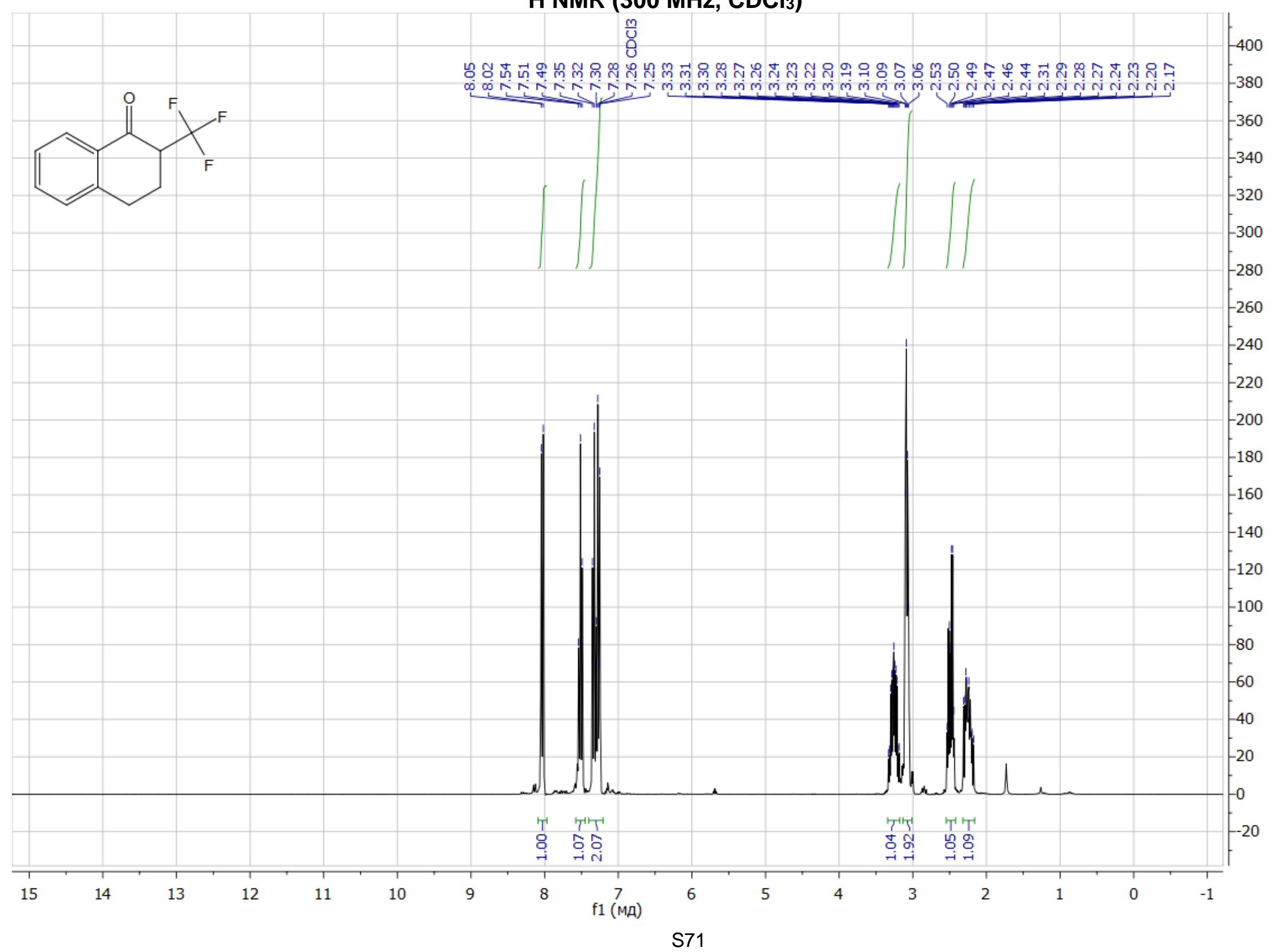


$\left.{ }^{13} \mathrm{C} \mathrm{NMR} \mathrm{(75} \mathrm{MHz,} \mathrm{CDCl}_{3}\right)$

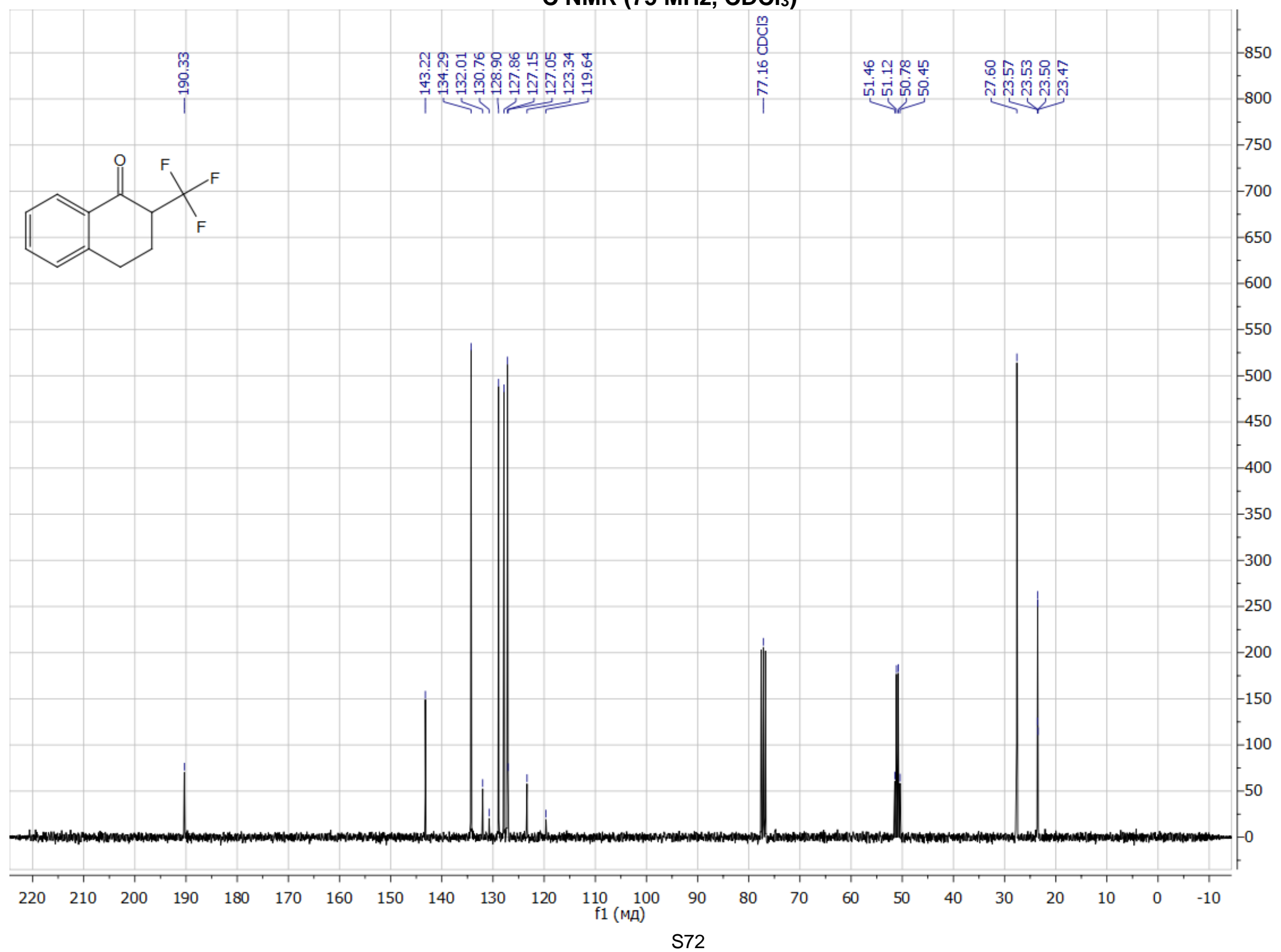


${ }^{19} \mathrm{~F}$ NMR (282 $\left.\mathrm{MHz}, \mathrm{CDCl}_{3}\right)$

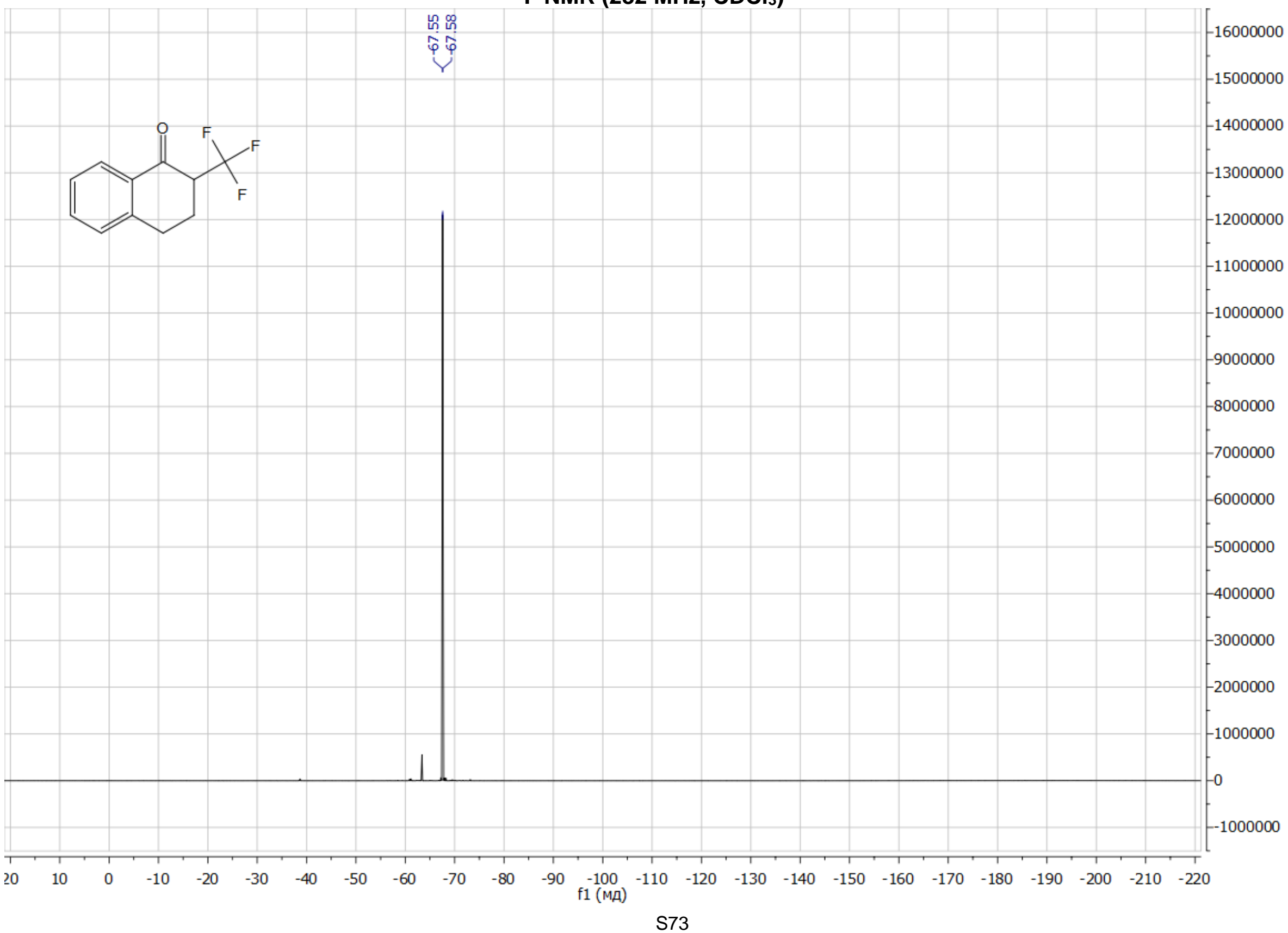


3,3,3-Trifluoro-2-methyl-1-phenylpropan-1-one, $2 \mathrm{i}$

${ }^{1} \mathrm{H}$ NMR (300 MHz, $\mathrm{CDCl}_{3}$ )

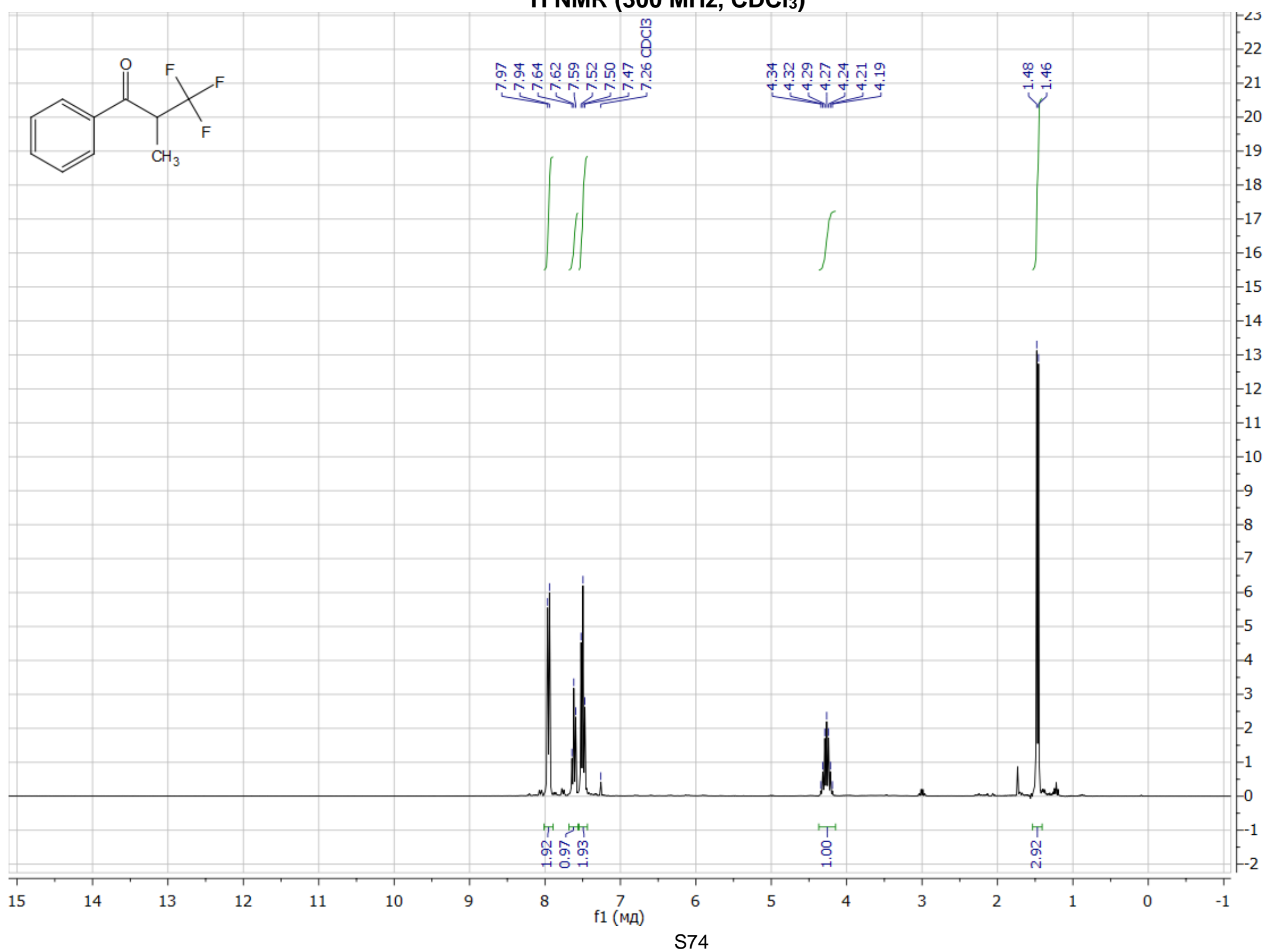


${ }^{13} \mathrm{C}$ NMR (75 MHz, $\left.\mathrm{CDCl}_{3}\right)$

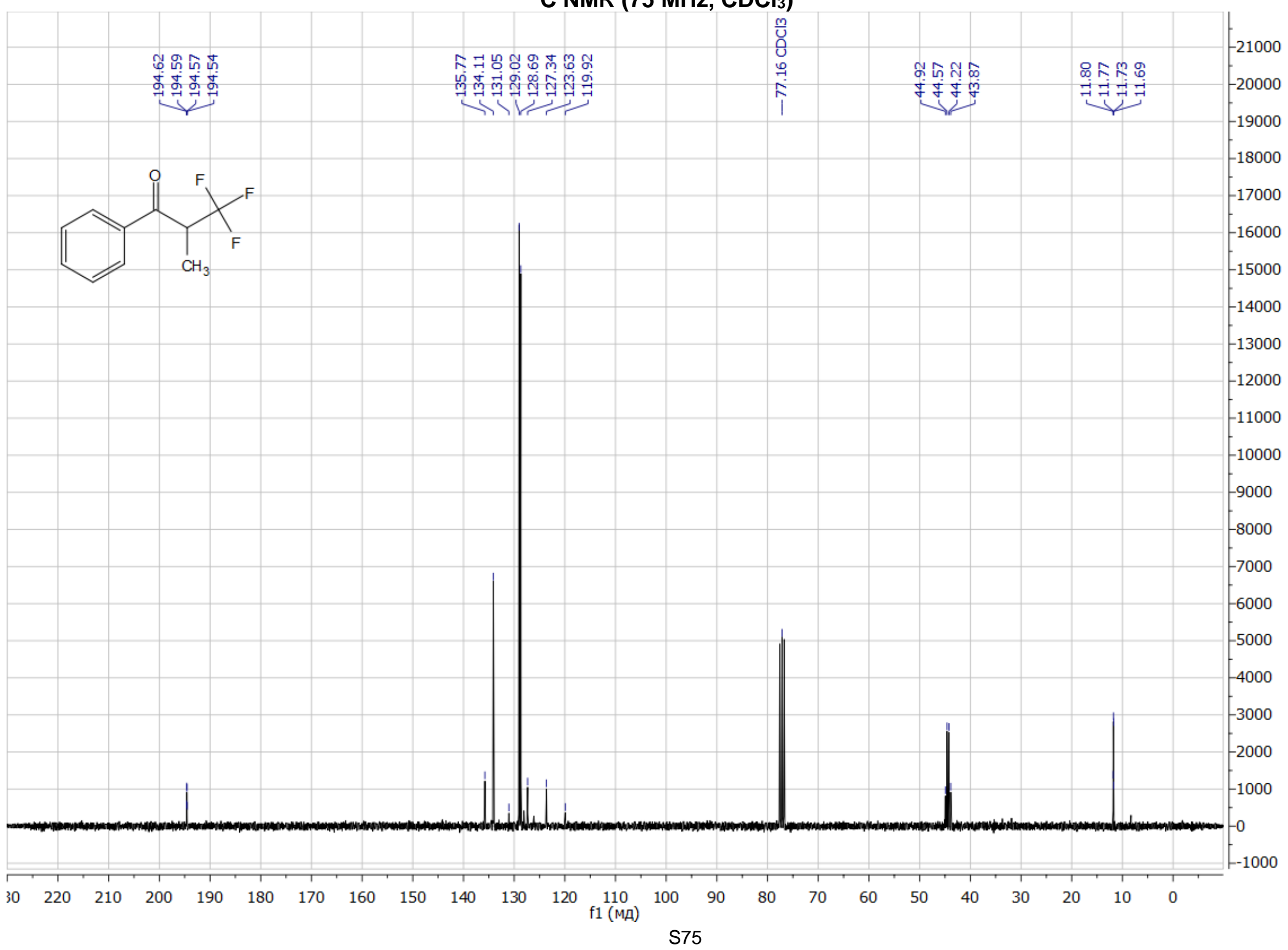


${ }^{19} \mathrm{~F}$ NMR (282 $\mathrm{MHz}, \mathrm{CDCl}_{3}$ )

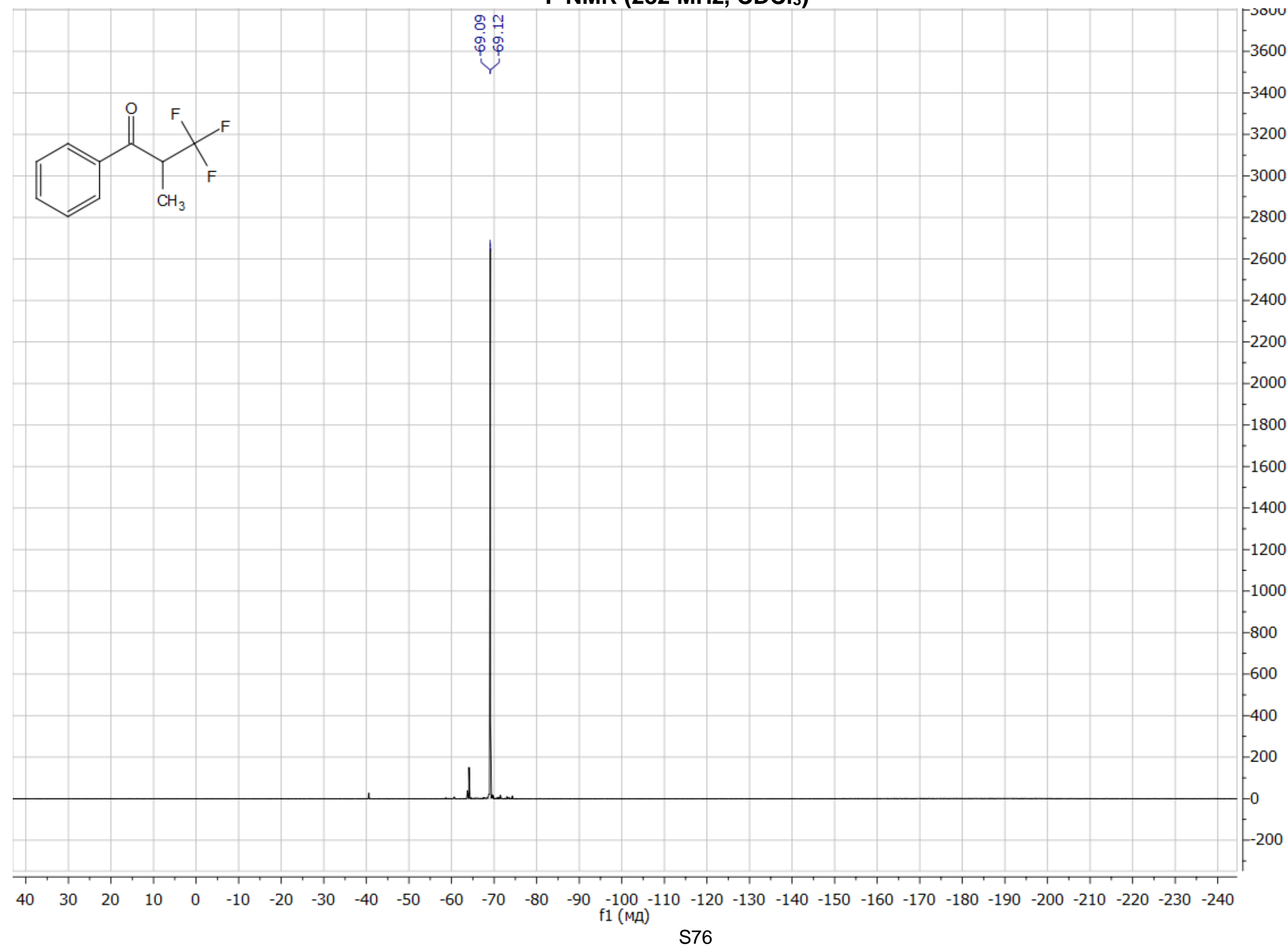


3,3,3-Trifluoro-1-(naphthalen-2-yl)propan-1-one, 2j

${ }^{1} \mathrm{H}$ NMR $\left(300 \mathrm{MHz}, \mathrm{CDCl}_{3}\right)$

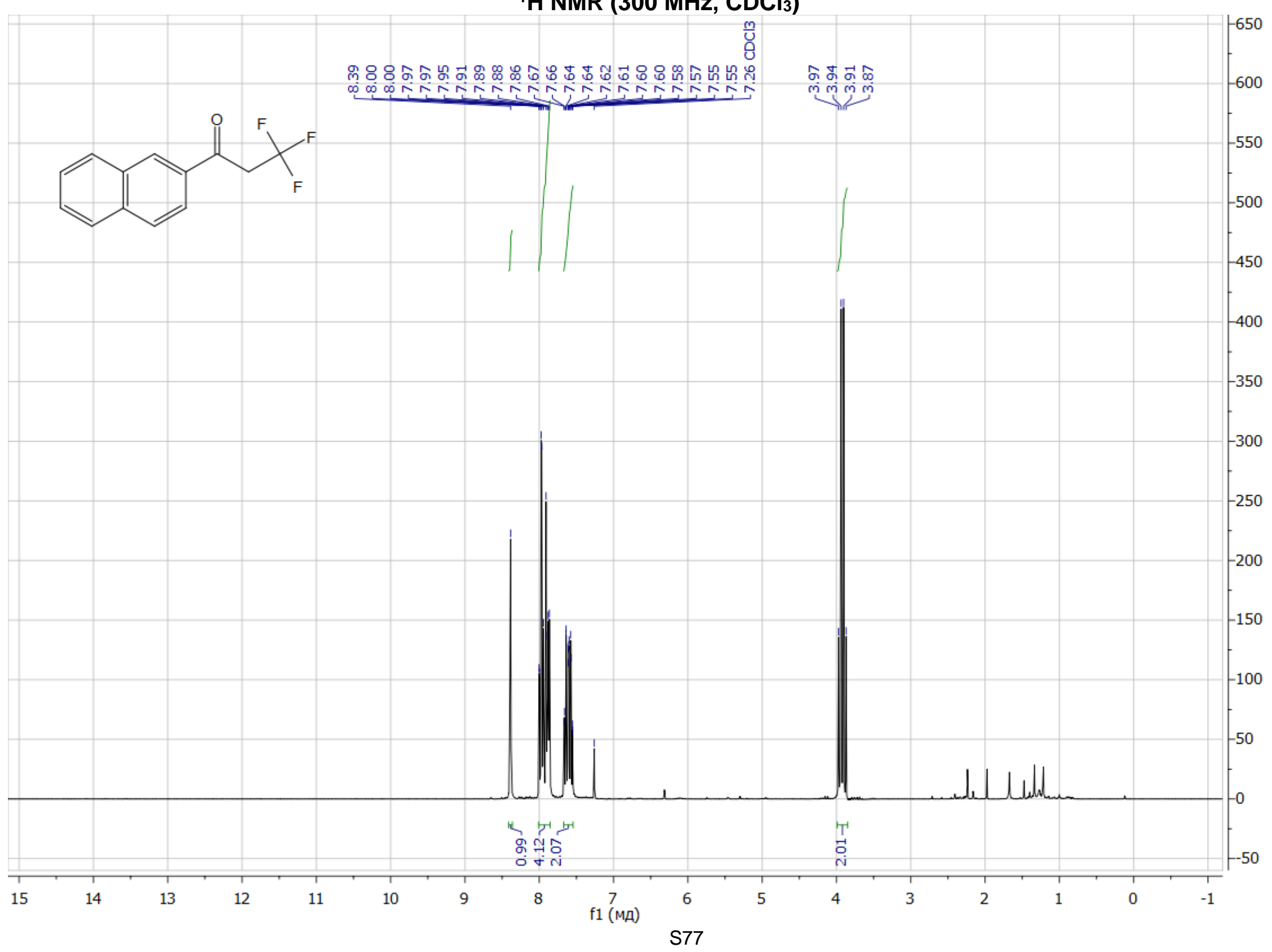


$\left.{ }^{13} \mathrm{C} \mathrm{NMR} \mathrm{(75} \mathrm{MHz,} \mathrm{CDCl}_{3}\right)$

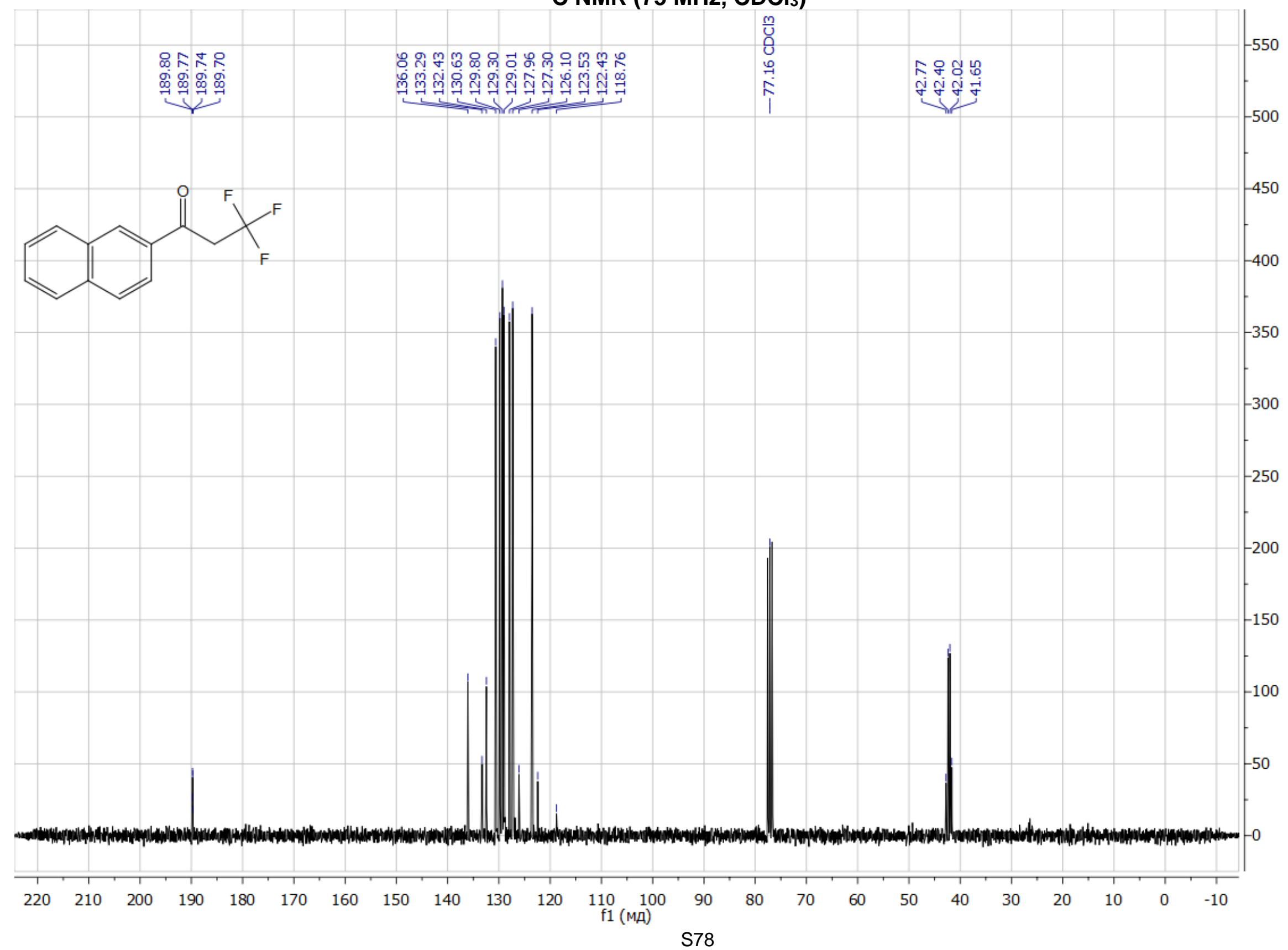


${ }^{19} \mathrm{~F}$ NMR (282 MHz, $\left.\mathrm{CDCl}_{3}\right)$

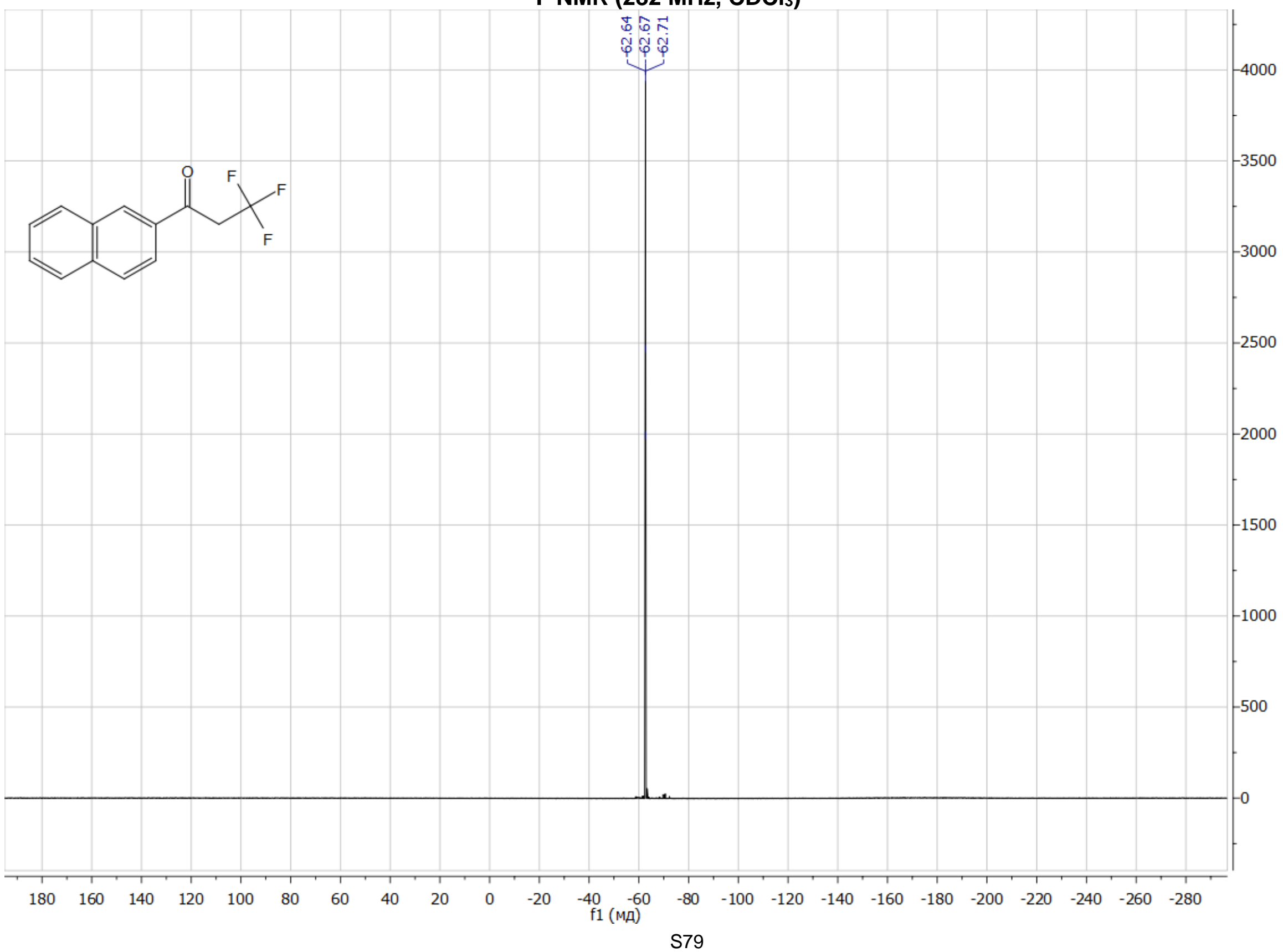


2-(Trifluoromethyl)cyclopentan-1-one, 2k

${ }^{1} \mathrm{H}$ NMR (300 MHz, $\mathrm{CDCl}_{3}$ )

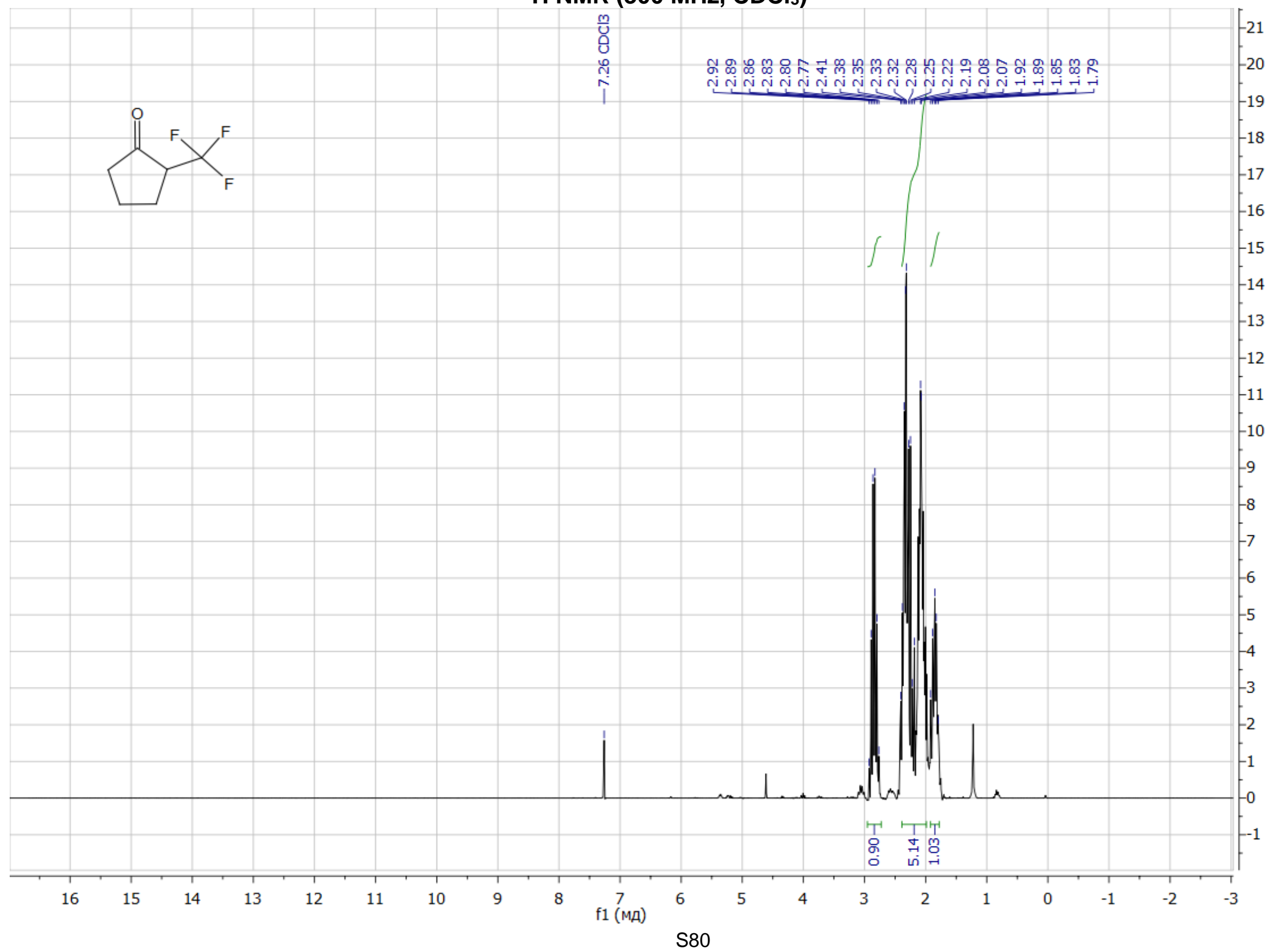


$\left.{ }^{13} \mathrm{C} \mathrm{NMR} \mathrm{(75} \mathrm{MHz,} \mathrm{CDCl}_{3}\right)$

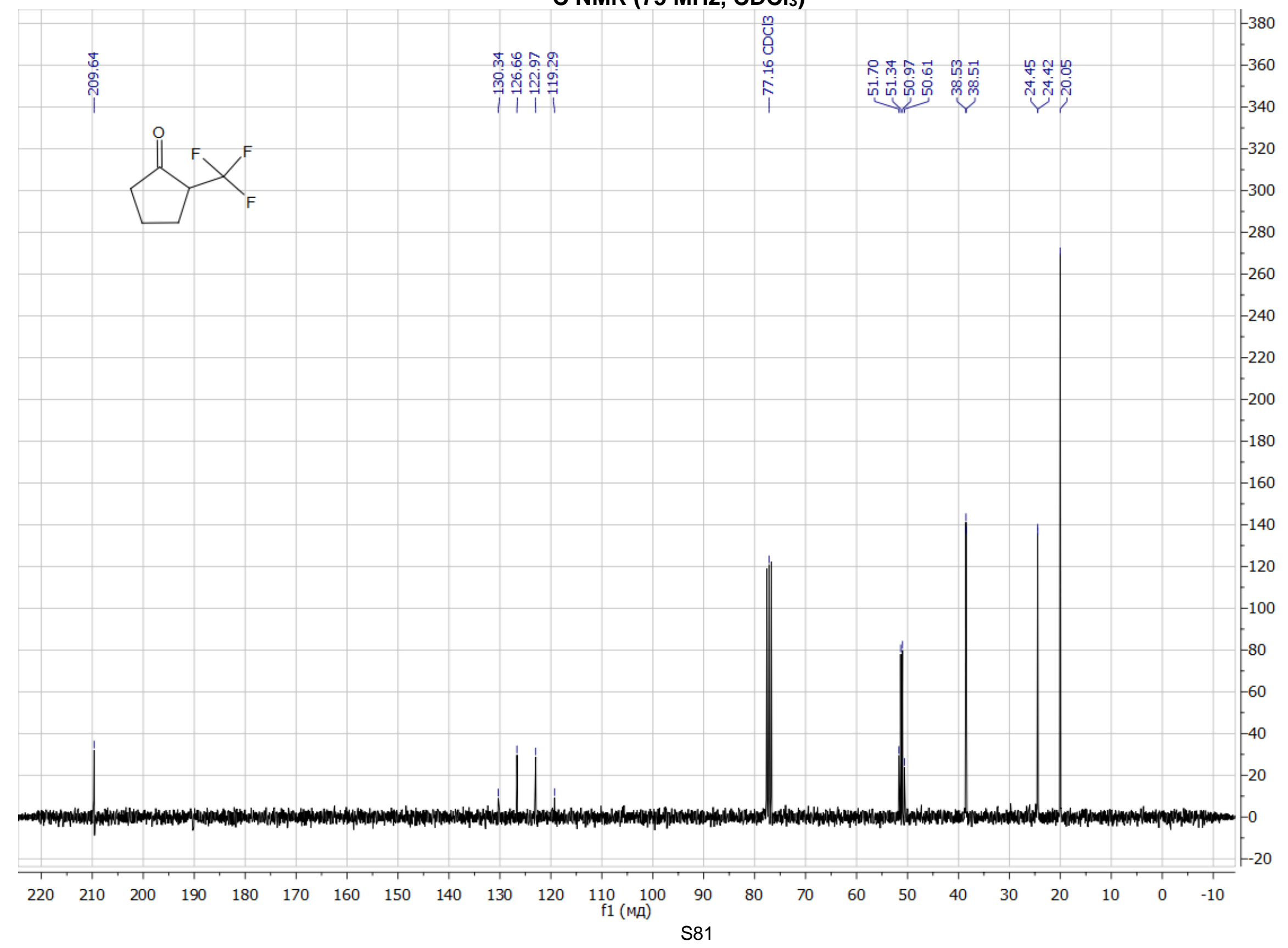


${ }^{19} \mathrm{~F}$ NMR (282 $\mathrm{MHz}, \mathrm{CDCl}_{3}$ )

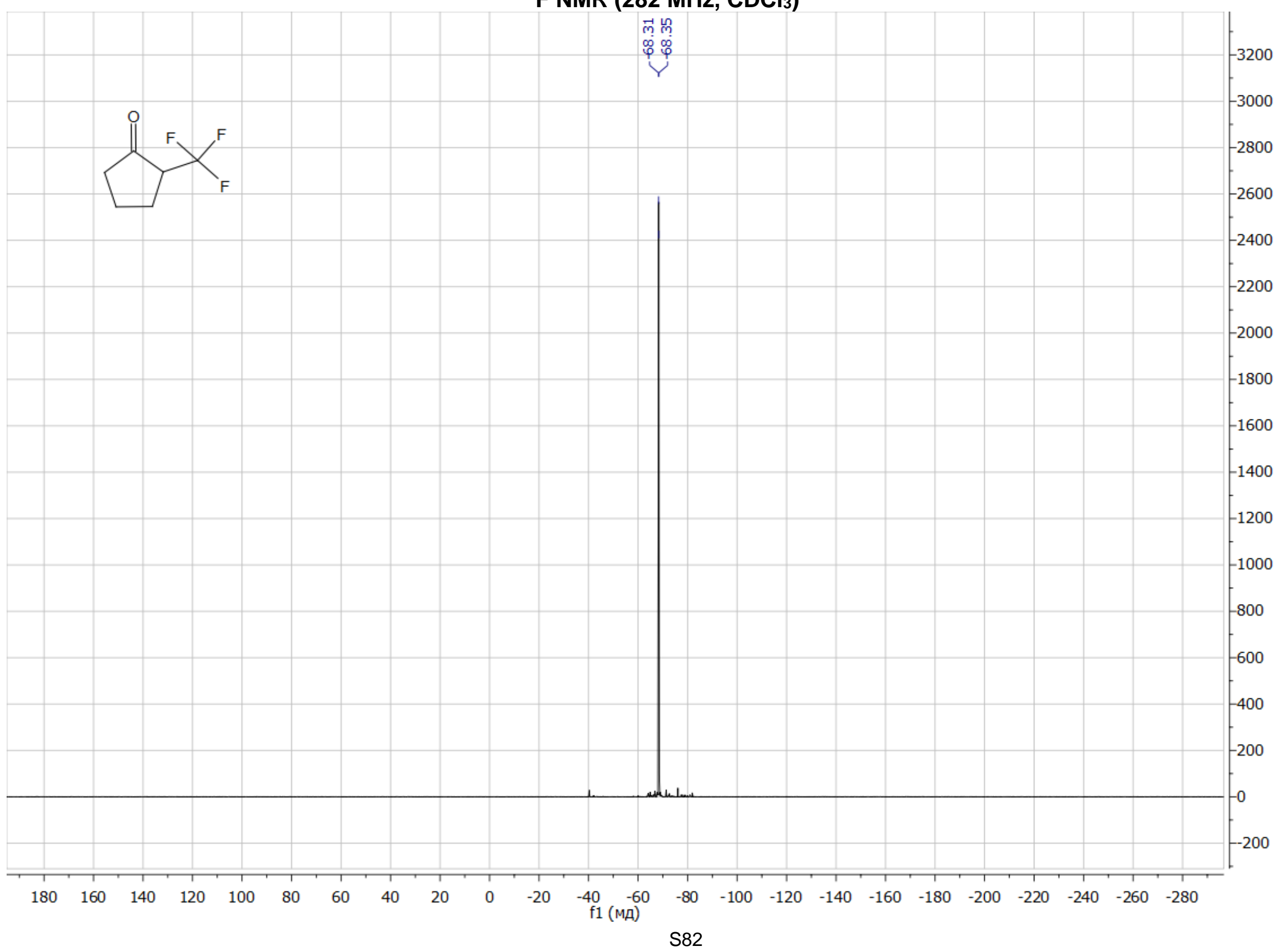


2-(Trifluoromethyl)cyclohexan-1-one, 2I

${ }^{1} \mathrm{H}$ NMR (300 MHz, $\mathrm{CDCl}_{3}$ )

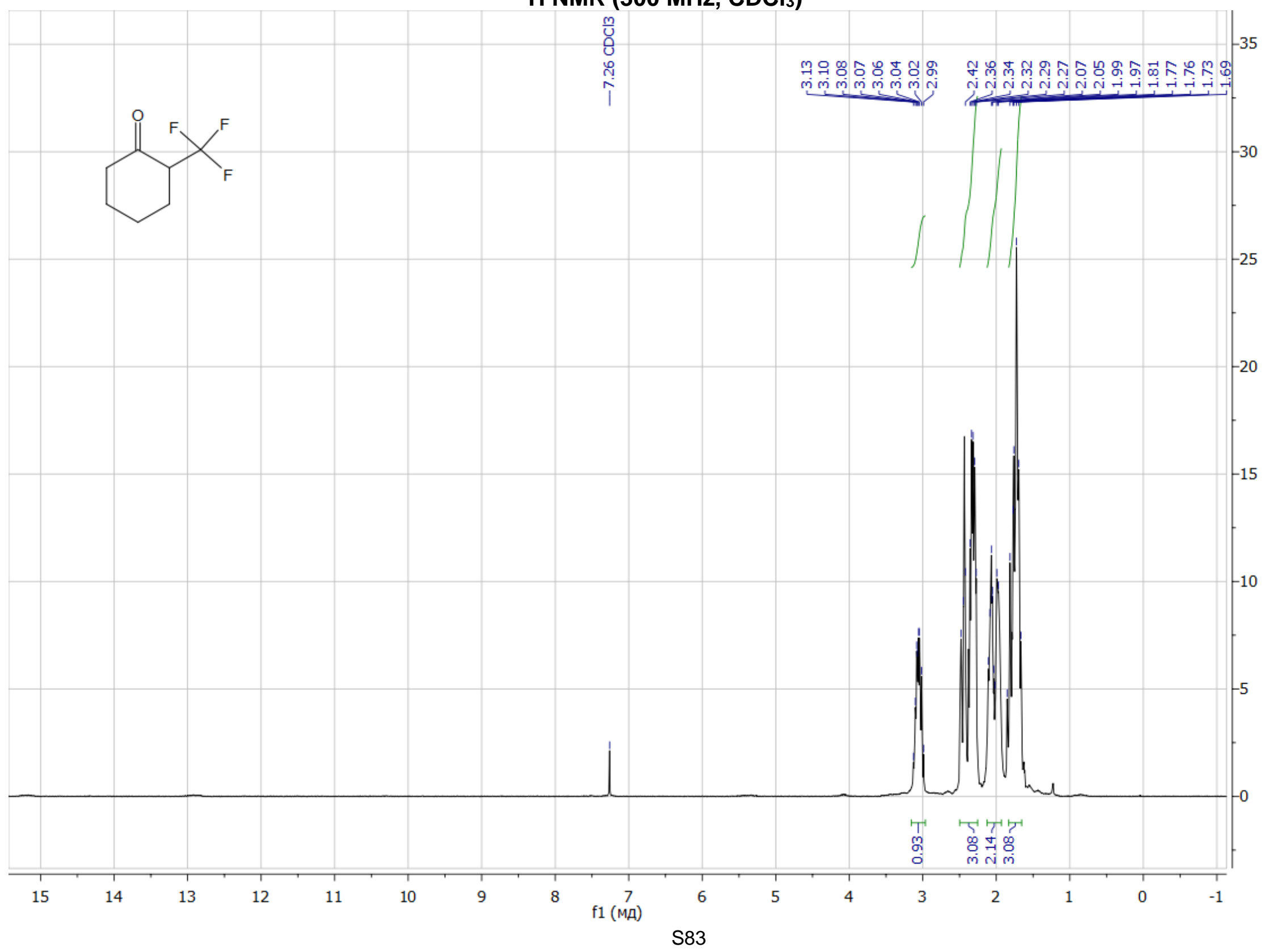


${ }^{13} \mathrm{C}$ NMR (75 MHz, $\left.\mathrm{CDCl}_{3}\right)$

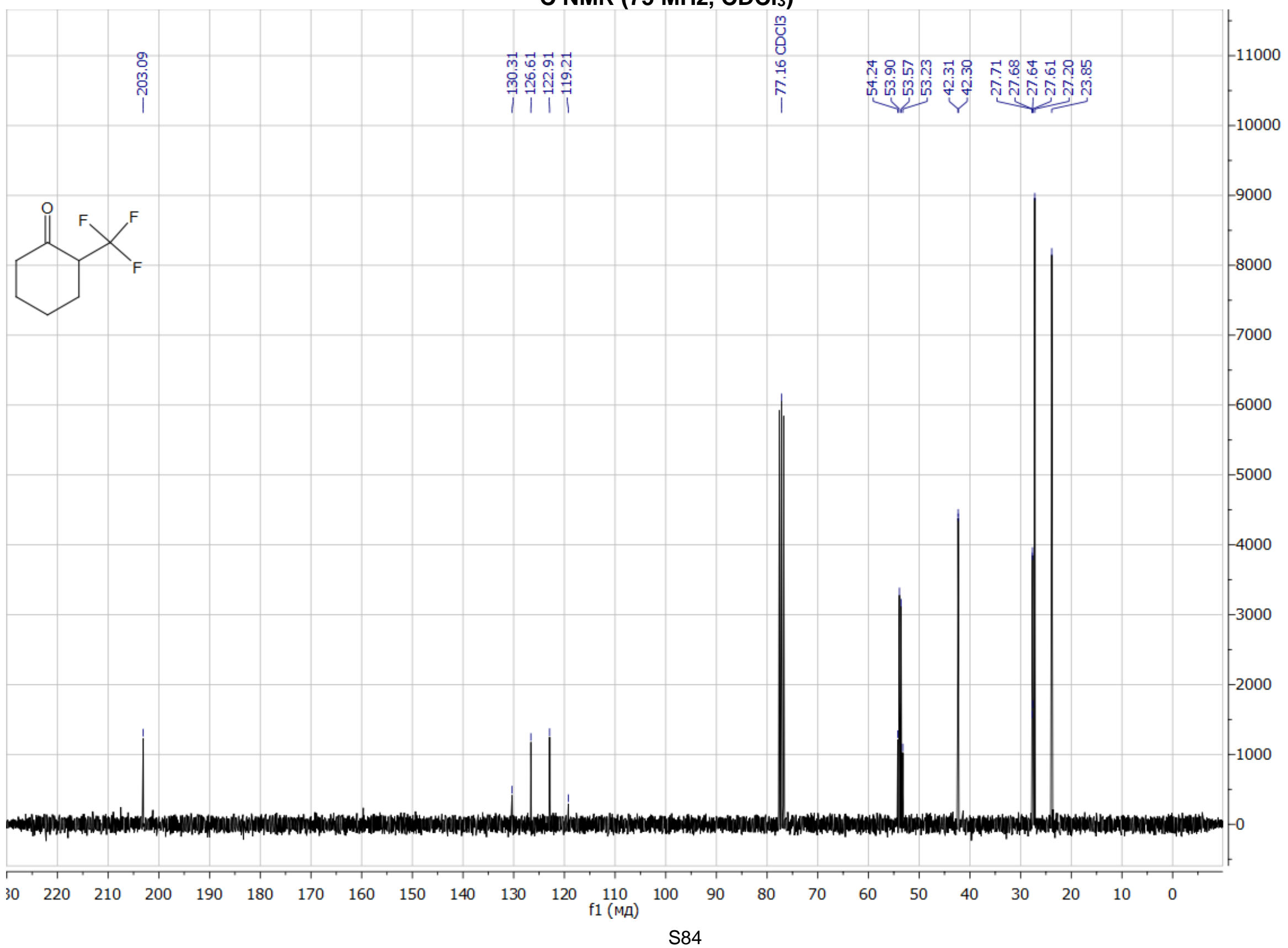


${ }^{19} \mathrm{~F}$ NMR (282 MHz, $\left.\mathrm{CDCl}_{3}\right)$

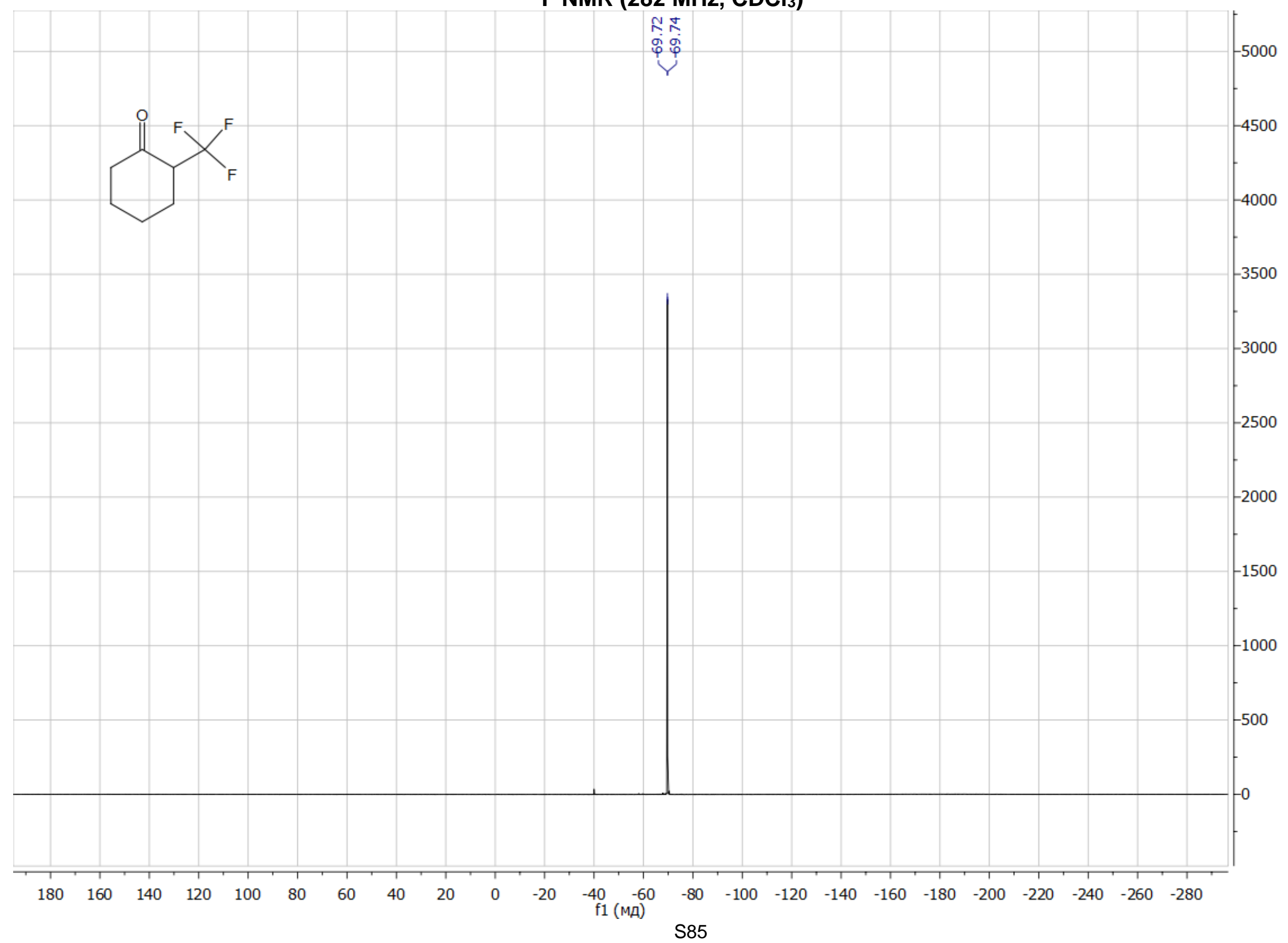


4-(Tert-butyl)-2-(trifluoromethyl)cyclohexan-1-one, $2 \mathrm{~m}$

${ }^{1} \mathrm{H}$ NMR (300 MHz, CDCl 3 )

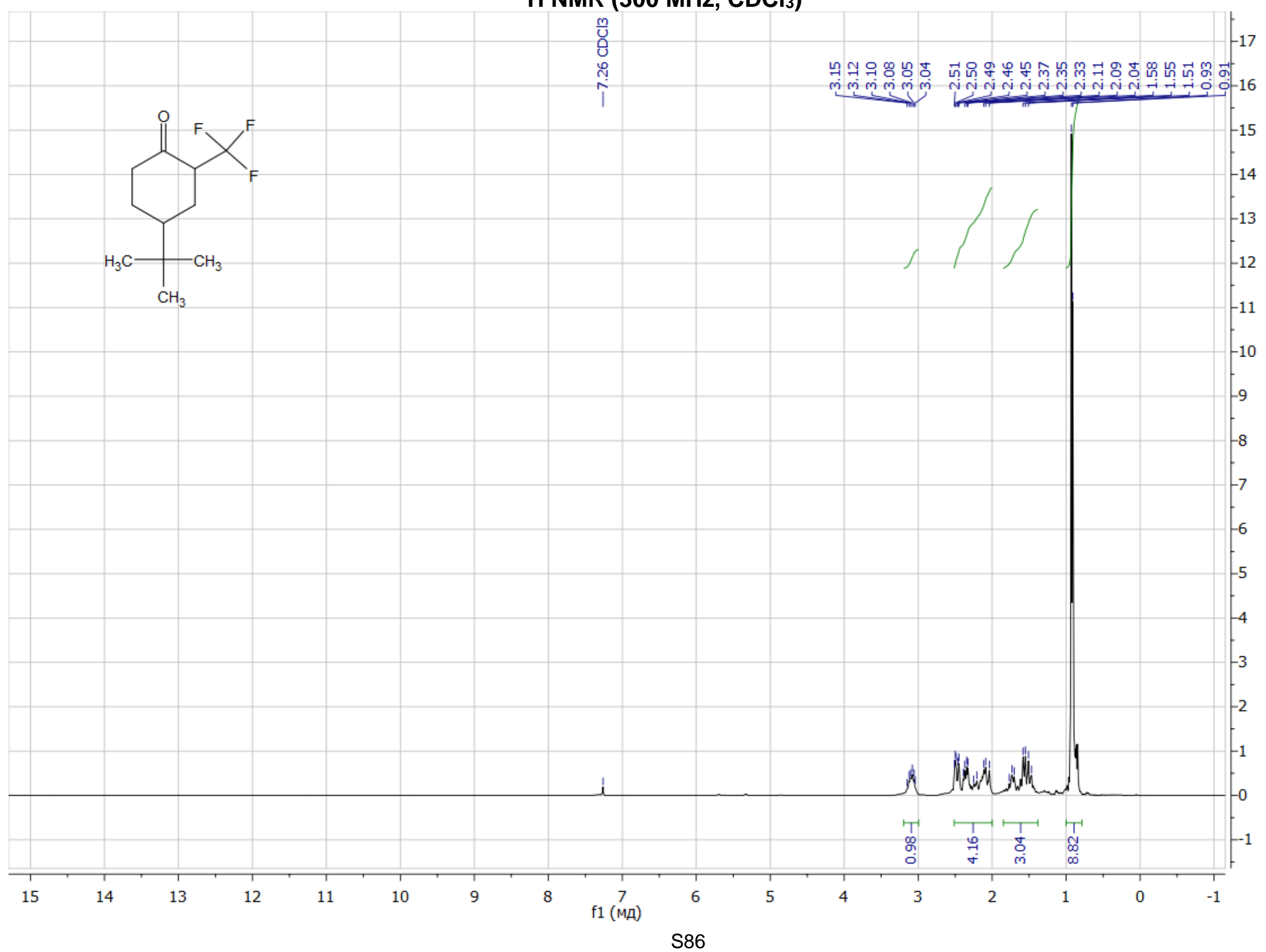


${ }^{13} \mathrm{C} \mathrm{NMR}\left(75 \mathrm{MHz}, \mathrm{CDCl}_{3}\right)$

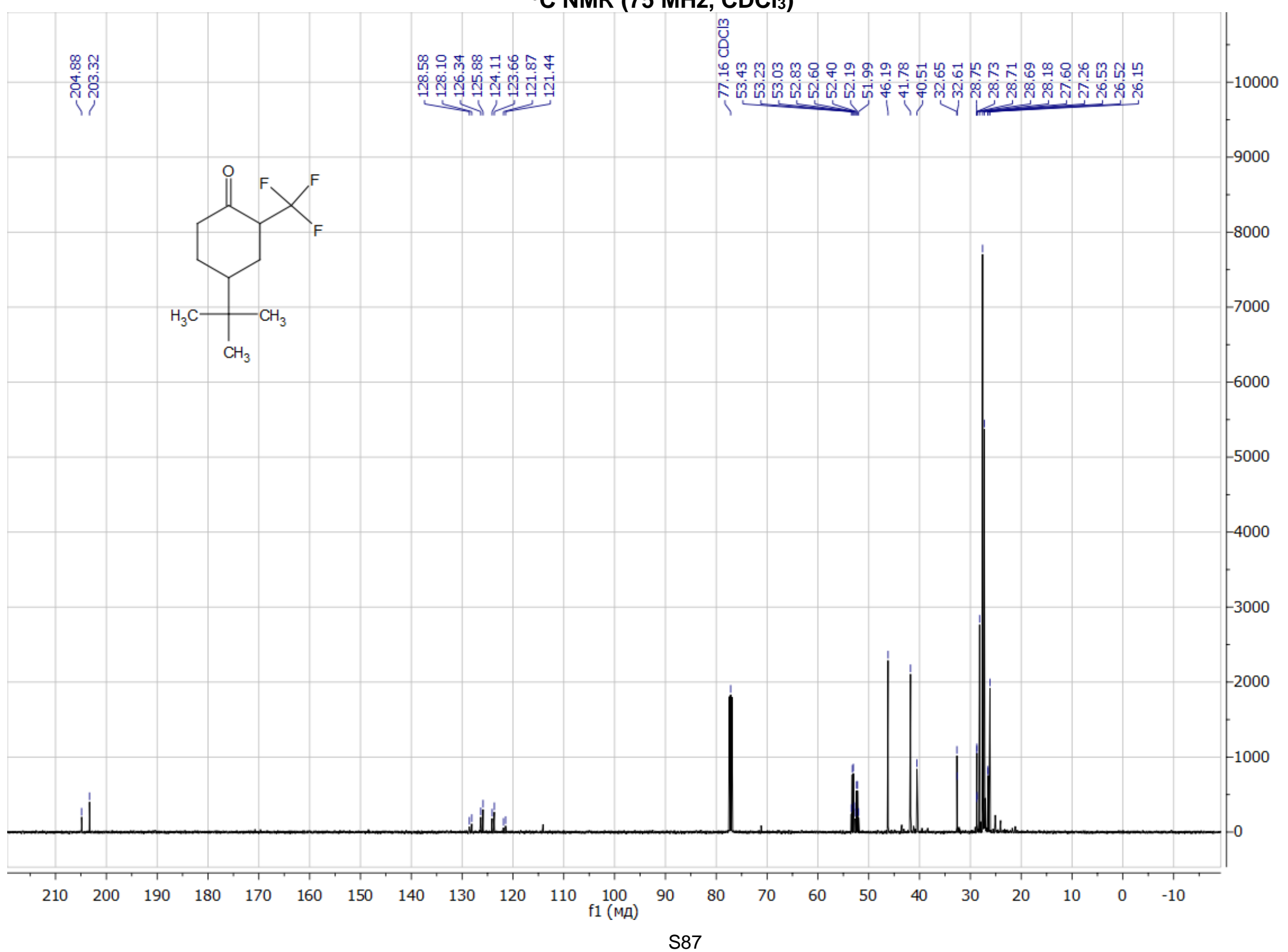


${ }^{19} \mathrm{~F}$ NMR (282 MHz, $\left.\mathrm{CDCl}_{3}\right)$

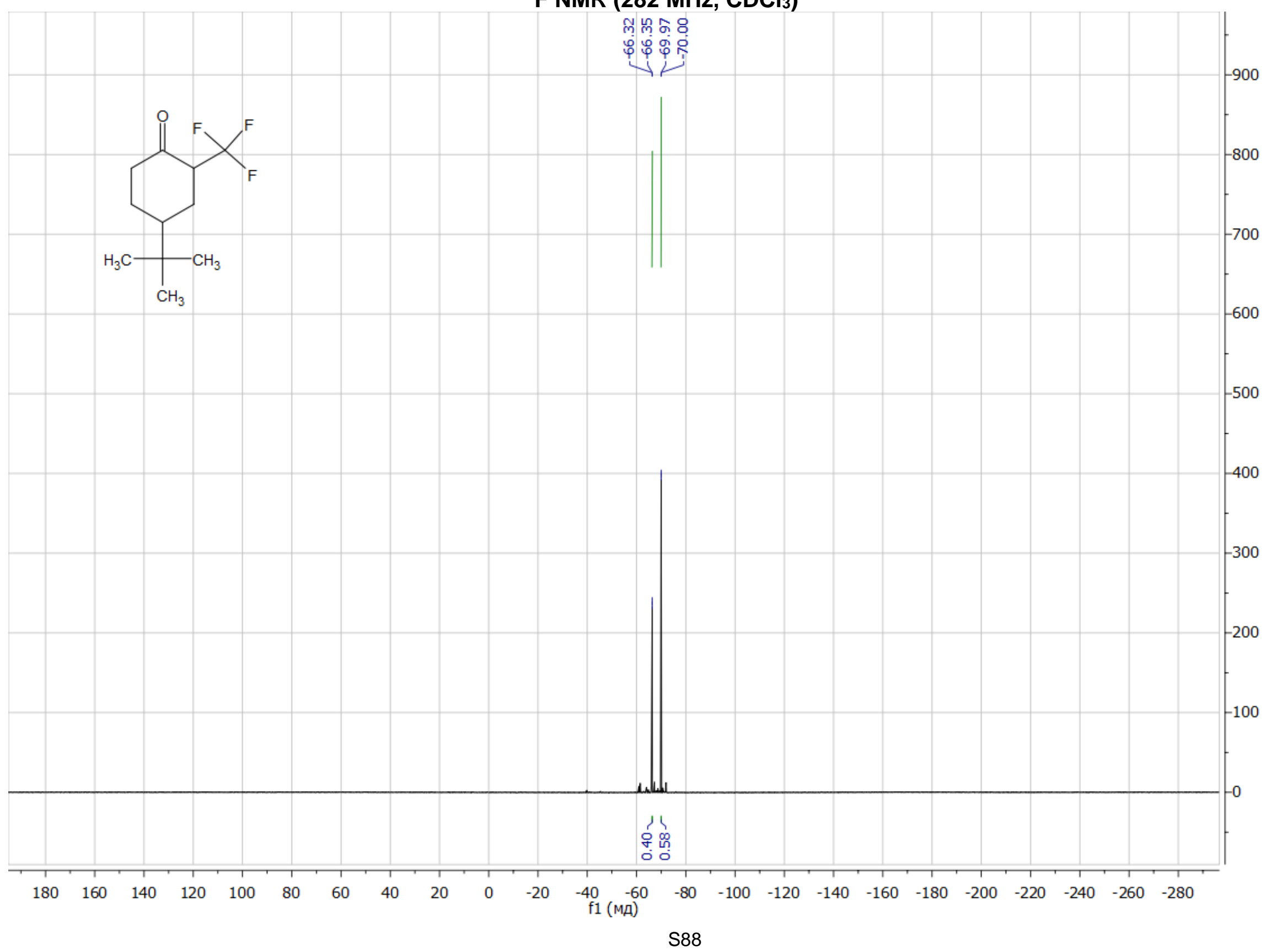


2-(Trifluoromethyl)cycloheptan-1-one, 2n

${ }^{1} \mathrm{H}$ NMR (300 MHz, $\mathrm{CDCl}_{3}$ )

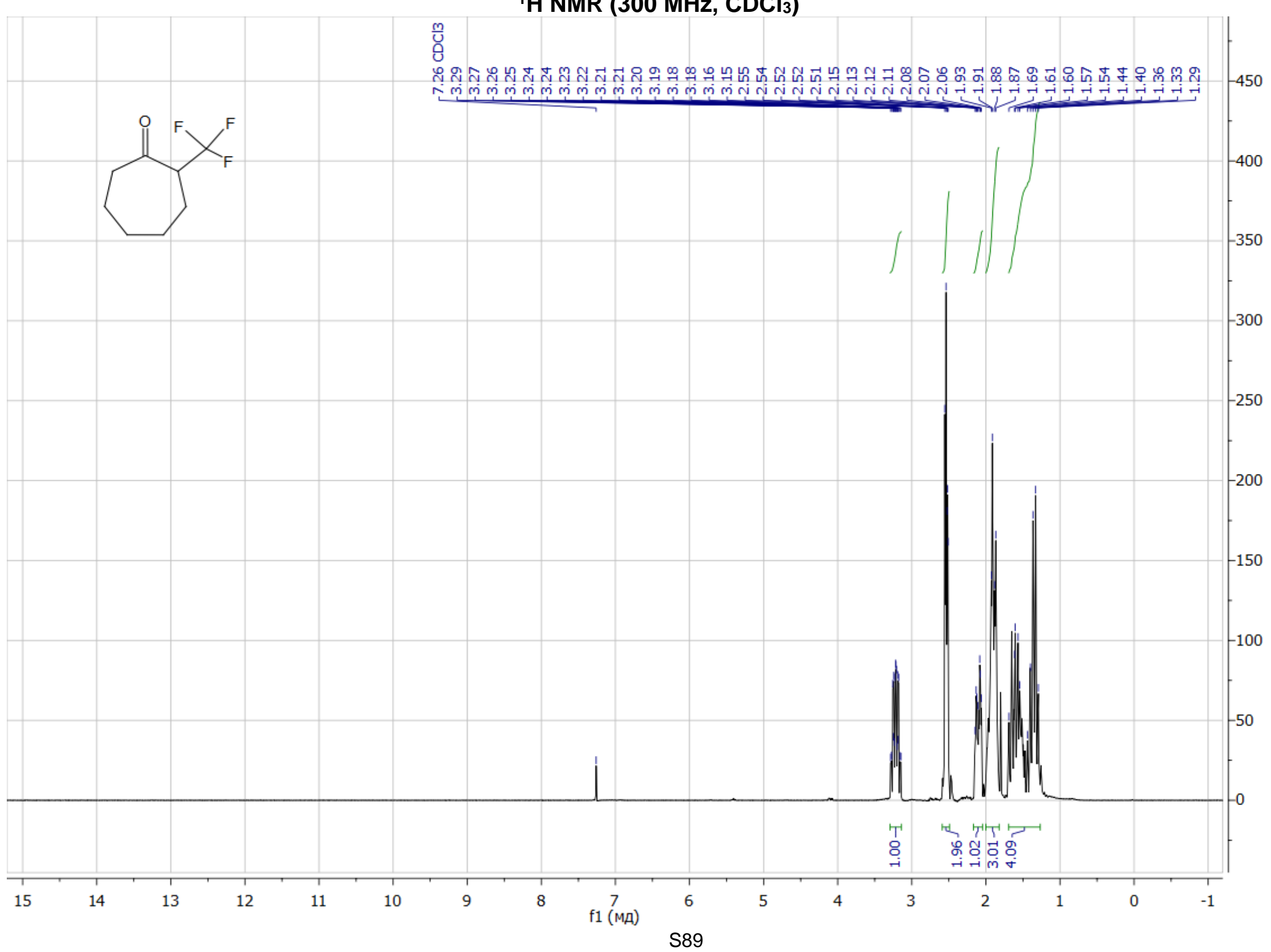


${ }^{13} \mathrm{C} \mathrm{NMR}\left(75 \mathrm{MHz}, \mathrm{CDCl}_{3}\right)$

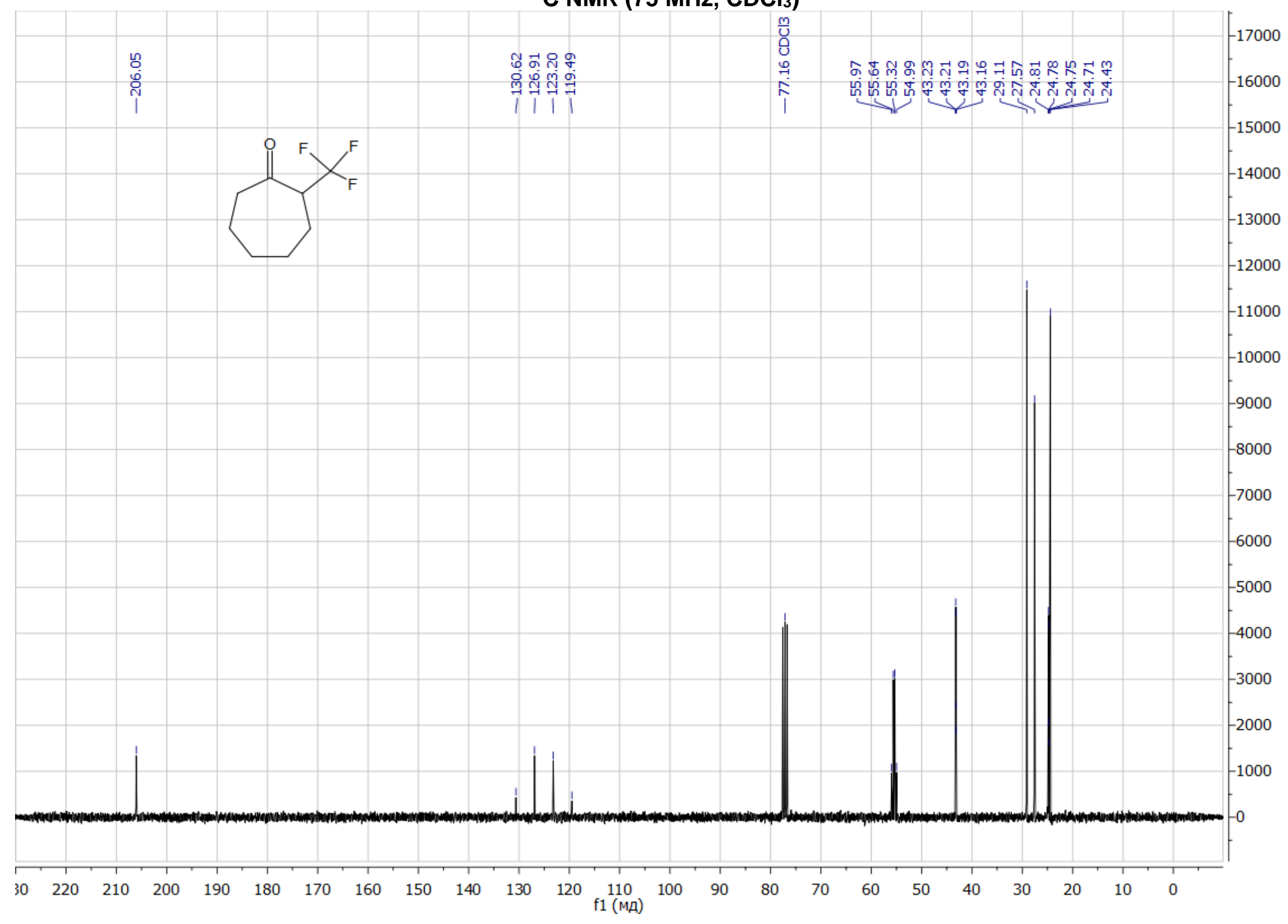


${ }^{19} \mathrm{~F}$ NMR (282 $\left.\mathrm{MHz}, \mathrm{CDCl}_{3}\right)$

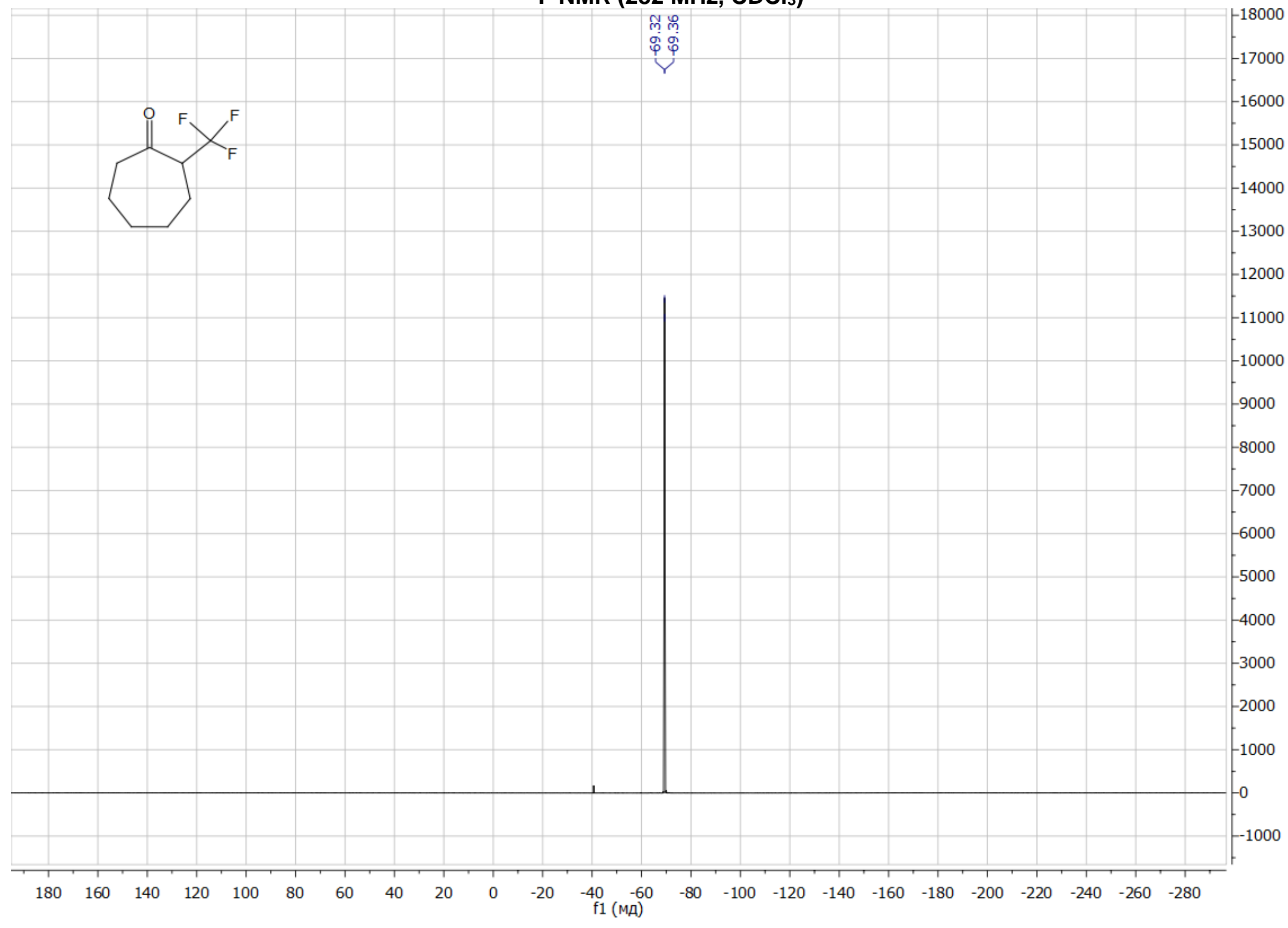


2-(Trifluoromethyl)cyclododecan-1-one, 20

${ }^{1} \mathrm{H}$ NMR (300 MHz, $\mathrm{CDCl}_{3}$ )

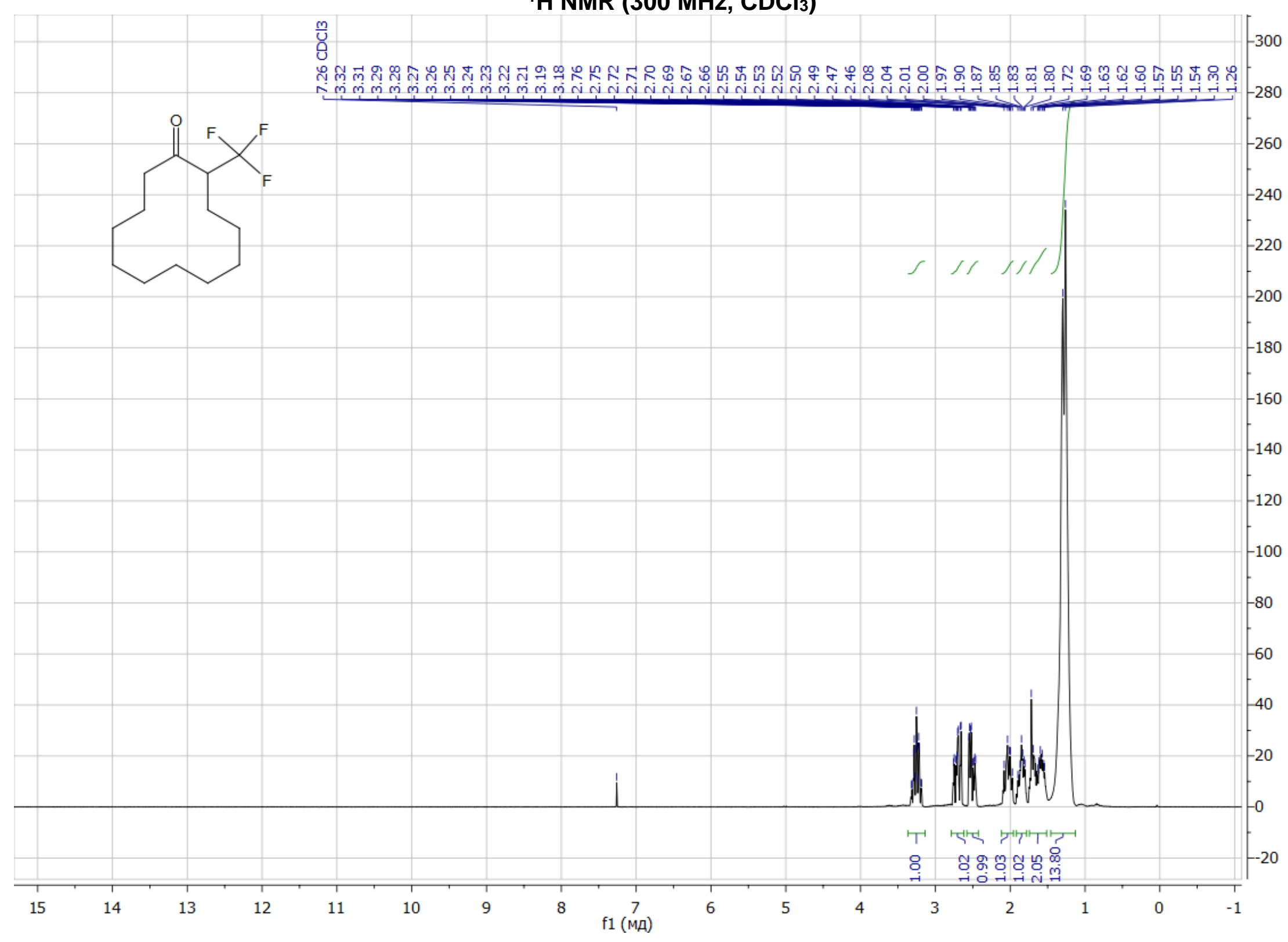


$\left.{ }^{13} \mathrm{C} \mathrm{NMR} \mathrm{(75} \mathrm{MHz,} \mathrm{CDCl}_{3}\right)$

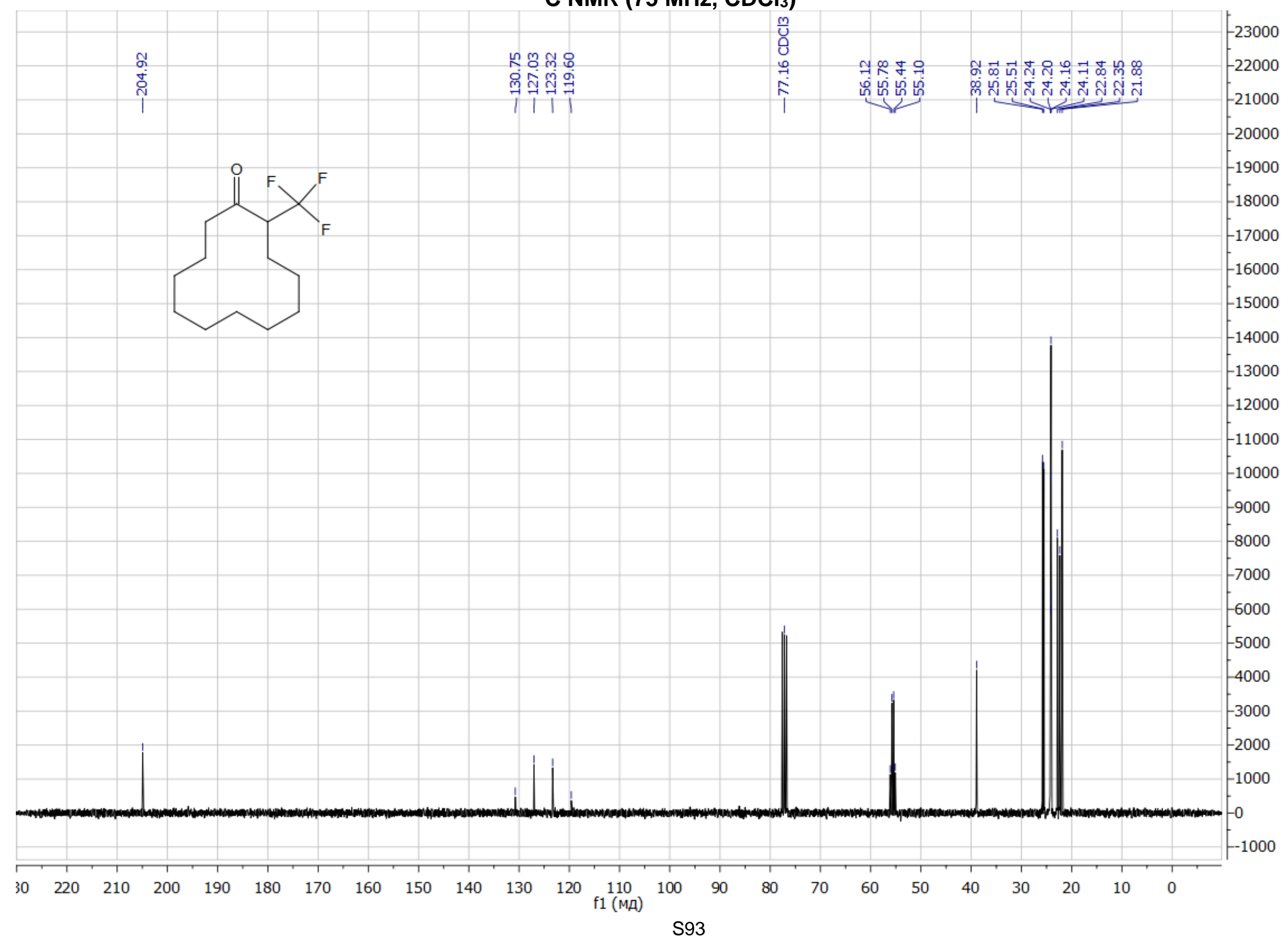


${ }^{19} \mathrm{~F}$ NMR (282 $\mathrm{MHz}, \mathrm{CDCl}_{3}$ )

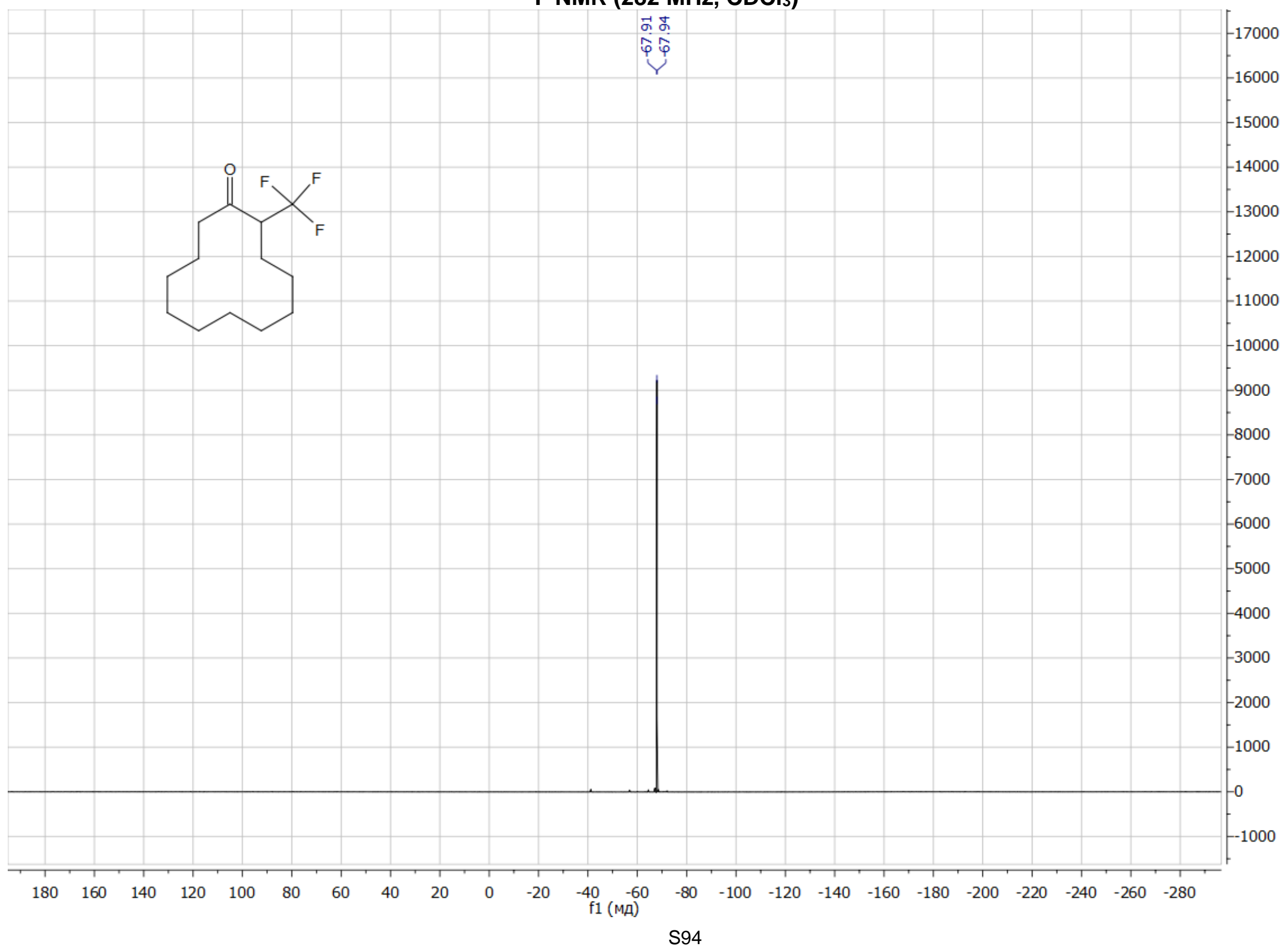


2-(Perfluorobutyl)-1-(4-methoxyphenyl)ethan-1-one, 3c ${ }^{1} \mathrm{H}$ NMR (300 MHz, CDCl 3 )

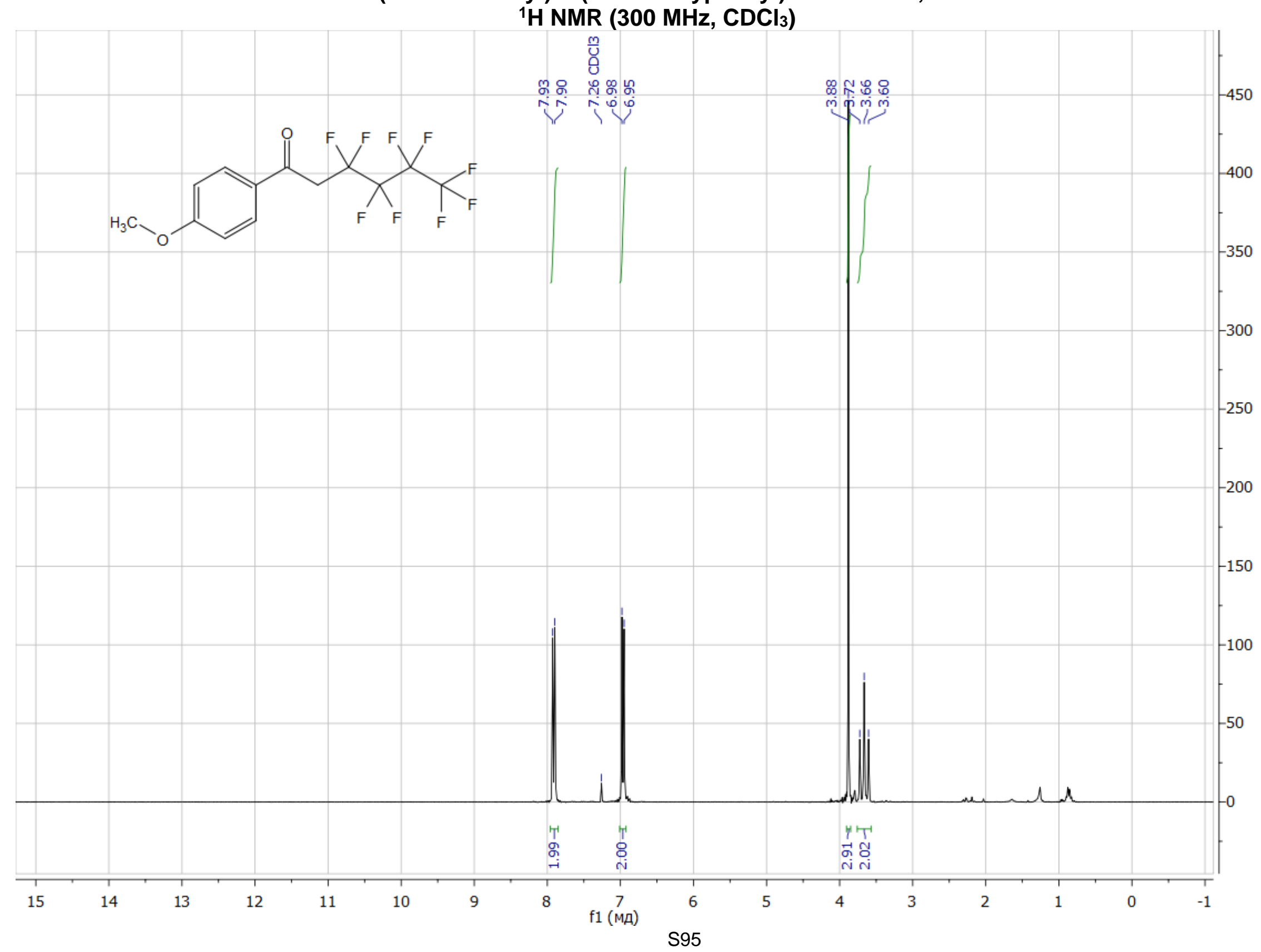


${ }^{13} \mathrm{C}$ NMR (75 MHz, $\left.\mathrm{CDCl}_{3}\right)$

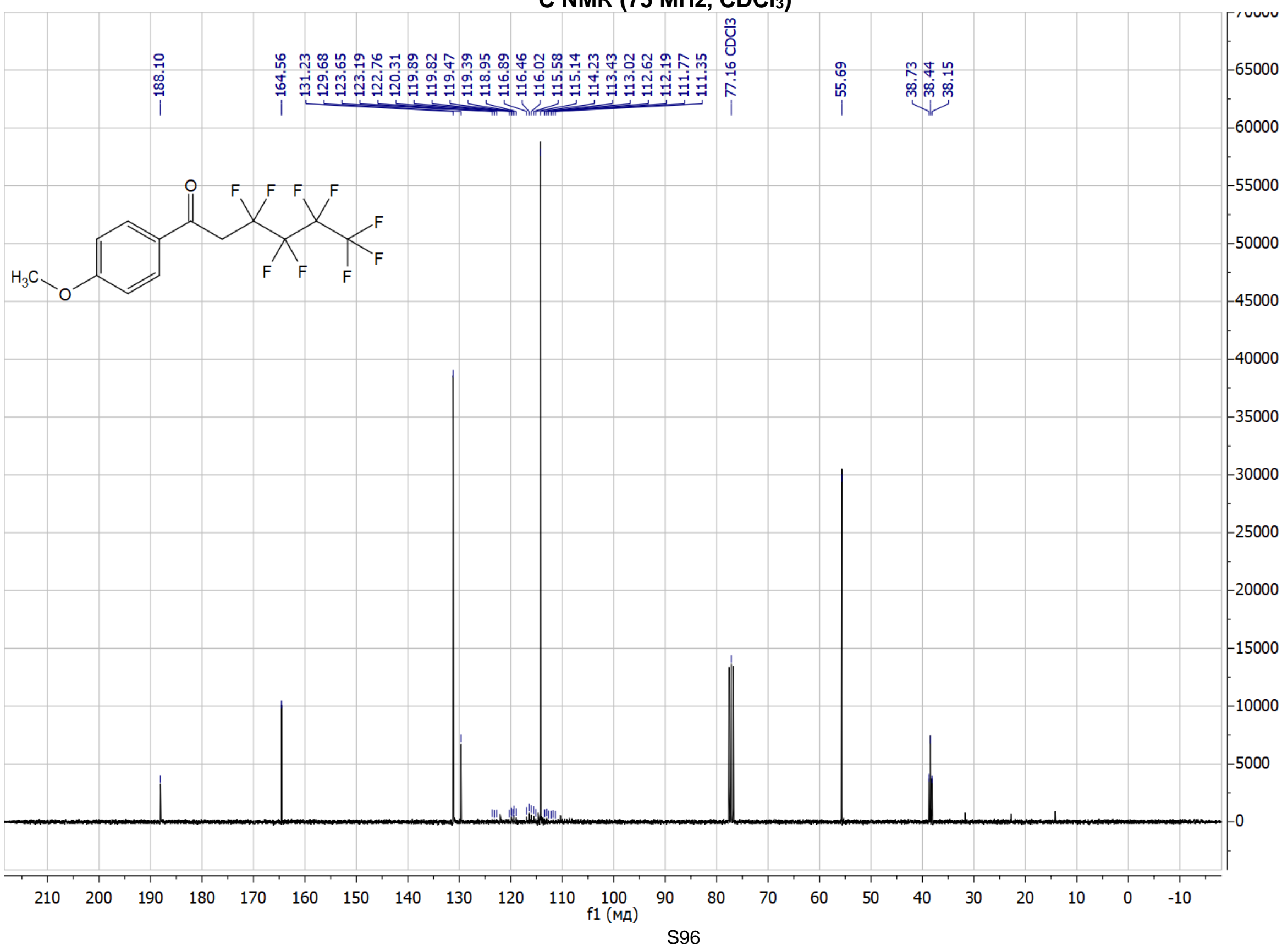


${ }^{19} \mathrm{~F}$ NMR (282 MHz, $\left.\mathrm{CDCl}_{3}\right)$

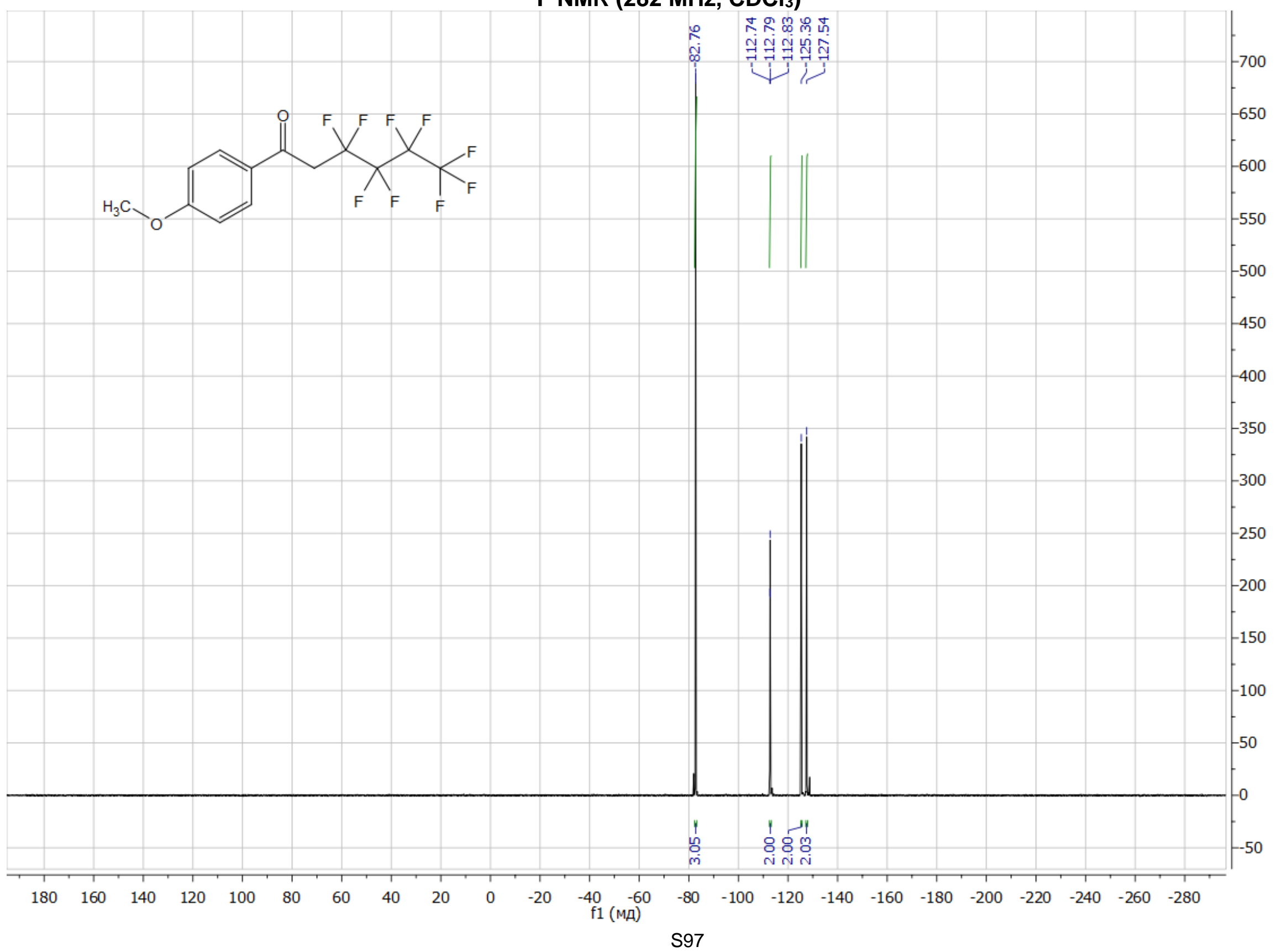


2-(Perfluorobutyl)-1-(4-chlorophenyl)ethan-1-one, 3d ${ }^{1} \mathrm{H}$ NMR (300 MHz, CDCl 3 )

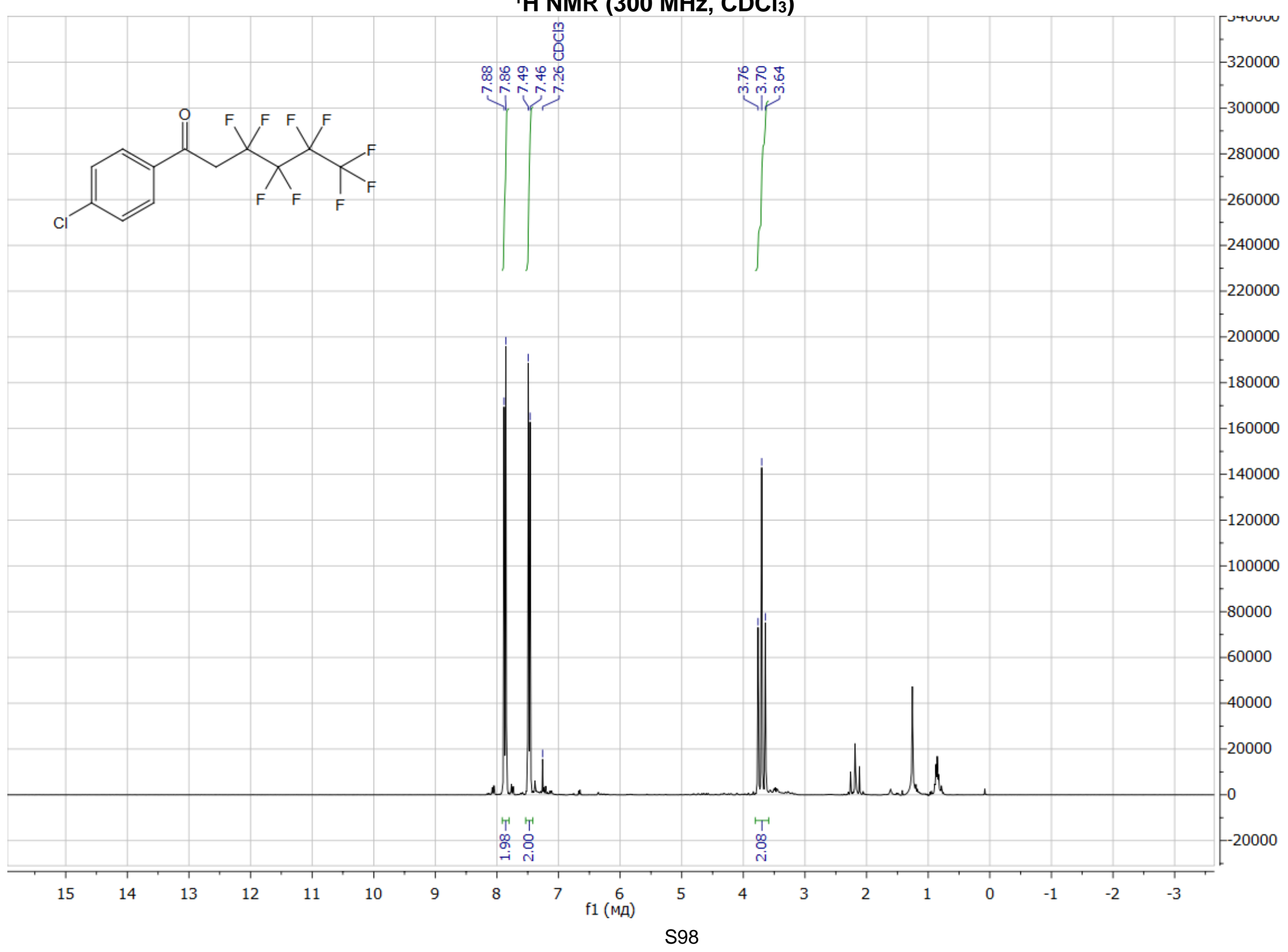


$\left.{ }^{13} \mathrm{C} \mathrm{NMR} \mathrm{(75} \mathrm{MHz,} \mathrm{CDCl}_{3}\right)$

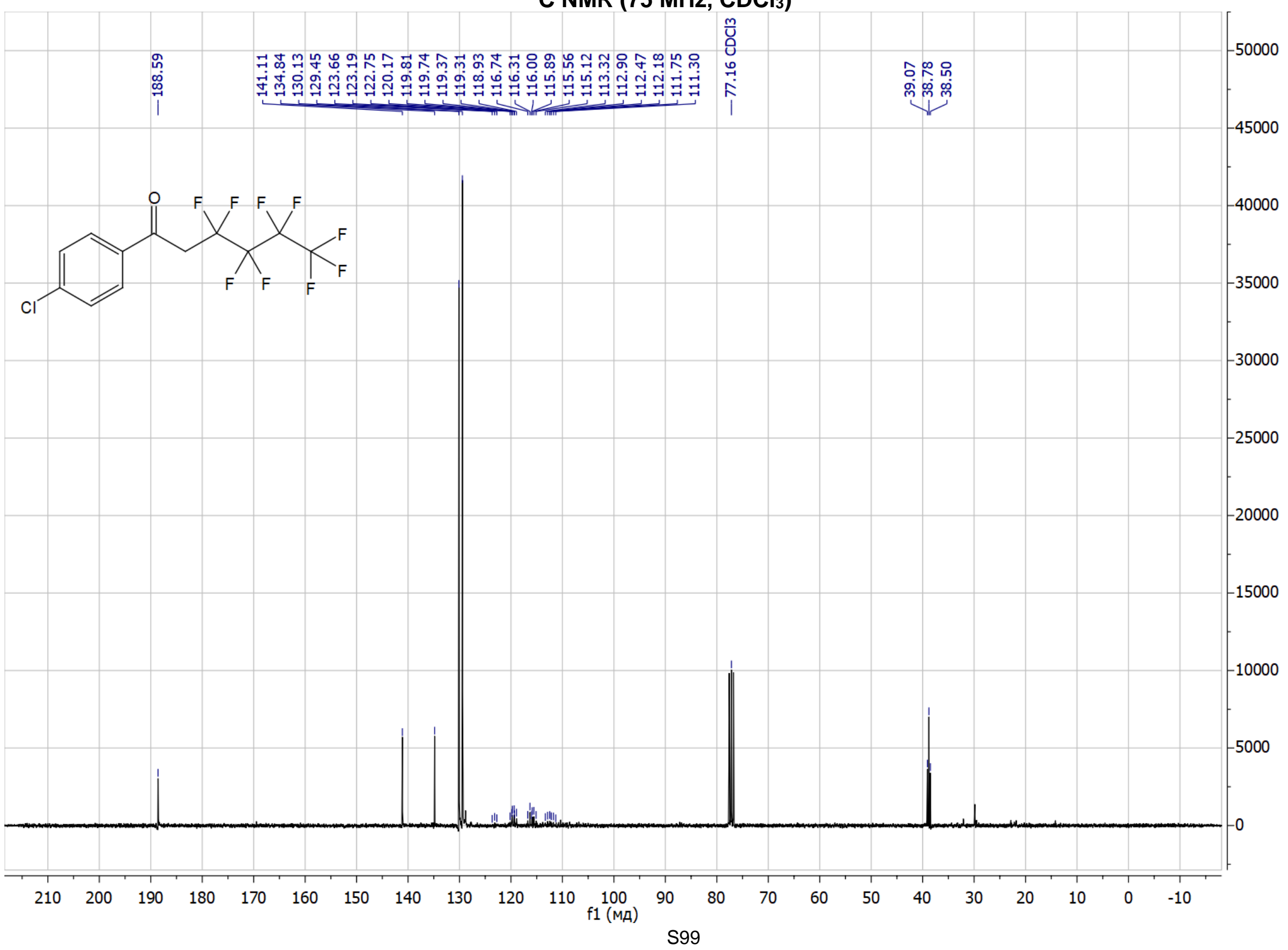


${ }^{19} \mathrm{~F}$ NMR (282 $\mathrm{MHz}, \mathrm{CDCl}_{3}$ )

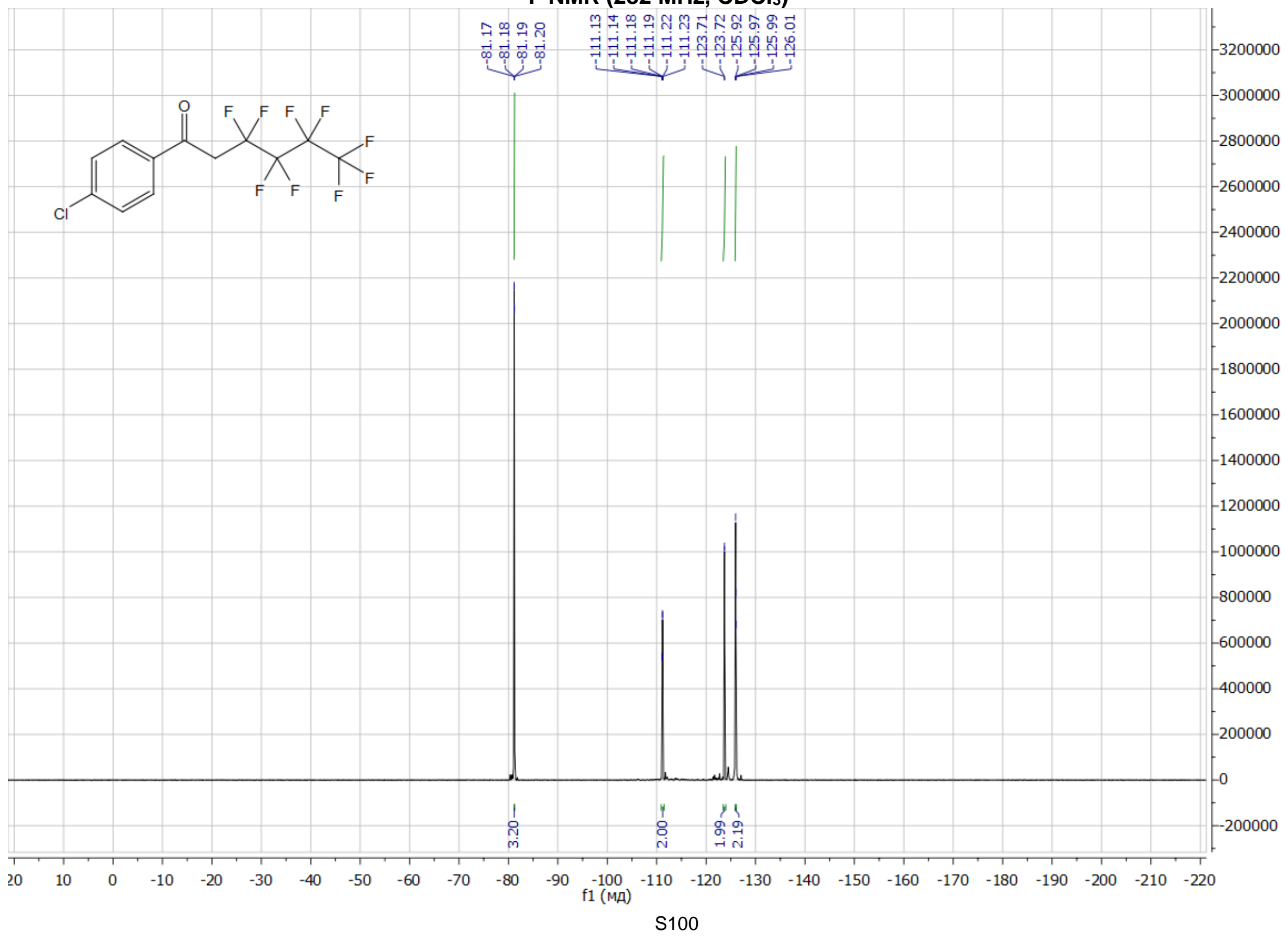


2-(Perfluorobutyl)-3,4-dihydronaphthalen-1(2H)-one, 3h

${ }^{1} \mathrm{H}$ NMR (300 MHz, $\mathrm{CDCl}_{3}$ )

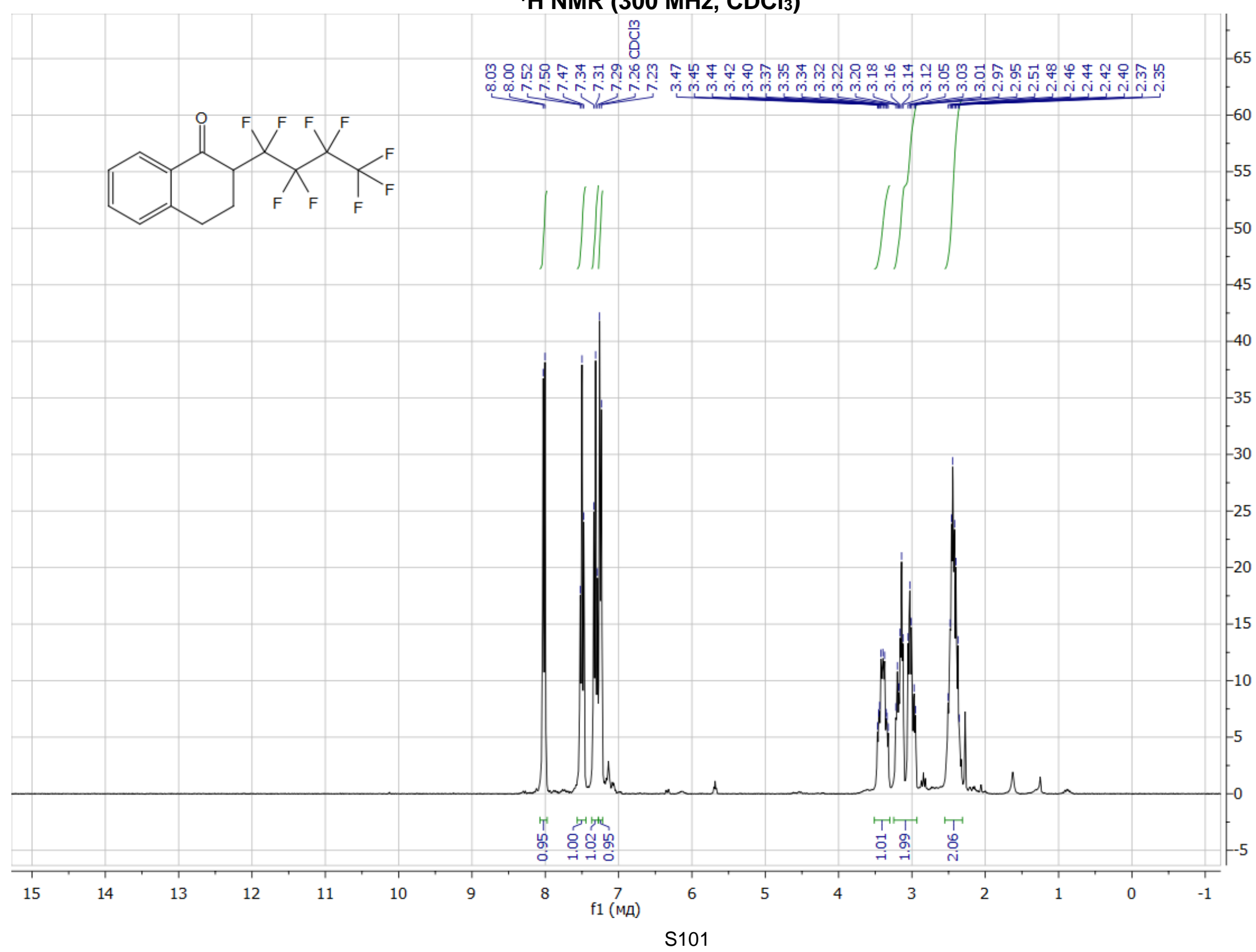


${ }^{13} \mathrm{C}$ NMR (75 MHz, $\left.\mathrm{CDCl}_{3}\right)$

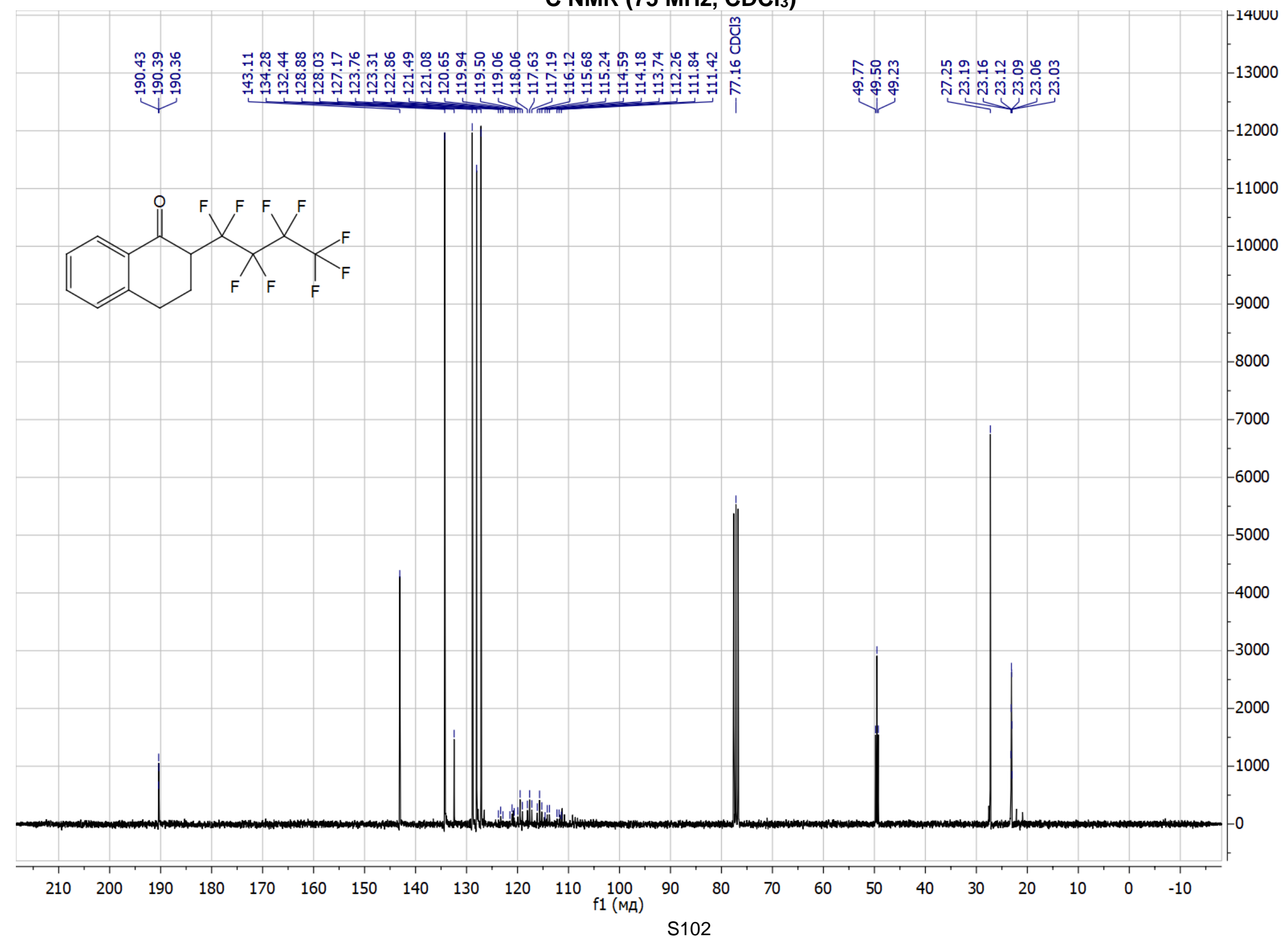


${ }^{19} \mathrm{~F}$ NMR (282 $\mathrm{MHz}, \mathrm{CDCl}_{3}$ )

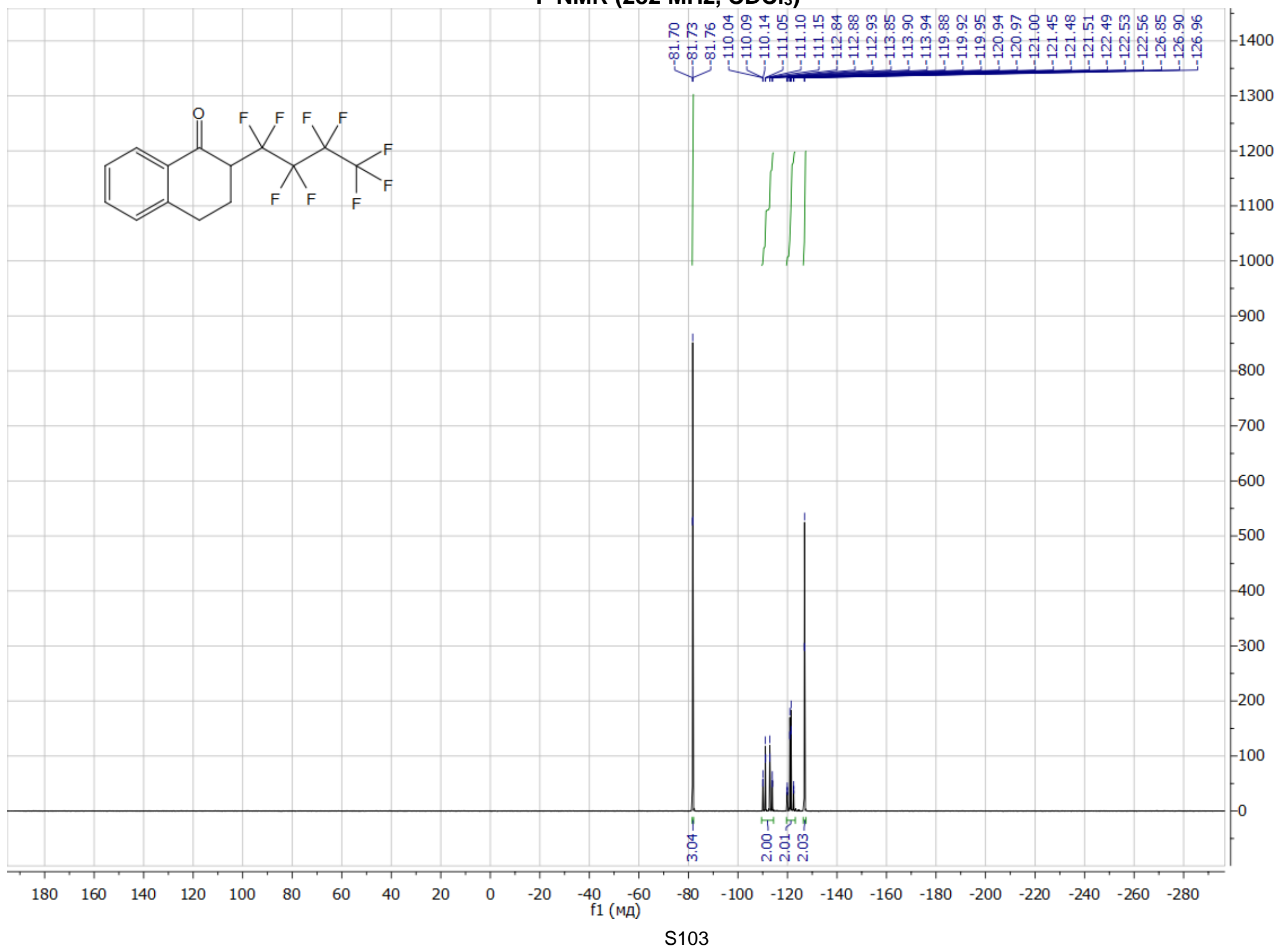


2-(Perfluorobutyl)-1-(phenyl)propan-1-one, 3i

${ }^{1} \mathrm{H}$ NMR (300 MHz, CDCl 3 )

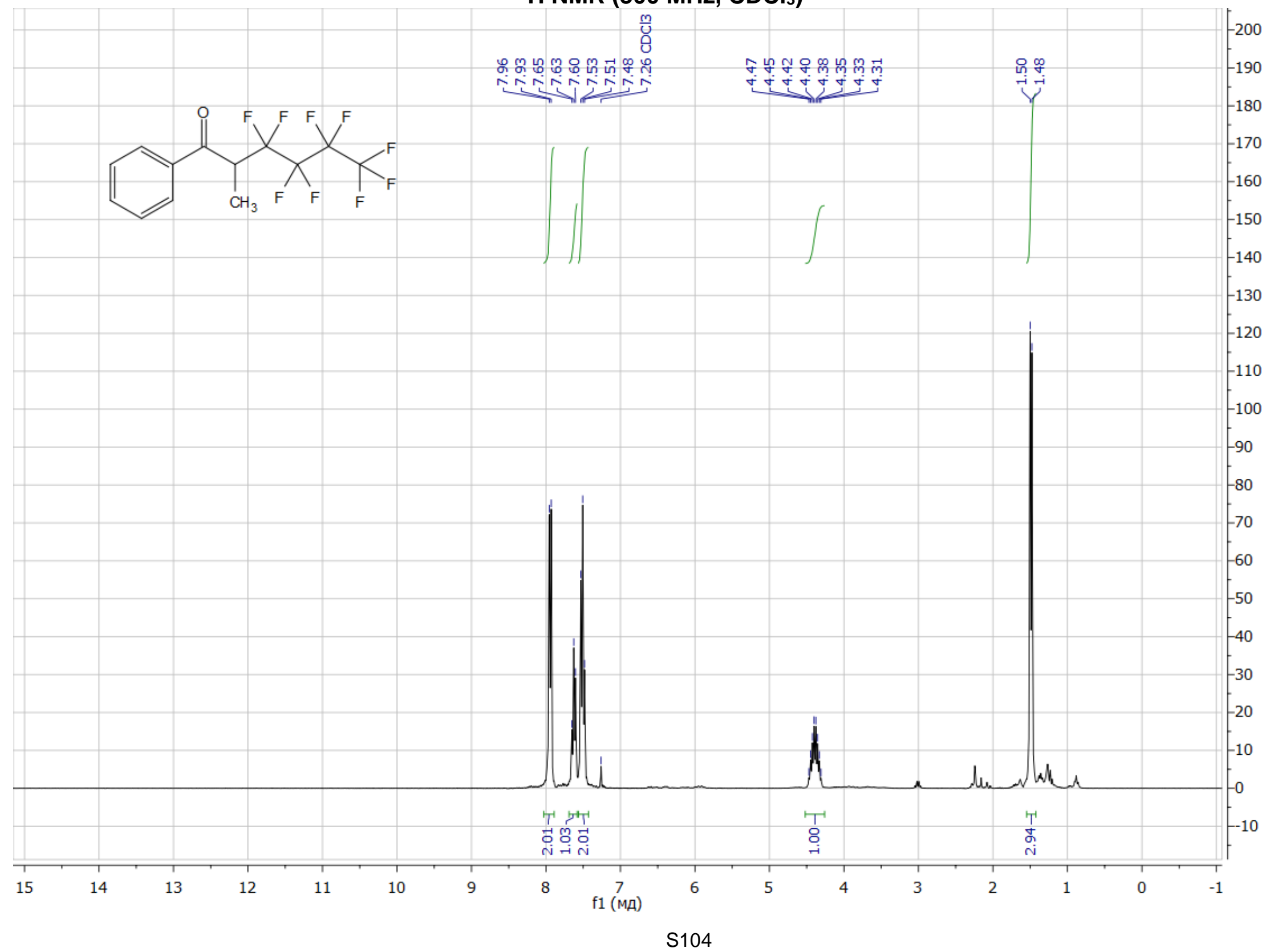


$\left.{ }^{13} \mathrm{C} \mathrm{NMR} \mathrm{(75} \mathrm{MHz,} \mathrm{CDCl}_{3}\right)$

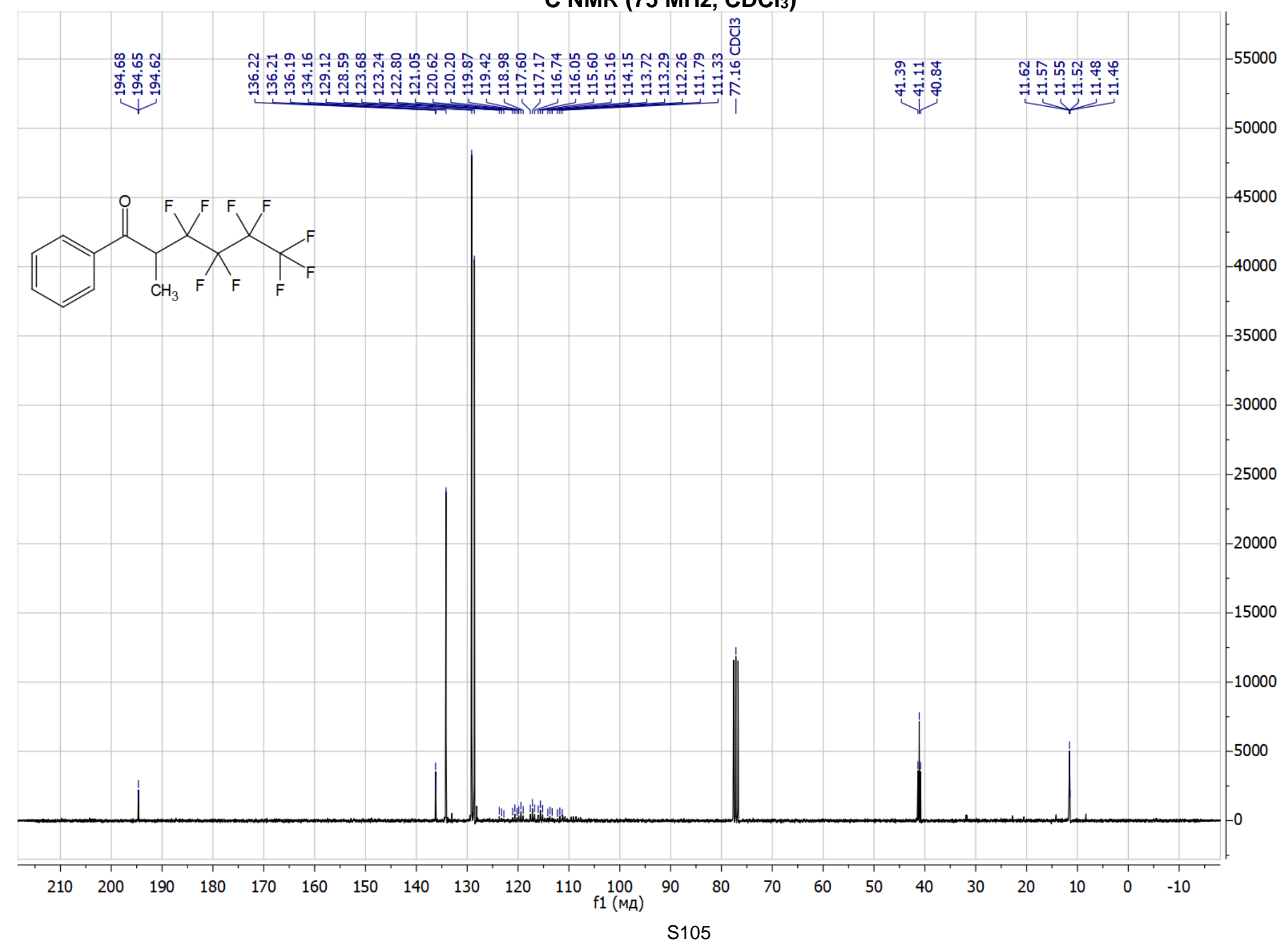


${ }^{19} \mathrm{~F}$ NMR (282 MHz, $\left.\mathrm{CDCl}_{3}\right)$

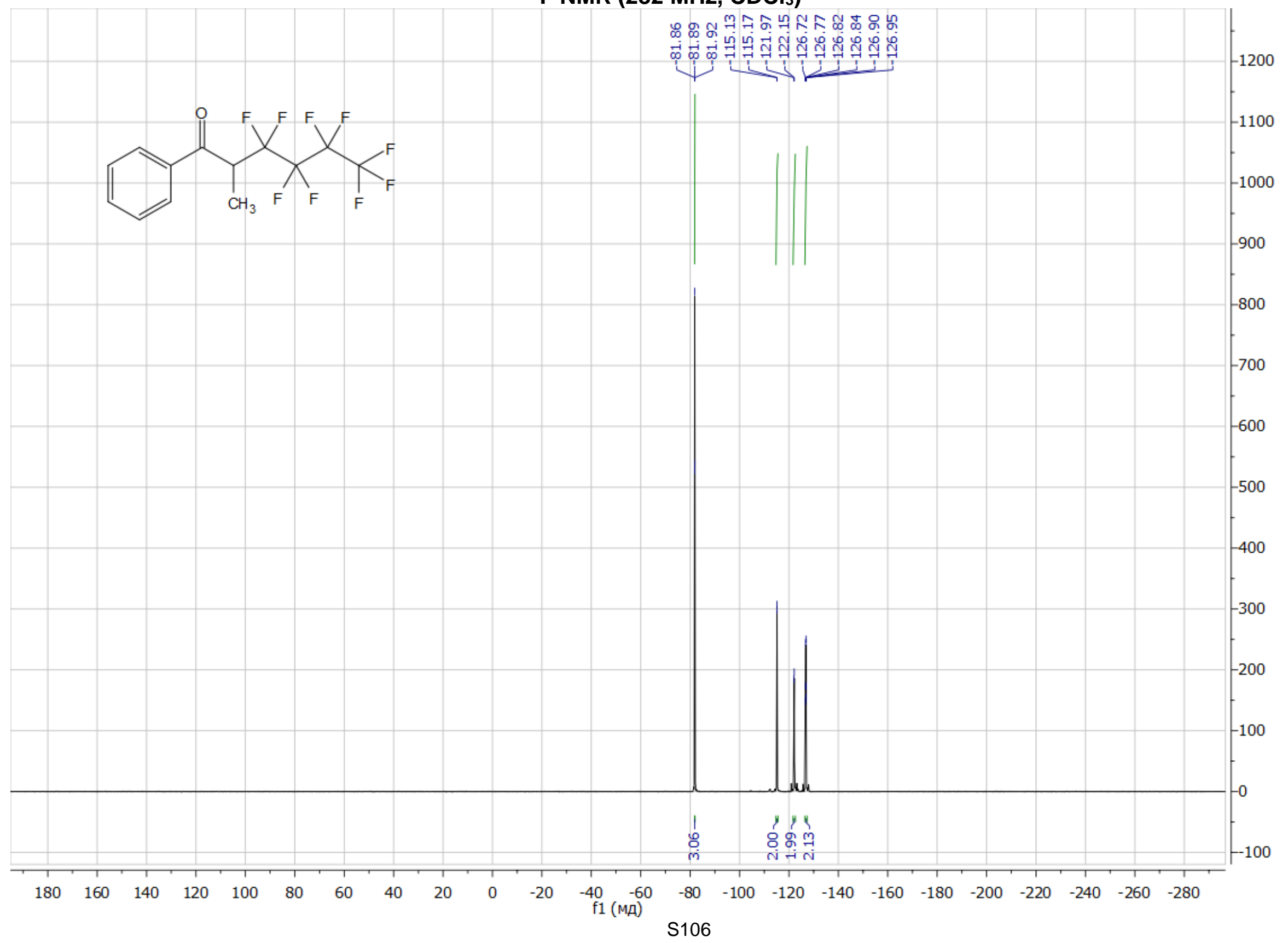


2-Acetoxyacetophenone, 4a

${ }^{1} \mathrm{H}$ NMR (300 MHz, CDCl ${ }_{3}$ )

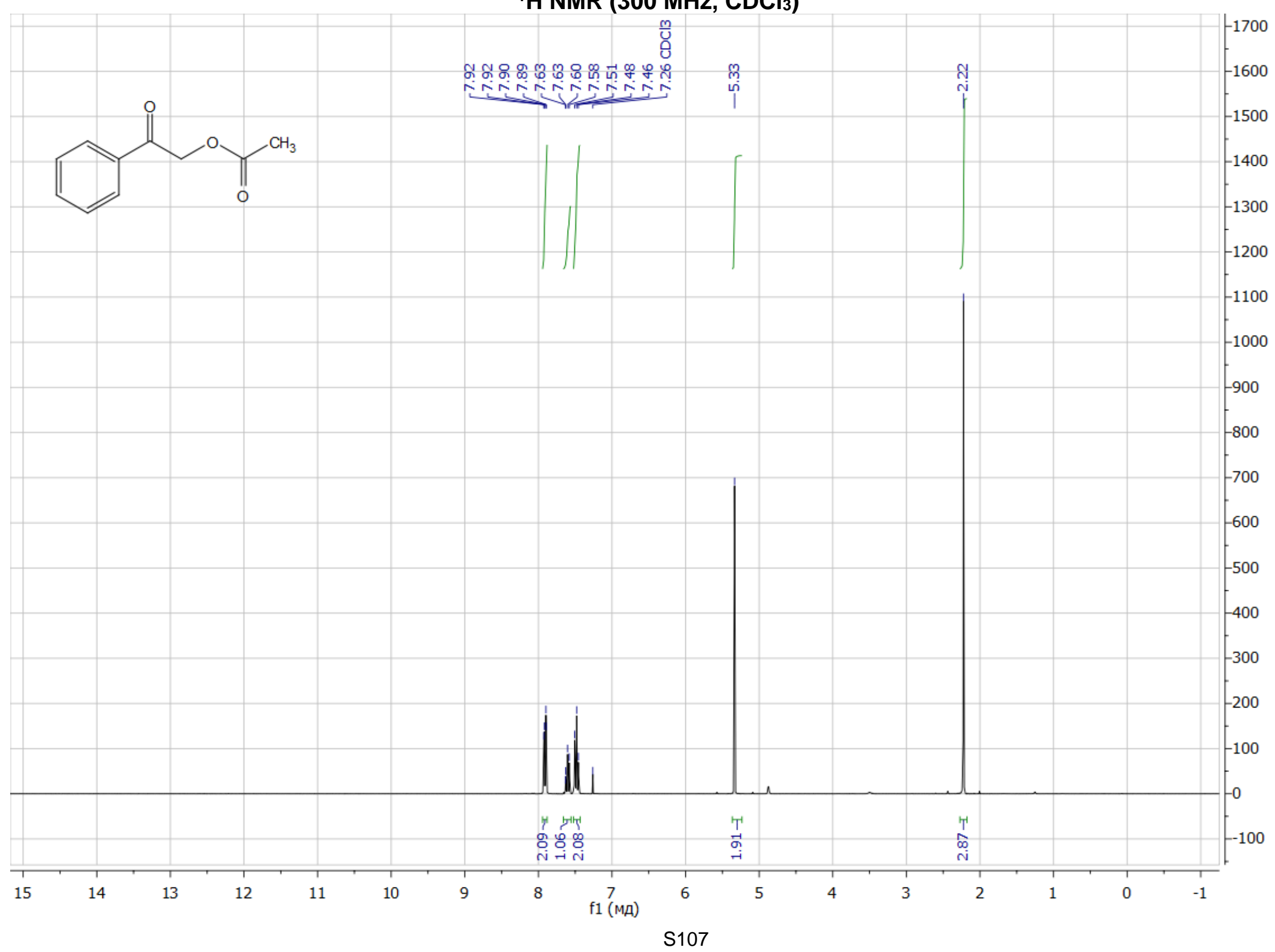


$\left.{ }^{13} \mathrm{C} \mathrm{NMR} \mathrm{(75} \mathrm{MHz,} \mathrm{CDCl}_{3}\right)$

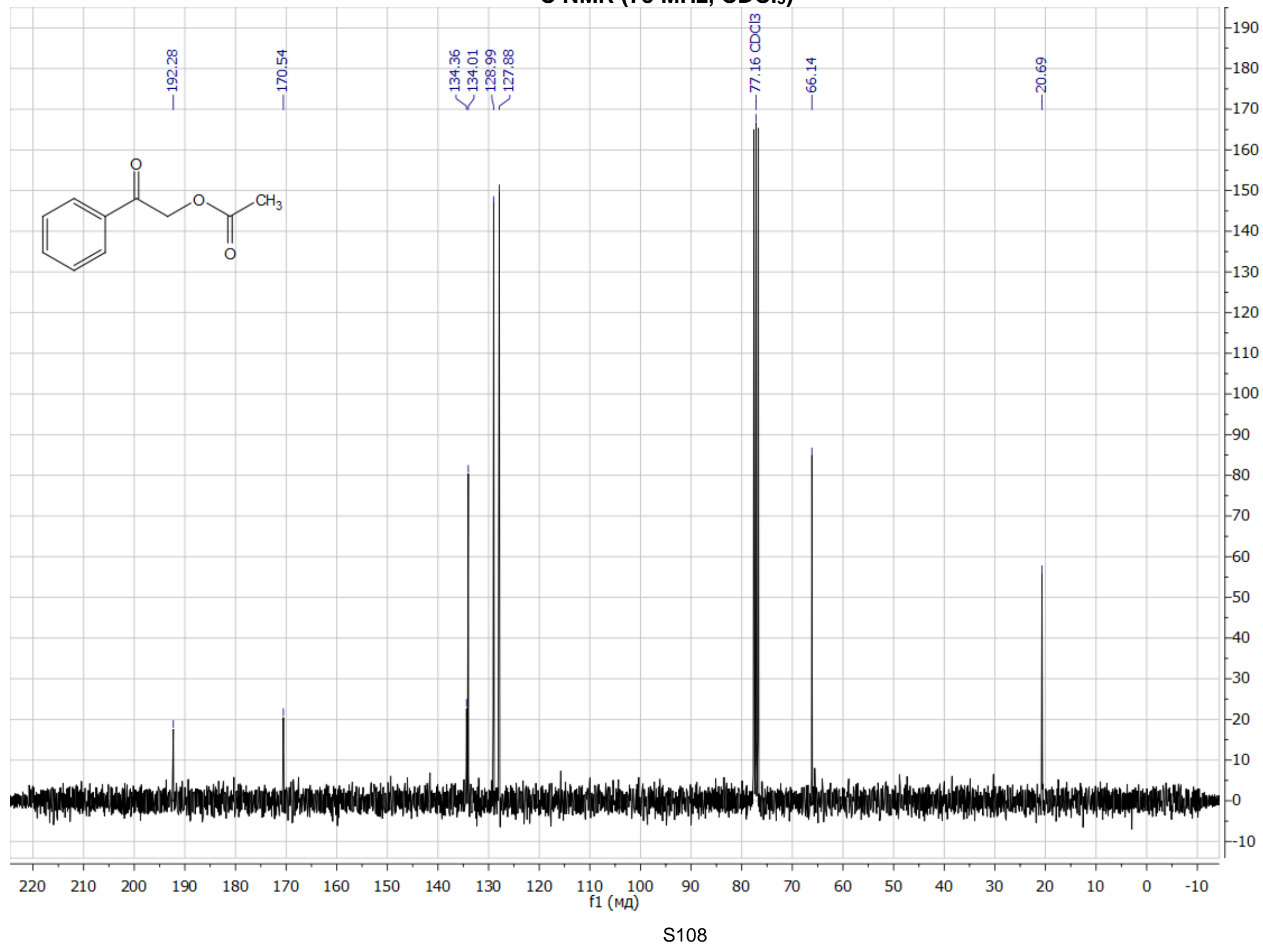


2-Hydroxyacetophenone, 5a

${ }^{1} \mathrm{H}$ NMR (300 MHz, CDCl 3 )

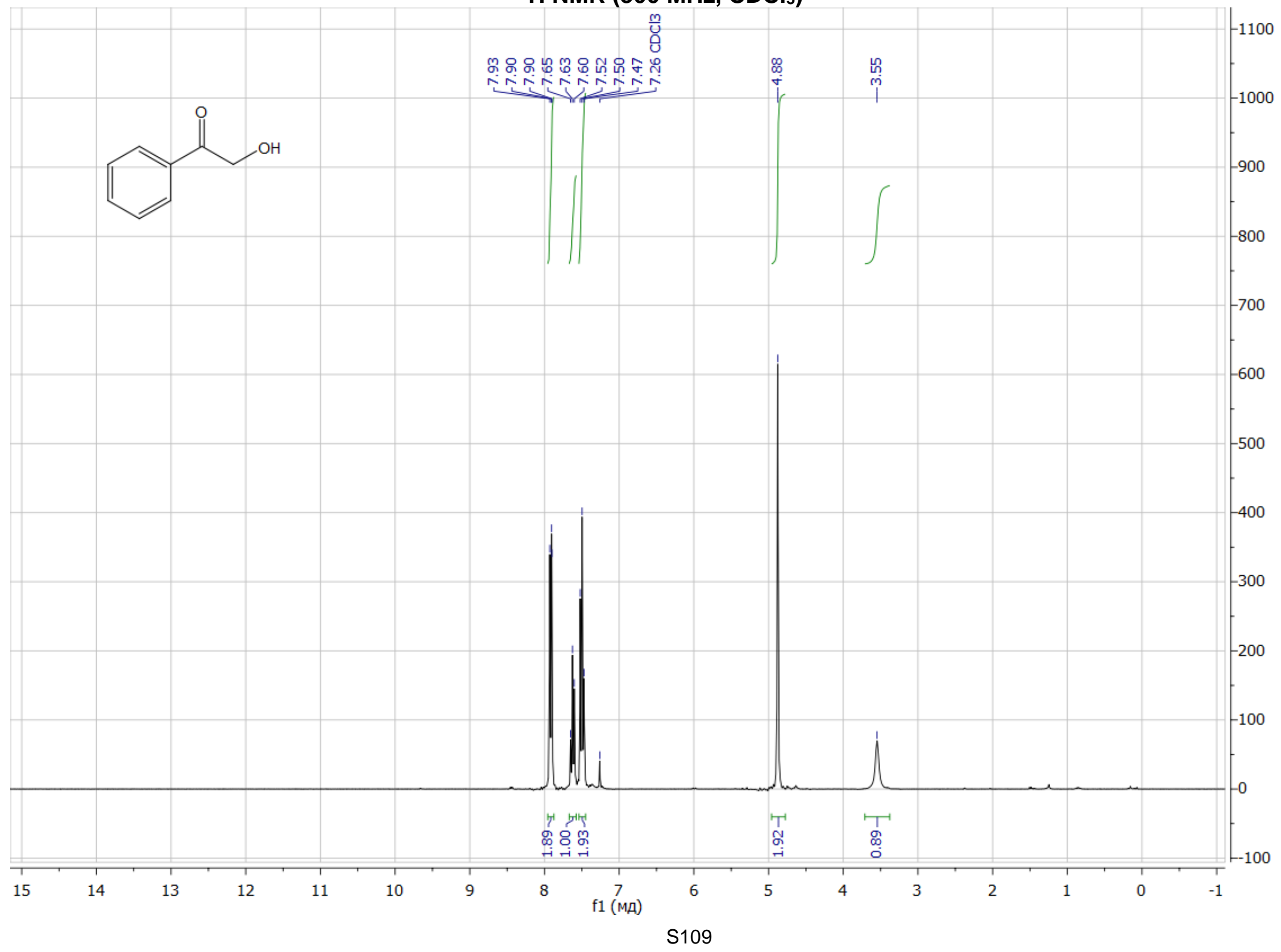


${ }^{13} \mathrm{C}$ NMR (75 MHz, $\left.\mathrm{CDCl}_{3}\right)$

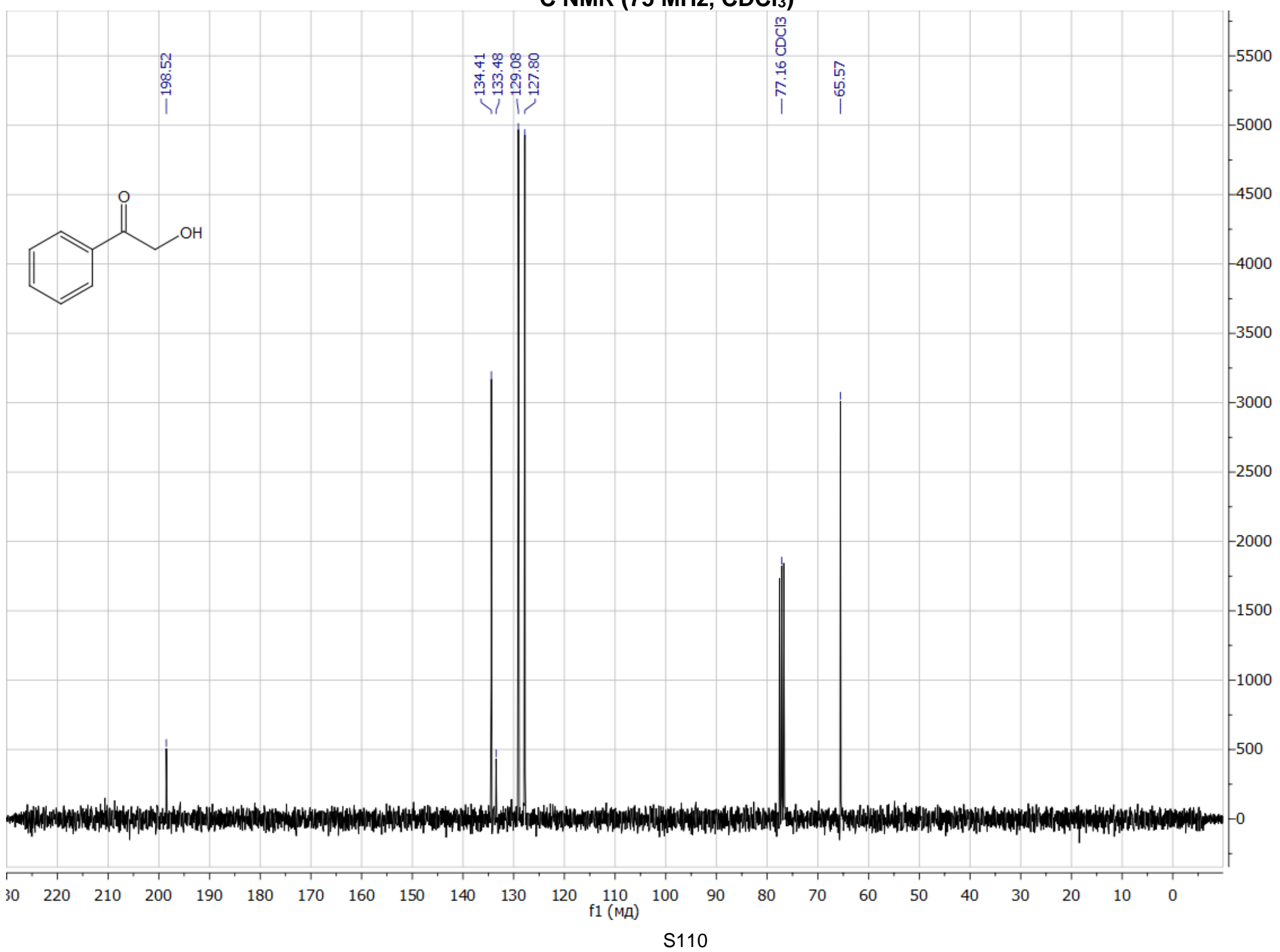


HRMS spectra of obtained fluorinated ketones

2-(Trifluoromethyl)cyclododecan-1-one, 20

\section{Display Report}

\section{Analysis Info}

Analysis Name

Method

Sample Name

Comment
D:IData|Kolotyrkinal2020।Vil\0727010.d

tune_50-1600.m

/TERN BB-2335-2

$\mathrm{C} 13 \mathrm{H} 21 \mathrm{~F} 3 \mathrm{O} \mathrm{mH} 251.1317 \mathrm{clb}$ added $\mathrm{CH} 3 \mathrm{CN}$

Acquisition Date 27.07.2020 15:57:04

Operator BDAL@DE

Instrument / Ser\# micrOTOF 10248

\begin{tabular}{llllll}
\hline Acquisition Parameter & & & & \\
Source Type & ESI & Ion Polarity & Positive & Set Nebulizer & 1.0 Bar \\
Focus & Not active & & & Set Dry Heater & $2000^{\circ} \mathrm{C}$ \\
Scan Begin & $50 \mathrm{~m} / \mathrm{z}$ & Set Capillary & $4500 \mathrm{~V}$ & Set Dry Gas & $4.0 \mathrm{~V} / \mathrm{min}$ \\
Scan End & $1600 \mathrm{~m} / \mathrm{z}$ & Set End Plate Offset & $-500 \mathrm{~V}$ & Set Divert Valve & Waste \\
\hline
\end{tabular}

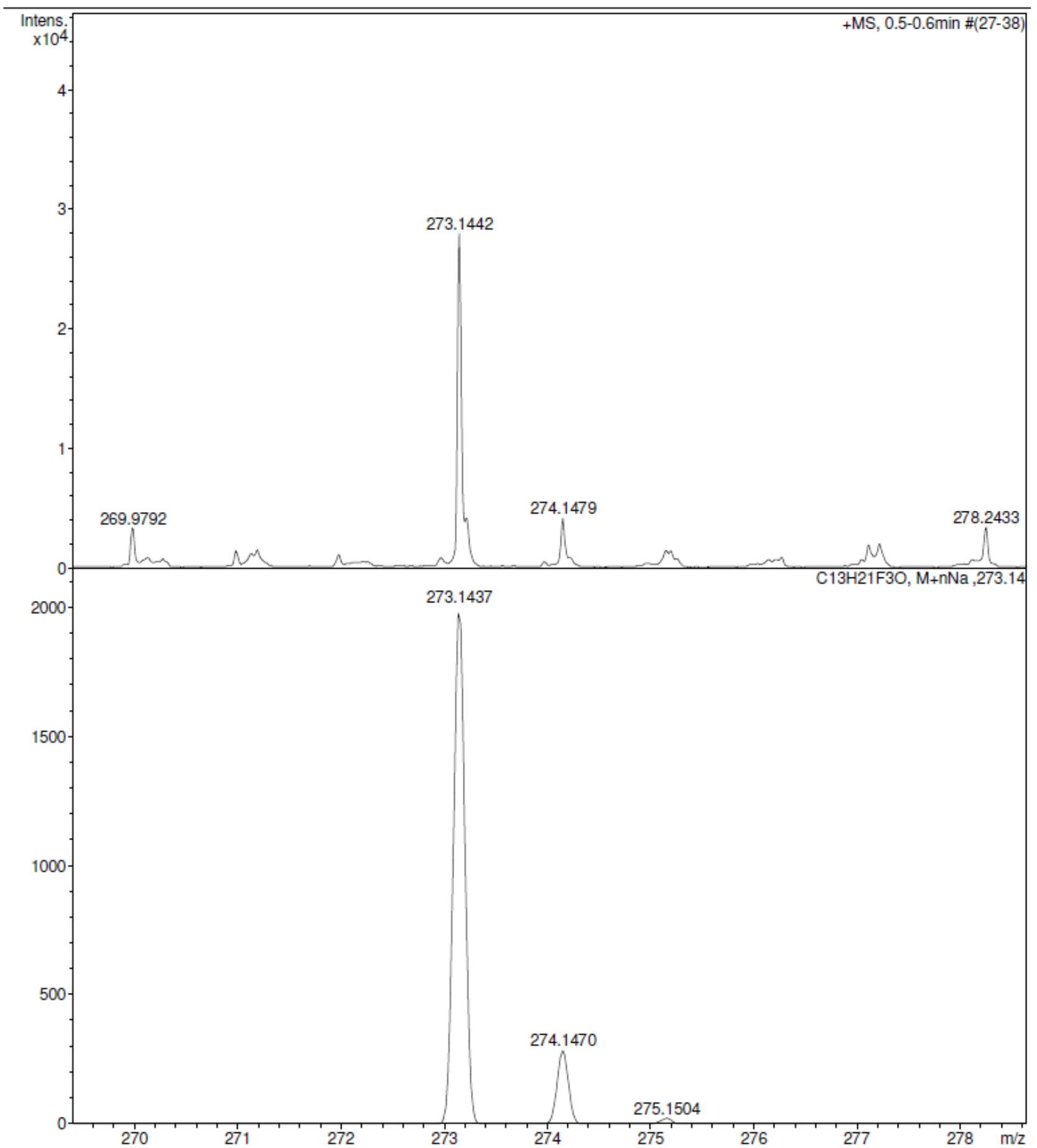




\section{2-(Perfluorobutyl)-1-(4-methoxyphenyl)ethan-1-one, 3c}

Display Report

Analysis Info

Analysis Name

Method

Sample Name

Comment
Acquisition Date 24.11.2020 16:33:45

D:Data|Kolotyrkinal2020|Vil\1124027.d tune 50-1600.m

Operator

BDAL@DE

Instrument / Ser\# micrOTOF

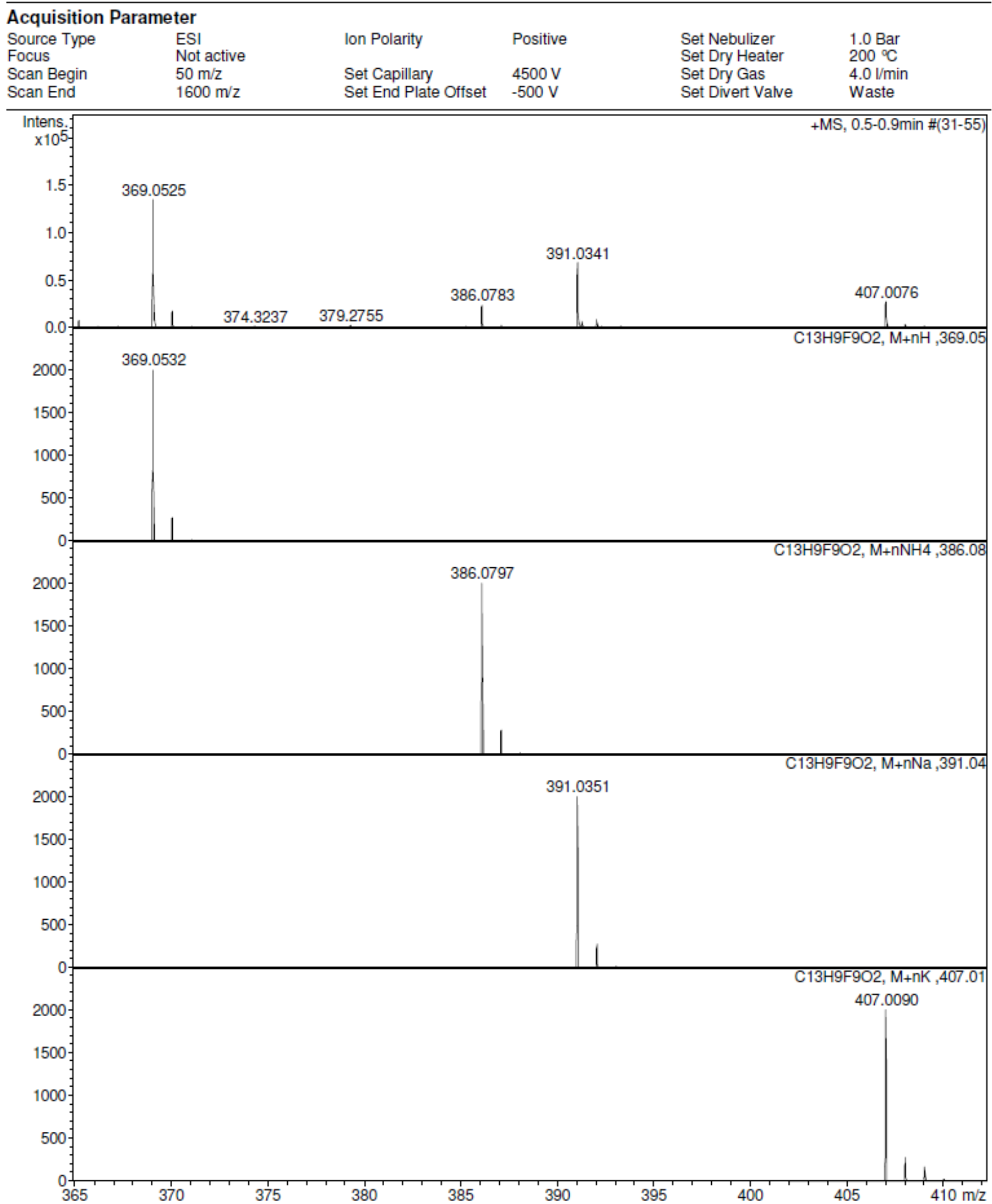


2-(Perfluorobutyl)-1-(4-chlorophenyl)ethan-1-one, 3d

Display Report

Analysis Info

Analysis Name Method

Sample Name

Comment
Acquisition Date

24.11.2020 16:39:16

Operator BDAL@DE

Instrument / Ser\# micrOTOF

10248

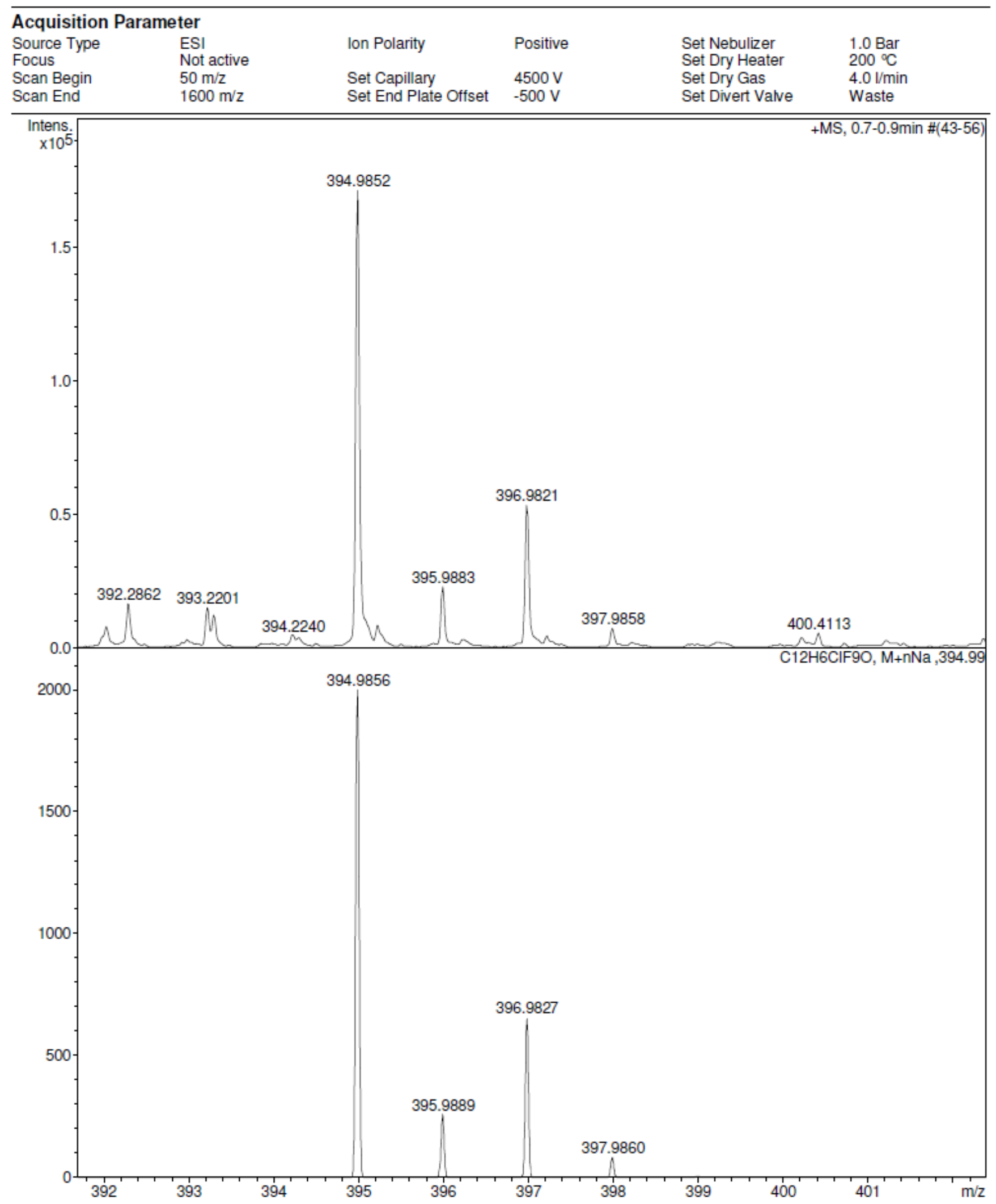


2-(Perfluorobutyl)-3,4-dihydronaphthalen-1(2H)-one, 3h

Display Report

Analysis Info

Analysis Name

Method

Sample Name

Comment
Acquisition Date $\quad 25.11 .202016: 56: 07$

D:DatalKolotyrkinal2020lVil1125030.d

tune 50-1600.m

Operator BDAL@DE

Instrument / Ser\# micrOTOF 10248

\begin{tabular}{llllll}
\hline \multicolumn{2}{l}{ Acquisition Parameter } & & & & \\
Source Type & ESI & Ion Polarity & Positive & Set Nabulizer & 1.0 Bar \\
Focus & Not active & & & Set Dry Heater & $2000^{\circ} \mathrm{C}$ \\
Scan Begin & $50 \mathrm{~m} / \mathrm{z}$ & Set Capillary & $4500 \mathrm{~V}$ & Set Dry Gas & $4.0^{\circ}$ Vmin \\
Scan End & $1600 \mathrm{~m} / \mathrm{z}$ & Set End Plate Offset & $-500 \mathrm{~V}$ & Set DivertValve & Waste \\
\hline
\end{tabular}

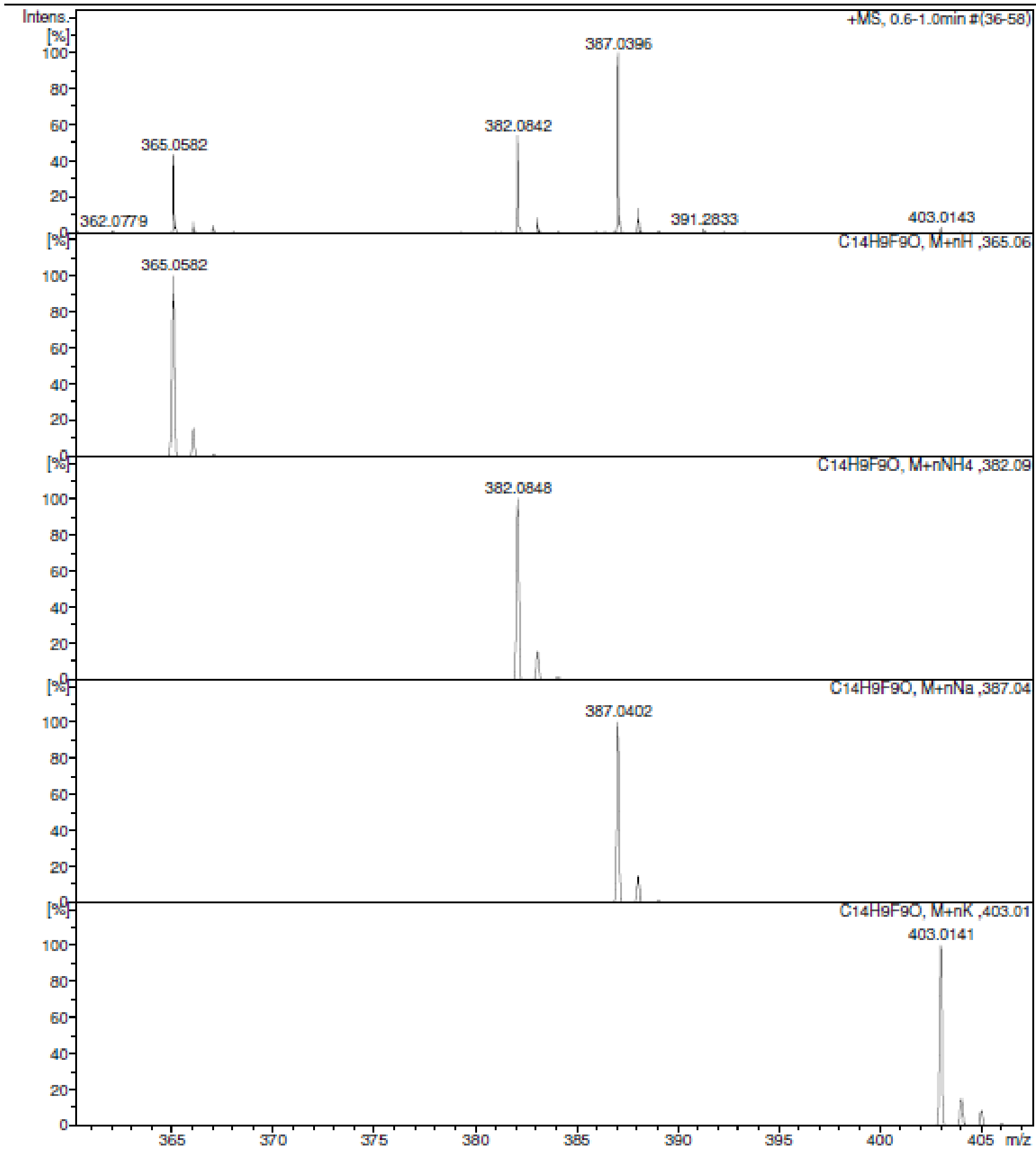


2-(Perfluorobutyl)-1-(phenyl)propan-1-one, 3i

Display Report

Analysis Info

Analysis Name

Method

Sample Name

Comment
Acquisition Date 27.11.2020 16:22:09

Operator BDAL@DE

Instrument / Ser\# micrOTOF 10248
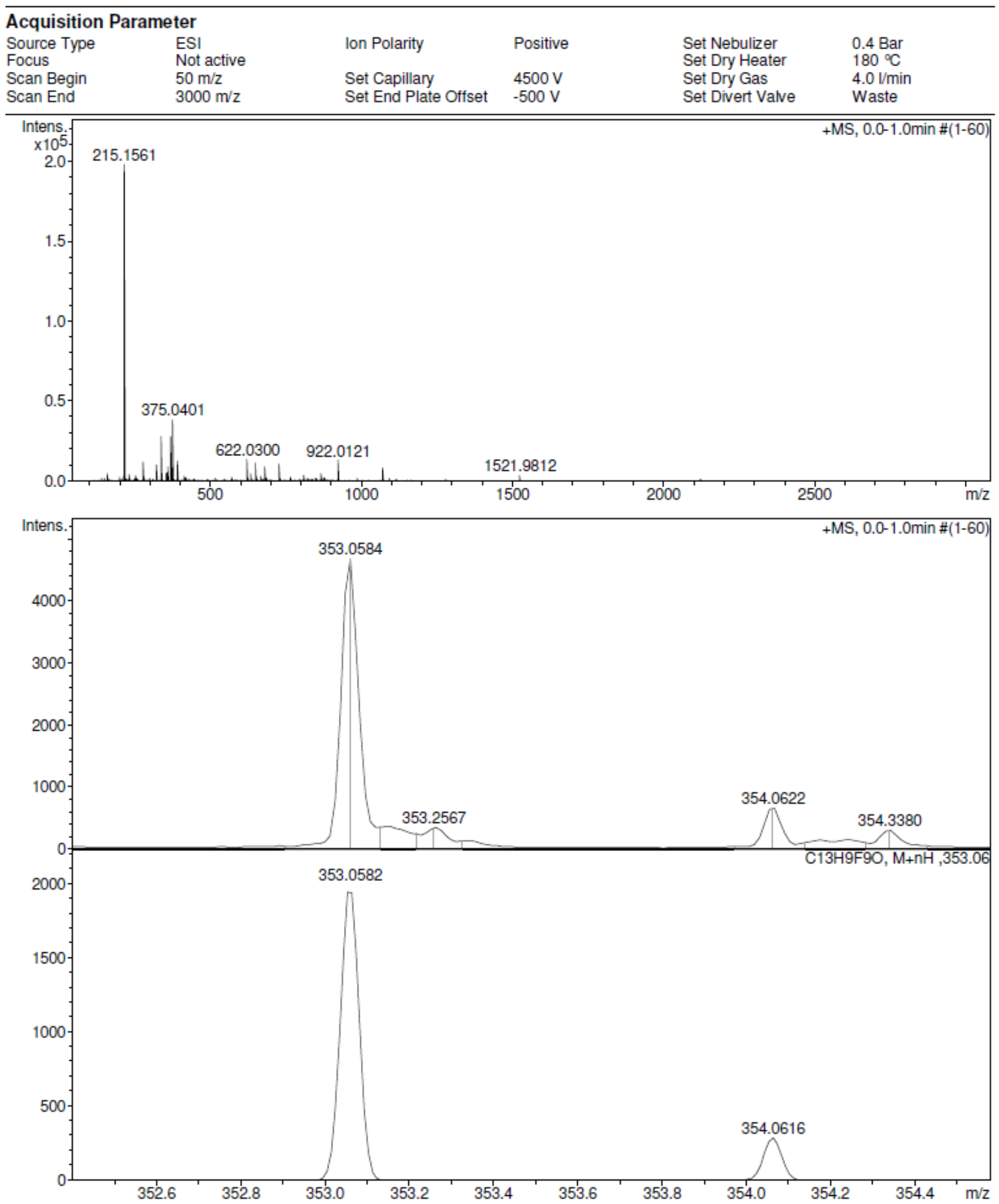